Portland State University

PDXScholar

Summer 7-5-2017

\title{
Socioeconomic Determinants of Health Disparities by Race and Ethnicity: the Mediating Role of Social, Psychological and Behavioral Factors
}

Amanuel Zimam Melekin

Portland State University

Follow this and additional works at: https://pdxscholar.library.pdx.edu/open_access_etds

Part of the Health Services Research Commons, Inequality and Stratification Commons, and the Medicine and Health Commons

Let us know how access to this document benefits you.

\section{Recommended Citation}

Melekin, Amanuel Zimam, "Socioeconomic Determinants of Health Disparities by Race and Ethnicity: the Mediating Role of Social, Psychological and Behavioral Factors" (2017). Dissertations and Theses. Paper 3765.

https://doi.org/10.15760/etd.5649

This Dissertation is brought to you for free and open access. It has been accepted for inclusion in Dissertations and Theses by an authorized administrator of PDXScholar. Please contact us if we can make this document more accessible: pdxscholar@pdx.edu. 
Socioeconomic Determinants of Health Disparities by Race and Ethnicity: The Mediating Role of Social, Psychological and Behavioral Factors

by

Amanuel Zimam Melekin

A dissertation submitted in partial fulfillment of the requirements for the degree of

Doctor of Philosophy

in

Systems Science

\author{
Dissertation Committee: \\ Martin Zwick, Chair \\ Jason Newsom \\ Neal Wallace \\ Kerth O'Brien \\ Lindsey Wilkinson
}

Portland State University

2017 
CC 2017 Amanuel Zimam Melekin 


\begin{abstract}
Socioeconomic status (SES) is inversely related to health status. Disparities in health status among races and ethnic groups are partly attributable to differences in SES, but the indirect pathways by which SES may influence health status are not widely studied.
\end{abstract}

Using the Health and Retirement Study (HRS) data, this dissertation examined the pathways by which SES, via social, psychological, and behavioral factors predicted physical impairment and overnight hospitalization, and asked whether these indirect relationships differed by race/ethnicity. The HRS is a nationally representative multistage area probability sample administered biennially to respondents over the age of 51 and their spouses. Data collected between 2002 and 2010, covering five waves of the original HRS cohort born between the years 1931 and 1941, were used. Two analysis approaches, Structural Equation Modeling (SEM) and Reconstructability Analysis (RA), were used. Adjustments for the complex survey design were made in the SEM analysis, whereas, data were matched for the RA method using propensity scores.

Results of the SEM analyses supported most of the hypothesized indirect relationships between SES variables and physical impairment via social and psychological factors, but the indirect effect of SES on physical impairment via behavioral factors was weak. Multiple group analyses of path equality using nested chisquare tests indicated that the indirect effect of SES on physical impairment status did not vary by race/ethnicity. Social, psychological and behavioral factors were weakly related 
to overnight hospitalization, and SES was not indirectly related to overnight hospitalization.

While these results supported several hypothesized indirect relationships between SES variables and physical health status, the indirect effect sizes were small. However, because this study examined predictive paths across groups rather than compare mean differences, and because indirect effects are products of individual path coefficients, small effect sizes are not uncommon in mediation analysis. Moreover, over a lifetime, small effects may gradually add up increasing group differences in health status with greater benefits accruing to higher SES individuals via social and psychological factors, as observed in this study.

The RA results showed that indirect relationships between SES and physical impairment were similar across races/ethnicities for identical variables with a few exceptions. In several cases, however, selected SES variables related to social and psychological variables were different for different groups. Cross-sectional indirect relationships were stronger than longitudinal indirect relationships. As in the SEM study, SES was not related to physical impairment via behavioral factors; and, across groups, SES was also not related to overnight hospitalization either directly or via social, psychological or behavioral factors.

Variables predicting physical impairment exhibited differences across groups; these differences were detected because RA, unlike SEM, used disaggregated social, psychological and behavioral factors. Where predictive variables overlapped, the effects 
of identical independent variable (IV) states on physical impairment were similar across groups with a few exceptions.

In summary, both the SEM and RA results indicated that SES was indirectly related to physical impairment via social and psychological factors, and results from both methods also showed that SES was not indirectly related to overnight hospitalization via these factors. SEM did not find that these indirect effects varied by race/ethnicity; RA found a few differences. 


\section{DEDICATION}

To My Parents 


\section{ACKNOWLEDGEMENTS}

I would like to thank my parents for their patience with me throughout the years and for instilling in me a sense of diligence early in my life. Where I was raised, many of my peers did not get the chance to go to school and I am immensely grateful to my parents for the sacrifices they made so I could go to school. To my siblings: thank you for having faith in me and for your patience over the many years that I have not visited you or paid attention to what was going on in your lives.

Thanks to my wonderful committee members who devoted their time reading and correcting the drafts, providing their unique viewpoints and guiding me throughout the process. Special thanks to Dr. Martin Zwick, my dissertation committee chair and my mentor, who patiently guided me throughout the past several years and spent some nights up to help solve some of my analysis problems. Thanks for teaching me to write better. I will always be grateful for your support, mentorship and friendship. Also, special thanks to Dr. Jason Newsom under whom I have learned a great deal about statistical analysis and research methods. The opportunity to work with you was invaluable and I will always be grateful. I am grateful for your responses to all my e-mails and for always being available to guide me through the analysis process and thank you for teaching me to write better. Thanks to Dr. Neal Wallace for the several hours of consultations, for the ideas and recommendations to improve my work. Thanks also to Dr. Kerth O'Brien and Dr. Lindsey Wilkinson, for agreeing to be on my committee and for their invaluable input in my research. 
Over several years, I was mentored by some of the best and generous educators and I will always be grateful for their time. Thanks to Dr. Francis Wambalaba who introduced me to research methods at the United States International University Nairobi, Kenya. Thanks to Dr. Paula Carder (PSU) and Dr. Ana Quiñones (OHSU-PSU) for their support, collaborative research and mentorship. Because of the opportunities you provided, I learned important new skills.

This journey was not easy but it would have been nearly impossible without the constant support of friends, relatives and colleagues. Thanks are due to Dr. Moses Wambalaba and his family who graciously hosted me during the first week in the U.S. and introduced me to PSU the next day I arrived. Special thanks to my aunt Kibirti Zemam and her family for always being there for me and checking up on my progress and well-being. I also express my heartfelt gratitude to my cousin Elilta (Lily) Habtu and my friend Zeccharias Arey for their constant support over the years. Thanks to my friends and colleagues Teresa Schmidt, Zahra Abukar, Senthil Kumar, Peter Geissert, Georgis TMichael, Amanuel Shakir, Rajesh Venkatachalapathy and Amanuel Mehari. Your support at various stages in this journey kept me going and I am grateful for your time and ideas. 
TABLE OF CONTENTS

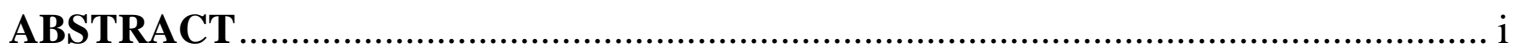

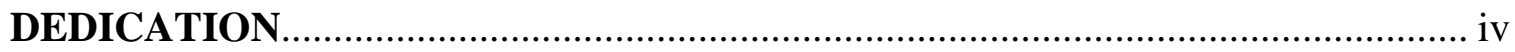

ACKNOWLEDGEMENTS …………………………....................................

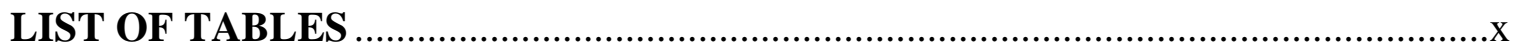

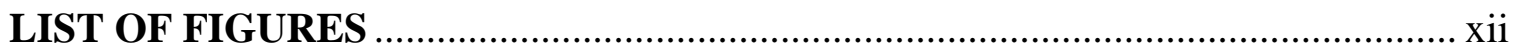

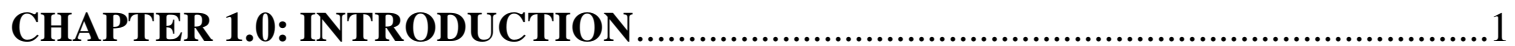

1.1 Overview of Race and Ethnic Composition of the United States Population over

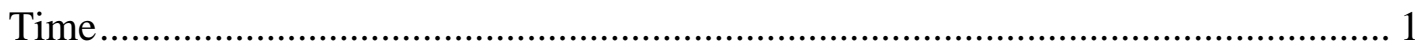

1.2 Health Disparities and Health Disparities Research................................................. 2

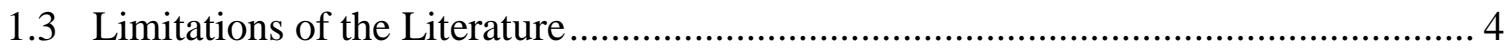

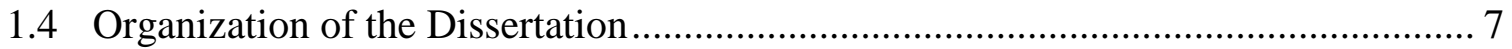

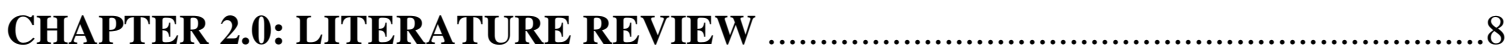

2.1 Socioeconomic Status, Health Status and Health Care Utilization ............................. 8

2.1.1Education, Income, Employment and Wealth ...................................................... 14

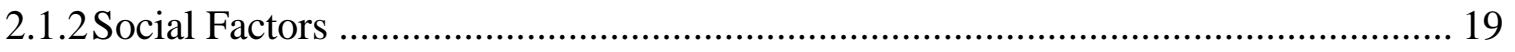

2.1.3Psychological Factors .................................................................................... 24

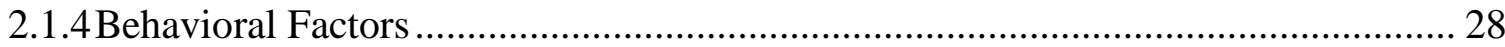

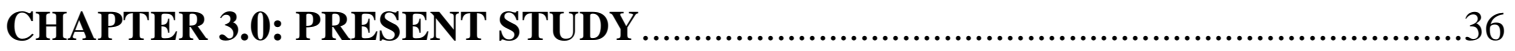

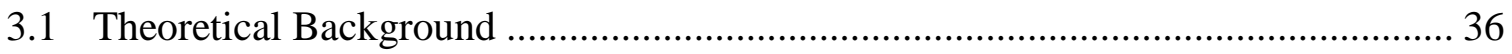

3.2 Conceptual Model and Objectives of the Study ………………............................ 38

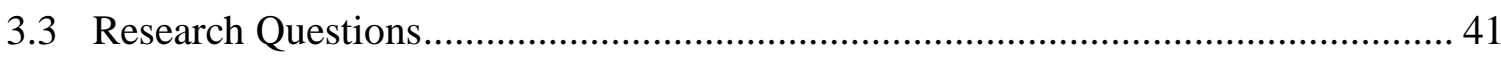

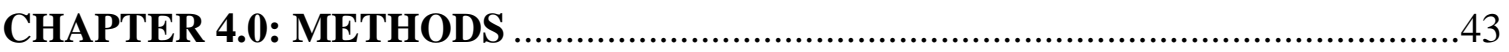

4.1 Data Source, Procedure and Sample................................................................... 43

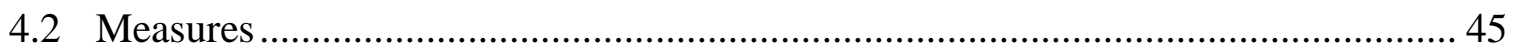

4.2.1 Independent Variables ...................................................................................... 45

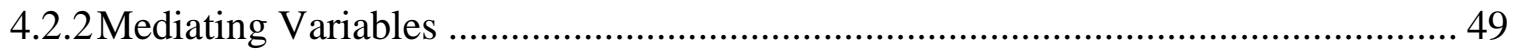

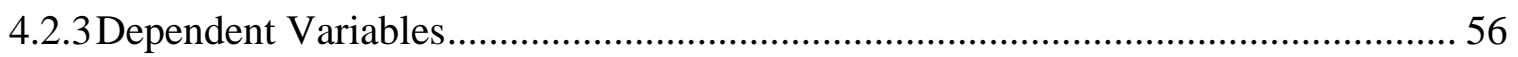

4.3 Analytic Approaches ……………………………………………………….... 57 


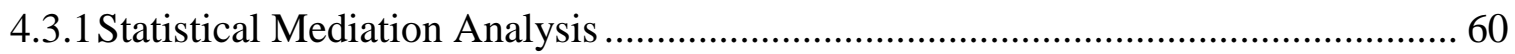

4.3.2Reconstructability Analysis (RA) ....................................................................... 70

CHAPTER 5.0: RESULTS FOR STRUCTURAL EQUATION MODELING ..........81

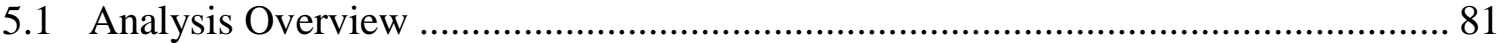

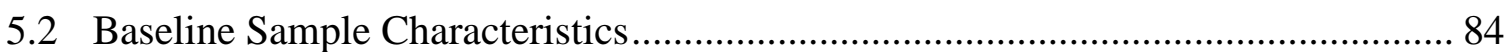

5.3 Mean Scores (or Proportions) of Mediating and Outcome Variables over Time..... 86

5.4 Bivariate Relationships among Study Variables ...................................................... 90

5.5 Summary Results of Significant Indirect Effects for the SEM Method ................... 95

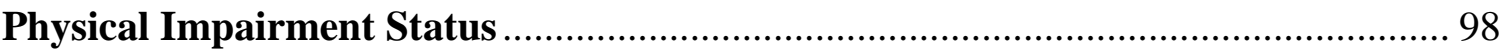

5.6 SES-Social factors-Physical Impairment: Lagged Path Model................................. 98

5.7 SES-Social Factors-Physical Impairment: Growth Curve Model........................... 106

5.8 SES-Psychological Factors-Physical Impairment: Lagged Path Model ............... 116

5.9 SES-Psychological Factors-Physical Impairment: Growth Curve Model ............. 125

5.10 SES-Behavioral Factors-Physical Impairment: Lagged Path Model .................... 132

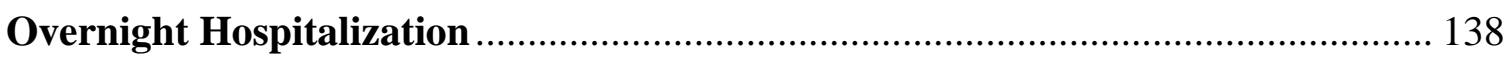

5.11 SES-Social Factors-Overnight Hospitalization: Lagged Path Model ................... 139

5.12 SES-Psychological Factors-Overnight Hospitalization: Lagged Path Model....... 143

5.13 SES-Behavioral Factors-Overnight Hospitalization: Lagged Path Model............ 147

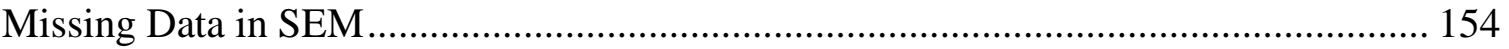

CHAPTER 6.0: RESULTS FOR RECONSTRUCTABILITY ANALYSIS ...........158

Physical Impairment Status ................................................................................... 161

6.1 Cross-Sectional Analysis of Physical Impairment Status........................................ 162

6.2 Cross-Sectional Path Analysis of Physical Impairment Status ............................. 174

6.3 Longitudinal Analysis of Physical Impairment Status .......................................... 183

6.4 Longitudinal Path Analysis of Physical Impairment Status .................................... 191

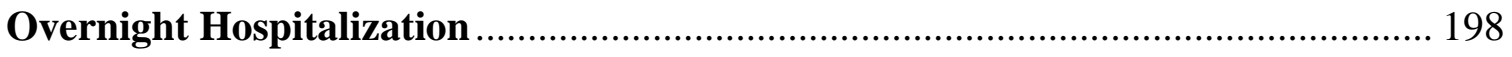

6.5 Cross-Sectional Analysis of Overnight Hospitalization ......................................... 198

6.6 Cross-Sectional Path Analysis of Overnight Hospitalization.................................. 202

6.7 Longitudinal Analysis of Overnight Hospitalization............................................... 204

6.8 Longitudinal Path Analysis of Overnight Hospitalization ..................................... 208 


\section{CHAPTER 7.0: STRUCTURAL EQUATION MODELING: DISCUSSION AND}

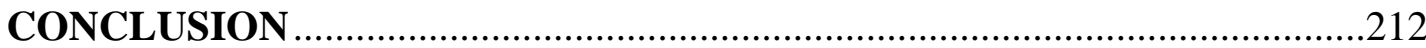

Physical Impairment Status .......................................................................... 212

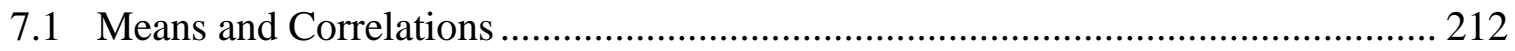

7.2 SES-Social Factors-Physical Impairment Link ………………....................... 219

7.3 SES-Psychological Factors-Physical Impairment ................................................ 223

7.4 SES-Behavioral Factors-Physical Impairment....................................................... 225

Overnight Hospitalization ................................................................................. 227

7.5 SES-Social Factors-Overnight Hospitalization ................................................... 228

7.6 SES-Psychological Factors-Overnight Hospitalization ........................................ 228

7.7 SES-Behavioral Factors-Overnight Hospitalization .............................................. 229

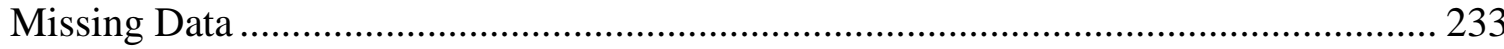

CHAPTER 8.0: RECONSTRUCTABILITY ANALYSIS (RA): DISCUSSION AND

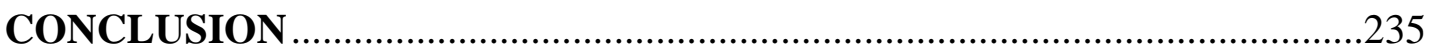

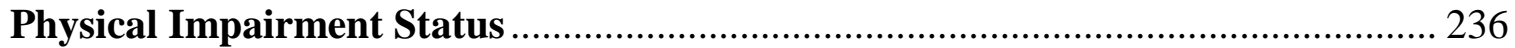

8.1 Cross-Sectional and Longitudinal Analysis of Physical Impairment Status .......... 236

8.2 Cross-Sectional and Longitudinal Path Analysis of Physical Impairment Status .. 248

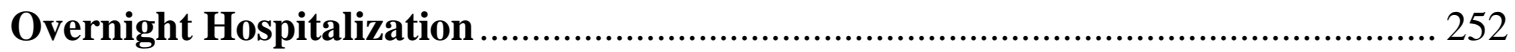

8.3 Cross-Sectional and Longitudinal Analysis of Overnight Hospitalization ............ 252

8.4 Cross-Sectional and Longitudinal Path Analysis of Overnight Hospitalization .... 255

CHAPTER 9.0: CONTRIBUTIONS, LIMITATIONS AND FUTURE STUDY ....257

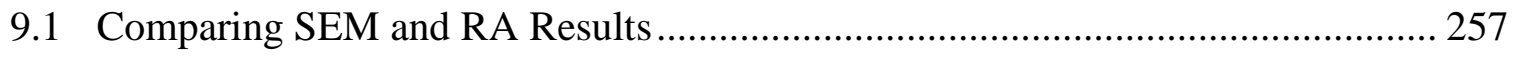

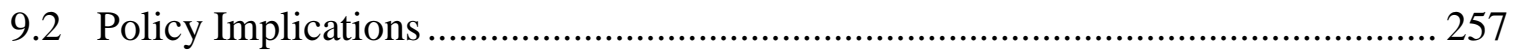

9.3 Contributions of the Present Study to the Literature ............................................. 259

9.4 Advantages and Disadvantages of the Two Analytic Approaches .......................... 263

9.5 Limitations and Future Study ………………………........................................ 265

REFERENCES

Appendix A: Survey Instrument ............................................................................. 291

Appendix B: Additional SEM Results ............................................................................. 295

Appendix C: Additional RA Results.......................................................................... 310 


\section{LIST OF TABLES}

Table 1: Summary of variables used for statistical mediation analysis and RA method.. 48

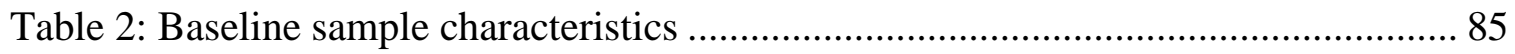

Table 3: Means/proportions for mediating variables and outcome variable at later times 89

Table 4: Bivariate relationships between variables for the Total group ........................... 91

Table 5: Bivariate relationships between variables for the White group ........................... 92

Table 6: Bivariate relationships between variables for the Black group .......................... 93

Table 7: Bivariate relationships between variables for the Hispanic group ..................... 94

Table 8: Summary of significant indirect effects across groups (unstandardized/standardized coefficients). Standard errors are in parentheses .. 97

Table 9: Percent missing data for the physical impairment outcome variable (PI=physical

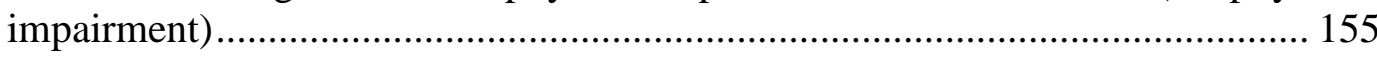

Table 10: Patterns of missing data for physical impairment ( ' + ' $=$ not missing) ........... 156

Table 11: Percent missing data for the overnight hospitalization variable $(\mathrm{OH}=\mathrm{overnight}$

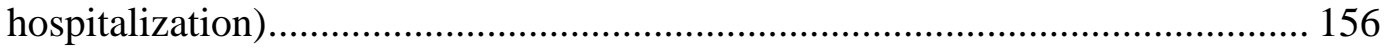

Table 12: Patterns of missing data for overnight hospitalization ('+' = not missing) .... 157

Table 13: Cross-sectional/Longitudinal predictive variables for physical impairment (PI) status

Table 14: Summary of results of inter-race/ethnic comparison of cross-sectional models 163

Table 15: Models with loops for White and Hispanic groups ......................................... 164

Table 16: Fit results for JPI:SomPI for White and Hispanic groups ............................. 165

Table 17: Models with loops for White and Black groups ............................................. 167

Table 18: Fit results for JPI:BmiPI:SomPI for White and Black groups.......................... 169

Table 19: Models with loops for Black and Hispanic groups.......................................... 171

Table 20: Fit results for JPI:BmiPI:SomPI for Black and Hispanic groups .................... 172

Table 21: Uncertainty reductions for cross-sectional path models of physical impairment status

Table 22: Summary of inter-race/ethnic comparison of longitudinal models ................ 183

Table 23: Models with loops for White versus Hispanic groups ...................................... 184

Table 24: Fit results for BmiPI:ExPI:VoPI for the White group ..................................... 185

Table 25: Fit results for SomPI:NegPI for the Hispanic group ...................................... 186

Table 26: Models for White and Black groups ............................................................... 187 
Table 27: Fit results for SomPI:VoPI for White and Black groups ............................. 188

Table 28: Models for Black and Hispanic group ...................................................... 189

Table 29: Fit results for SomPI:NegPI for Black and Hispanic group ......................... 190

Table 30: Uncertainty reductions for longitudinal path models of physical impairment

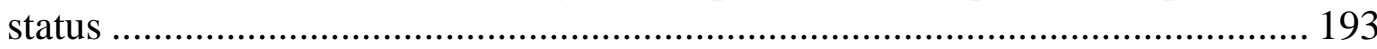

Table 31: Racial and ethnic comparison of cross-sectional models ............................. 199

Table 32: Models with loops for White and Hispanic groups ..................................... 199

Table 33: Fit results for GHo:NegHo for the Hispanic group ...................................... 200

Table 34: Models with loops for Black and Hispanic groups..................................... 201

Table 35: Fit results for JHo for the Black group ................................................ 201

Table 36: Uncertainty reductions for cross-sectional path models of overnight

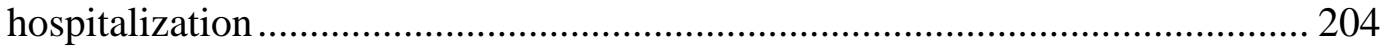

Table 37: Racial and ethnic comparison of longitudinal models................................ 205

Table 38: Models out loops for White and Black groups .......................................... 206

Table 39: Fit results for SomHo for the White group ................................................. 206

Table 40: Fit results for ExHo for the Black group .................................................. 207

Table 41: Uncertainty reductions for longitudinal path models of overnight

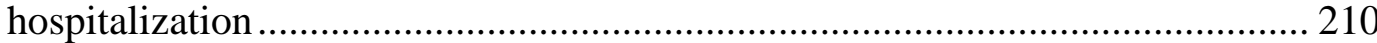

Table 42: SES/demographic, social, psychological and behavioral variables in/not in cross-sectional models predicting physical impairment ................................ 237

Table 43: Partial fit table for unique IV states that increase risk in cross-sectional direct prediction

Table 44: Partial fit tables for unique IV states that increase/decrease risk in cross-

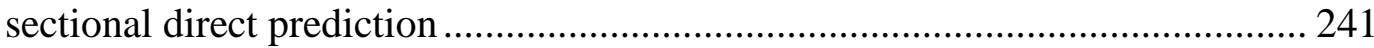

Table 45: Partial fit table for unique IV states that decrease risk in longitudinal non-path models

Table 46: SES/demographic, social, psychological and behavioral variables in crosssectional path models for the physical impairment outcome variable 249 


\section{LIST OF FIGURES}

Figure 1: White, African American/Black and Hispanic/Latino population over time in

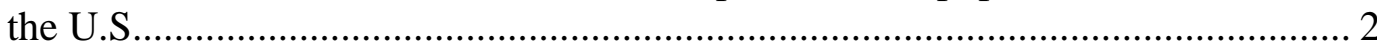

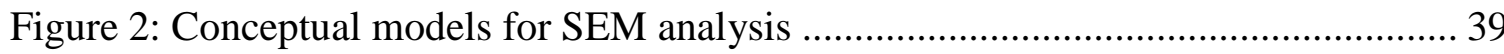

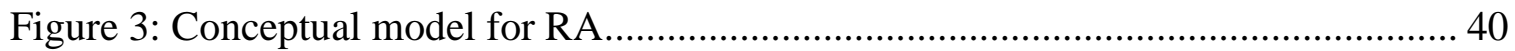

Figure 4: Conceptual model with selected variables in RA path analysis ..................... 41

Figure 5: Total effects of $\mathbf{X}_{\mathbf{1}}$ (SES variables) on $\mathrm{Y}_{3}$ (outcome variable at the third time

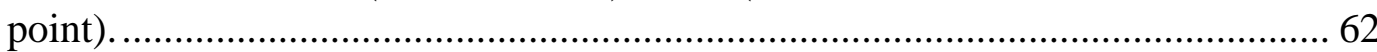

Figure 6: Path diagram and mediation model for a single mediator model, $\mathrm{t}=$ time. ....... 62

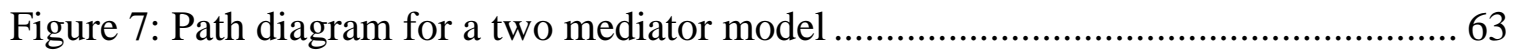

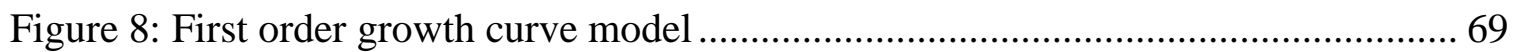

Figure 9: Histogram, Whites (left panel) and Hispanics in each income bin after

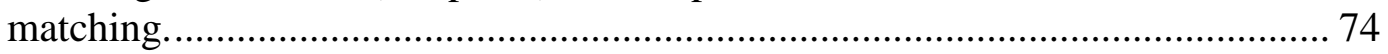

Figure 10: Histogram, Whites (left panel) and Hispanics in each wealth bin after

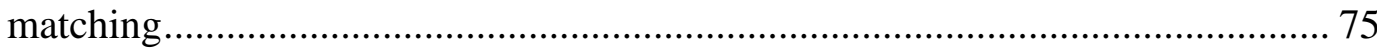

Figure 11: Recursive path model illustrating RA uncertainty reductions........................ 77

Figure 12: Estimated mean change in social integration (SI), physical impairment (PI) and depressive symptoms (DS) level over time............................................ 87

Figure 13: Lagged regression model for Total sample $(\mathrm{N}=7674)$ with standardized path coefficients (Social Integration Mediator) ...................................................... 99

Figure 14: Lagged regression model for Whites $(\mathrm{N}=5717)$ with standardized path coefficients (Social Integration Mediator)

Figure 15: Latent growth curve mediation model for Total sample $(\mathrm{N}=7674)$ with standardized path coefficients (Social Integration Mediator) 108

Figure 16: Latent growth curve model for Whites $(\mathrm{N}=5717)$ with standardized path coefficients (Social Integration Mediator)

Figure 17: Lagged regression model for Total sample (N=7674) with standardized path coefficients (Depressive Symptoms Mediator)............................................... 118

Figure 18: Lagged regression model for Whites $(\mathrm{N}=5717)$ with standardized path coefficients (Depressive Symptoms Mediator)............................................... 120

Figure 19: Lagged regression model for Hispanics $(\mathrm{N}=719)$ with standardized path coefficients (Depressive Symptoms Mediator).....

Figure 20: Second order latent growth curve model for Blacks $(\mathrm{N}=1238)$ with standardized path coefficients (Depressive Symptoms Mediator)..... 
Figure 21: Lagged regression model for Whites $(\mathrm{N}=5717)$ with standardized path coefficients (Behavioral variable Mediators)

Figure 22: Lagged regression model for Whites $(\mathrm{N}=5717)$ with standardized path coefficients (Behavioral variable Mediators)................................................. 151

Figure 23: Cross-sectional path modeling of physical impairment status ..................... 176

Figure 24: Longitudinal path modeling of physical impairment status ........................ 192

Figure 25: Cross-sectional path modeling of overnight hospitalization ........................ 203

Figure 26: Longitudinal path modeling of overnight hospitalization .......................... 208 


\section{CHAPTER 1.0: INTRODUCTION}

\subsection{Overview of Race and Ethnic Composition of the United States Population over \\ Time}

The United States population is becoming diverse with racial and ethnic minorities increasing and the proportion of non-Hispanic White population decreasing over the decades (U.S. Census Bureau, 2013) as illustrated in Figure 1. This changing social structure has implications for public policy including health care workforce education and provision of health care to address disparities in health status and health outcomes. Differences in population health have been observed in homogenous populations in Europe, the United States and around the world. In recent times, apart from social class and socioeconomic status (SES), race/ethnicity has been found to be one of the factors related to health disparities in the United States. A large body of research has demonstrated inequality in health, both by SES, and by race/ethnicity. Because of this

increasing diversity (Figure 1), and continued persistence of health disparities, a variety of policy approaches addressing factors affecting the racially and ethnically diverse population may be necessary. 


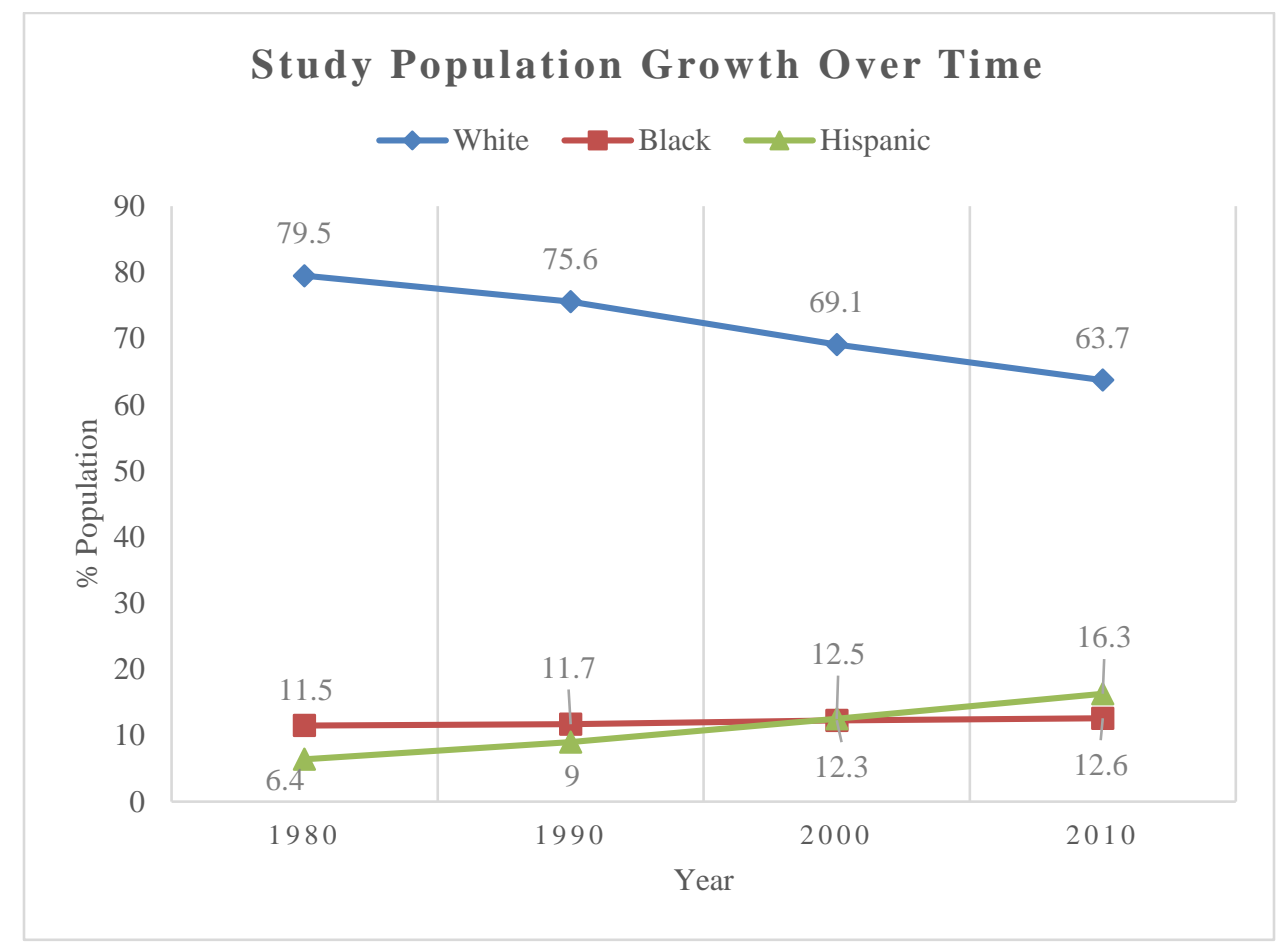

Figure 1: White, African American/Black and Hispanic/Latino population over time in the U.S. Source: Decennial census of population, 1940 to 2010

\subsection{Health Disparities and Health Disparities Research}

Health disparities refer to differences in health outcomes among various groups of the population, based on race, ethnicity, gender, education, income, occupation, disability, geographic location or sexual orientation (U.S. Department of Health and Human Services, 2000). Alternate definitions and perspectives of health disparities also exist (Braveman, 2006; Institute of Medicine, 2006; Whitehead, 1992), but the generality of the DHHS characterization of health disparities made it most appropriate for use in this study. Socioeconomic status (SES) is closely associated with race/ethnicity where nonHispanic Whites fall in higher SES position. The four constituents that have been used to represent SES include income, education, wealth, and employment status. These SES constituents, to a large extent, determine social structures that affect individual well- 
being. In this study, SES refers to the four separate variables including total household income, total wealth less debt, education level, and employment status.

The role of macro-social factors in health outcomes saw renewed interest in the early and mid-1990s. Several studies (House, Kessler, \& Herzog, 1990, Link \& Phelan, 1995) proposed social conditions as principal causes of diseases and unequal distribution of adverse health outcomes among racially and ethnically diverse populations. A large body of research refocused attention on the remote social and economic health risk factors, showing that SES was consistently associated with a variety of health status measures. Health status and health disparities research has often emphasized on proximal risk factors such as individual behaviors, or remote risk factors such as SES factors, placing the burden on individuals to adopt healthy behaviors while discounting the constraints that institutional and social structures place on individuals. These approaches have limitations because proximal and distal risk factors are likely interrelated, and the sources of immediate risk factors are rooted in remote risk factors (Williams \& Jackson, 2005) with potential interaction effects. Research that focuses primarily on immediate risk factors may appear to disregard remote risk factors, shifting the onus of health status to individuals regardless of the environmental context that shapes opportunities and hazards for various groups of the population. Policies based only on remote or immediate health risk factors will likely be less effective than policies that address both. Thus, healthcare and health services researchers advocate for research and policy approaches that include both remote and immediate risk factors as well as the interactions of these 
risk factors that may better account for the observed race/ethnic differences in health outcomes (Angell, 1993; Adler et al., 2008; 1994; Adler \& Conner Snibbe, 2003).

\subsection{Limitations of the Literature}

Several theoretical orientations have guided health disparities research but the majority has been focused on variations within a homogeneous racial or ethnic group. The theory of social causation, first advanced by Giddings (Giddings, 1904), and later expanded and used in empirical research (Dohrenwend, 2000; Johnson, Cohen,

Dohrenwend, Link, \& Brook, 1999; Kaniasty \& Norris, 2008), much of it regarding mental health, views causes of differences in population health as originating from the general social environment such as disadvantages and adversity in the social environment. These studies have routinely used socioeconomic and demographic variables to predict health status, presuming that social level variables such as material conditions preceded (and caused) decline in health status.

Others have proposed the theory of social selection which advanced the view that deterioration in health precedes decline in material conditions (i.e., poverty). Both theoretical orientations appear to have merits in different settings. In large scale population studies, the theory of social causation is more relevant. One can imagine situations in which deterioration in health precedes decline in SES status that individuals fail to overcome but this may not be characteristic of the general population. The social selection hypothesis indicated that some studies have used health status to predict SES (Mulatu \& Schooler, 2002) and have found that health status did not predict SES as 
strongly as SES predicted health status, suggesting that in a national scale study, the primary direction of influence was from SES to health status, and that this relationship varied by race/ethnicity. The mechanisms or pathways by which SES affects differential health status by race and ethnicity are not clear, however.

Race/ethnicity and SES variables predict differences in health outcomes in the United States but race/ethnicity or SES variables alone do not completely account for the observed differences. Socioeconomic status, race, ethnicity and several other demographic variables are correlated (LaVeist, 2005) with minorities occupying lower positions in the socio-structural hierarchy. Higher education achievement, high income and wealth levels, and being employed among other resources are stratified along racial, ethnic and gender identities (Adler \& Conner-Snibbe, 2003; Myers, 2008). Further, for similar educational achievement and occupational status, minorities and females earn lower income and are, thus, less wealthy relative to White males, an inequity that perpetuates inequality throughout life. Inequality in SES is consistently related to health status and health outcomes. The body of research on health disparities or inequalities by race/ethnicity does not emphasize the mechanisms or pathways by which inequality in SES gives rise to disparity in health status. Therefore, further research to uncover the mechanisms by which SES is related to health status and health outcomes is essential (Adler \& Conner-Snibbe, 2003; Adler \& Rehkopf, 2008; Angell, 1993).

Conceptually, SES is believed to influence health status through intervening variables such as social, psychological and behavioral variables (Angell, 1993; Myers, 
2008). However, whether and how social, psychological and behavioral variables play as intermediate variables in the pathways between SES and health status or health outcomes by race/ethnicity is an area that is yet to be thoroughly examined. Research examining the various pathways by which SES may be related to health status and health outcomes, generating health disparities among diverse racial and ethnic population is important.

Several conceptual models have been proposed to illustrate the possible pathways by which SES may influence health status and healthcare utilization and how these relationships may differ by race/ethnicity resulting in disparities in health outcomes (Adler \& Snibbe, 2003; Adler \& Ostrove, 1999; Cwikel, Dielman, Kirscht, \& Israel, 1988; Myers, 2008). These pathways included social, psychological, and behavioral factors as intermediate variables. The conceptual models are complex involving a long chain of causal effects, interaction effects, and feedback effects, and do not lend themselves to empirical evaluation with current techniques and available data. Analysis of hypotheses arising from these conceptual models has, thus, been limited either to a part of a conceptual model or to a modified version (Myers \& Huwang, 2004) of a conceptual model which may be analytically more tractable. A multiple group analysis comparing differences in pathways between SES and health status is one of several approaches that can contribute to the understanding of the mechanisms by which SES is indirectly associated with health status via social, psychological, and behavioral factors. Unpacking the role of social, psychological, and behavioral variables as mediating factors has the potential to increase understanding of how SES variables may predict health status indirectly and can inform policies targeting the specific processes that shape health 
statuses for racially and ethnically diverse older Americans. The aim of this study was to examine and compare the indirect effects of SES on health status by race/ethnicity among older Americans.

\subsection{Organization of the Dissertation}

The rest of the dissertation is organized as follows. Chapter 2 provides review of literature on health disparities by socioeconomic status and by race and ethnicity. Chapter 3 provides conceptual models that motivated this study, objectives of the study, theoretical framework for this study, and research questions. Chapter 4 provides research methods, research design, data source, sample and sampling design, and analytic approaches. The two distinct analytic approaches used in this study, namely Structural Equation Modeling (SEM), based on statistical mediation analysis, and Reconstructability Analysis (RA), based on information and graph theories, are discussed. Chapter 5 provides SEM results and Chapter 6 provides RA results. Discussion and conclusions for SEM results and RA results are provided in Chapter 7 and Chapter 8 respectively. Finally, Chapter 9 discusses contributions to the literature, advantages and disadvantages of the two analytic approaches used, limitations of this study and recommendations for future study. 


\section{CHAPTER 2.0: LITERATURE REVIEW}

\subsection{Socioeconomic Status, Health Status and Health Care Utilization}

Socioeconomic status (SES) is conceptualized to represent the individual's position in socio-structural hierarchy which potentially determines access to resources that restore, enhance and maintain health. The key traditional measures of SES include income, education and occupational status (Adler \& Newman, 2002; Antonovsky, 1968; Bowen, 2010; Phelan \& Link, 2005), but wealth has also been used as one of the key SES indicators in some studies (Ostrove, Feldman, \& Adler, 1999). Socioeconomic status is inversely related to morbidity and mortality, where, minorities and individuals in lower SES positions experience higher morbidity and mortality rates (Agency for Healthcare Research and Quality, 2013; Angell, 1993; Ferraro \& Shippee, 2009). Further, race/ethnicity and SES are correlated where minorities rank lower and SES accounts for substantial racial/ethnic disparities in morbidity and mortality (Krieger et al., 1999; Williams et al., 2012). Disparities in health status and healthcare utilization have been observed among minorities, particularly among non-Hispanic Blacks, Native Americans and Hispanics compared to non-Hispanic Whites. This suggests that SES is a key source of differences in health outcomes by race/ethnicity. Other sources cited for the observed differences in health status include institutional racism, real or perceived discrimination against minorities, lack of insurance and low quality of care for individuals covered by Medicare and Medicaid as well as differential approach to treatment (LaVeist, 2005; Schneider, Zaslavsky, \& Epstein, 2002). It is important to note that consequential social systems in the United States were created by the majority (non-Hispanic Whites) group 
over time, especially by those who controlled resources and made decisions affecting various realms of life, to address their needs based on cultural arrangements while systematically discounting the experiences, needs and cultures of racial and ethnic minorities.

SES variables including education, income and employment status determine access to healthcare resources and enable participation in health promoting behaviors (Link \& Phelan, 1995) such as preventive screening, early detection and treatment of diseases. Lower SES, including lack of stable employment or chronic unemployment which is related to education level, are reasons for the absence of employment based health insurance (National Research Council, 2003), which disproportionately affects racial and ethnic minorities. Lower participation in preventative healthcare activities is, therefore, partly related to lack of insurance. Any single SES variable (i.e., education, income, wealth or occupational status) fails to account for the total observed differences in health outcomes among races/ethnicities in the United States (Cook \& Manning, 2009; Green et al., 2007); indeed, all SES variables together do not totally account for the observed differences. The limitation of most of the studies has been the use of a single variable to capture the complex relationships of SES to health. Several scholars advocate using as many SES variables as possible including wealth because of the unique contributions of each measure to explaining observed race/ethnic differences in health outcomes (LaVeist, 2005; Ostrove et al., 1999; Williams \& Collins, 1995). Other studies show that additional sources, including daily experiences of racism, discrimination and associated stress burden that racial and ethnic minorities carry, specifically Blacks and 
Hispanics, may account for part of the observed race/ethnic disparities in health (Williams et al., 2012).

Remaining in lower SES position for extended period of time strains individual's physiological regulatory processes and people with continued economic hardships are more likely to experience difficulties with activities of daily living (ADLs), that is, develop physical impairment (Lynch, Kaplan, \& Shema, 1997). Of the three race/ethnic groups considered in this study, the Urban Institute and Kaiser Commission on Medicaid and the Uninsured (Urban Institute and Kaiser Commission on Medicaid and the Uninsured, 2012) reports national poverty levels in 2011-2012 among non-Hispanic Blacks as the highest (35\%) followed closely by Hispanics $(33 \%)$ which were more than double the rates in non-Hispanic Whites (13\%), a trend that persisted over time. Socioeconomic factors also shape the creation and maintenance of social networks, psychological status, and health behaviors (Kaplan, 1995) mainly because of higher SES individual's ability to afford the resources to meet relationship needs, engage in healthy behaviors and better cope with psychological stressors. These studies establish associations between SES and psychosocial factors and between SES and behavioral factors by race/ethnicity using different study samples. Some racial/ethnic differences in health status and health outcomes, therefore, may arise from poor social integration, increased psychological distress and sedentary lifestyles which are associated with lower SES. Analysis of the hypothesized relationships of the pathways from SES to health status, as well as from SES to health care utilization, in a racially and ethnically diverse population can enhance understanding of racial/ethnic differences in health. Examination 
of pathways from SES to health status through other intermediate variables within a single study is considered necessary in advancing understanding of the mechanisms by which SES predicts health over time (Adler \& Conner-Snibbe, 2003).

Extensive experimental and quasi-experimental studies on animals and humans (Cohen, 1988; Cohen, 2004; Cohen \& Wills, 1985; Sapolsky, 1998) showed that ranking lower in the social hierarchy increases susceptibility to diseases through rapid stimulation of the endocrine systems to adapt to environmental threats. Absolute as well as relative deprivation of resources creates vulnerabilities to diseases, but in the United States, the sources of vulnerabilities and differential health status appear to be primarily due to relative deprivation (Sapolsky, 2004). Health promoting psychosocial resources that mediate the effect of SES on health status are differentially distributed according to social class (Taylor \& Seeman, 1999). That racial and ethnic minority generally fall in lower SES positions means that these groups would lack resources to mitigate environmental hazards to their health, leading them to engage in unhealthy behaviors to obtain temporary relief from stressful conditions (Jackson, Knight, \& Rafferty, 2010). Further, race/ethnic disparities in health have been observed among non-Hispanic Blacks and nonHispanic Whites with similar SES position, indicating the possibility that institutional policies and the feelings of group superiority/inferiority in social hierarchy contribute to race/ethnic differences in health (Williams, 1999) through various physiological process that last over the life course. Social systems in the United States tend to disadvantage minorities in at least two ways regarding mobility in social hierarchy. First, the factors that disadvantage minorities include implicit institutional barriers to education due to lack 
of resource allocation, implicit discrimination in employment and lower rate of return upon employment for equivalent educational achievement. This is also true for women and other minorities with the possible exception of Asian Americans. Second, healthcare institutional policies and system structures limit minorities' ability to access care because of provider location, physician-patient cultural differences and thus difficulty in communication, lower quality of care and differential treatment plans (Agency for Healthcare Research and Quality, 2013; Institute of Medicine (US) Committee on Quality of Health Care in America, 2001). The confluence of these factors gives rise to higher risks for morbidity and mortality in minority groups.

Physical impairment status. Physical impairment status is one of the crucial outcomes of other domains of people's lives and well-being including their socioeconomic status (Bunker, Gomby, \& Kehrer, 1989; Maddox \& Clark, 1992; Zimmer \& House, 2003). The physical impairment outcome variable in this study was measured by activities of daily living (ADL) index. The index indicated whether respondents had limitations in performing basic activities necessary in their daily lives such as bathing, dressing, going to toilet, getting in and out of bed, feeding, and walking across the room (Fonda \& Herzog, 2004). Originally developed in early 1960's to study treatment results among the elderly (Katz, Ford, Moskowitz, Jackson, \& Jaffe, 1963), the ADL grades have been used extensively to summarize physical performance of necessary activities among older people. Physical impairment, that is, inability to perform the five ADL measures, gradually increases over time. Increase in physical impairment may, however, vary by SES and by race/ethnicity within a cohort because higher SES may enable 
individuals to engage in activities or take actions that delay the onset and rate of increase in physical impairment.

Overnight hospitalization. Medical care use differs by race, ethnicity and SES. Minority's access to primary care tends to be limited and barriers may raise rates of overnight hospitalization due to lack of access to preventative care. Similarly, wealthier people may have access to medical care and may use resources for procedures that could be addressed in ambulatory care setting. Preventable hospitalizations and overnight hospitalizations in general are likely to be higher among racial/ethnic minorities particularly among non-Hispanic Blacks, Hispanics and other minorities, however. Some studies (Gaskin \& Hoffman, 2000) found that Hispanics and Blacks were hospitalized more often than non-Hispanic Whites, and attributed this disparity to lack of access to primary care. Other studies (Eggers \& Greenberg, 2000), found that among all races/ethnicities, for almost all causes, Blacks and Native Americans had the highest hospitalization rates, which mirrored mortality rates that were generally higher for minorities with the exception of Asian Americans. Access to primary care allows for early diagnosis of chronic diseases and early medical intervention resulting in better disease management. Using hospital discharge data, Mayr and colleagues (Mayr et al., 2010) found higher age- and sex-standardized racial differences in severe sepsis concluding this to be a result of elevated risk of organ dysfunction among Blacks more than Whites. These studies suggest that non-Hispanic Blacks, Hispanics and other minorities, apart from Asian Americans, experienced higher rates of hospitalizations compared to their White counterparts. Such disparities, thus, necessitate evaluation of the 
processes that may account for disparities in overnight hospitalizations by race/ethnic groups.

\subsubsection{Education, Income, Employment and Wealth}

Education. Education is one of the key constituents of SES measures (Adler \& Newman, 2002) and one of the most studied variables (Kaufman \& Cooper, 2001; Williams, Yu, Jackson, \& Anderson, 1997). Studies suggest that higher education may confer coping skills and strategies that can be deployed during adverse life events (Pearlin \& Schooler, 1978) apart from the potential for earning higher income. Education is associated with higher income, and higher incomes enable individuals to access healthy diet, resources for physical activity, and afford leisure time for such physical activities as well as live in better residential neighborhoods (Diez Roux \& Mair, 2010). But this scenario may not be true across race/ethnic groups because educational return for minorities is not the same as that for non-Hispanic Whites as indicated in the above section. Minorities, specifically Native Americans, non-Hispanic Blacks and Hispanics have been and continue to be marginalized, where residential areas, access to education and economic circumstances remain less than ideal (Williams \& Jackson, 2005). Longer years of education are associated with better health status (Mirowsky \& Ross, 2003) and lower premature mortality rates (Pappas, Queen, Hadden, \& Fisher, 1993) as individuals with advanced education gain access to and use information to obtain medical care for better recovery from illnesses and better maintenance of health. Individuals in lower SES and disadvantaged race/ethnic groups are less likely to engage in health practices, and changes in health promoting practices over time are stratified by race/ethnicity and SES 
(Williams \& Jackson, 2005). Benefits of education in successfully coping with lifestyle changes, maintaining supportive relationships, and avoiding unhealthy habits over the life-course, likely play important role in health status differences.

Benefits of education also spill-over to offspring. For instance, individuals whose fathers had less or equal to eight years of education had $11 \%$ higher odds of coronary heart disease (Bowen, 2010), one of the leading causes of impairment and death in the United States. To a large extent, education as one of the SES components determines income including income of one's children. Kuh and Shlomo (2004) found that father's social class independently predicted male midlife earnings. Although their study focused on racially homogenous population these relationships likely occur across racially/ethnically diverse populations. Educated parents influenced children's educational achievement, skill development, and provided financial backing and social contacts to succeed in life (Bowen, 2010), which might explain the reasons minorities particularly African Americans score lower in most SES and health indicators and appear unable to escape this cycle. People who stay in unfavorable socioeconomic position for a long period of time experience higher morbidity and mortality risk. One route out of lower SES position is education which is considered one of the pillars of SES indicators. However, education alone does not account for race/ethnic differences in health suggesting the importance of other variables including income, wealth and occupational status. 
Income. Income is another measure widely used as one of the indicators of SES. Although studies frequently use income as a measure of SES, some studies (Kaplan, Haan, Syme, Minkler, \& Winkleby, 1987; Oliver \& Shapiro, 2006) argue that income as well as education and occupation are generally poor indicators of SES for various reasons and particularly among older individuals. One argument against the use of income and education as measures of SES is that education follows gender and race/ethnic divide where White men realize the greatest return on education compared to other races/ethnicities and females. Another argument is that income is a weak indicator of SES among older Americans because of their transition to retirement and subsequent loss of job based income. Thus, income becomes a less sensitive measure after the age of 65 (Kaplan et al., 1987). However, levels of income and wealth are not necessarily similar across groups in retirement because of differential investments during one's working years as well as differential wealth accumulation. Most of the observed racial disparities in health can be explained by socioeconomic status, but, at similar income levels, racial disparities in health persist due to facially friendly but systematically discriminatory institutional policies (Williams, 1999).

Employment. One way of measuring occupation is to simply determine whether one is employed or unemployed (Adler \& Newman, 2002), but a fine-grained classification of job status provides clues to deeper understanding of the effects of various job grades (Marmot et al., 1991). Job status as well as job loss are related to depressive symptoms, social integration and health behaviors. The employed have better health status than the unemployed (Ross \& Mirowsky, 1995) but at least part of the effect 
of occupational status on health status is believed to be indirect through broader social environment including social networks, behavior, and access to health services (Angell, 1993).

The National Research Council (National Research Council, 2004) suggested that SES affects health indirectly through several mechanisms including opportunities, choices and behaviors related to availability of resources across one's life-course. As described above, non-Hispanic Blacks and Hispanics have relatively fewer resources at their disposal to create and take advantage of opportunities. Bromberger and Matthews (1994) found that initially unemployed women exhibited higher levels of depressive symptoms and that these symptoms decreased in women who were later employed. The gain was greater for those with longer years in school and supportive marital status than those with difficulties in marital relationships and had lower education, further pointing to positive inter-correlation between SES variables. Involuntary unemployment acts as a stressor but also impedes one from engaging in health behaviors because of the lack of resources.

Wealth. As indicated above, individuals may increasingly depend on accumulated wealth to meet growing health needs later in life but distribution of wealth is skewed in favor of non-Hispanic Whites because of higher earnings and higher return on investments over the life-course. Wealth, then, is considered a better indicator of individual position in the social hierarchy (Oliver \& Shapiro, 2006) especially among the older population. LaVeist (2005) goes further to suggest that net worth may function as 
the single most comprehensive measure of socioeconomic status because it captures other aspects of SES including intergenerational transfer of wealth and recommends the use of multiple measures of SES whenever possible.

Racial/ethnic disparities in levels of accumulated wealth in American society are substantial and the wealth gap between non-Hispanic Whites and other racial minorities persists. Using the Health and Retirement Study, Choudhury (2002) showed that significant gaps in wealth exist between races and ethnicities that begin at birth and endure over the life-course. Median net worth of households in 2005 was $\$ 134,992$ for non-Hispanic Whites, \$18,359 for Hispanics and \$12,124 for non-Hispanic Blacks; and in 2009 , net worth was $\$ 113,149$ for non-Hispanic Whites, $\$ 6,325$ for Hispanics and $\$ 5,677$ for non-Hispanic Blacks demonstrating the persistence of skewed resource distribution in the United States (Taylor, Kochhar, Fry, Velasco, \& Motel, 2011). When used with other SES variables to predict health status, wealth makes a unique and significant contribution to explaining health status differences among Whites and Blacks (Ostrove et al., 1999) in addition to variations accounted for by other SES variables. Thus, the assertion that relative wealth disparity is reflected in health status disparities as well as disparities in health care utilization appears to be sound.

Using total wealth as one of the indicators is essential especially in postretirement period, because income may not be a strong indicator of SES after the age of 65 due to retirement and consequent loss of income (Kaplan, et al., 1987). Beyond the age of 65, Americans may rely on accumulated wealth to maintain health and those who 
accumulated more wealth may be well positioned to supplement social security and other sources of income.

\subsubsection{Social Factors}

There has been considerable interest in the study of social relationships, including social integration (Cohen, 2004) and their potential supportive function in promoting and maintaining health. Conceptualizations, definitions and measures of social relationships vary (House, Umberson, \& Landis, 1988). Studies have employed terms such as social ties, social connections and social relationships interchangeably (Berkman, Glass, Brissette, \& Seeman, 2000), but these terms vary, both conceptually and in the metrics used to measure them. Nonetheless, social connections are believed to provide the basis for supportive benefits. Support benefits have been frequently conceptualized as being comprised of four components: emotional, appraisal, instrumental and informational (Berkman, et al., 2000). Earlier, a slightly different conceptualization of social support proposed the dimensions of social support as emotional support, informational support and tangible support (Schaefer, Coyne, \& Lazarus, 1981). An important aspect of the study of social relationships is to understand the broader upstream factors such as SES that shape social network structures for various race and ethnic groups.

In some studies, social ties or social connections have been distinguished as primary vs secondary (Thoits, 2011), while, in other studies, strong vs weak ties (Granovetter, 1973) were conceptualized. Primary or strong ties reflect groups such as family, friends and relatives whereas secondary or weak ties reflect more formal ties at work, religious organizations, and interest groups including volunteering in charitable 
organizations (Thoits, 2011). These weak and strong ties form a network that becomes important in the individual's interactions in the social context where the network's benefits may be utilized.

Social integration is conceptualized as a phenomenon embedded in the broader social network, and race/ethnicity and SES are presumed to affect the nature of social networks of individuals. Brissette, Cohen, and Seeman (2000) define social integration as participation in various forms of social relationships that individuals engage in, such as, membership in organizations, interactions with friends, relatives and neighbors. Existence of beneficial social relationships or networks are presumed to buffer against effects of stressors but even in the absences of stressors, social integration is beneficial to health (i.e., connectedness irrespective of existence of stress is beneficial to health) (Cohen, 2004).

Social integration is sometimes viewed as social connectedness (Berkman et al., 2000) and measures of social integration vary. This study relied on Berkman's conception of Social Network Index (SNI) as a measure of social integration. Berkman's SNI takes in to account other social relationship measures including social participation measures, perceived integration measures, and role-based measures (Cohen, Underwood, \& Gottlieb, 2000). The SNI incorporates contact with friends and family, church membership and group membership (Berkman \& Syme, 1979) and later evolved to include marital status, interactions with friends, relatives, children, and membership in formal or informal community, religious and interest groups (Berkman et al., 2000; 
Berkman \& Syme, 1979; Seeman, Bruce, \& McAvay, 1996; Seeman, 1996; Taylor \& Seeman, 1999). Volunteering fits in this category as a measure of social integration and was included in the index. Berkman and colleagues (Berkman et al., 2000) proposed that upstream factors such as SES condition social network structure and social integration is embedded within this network structure. Existence of social ties, that is, presence of structure does not guarantee beneficial utility of the network (House, Umberson, \& Landis, 1988) but absence of a network most likely entails absence of support.

Cobb (1976) and Cassel's (1976) extensive review of published studies on the association between social relationships and health status provided a new impetus to the theory that social relationships were protective of health. Several studies (Berkman \& Syme, 1979; Cohen \& Wills, 1985; House et al., 1988) found association between social ties and mortality, where the most isolated individuals tended to have higher morbidity and mortality risk. Having a diverse social network tends to improve resistance to diseases. For instance, Cohen, Doyle, Skoner, Rabin, and Gwaltney (1997) tested the relationship between social network diversity and susceptibility to the common cold and found an inverse relationship between social network diversity and susceptibility to the common cold indicating the protective effects of social network. In the British White Hall II study, Stringhini and colleagues (Stringhini et al., 2012) also found that marital status and network score were related to mortality, where, those who were married and with higher network score had lower risk of mortality. 
Social isolation, which is the opposite of social integration, is a risk factor for mortality (Berkman \& Syme, 1979; House et al., 1988; Stringhini et al., 2012). However, factors that promote social integration are not well understood. House, Landis and Umberson (1988) noted that social relationship factors have not been studied as dependent variables. It is not well known why or how SES predicts social integration in various race/ethnic groups or even in a racially/ethnically homogenous population. Several studies speculate that formation and maintenance of beneficial relationships may be attributed to broader environmental factors such as overcrowded and crime susceptible neighborhoods and SES factors including wealth, income, education and employment status.

Socioeconomic status tends to influence the nature of an individual's social relationships. Some studies (Berkman \& Syme, 1979; House et al., 1988; McLeod \& Kessler, 1990; Taylor \& Seeman, 1999; Whelan, 1993) found that higher SES individuals tend to have qualitatively better and quantitatively more relationships that help promote and maintain well-being by enhancing the networks' resources and availability of these resources to the individual. Availability of resources enables higher SES individuals to meet support demands without considerable strain on psychosocial resources. Because racial/ethnic minorities are more likely to fall in lower SES, they are more likely to lack quality relationships that promote health status. Further, studies (Bradley \& Corwyn, 2002; Conger \& Donnellan, 2007; Evans, 2004) have found an inverse relationship between household income and social conflict (i.e., negative social interaction) within a family such that increase in household income was directly related to decrease in 
negative interaction (conflict) but such findings may have to be viewed contextually within cultural practices and expectations. Support expectations can lead to tension or conflict when those expected to provide the support lack adequate resources to meet the demand for support. Fewer years of education and lower income are associated with higher psychosocial risk factors such that lower SES people report lower levels of social relationships and support (House \& Williams, 2000). Because SES is stratified along race/ethnicities in such a way that Blacks and Hispanics rank lower, social relationships may vary among these sub-groups as a result. The nature of social relationships also varies based on geographical location, educational level and professional status.

Geographical factors of networks are bound to limit proximate interactions with network members. Some studies (Ajrouch, Blandon, \& Antonucci, 2005) found that professional men had geographically diverse network which may limit personal contacts including getting together for events. Occupational status, similarly, affects the nature of relationships for men and women. Higher education was not associated with close personal friends; however, individuals with more years of education had larger network (Ajrouch et al., 2005). The findings of the Ajrouch and colleagues' study were limited to a homogeneous (White) sample. Although the conclusions cannot be extended directly to race/ethnic minorities in the United States, their study illuminates the nature of networks that people at varying SES levels establish.

The foregoing discussion indicates associations between SES and social relationships, and between social relationships and health status. However, the processes 
by which the broader macro-social factors facilitate or deter the formation of social networks within which social integration is embedded are not well known (Berkman, Glass, Brissette \& Seeman 2000). Studies examining the association between SES and social relationships, where, social relationships are used as dependent variables are few. But some studies demonstrated the correlations between social affiliations and social statuses, and between social status and economic inequality (Kawachi, Kennedy, Lochner, \& Prothrow-Stith, 1997; Wilkinson, 1999)

On broader macro-social and economic measures such as income, education, networth, employment status and the nature of residential neighborhoods, racial minorities are disadvantaged. Therefore, race/ethnic minorities are expected to score poorly on social integration measures including marital status and association with secondary groups that could be beneficial to accessing employment opportunities. Quality of social relationships rather than quantity has been associated with better self-rated health status (Pinquart \& Sörensen, 2000), a characteristic that may vary by race/ethnicity.

\subsubsection{Psychological Factors}

One measure of psychological factors is the Center for Epidemiologic Studies Depression (CES-D) scale. The present study uses the term depressive symptoms for psychological factors. Prevalence of depressive symptoms varies by socioeconomic status as well as race/ethnicity. Studies show that socioeconomic status is inversely associated with depressive symptoms (Lorant et al., 2003), and depressive symptoms are positively linked to physical impairment status (Ormel, Rijsdijk, Sullivan, van Sonderen, \& 
Kempen, 2002). African Americans experience faster decline in physical health status over time compared to non-Hispanic Whites. This may be due to resources available to non-Hispanic Whites that enable them to slow down physical impairment at a later age by avoiding or properly addressing stressors. Race/ethnic minority's disadvantage tends to last the entire life course where economic disadvantages begin long before an individual is born. The effects of these socioeconomic challenges are later compounded with implicit, real and perceived racial/ethnic stereotyping that degrades psychological wellbeing.

Adaptation to hostile environments and hypervigilance, which are common among the urban poor and minorities because of residential environments, are costly to one's health. Stressors stimulate physiological processes that regulate neuroendocrine functions designed to safeguard individual's health integrity but over stimulation of these regulatory functions to counter stress can have negative impact in the long run (McEwen, 1998). The natural response to adapt to perceived hazardous environments and extended hyperactivity of the regulatory systems of the body lead to pathologies and this constant adaptation to maintain stability in the face of change was called allostasis (Sterling \& Eyer, 1988). McEwen \& Stellar (1993) proposed that the frequent and inefficient activation of these adaptive systems, or allostatic load, harm the normal functioning of the human adaptive system leading to chronic diseases. The price of constant effort to adapt to unfolding adverse experiences among race/ethnic minorities and the poor, therefore, may be the observed disparate health outcomes and early deaths among minorities and the poor in national scale studies. 
Lack of control over factors affecting one's life is also associated with allostatic load, and poor people and minorities appear to be most disadvantaged in this regard. Clearly, racial/ethnic minorities are disproportionately affected by worse health outcomes in addition to being perceived as inferior, which is associated with systemic discrimination. This dual disadvantage exposes minorities to unfavorable socioenvironmental conditions. Studies suggest that elevated levels of depressive symptoms are risk factors that accelerate onset of chronic diseases including cardiovascular diseases (Lewis et al., 2011). Chronic diseases are responsible for disparities in physical impairment, hospitalizations and deaths among lower SES groups and racial/ethnic minorities. Chronic life strains due to lack of resources expose poor people to enormous psychological distress. As indicated earlier, education potentially confers coping skills to efficiently deal with daily psychological stressors (Pearlin, Menaghan, Lieberman, \& Mullan, 1981).

Several ways by which socioeconomic marginalization adversely affects physical health status and health care utilization patterns have been observed. Some studies (Stansfeld, Head, \& Marmot, 1997; Stansfeld, Smith, \& Marmot, 1993) show that minor psychiatric morbidities also play a mediating role between social circumstances and physical health status. Income or profit has a positive impact on individual's psychological well-being. Higher income is favorably related to several psychological indicators (Kaplan, Shema, \& Leite, 2008). Increase in income, access to better education and employment are tilted in favor of non-Hispanic Whites, however. Psychological 
well-being, in turn, contributes to regular physiological processes, thus, delays onset of chronic diseases that contribute to decline in physical health.

As demonstrated in some studies, socioeconomic status does not appear to completely explain race/ethnic difference in depressive symptoms (Skarupski et al., 2005) where baseline depressive symptoms among older individuals were higher among Blacks compared to Whites. Although adjusting for sex and SES variables reduced racial baseline difference in depressive symptoms, the remaining difference was still statistically significant. These racial differences increased overtime indicating differential distribution of depressive symptoms by SES and race over the life-course. Similar studies using the CES-D scale measure found that risk for depressive symptoms among Black and Hispanic women were higher compared to White and Asian women (Bromberger, Harlow, Avis, Kravitz, \& Cordal, 2004). Other constituents of the SES measure including wealth levels tend to protect individuals against depressive symptoms in the event of involuntary job loss among the elderly (Gallo et al., 2006) but such opportunities are limited for non-Hispanic Blacks and Hispanics due to their lower net-worth. Using lifecourse perspective, Miech and Shanahan (2000) showed that depressive symptoms diverged with age, where individuals with lower educational levels experienced higher symptoms. Lower education, income, wealth and occupational status are more common among racial/ethnic minorities, specifically among Blacks and Hispanics. Race/ethnic differences in health are reduced but not eliminated when SES variables are controlled, suggesting that discrimination or other psychological factors may play a role in contributing to these race/ethnic differences in health (Williams et al., 1997). 
The association between SES and depressive symptoms has also been established in homogenous samples from around the world. For instance, Lorant and colleagues (Lorant et al., 2007), in a Belgian Household Panel Survey data, found that longitudinal change in SES factors affected changes in depressive symptoms status. As one moves down the SES hierarchy, vulnerability to stressors and actual experience of stressors increases, and low SES individuals experience disproportionately frequent and extended emotional and psychological discomfort. The compounded effect of lower SES and racial/ethnic minority status results in persistent disparities in health status, health care utilization and mortality. These disparities in SES resources and the ensuing disproportionate burden of depressive symptoms lead to worse health outcomes for the poor and racial/ethnic minorities.

The present study used the Center for Epidemiologic Studies Depression (CES-D) scale as a mediator to examine the role of psychological factors in the pathways between SES and physical health status and overnight hospitalization by race/ethnicity.

\subsubsection{Behavioral Factors}

Health behaviors associated with morbidity and mortality include smoking (tobacco use), excessive alcohol consumption, sedentary lifestyle, diet and body mass index (BMI). Factors that determine BMI may vary but are believed to be related to diet, physical activity and genetics. These make it hard to use BMI as accurate measure of health behavior. Nonetheless, it has been used as proxy to some form of health behavior (Laaksonen, Prättälä, Helasoja, Uutela, \& Lahelma, 2003; Pampel, Krueger, \& Denney, 
2010; Wray, Alwin, McCammon, Manning, \& Best, 2006). Socioeconomic statuses are related to health behaviors (Laaksonen et al., 2003; Van Kippersluis \& Galama, 2013). Disproportionately high poverty levels, lower education, lower income and lower networth among minorities (Federal Interagency Forum on Aging-Related Statistics., 2012) means that these groups are highly unlikely to engage in protective health behaviors. The level of physical activity, for instance, depends on knowledge of the health benefits of regular physical activity and higher income allows access to resources related to physical activity. Some studies (Caspersen, Christenson, \& Pollard, 1986) found that physical activity was related to income and education, and others found that educational status predicted smoking status (Novotny, Warner, Kendrick, \& Remington, 1988; Pierce, Fiore, Novotny, Hatziandreu, \& Davis, 1989). Lower SES status was associated with poor diet intake and sedentary lifestyle (Krebs-Smith, Cook, Subar, Cleveland, \& Friday, 1995) because healthy food outlets and conditions for active lifestyle tend to be out of reach for lower SES individuals who happen to be racial/ethnic minorities. Further, lower SES individuals reside in areas that are not equipped with resources that encourage active lifestyle and healthy food outlets. Fast-food outlets tend to be dense in low income neighborhoods and interact with individual characteristics to explain obesity status $(\mathrm{Li}$, Harmer, Cardinal, Bosworth, \& Johnson-Shelton, 2009) which is related to chronic diseases. These health damaging behaviors increase as one moves down the SES hierarchy (Adler \& Conner-Snibbe, 2003).

In a longitudinal context, Lynch, Kaplan, and Salonen (1997) found that low SES men had poor dietary profile. This low SES-poor diet relationship is not limited to men, 
however. Poor neighborhoods also lack the necessary informational resources such as health promotion centers to change behavior. Other sources of information including schools and community centers in low income neighborhoods may lack sufficient resources or accessibility may be limited. On the other hand, SES is positively related to preventive health behaviors (Coburn \& Pope, 1974; Langlie, 1977). Higher SES individuals have the means to engage in preventive behavior avoiding costly hospitalizations with implications for their income and occupation. Therefore, apart from possessing the means, higher SES individuals have the incentive to maintain health which is expected to benefit them in the form of continued employment and lower out of pocket health care expenditures. Some studies hypothesized that sickness is costlier to the wealthy (van Kippersluis \& Galama, 2014), thus, the rich engage in health promoting behaviors such as smoking cessation and fewer risky behaviors such as binge drinking.

Poor dietary profiles could be a result of lack of healthy food outlets in the lower SES neighborhoods, lack of awareness of the benefits of healthy dietary habits, social influence, or a combination of any of these factors as described above. There is evidence for SES advantages or disadvantages cumulating across the life-course and influencing health outcomes later in life. In one study, poor adult health behaviors and psychosocial conditions were related to poor childhood environments, lower education and blue-collar employment (Lynch et al., 1997) indicating the harmful effects of prolonged lower SES status across the life-course. Although rates of poverty among Americans reduced, the reductions were not uniform across race/ethnic groups, where, non-Hispanic Blacks, Hispanics and other race/ethnic groups appear to have benefited the least. Comparison of 
median incomes and net-worth among different race/ethnic groups showed that more than a quarter of Hispanics and non-Hispanic Blacks still live in poverty. Further, some suggest that relative deprivation has adverse consequences on health (Sapolsky, 2004).

Another dimension in the SES-health behavior association by race/ethnicity pertains to attempts and successful quitting of health diminishing behaviors such as tobacco use, where, Whites and wealthier Americans have higher rates of successful quitting. Some studies examined the effects of SES on successful cessation of smoking. Although education and income were not associated with smoking onset, those who were more educated were more likely to attempt to quit smoking and those with high income were more likely to succeed in quitting (Winkleby, Cubbin, Ahn, \& Kraemer, 1999). These variations, then, increase the possibility of the onset of chronic diseases not only among those with lower education and lower income but also among those with higher education and lower income, creating and perpetuating SES based health disparities.

Research indicates associations between diseases, deaths and health diminishing behaviors. Some studies report that significant portion of premature mortality could be accounted for by behavioral factors, with tobacco use, patterns of diet and physical activity explaining most deaths (McGinnis \& Foege, 1993; Mokdad, Marks, Stroup, \& Gerberding, 2004). Such research designs do not take into consideration the macro-social environment that drives individuals to behave in a certain way and could potentially fail to identify appropriate policy levers or place the onus for better health status solely on the individual. 
A lower rank on SES is also associated with health damaging behaviors (Baum, Garofalo, \& Yali, 1999) because engaging in risk behaviors acts as an escape route from stressful situations, temporarily relieving the stress causing situation (Jackson et al., 2010). McLeod and Kessler (1990) found falling in low income category was consistently associated with exposure to adverse life events and lower SES persons lack resources to deal with these health-threatening events (National Research Council, 2004). Health behaviors have been consistently linked to an individual's SES and Hispanics and non-Hispanic Blacks consistently rank lower on SES.

Poverty among minorities, specifically among Native Americans, non-Hispanic Blacks and Hispanics is pervasive in America (Federal Interagency Forum on AgingRelated Statistics., 2012). Race/ethnic disparities in health behaviors are rooted in socioeconomic disparities such that Blacks and Hispanics show poorer health behaviors (National Research Council, 2004) because socioeconomic statuses limit available choices and opportunities. Relationships between SES variables and drinking may also vary by race/ethnicity. Less affluent Black men, for instance, consume higher levels of alcoholic beverage, similar to alcohol abuse (Jones-Webb, Hsiao, \& Hannan, 1995), but others (van Kippersluis \& Galama, 2014) observed that the rich drink more and smoke less.

\section{Summary}

Wealthier individuals with higher education and higher income and better job grade have lower morbidity and mortality. Higher SES individuals are presumed to build qualitatively better social relationships or social ties. Social relationships that provide 
emotional, appraisal, instrumental and informational support have also been shown to be associated with better health. Useful benefits may accrue from primary ties such as family or secondary ties such as volunteering in charitable organizations or belonging to social clubs. Social integration is embedded within the concepts of social relationships and social networks and SES is positively related to social integration. Psychological problems arising from stressors affect physiological processes. Absolute or relative poverty is related to chronic concern and feelings of lack of control which may unnecessarily stimulate the endocrine system, thus, generate chronic diseases such as diabetes, hypertension and heart disease. These are related to physical impairment and hospitalizations. Higher SES individuals are positioned to have better access to resources that can help mitigate the effects of stressors through better health behaviors.

Smoking, sedentary lifestyle, alcohol or drug abuse, lack of preventative care, and poor diet are some of the health diminishing behaviors. These behaviors are more common among lower SES individuals, in part because they act as escape routes from stressful environments. However, lack of affordability, for instance, time constraints to engage in physical activity, access to amenities for physical activity, access to healthful foods due to cost or neighborhood setting and lack of access to healthcare are some of the primary sources of barriers to healthy behaviors.

Ethnic and racial minorities in the United States fall in lower SES positions, thus, experience high morbidity and mortality rates. Socioeconomic status does not completely explain these disparities, however. Some studies point to everyday personal and 
institutional racism as another source for health disparities because Hispanics and Blacks at similar SES positions with Whites experience higher morbidity in many disease categories and have higher all-cause mortality. Absolute and relative poverty levels by race/ethnic group persisted over generations and the adverse disadvantages and effects may cumulate over one's life course.

Social relationships have been found to be positively associated with health behaviors, including tobacco use, diet, and exercise (Gottlieb \& Green, 1984). Health behaviors are also associated with psychological conditions (Lynch et al., 1997), and social relationships have been found to be associated with psychological factors (Glass, Leon, Bassuk, \& Berkman, 2006). Interaction among social, psychological and behavioral factors in predicting physical impairment status and overnight hospitalization are possible. The Reconstructability Analysis (RA) part of the analysis explored these potential interaction effects.

In summary, a large body of research established the inverse relationship between SES and morbidity, and between SES and mortality. Several studies have also reported relationships between SES and social integration, between SES and psychological factors, and between SES and health behaviors. Other studies provided evidence for the relationships between health behaviors and health status, between psychological factors and health status, as well as between social relationships and health status. Although the inverse relationship between SES and health status has been established, how SES, via social, psychological and behavioral factors may be related to health status is not 
sufficiently examined. This study examined, using a single survey data, the indirect relationships of each SES variable to physical health status and overnight hospitalization via three sets of mediators including social, psychological, and behavioral factors. 


\section{CHAPTER 3.0: PRESENT STUDY}

\subsection{Theoretical Background}

This study took an intersectional approach of two main theoretical orientations, namely cumulative dis/advantage theory and life course theory. Cumulative dis/advantage theory is traced to Price's essay on incidences of citations of scientific papers (cumulative advantages and disadvantages) in publications (Price, 1965) and Merton's “The Matthew Effect in Science” (Merton, 1968). The life course theory can be traced to (Cain, 1964) but was later advanced by Clausen, (1972) and Elder, (1974) among others.

Life-course theory refers to the several sequences of events an individual goes through in life and the roles that the individual assumes over the life course (Elder, 1998). Other approaches conceptualized life course theory as the study of the effects of exposure to health risks and subsequent impact on health outcomes through multiple pathways such as behavioral and psychosocial pathways across the life course (Ben-Shlomo \& Kuh, 2002). This theory points to the dynamic interplay between age, social structure and the role of the individual at various stages of his/her life guided by socio-cultural norms. Experiences, expectations and obligations at each stage over the life course vary by age and rely on stages of age. Independent of initial conditions, as described below, aging is related to becoming poor and onset of comorbidities, thus, like aging, becoming poor and onset of comorbidities are viewed as gradual processes. However, the course of these unavoidable consequences of aging may be affected by advantages or disadvantages 
originating at the beginning of life or even before conception (on parents' SES) and achieved level of SES in one's life.

Cumulative dis/advantage theory refers to the "systemic tendency for interindividual divergence in a given characteristic" (Dannefer, 2003, p. S327). Initial conditions are relevant to the cumulative dis/advantage theory because gains over time depend on initial comparative position in the social hierarchy. Merton later described cumulative advantage as the processes by which differences in initial advantages add incrementally increasing differences over time between those who have and those who lack resources (Merton, 1988). This theory can be used to examine systemic differences in the trajectory of health outcomes between individuals over time, owing to differences in initial SES conditions that continue to diverge over time. Cumulative dis/advantages of SES can be conceptualized to operate through social, psychological and behavioral pathways resulting in unfavorable or favorable health outcomes by race/ethnicity. Belonging to higher SES group as well as racial/ethnic majority provide better initial conditions which, over time, increase SES, affording psychosocial resources to withstand environmental hazards and mitigate risks to one's health. Disadvantages of unfavorable initial conditions for low SES groups and members of racial/ethnic minority cumulate over time increasing environmental stressors, unhealthy behaviors and social relationships that have negative impact on trajectories of health. These processes, over the life course, lead to systemically divergent trajectories in health outcomes between individuals at different levels in the SES hierarchy such that minorities and the poor experience worse health outcomes throughout their lives. 
Guided by the above theoretical perspectives, this dissertation investigated the process by which SES variables were related to social, psychological and behavioral factors, and in turn, the relationship of each of the proposed mediating variables to physical impairment status and overnight hospitalizations, and whether these pathways varied by race/ethnicity. The next section further elaborates the objectives and conceptual models that motivated this study. Simple conceptual models of the proposed pathways are provided schematically in Figure 2 for the SEM approach and in Figures 3 and 4 for the RA approach.

\subsection{Conceptual Model and Objectives of the Study}

This study had two general objectives. The first general objective was to examine whether the trajectory of social, psychological and behavioral variables as mediators in the relationship between SES and physical health status and overnight hospitalization differed by race/ethnicity. Positing that SES was indirectly related to physical health status and overnight hospitalization, this study used Structural Equation Modeling (SEM) approach to examine the mediating role of social, psychological and behavioral factors in the SES-physical impairment, and SES-overnight hospitalization links. Racial/ethnic disparities in physical health status and overnight hospitalization, therefore, were expected to result from differences in the trajectories of the proposed mediating variables

due to differences in SES, and the differences in mediating variables, in turn, were related to the trajectories of physical impairment and overnight hospitalization. A simple schematic of the conceptual path models is provided below (Figure 2). 


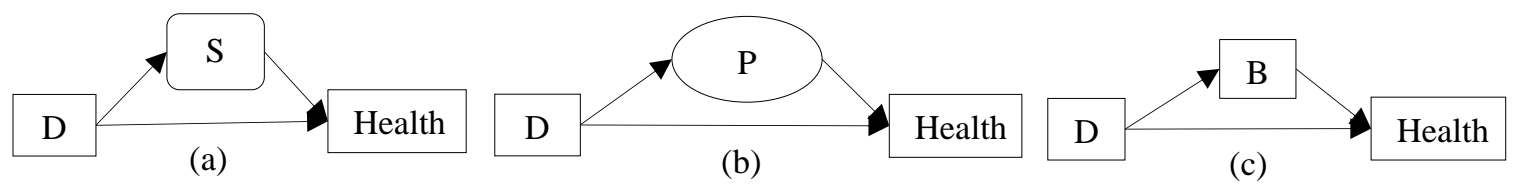

Figure 2: Conceptual models for SEM analysis

Note: $\mathrm{D}=$ Socioeconomic status/demographic, $\mathrm{P}=$ latent depressive symptoms, $\mathrm{B}=$ behavioral (observed) variables, $\mathrm{S}=$ social integration (index), and Health=health outcome variables.

The second general objective, using exploratory modeling techniques, namely Reconstructability Analysis (RA) approaches, was multi-pronged. First, it used SES variables (i.e., income, wealth, education and employment status), social, psychological, and behavioral variables concurrently to predict physical impairment status and overnight hospitalization with the aim of identifying predictive variables, interaction effects of the predictive variables and differences in the selected predictive variables by race/ethnicity without examining indirect relationships (Figure 3). This allowed for selection and investigation of SES and social, psychological and behavioral $\{$ SPB $\}$ variables predictive of physical impairment and overnight hospitalization (Note that in Figure 3, $\mathrm{D}=\mathrm{SES} /$ demographic [i.e., income, wealth, education, employment status, gender and age], $\mathrm{S}=$ social, $\mathrm{P}=$ psychological, $\mathrm{B}=$ behavioral and represent several variables) and the interaction effects of the predictive variables as has been suggested in previous studies (Adler \& Rehkopf, 2008; Adler et al., 1994). Throughout the document, the social, psychological and behavioral factors are represented by $\{\mathbf{S P B}\}$ for convenience. Note that for the RA analysis, several social, psychological, and behavioral variables constituted $\mathbf{S}, \mathbf{P}$, and $\mathbf{B}$ factors in that order. In SEM analysis, the psychological variable (P) was a latent variable, and the social integration variable (S) was an index, while smoking, levels of alcoholic beverage consumption, physical activity and body mass 
index (BMI) constituted behavioral factors (B). Reconstructability Analysis, therefore, investigated relationships between single item independent variables (IVs) and the outcome variable and compared differences in predictive IVs and examined composite IV states that increased/reduced risk of physical impairment or overnight hospitalization across race/ethnic groups.

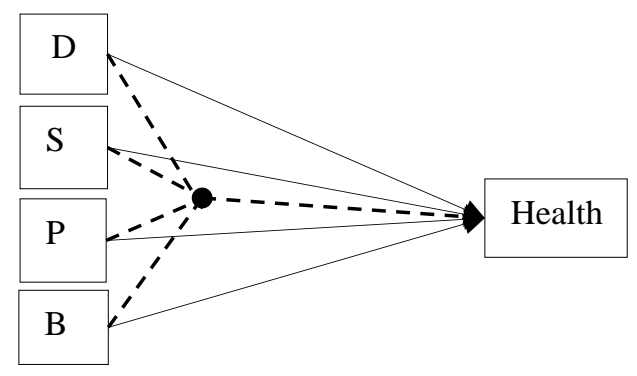

Figure 3: Conceptual model for RA

Note: The dashed lines indicate that model search also allowed for any interaction effects among variables. $\mathrm{D}=\mathrm{SES} /$ demographic, $\mathrm{S}=$ Social, $\mathrm{P}=\mathrm{Psychological}, \mathrm{B}=$ Behavioral, Health=Health outcome variables

In path analysis using the RA approach, psychological, behavioral and social factors most closely related to SES were identified and used in the indirect path between SES and health variables (Figure 4). Strengths of path values from selected SES/demographic to selected $\{$ SPB $\}$ and from selected $\{$ SPB $\}$ to physical impairment and overnight hospitalization, were compared by race/ethnicity when selected SES/demographic variables and selected $\{\mathbf{S P B}\}$ variables for race/ethnic groups were identical as illustrated below (Figure 4). In RA, five social (S), three psychological (P), and four behavioral (B) variables were used instead of the integrated respective social integration index and latent psychological variables used in the SEM section of the dissertation. RA easily handles binary and multi-category nominal variables. While integrated variables making an index provide a higher level view of relationships, RA 
provides a fine grained view of differences or similarities in relationships between the independent variables, the intermediate variables, and the outcome variables (comprehensive descriptions of variables are provided in Chapter 4 - Methods).

In Figure $4, \mathrm{D}_{i} \rightarrow S_{j}$ represents quantifying the effect of the selected D variable on the selected $S_{j}$, whereas $\mathrm{P} \rightarrow \mathrm{PI} \mid \mathrm{D}_{i}$ quantifies the effect of the selected $S_{j}$ variable on PI controlling for $\mathrm{D}_{i}$, and $\mathrm{D}_{i} \rightarrow \mathrm{PI} \mid S_{j}$ indicates quantifying the effect of $\mathrm{D}_{i}$ on PI controlling for the effect of $\mathrm{S}_{j}$. Note that $\mathrm{D}_{i}$ may be one or more variables but $S_{j}$ and PI represent single variables. For psychological and behavioral factors, substitute $P_{j}$ and $B_{j}$ variables respectively.

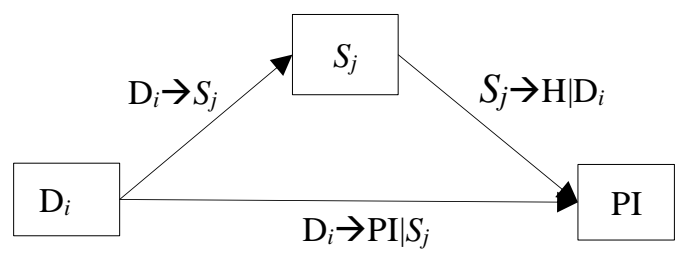

Figure 4: Conceptual model with selected variables in RA path analysis

Note: $\mathrm{D}_{i}=$ Selected socioeconomic/demographic variable, $\mathrm{S}_{j}=$ selected social variable and $\mathrm{PI}=$ physical impairment (\& overnight hospitalization).

\subsection{Research Questions}

The specific research questions for the first part (SEM) of the analytic approach were:

1. Does the effect of SES on:

i. Social factors vary by race/ethnicity?

ii. Psychological factors vary by race/ethnicity?

iii. Behavioral factors vary by race/ethnicity?

2. Do social, psychological and behavioral factors mediate the effects of SES on:

i. Physical impairment status?

ii. Overnight hospitalization? 
3. Do the indirect effects of SES on:

i. Physical impairment via social, psychological and behavioral factors vary by race/ethnicity?

ii. Overnight hospitalization via social, psychological and behavioral factors vary by race/ethnicity?

The specific research questions for the RA part of the analytic approach were:

1. Do SES/demographic, social, psychological and behavioral variables predict:

i. Physical impairment status and do these predictive variables vary by race/ethnicity?

ii. Overnight hospitalizations, and do these predictive variables vary by race/ethnicity?

2. Do SES/demographic variables associated with social, psychological, and behavioral variables vary by race/ethnicity?

3a. Do path values from SES/demographic to social, psychological and behavioral variables vary by race/ethnicity?

3b. Do path values from social, psychological and behavioral variables to:

i. Physical impairment status vary by race/ethnicity?

ii. Overnight hospitalizations vary by race/ethnicity? 


\section{CHAPTER 4.0: METHODS}

\subsection{Data Source, Procedure and Sample}

This study used the Health and Retirement Study (HRS) data, a nationally representative multi-stage area probability sample that biennially surveys individuals over the age of 50 and their spouses. The study is designed to collect extensive information on how older Americans cope with rapidly changing living arrangements including retirement, health insurance, income, employment, social relationships, physical health status, health behaviors, psychological status and health care utilization among other factors.

The target population for the original HRS study was noninstitutionalized adults in the contiguous United States born between the years 1931 and 1941 (Heeringa \& Connor, 1995; Institute for Social Research, University of Michigan, 2016; Wallace \& Herzog, 1995). The unit of observation was an eligible household financial unit. The HRS used a multi-stage area probability design with four distinct selection stages including probability proportionate to size (PPS), selection of Metropolitan Statistical Areas (MSAs), and non-MSA counties. This was followed by a second stage sampling

area segments (SSUs) within sampled primary stage units (PSUs). Listing of all housing units (HU) was made and in the third sampling stage, a systematic selection of housing units from the HU listing was made. Finally, an age eligible person within HU sample was selected. Residents of the state of Florida, Blacks, and Hispanics were oversampled to improve representation of minority populations. Details of the HRS study are provided in the University of Michigan's Institutional Social Research website and in other reports 
(Heeringa \& Connor, 1995; Institute for Social Research, University of Michigan, 2016; Wallace \& Herzog, 1995).

The HRS began in 1992 with a nationally representative sample of 12,654 men and women between the ages of 51 and 61 and their spouses in 7700 households. Sponsored by the National Institute on Aging (NIA), the HRS continues to administer follow up surveys every two years. Currently, the HRS comprises of seven cohorts: the original HRS cohort (born 1931-1941), the AHEAD cohort (born before 1923), Children of the Depression (CODA) cohort (born 1924-1930), War Baby (WB) cohort (born 19421947), Early Baby Boomer (EBB) cohort (born 1948-1953), Mid-Baby Boomer cohort (born 1954-1959) and Late Baby Boomer (LBB) cohort (born 1960-1965).

The 1992 HRS collected data from households with at least one age-eligible respondent and the spouse regardless of the spouse's age eligibility. Therefore, the original HRS cohort included spouses of respondents who were not born between 1931 and 1941. These not-age-eligible spouses of age eligible respondents are not a random representative sample of their age cohort, and thus, analysis of the data including the age ineligible respondents can lead to biases (Jenkins, Ofstedal, \& Weir, 2008) thus were excluded from this study.

Focusing only on the original HRS cohort, this study used data collected between 2002 and 2010 (5 waves) for the Structural Equation Modeling part and data collected between 2004 and 2008 (3 waves) for the Reconstructability Analysis part of the study. 


\subsection{Measures}

There were three variable categories in this study: independent variables (IVs), mediating variables, and dependent variables (DVs). Differences in variable construction and transformation for either the SEM or RA part of the analyses are noted in the sections below.

\subsubsection{Independent Variables}

Socioeconomic status (SES), represented by income, education, occupation and wealth, has been used as a proxy for social status location in the society. This dissertation used total household income, total wealth minus debt, employment status, and education level as separate (i.e., without making an SES index) independent variables representing SES.

Income. Total household income was computed by summing all income including income from regular employment, annuities, Social Security benefits, income from unemployment or workers compensation and any other government income for the respondents and their spouses.

In the RA part of the analysis, income was binned to three categories representing low, medium and high, using equal sample binning program in the SPSS program (IBM Corp., 2012). The ranges of categories for income were as follows: less than $\$ 26,000$ coded $0, \$ 26,000$ to $\$ 54,000$ coded 1 , and greater than $\$ 54,000$ coded 2 , as low, medium and high respectively. 
Wealth. The HRS asked respondents several questions about wealth and debt. Total wealth was computed by summing the wealth components including businesses, individual retirement accounts, stocks and bonds, pensions, checking and savings accounts, and primary residence. The income and wealth variables were transformed using natural log to adjust for skewness and extreme ranges (Gelman \& Hill, 2007).

In the RA part of the analysis, wealth was binned to three categories representing low, medium and high, using equal sample binning program in the SPSS program (IBM Corp., 2012). The ranges for wealth categories were: less than $\$ 100,000$ coded 0 , $\$ 100,000$ to $\$ 350,000$ coded 2, and over $\$ 350,000$ coded 3, as low, medium and high respectively.

Education. Education was measured by the number of years of formal schooling ranging from 0 for no formal schooling to 17 for higher (for college graduate and beyond) education. The HRS asked respondents, "What is the highest grade of school or year of college you completed?" with integer response options ranging from 0-17.

In the RA method, education was binned to three categories, indicating less than high school diploma [0-11] coded 0 , high school diploma [equal to grade 12] coded 1 , college and over [13-17]) coded 2.

Employment status. Employment status was a binary variable where 0 indicated unemployment and 1 indicated employment. The HRS asked respondents "Are you doing 
any work for pay at the present time?" with response options 'no' coded 0 , and 'yes' coded 1 in this study.

In the RA method, a different question with several response options was used because analysis of multi-category data in OCCAM is handled easily and in RA (implemented in OCCAM) can detect interaction effects for nominal variables with more than two categories. The HRS asked respondents, "Are you working now, temporarily laid off, unemployed and looking for work, disabled and unable to work, retired, a homemaker, or what?" This variable was collapsed to three categories including 'employed', 'retired', and 'other' coded 1, 2, 3 respectively. The 'other' category included the temporarily laid off, unemployed and looking for work, disabled or unable to work and homemaker categories.

Lagged path models and latent growth curve models adjusted for demographic factors including age and gender, where gender was coded 0 for males and 1 for females. Age was a continuous variable in the SEM part of the analysis but was binned to three categories in RA (63-65, coded 0; 66-69, coded 1; and 70-73, coded 2). Note that for the RA part of analysis data collected between 2004 and 2008 was used. 
Table 1: Summary of variables used for statistical mediation analysis and RA method

\begin{tabular}{|c|c|c|c|c|}
\hline & \multicolumn{2}{|c|}{ Statistical Mediation } & \multicolumn{2}{|c|}{$\begin{array}{c}\text { Reconstructability } \\
\text { Analysis }\end{array}$} \\
\hline Variables & Variable type & $\begin{array}{l}\text { Range or Mean } \\
\text { (baseline) }\end{array}$ & $\begin{array}{l}\text { Variable } \\
\text { type }\end{array}$ & Cardinality \\
\hline \multicolumn{5}{|l|}{ SES/Demographic } \\
\hline Income (logged) & Continuous & $0-7,395,294$ & Nominal & $0,1,2$ \\
\hline Wealth (logged) & Continuous & $\begin{array}{l}-480864.79- \\
41,140,000 \\
\end{array}$ & Nominal & $0,1,2$ \\
\hline Education & Continuous & $0-17$ & Nominal & $0,1,2$ \\
\hline Employment & Binary & 0,1 & Nominal & $1,2,3$ \\
\hline Gender & Binary & 0,1 & Binary & 0,1 \\
\hline Age & Continuous & $61-71$ & Nominal & $0,1,2$ \\
\hline Psychological & & & & \\
\hline Positive affect & \multirow{3}{*}{ Latent variable } & $0, .5,1$ & \multirow{3}{*}{ Binary } & $0,1(1-2)$ \\
\hline Negative affect & & $0, .333, .666,1$ & & $0,1(1-3)$ \\
\hline Somatic symptoms & & $0, .333, .666,1$ & & $0,1(1-3)$ \\
\hline \multicolumn{5}{|l|}{ Social } \\
\hline Friends near & \multirow{5}{*}{ Index } & \multirow{5}{*}{$0-5$} & Binary & 0,1 \\
\hline Relatives near & & & Binary & 0,1 \\
\hline Frequency of social contact & & & Nominal & $0,1,2$ \\
\hline Marital status & & & Nominal & $1,2,3,4$ \\
\hline Volunteering in orgs. & & & Binary & 0,1 \\
\hline \multicolumn{5}{|l|}{ Behavioral } \\
\hline BMI & Continuous & $12.2-64.4$ & Binary & 1,2 \\
\hline Drinking & Continuous & $0-15$ & Nominal & $0,1,2$ \\
\hline Exercise & -- & -- & Nominal & $0,1,2$ \\
\hline Smoking & -- & -- & Nominal & $0,1,2$ \\
\hline \multicolumn{5}{|l|}{ Outcome variables } \\
\hline Physical Impairment (ADL) & Index & $0-6$ & Binary & $0,1(1-6)$ \\
\hline Overnight hospitalization & Binary & 0,1 & Binary & 0,1 \\
\hline
\end{tabular}

Note: In the last column, 1(1-2), 1(1-6)... indicate that 1-2 and 1-6 categories were collapsed across levels. 


\subsubsection{Mediating Variables}

Mediating variables (or intervening variables), are variables that transmit the effects of the predictor to the dependent variable. This study used social, psychological, and behavioral variables as mediating variables with the objective of investigating the effects of SES/demographic factors on the trajectory of these mediating variables and the role that change in the mediating variables played in the trajectory of physical impairment and overnight hospitalization.

\section{Social Factors}

A modified social integration index, based on Berkman \& Syme's, (1979) social network index (SNI) and closely resembling two other similar indices of social integration used recently (Ertel, Glymour, \& Berkman, 2008; Yang, Li, \& Ji, 2013), this study used the five binary social variables described below to construct a social integration index that ranged from $0-5$, where, higher scores indicated greater integration. In the RA part of the analysis, each of the five variables was used as a stand-alone social variable, where, marital status and frequency of social contact variables were multicategory (Table 1) and all other social variables were binary. Each of the survey questions is described below.

Friends in the neighborhood. The HRS asked respondents, "Do you have any good friends in or near the facility?/Do you have any good friends living in your neighborhood?", to establish if respondents had good friends in their neighborhoods that 
may serve as social contacts. The response options for this question were 'yes' and 'no' and were coded 1 for a 'yes' response and 0 for a 'no' response.

Relatives in the neighborhood. To establish whether respondents had relatives living in their neighborhoods, the HRS asked respondents, "Do you have any relatives in or near the facility where you are living?/Besides the people living here with you, Do you have any relatives in your neighborhood?". The response options for this question were 'yes' and 'no' and were coded 1 for a "yes" response and 0 for a "no" response in this study.

Frequency of contact with other people. To further assess social integration, the HRS asked the number of times respondents met with people, "How often do you get together with [people in or near the facility/any of your neighbors] just to chat or for a social visit?", and a follow up question to identify the number of times respondents get together with people 'per day', 'per week', 'per two weeks', 'per month', 'per year' and 'almost never'.

Using these two questions, a continuous indicator was computed to measure the frequency of meeting with people per week ranging from 0-7. For the SEM part of the analysis, this variable was dichotomized to indicate respondents getting together 'less than once a week' coded 0 and 'at least once a week' coded 1.

For the RA part of the analysis, this variable was binned to indicate low integration (including those who respond 'almost never') coded 0 , medium integration 
(including those who met once a week to three times a week) coded 1, and high integration (which included those who met four times a week or more) coded 2.

Marital status. In the first part of the analysis (i.e., SEM), a binary variable indicating 'coupled/married' coded 1 , and 'not coupled/not married' coded 0 , was used.

In the RA part of the analysis, marital status had four categories including 'married/partnered' coded 1, 'separated and/or divorced' coded 2, 'widowed' coded 3, and 'never married' coded 4 . The four-category marital status variable used in RA was constructed from four separate questions in the HRS and this variable was included the public used data sets.

Volunteering in organizations. Volunteering in organizations provides an opportunity to form connections that enhance social interactions and has been used as one of the measures of social integration. The HRS asked respondents 'Have you spent any time in the past 12 months doing volunteer work for religious, educational, health-related or other charitable organizations?' with a 'yes' and 'no' response options. A 'no' response was coded 0 and a 'yes' response was coded 1.

\section{Psychological Factors}

Several instruments are used to measure depression and depressive symptoms. These include, The Beck Depression Inventory (BDI), Center for Epidemiological Studies-Depression Scale (CES-D), Geriatric Depression Scale (GDS), Hospital Anxiety and Depression Scale (HADS), and Primary Care Evaluation of Mental Disorders-Mood 
Module (Prime-MD). The short form of the CES-D Scale measure adopted by the HRS was used in this study.

Material conditions and SES specifically are linked to psychological status. The Center for Epidemiologic Studies Depression Scale (CES-D Scale) (Radloff, 1977) is a measure of depressive symptoms that has been used in several studies. The HRS used a modified short version of the CES-D Scale to collect data on negative affect factors, somatic factors and positive affect factors (reverse coded). The HRS included 11-item CES-D scale questions in 1992 instead of the 20 original CES-D scale questions, and only 8 CES-D scale questions in subsequent waves. Question format and response options also changed between 1992 and 1994 waves where the format for the year 1994 and later waves were binary and remained consistent thereafter. The HRS asked respondents, "Now think about the past week and the feelings you have experienced. Please tell me if each of the following was true for you much of the time this past week. Much of the time during the past week, you... Would you say yes or no?" followed by 8 questions such as 'you felt depressed' and 'you felt activities were efforts' in place of the '...' This research used data from 2002 to 2010 waves, thus, the 8 -item short form was used as an indicator of depressive symptoms. Questions in the positive direction "you felt happy within the previous two weeks" and "you enjoyed life within previous two weeks" were reverse coded. The somatic symptoms sub-scale was composed of three binary questions, the positive affect factor by two binary questions, and the negative affect factor by three binary questions. The CES-D scale is a three factor scale, thus, in the SES 
part of the study, a latent variable was used to represent psychological factors (Shafer, 2006).

In the RA part of the analysis, the three sub-scales, namely somatic symptoms, negative affect and positive affect (reverse coded) were used as stand-alone psychological variables with 0 indicating absence and 1 indicating presence of depressive symptoms.

Somatic symptoms. Three binary questions regarding restless sleep, everything being an effort and not being able to get going were used to create a dichotomous variable with 1 indicating any 'yes' response to any of the three questions and 0 coding for 'no' response to all three questions.

Negative affect. Three binary questions about feeling sad, feeling depressed and feeling lonely were used to create a binary variable with 1 for any 'yes' to any of the questions and 0 indicating a 'no' response to all three questions.

Positive affect. Two binary questions pertaining to feeling happy and enjoying life in the previous week were used to create a binary variable reverse coded to indicate 1 coding for any 'no' and 0 coding for 'yes' response to both questions. Therefore, a code of 1 represents absence of positive affect and 0 represents presence of positive affect.

\section{Behavioral Factors}

Health relevant behavioral factors associated with morbidity and mortality include cigarette smoking (tobacco use), excessive alcoholic beverage consumption (drinking), 
lack of physical activity (exercise) and lack of appropriate diet among others. Four behavioral factors were considered in this study. The HRS collects extensive data on tobacco use, physical activity, alcohol use and change in respondent weight over time as described below.

Cigarette smoking. In 1992, the HRS asked respondents, "Have you ever smoked cigarettes?" Regarding current smoker status, the HRS asked respondents, "Do you smoke cigarettes now?" with a 'yes' or 'no' response options. This question format was maintained in all subsequent waves. In computing smoking status, current smoker status was conditioned on, "Have you ever smoked cigarettes?" question that was asked in the first wave (1992). The cigarette smoking variable was highly correlated overtime and was dropped from the SEM analysis in this study.

In the RA part of the analysis, this variable was categorized into 'never smoker' coded 0 , 'former smoker' coded 1 and 'current smoker' coded 2.

Alcoholic beverage consumption (Drinking). Several questions were used to obtain information on alcoholic beverage use. In this study, two questions were used to compute average level of alcohol consumption per week. The HRS asked respondents, "In the last three months, on average, how many days per week have you had any alcohol to drink? (For example, beer, wine, or any drink containing liquor.)", and "In the last three months, on the days you drink, about how many drinks do you have?" A continuous variable, indicating average number of drinks per week per person was computed using these two variables. 
In the RA part of the analysis, the continuous variable was collapsed to three categories including 'less than moderate drinking', coded 0, 'moderate drinking', coded 1, and 'excessive drinking', coded 2.

Physical activity. To collect information on respondent's physical activity, the HRS asked respondents, "How often do you take part in sports or activities that are vigorous, such as running or jogging, swimming, cycling, aerobics or gym workout, tennis, or digging with a spade or shovel: more than once a week, once a week, one to three times a month, or hardly ever or never?', with response options, 'never', 'some of the time' and 'a lot of the time'. However, from 2004 onwards, the HRS altered the wording of the physical activity question and frequency distribution of responses before and after change in the wording appeared to differ. Because the wording changed from 2002 to 2004, and the SEM analysis part of the study used data from 2000-2010, the variable was dropped in the SEM part of the study.

The RA analysis used data from 2004-2008 where this variable was consistently asked without altering question format, and thus, was included in the analysis where it was binned to three categories indicating 'no exercise' coded 0 , 'some exercise' coded 1 and 'frequent exercise' coded 2.

Body mass index. Body mass index (BMI), although controversial, has been used as an indicator of healthy eating habits and physical activity. Body mass index was computed from the 1992 height measures and respective weights measured at successive data collection time points. In 1992, the HRS asked respondents, "How tall are you?" in 
feet and "How tall are you?" in inches. The aim of these questions was to establish height in feet and inches (e.g., 5 feet and 8 inches). To obtain information about weights over time the HRS asked respondents, "About how much do you weigh?" and responses were provided in pounds. In BMI calculation, respondent weight in kilograms is divided by respondent height in centimeters squared (i.e., (weight/[height $\left.]^{2}\right)$ ).

In the RA part of the analysis, BMI was binned to two categories as 'normal weight' coded 1 and 'overweight/obese' coded 2.

\subsubsection{Dependent Variables}

The outcome variables used in this study were physical impairment status which was measured by activities of daily living (ADL) and overnight hospitalization.

\section{Physical Impairment}

In six separate questions, the HRS asked respondents, "Because of a health or memory problem, do you have any difficulty with...?" followed by the specific questions: "dressing, including putting on shoes and socks?"; "walking across a room?"; "bathing or showering?"; "eating, such as cutting up your food?"; "getting in or out of bed?"; and "using the toilet, including getting up and down?". A total score of physical impairment index was calculated by summing the individual item responses forming an index ranging from 0 to 6 , where higher scores indicated higher difficulties with physical functioning, that is, higher levels of physical impairment. 
In the RA part of the analysis, the physical impairment variable was converted to two categories to indicate 'no physical impairment in any functional area' coded 0 and 'at least one physical impairment indicator' coded 1. A response of 'yes' to at least one of the questions, thus, was coded 1 in the RA analysis.

\section{Overnight Hospitalization}

Overnight hospitalization was a binary variable assessed by a single question. The HRS asked respondents, "In the last two years (since previous wave), have you been a patient in a hospital overnight?' with a 'yes' and 'no' response options. A 'yes' response was coded 1 and a 'no' response was coded 0 in this study.

\subsection{Analytic Approaches}

Two analytic approaches were used to evaluate the proposed models. The first analytic approach that was used in the first part of the dissertation was mediation analysis using Structural Equation Modeling (SEM) and the second approach was Reconstructability Analysis (RA).

In the first approach, the aim of the study was to examine variations of the effects of each of the SES variables on physical health status and on overnight hospitalization, through psychological, behavioral and social integration variables by race/ethnic group using lagged path analysis and latent growth curve analysis. Predictive paths from SES to the mediators and from the mediators to the outcome variables, among the three race/ethnic groups, were compared using multiple group analysis approach. Lagged path analysis provides information on the stability of rank order of subjects on various 
variables but does not provide information regarding the trajectories of inter-individual or intra-individual change over time. In order to model inter-individual (variation between individuals) and intra-individual (variations of an individual's score over time) trajectories over time, latent growth curve modeling was used to investigate the effects of SES on the growth factor for physical impairment status through the growth factor for social integration and through the growth factor for depressive symptoms.

In the second analytic approach, using RA, this dissertation explored the direct effects of SES, social, psychological, and behavioral factors on physical impairment status and on overnight hospitalization. Further, SES to $\{$ SPB $\}$, and $\{$ SPB $\}$ to physical impairment status and overnight hospitalization paths were examined for SES variables that were predictive of $\{$ SPB $\}$ variables. Path values from selected SES to selected social, psychological, and behavioral variables as well as from selected $\{\mathbf{S P B}\}$ to physical impairment and overnight hospitalization were computed and compared by race/ethnicity. As a reminder, $\mathbf{S}$ constituted 5 variables, $\mathbf{P}$ constituted 3 variables, and $\mathbf{B}$ constituted 4 variables.

\section{Rationale for Analytic Approaches}

Grounded on specific hypotheses, mediation analysis allows for modeling indirect relationships between predictor and outcome variables through a mediating variable. Associations are hypothesized a priori and modeling is conducted to find in favor of or against the hypothesized relations. The objective in the first part of this study was to investigate hypothesized differences in how SES variables predicted the outcome 
variables through the mediating variables. Specifically, the primary interest of the study was to examine the role of the proposed mediating variables in the indirect association between SES variables and physical impairment, and between SES and overnight hospitalization. This method also allowed the use of latent variable modeling techniques to examine trajectories of growth factors, computation of effect sizes, confidence intervals and testing these effect sizes for significance by race/ethnic group.

Reconstructability Analysis uses information and graph theory techniques in modeling data and has several modeling options. Of these several options, this study first used a directed system modeling where one variable (physical impairment or overnight hospitalization) was set as a dependent variable (DV) and all SES/demographic, social, psychological, and behavioral variables were set as independent variables (IVs). The algorithm, then, selected any single and/or joint predictors out of the several SES/demographic, social, psychological, behavioral variables, thus, providing a simpler representation of the structural relationships in the data. Strengths of association, using uncertainty reduction in the DV, were also computed to indicate information captured. Therefore, the RA approach can be viewed as exploratory modeling that can reveal interaction effects, single predictors and level of uncertainty reduction in the DV by the predictive IVs. Secondly, this study used an RA method that identified associations among variables using neutral system modeling, that is, without making distinction between IV and DV variables. This process identified, for instance, SES variables that were strongly associated with social, psychological, and behavioral variables, and these 
associated social, psychological, and behavioral variables were then used in further directed path modeling.

Thus, mediation analysis and Reconstructability Analysis were used as complementary analytic approaches in this study, where, the first method (SEM) was used primarily in a confirmatory mode that assumed linear relationships between variables and the second method (RA) was primarily used in exploratory mode to select most informative predictors and compute uncertainty reduction in the DV due to the selected predictors. Both approaches were used to examine the role of the mediating variables in the association between SES variables and health variables by race/ethnicity.

\subsubsection{Statistical Mediation Analysis}

Statistical mediation can be defined as the causal process or mechanism by which an independent variable affects a mediator, and the mediator, in turn, affects the outcome variable (Cole \& Maxwell, 2003; MacKinnon, 2008). In mediation analyses, magnitudes of association as well as direction (i.e., whether two quantities increase or decrease together, or one decreases while another increases) are obtained. This method is suitable for testing hypotheses by computing direct and indirect effect sizes, standard errors, and by conducting significance tests. The present study used multiple group lagged path analysis with observed variables and multiple group analysis in a growth curve modeling framework to examine differences in path coefficients by race/ethnicity. The lagged regression models used data from three time points. Analyses were conducted using 
Mplus 7.4 (Muthén \& Muthén, 1998-2015), SPSS 21.0 (IBM Corp., 2012) and Stata 13 (StataCorp., 2013).

Preliminary analyses to ascertain univariate and multivariate normality of distribution of scores were conducted using SPSS 21. Distributions whose univariate skewness approaching the value of 2 and univariate kurtosis approaching the value of 7 generally present challenges in maximum likelihood (ML) estimation based results (Finney \& DiStefano, 2006). Descriptive statistics for the independent variables, mediators and dependent variables were computed to examine growth patterns and bivariate relationships. Longitudinal mediation analysis using Structural Equation Modeling (SEM) was conducted.

\section{Mediation process}

Following Cheong and colleagues (Cheong, MacKinnon, \& Khoo, 2003), a mediation process was defined as the change in independent variables, influencing the trajectory in the mediating variables, and change in the mediating variables, in turn, predicting change in the outcome variables. In the $X \rightarrow Y$ relationship (Figure 5, below), for instance, $\tau$ represents the total effect of $X$ on $Y$ (i.e., $\tau$ represents the unstandardized slope of the regression of $Y$ on $X$ ). When the mediator, $M$, is introduced (Figure 6), $\tau^{\prime}$ represents the direct effect of $X$ on $Y$ after controlling for the effects of the mediator, $M$, on Y. Thus, $\alpha$ represents the effect of $X$ on $M$ and $\beta$ represents the effect of $M$ on $Y$, controlling for the effect of $X$ on $Y$. The indirect effect is the product of $\alpha$ and $\beta$ (i.e., $\alpha \beta$ ) paths. Indirect effect is the effect that the independent variable (X) has on the dependent variable $(\mathrm{Y})$ through the mediator $(\mathrm{M})$. For a multiple mediator model, the indirect effect 
is calculated as the sum of products of the indirect paths as described (Figure 7, below). $\mathrm{X}, \mathrm{M}$ and $\mathrm{Y}$ are used for convenience but they correspond to SES/demographic variables, $\{$ SPB $\}$ variables and health variables $(\mathrm{PI}$ or $\mathrm{OH})$ respectively.

The equation for the relationship in Figure 5 below is given by,

$\mathrm{Y}_{3}=$ Intercept $_{1}+\tau \mathrm{X}_{1}+\varepsilon_{1}$ where $\tau$ is the regression coefficient and, $\varepsilon_{1}$ is error term which assumed to be identically and independently distributed (i.i.d.).

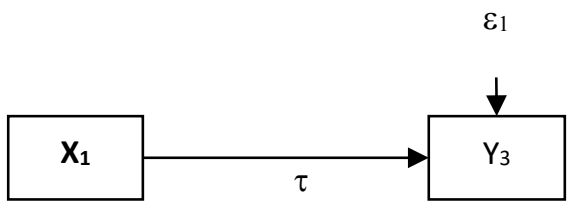

Figure 5: Total effects of $\mathbf{X}_{1}$ (SES variables) on $\mathrm{Y}_{3}$ (outcome variable at the third time point).

Upon introducing a mediator, the basic path diagram illustrating mediation model is provided (Figure 6) below followed by the associated regression equations.

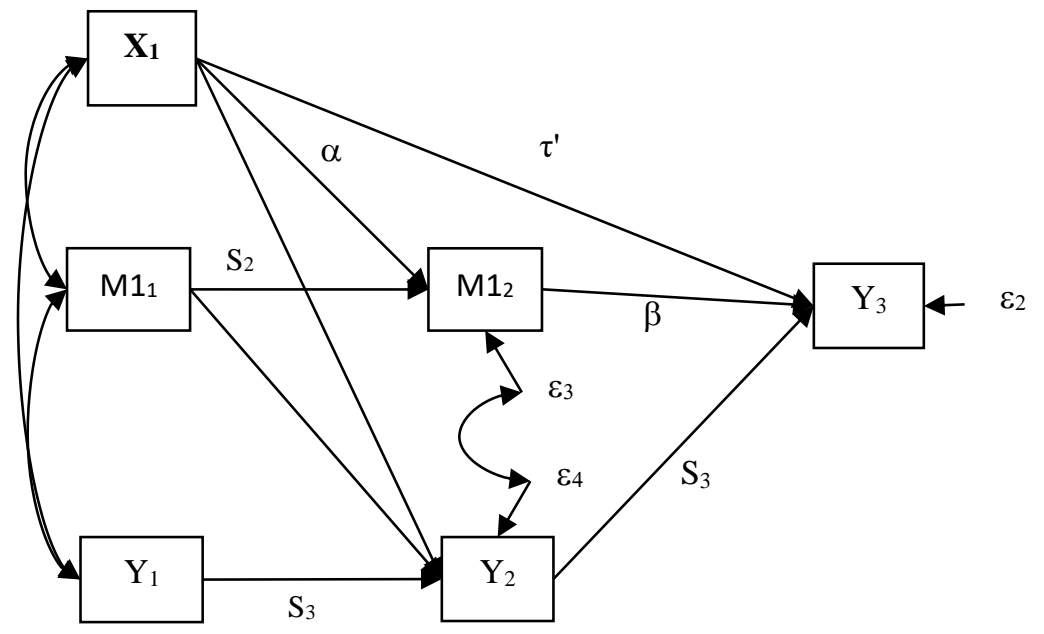

Figure 6: Path diagram and mediation model for a single mediator model, $\mathrm{t}=$ time.

Note: Two headed curly arrows indicate covariance. All regression paths are not shown for simplicity. 
Equations for the mediator and outcome variables ( $\mathrm{M}_{2}$ and $\mathrm{Y}_{3}$ respectively) in Figure 6 are given by: $\mathrm{M}_{2}=$ Intercept $_{2}+\alpha \mathrm{X}_{1}+\mathrm{s}_{2} \mathrm{M}_{1}+\varepsilon_{3} \quad$ (effect of $\mathrm{X}_{1}$ on $M 1_{2}$ adjusted for prior effect of $\mathrm{M}_{1}$ ) $\mathrm{Y}_{3}=$ Intercept $_{3}+\tau^{\prime} \mathrm{X}_{1}+\beta M 1_{2}+\mathrm{s}_{3} \mathrm{Y}_{2}+\varepsilon_{2} \quad$ (adjusted effects of $M 1_{2}$ and $X_{1}$ on $\mathrm{Y}_{3}$ )

The above single-mediator model is easily extended to multiple mediator models as shown below (Figure 7). These path models investigated lagged effects, with the outcome controlled for, at the prior time point, that is, prior levels of the mediators (e.g., $\mathrm{M} 1_{1}$ ) were controlled when predicting mediators at a later time (e.g., $\mathrm{M} 1_{2}$ ); similarly, effects of $Y_{2}$ were controlled for when predicting $Y_{3}$ (Cole \& Maxwell, 2003). The following diagram illustrates a multiple mediator model and the associated equations. (Note that $\mathbf{X}_{1}$ represents a vector of independent variables, SES/demographic in this case).

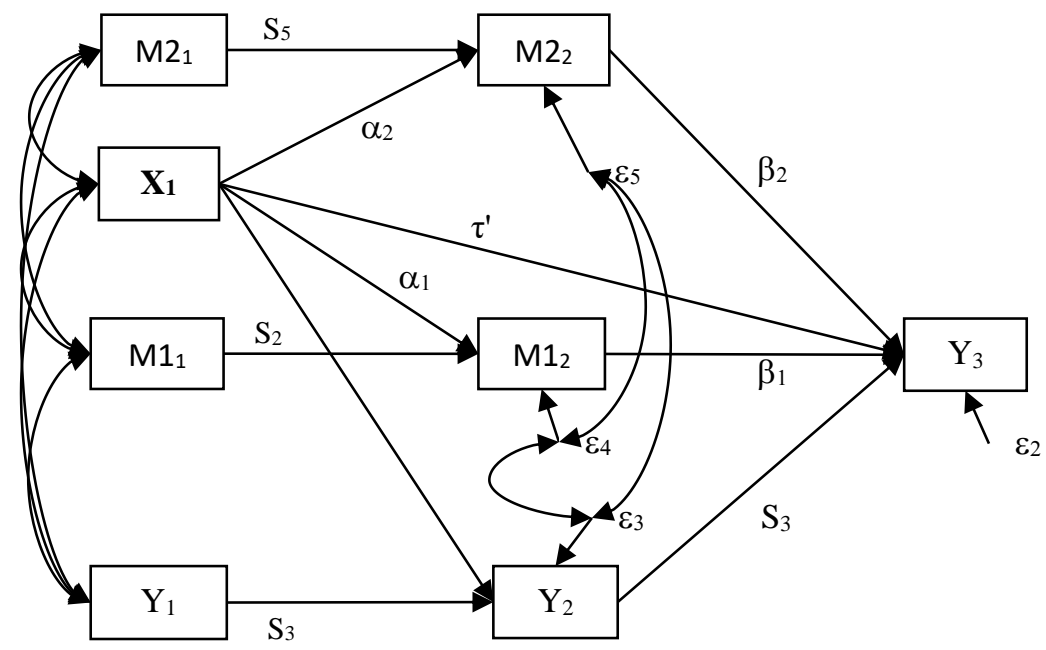

Figure 7: Path diagram for a two mediator model

Note: Two headed curly arrows indicate covariances. All regression paths are not indicated for visual simplicity. In subsequent figures, the $\varepsilon$ 's (errors) are not explicitly indicated.

Regression equations for the two mediator model (Figure 7) are given as:

$$
\begin{array}{lllll}
\mathrm{M} 1_{2}= & \text { Intercept }_{2}+\alpha_{1} \mathrm{X}_{1} & +\mathrm{S}_{2} \mathrm{M} 1_{1}+ & \varepsilon_{4} \\
\mathrm{M} 2_{2}= & \text { Intercept }_{5}+\alpha_{2} \mathrm{X}_{1} & +\mathrm{S}_{5} \mathrm{M} 2_{1}+ & \varepsilon_{5} \\
\mathrm{Y}_{3}= & \text { Intercept }_{3}+\beta_{1} \mathrm{M}_{2}+\beta_{2} \mathrm{M} 2_{2}+\tau^{\prime} \mathrm{X}_{1}+\mathrm{S}_{3} \mathrm{Y}_{2} & \varepsilon_{2}
\end{array}
$$


where equations (1) and (2) are for the effects of $\mathrm{X}$ on $\mathrm{M} 1_{2}$ (mediator one at time two) and $\mathrm{M} 22_{2}$ (mediator two at time two) at Time two respectively and equation (3) is for the effects of $\mathrm{M}_{2}$ and $\mathrm{M} 2_{2}$ on $\mathrm{Y}_{3}$ at Time three controlling for the effects of $\mathrm{X}$ and the effects of the DV at the prior time point $\left(\mathrm{Y}_{2}\right)$ on $\mathrm{Y}_{3}$.

Older approaches to mediation (Baron \& Kenny, 1986; Judd \& Kenny, 1981) require that the total effect $(\tau)$ be significant and the direct effect controlling for the mediator (i.e., $\tau^{\prime}$ ) be non-significant, but this requirements are known to reduce statistical power. Further, because the product of two normal distributions is not necessarily normal, the assumption that the mediated effect divided by its standard error has a normal distribution is incorrect in some situations (MacKinnon, Lockwood, \& Williams, 2004). Therefore, in some situations, mediation can exist even when $\tau^{\prime}$ is significant (i.e., when $\mathrm{X} \rightarrow \mathrm{Y}$ path is significant after controlling for the indirect effect through the mediator) (MacKinnon, 2008). Significance tests of the mediated effects (i.e., $\alpha_{1} \beta_{1}$ or $\alpha_{2} \beta_{2}$ ) were tested by computing z-scores and obtaining their $\mathrm{p}$-values. Mplus, the analysis program used to test indirect effects, uses the multivariate delta method (Sobel, 1982, 1987)

\section{Longitudinal Mediation with Binary Outcome Variables}

Overnight hospitalization was a binary response variable in this study. A binary outcome variable has logistic distribution rather than normal distribution as assumed when modeling with a continuous outcome variable. Logistic regression is the appropriate method for modeling when the dependent variable is binary. The general 
logistic regression model for the binary variable without the mediator variable is given by,

$$
\operatorname{Logit}\left(\operatorname{Pr}\left\{Y_{i}=1 \mid X_{i}\right\}\right)
$$

where $Y_{i}$ represents outcome scores for the hospitalization variable at different time points and $\mathbf{X}$ is a vector of predictors including socioeconomic status variables as well as demographic variables (age and gender).

In the present study, the binary outcome variable, $Y$, (Note $\mathrm{Y}$ is used in place of overnight hospitalization and $\mathrm{X}$ for SES/demographic predictors for convenience) was treated as estimating an underlying latent continuous variable, $Y^{*}$, and the above equation in its latent form following MacKinnon, Lockwood, Brown, Wang, and Hoffman (2007) is given as,

$$
\mathrm{Y}_{\mathrm{i}}^{*}=\alpha_{1}+\tau(\mathrm{X})+\varepsilon_{1}
$$

where $\alpha$ represents the intercept, $\tau$ represents the regression coefficient and $\varepsilon_{1}$ represents the residual variability of the observed outcome with standard logistic distribution. The appropriate link functions for the transformed equation are the logit link function or the probit link function. In this study, the probit link function was used for reasons described below. The equations for the mediator model were similar to the mediation equations provided in the above section.

Predicting categorical variables and interpreting results presents challenges because of violation of distributional assumptions. Thus, when the outcome variable has fewer than five categories, using Muthen's categorical variable methodology (CVM) (Muthen, 1984) implemented in Mplus with Weighted Least Squares Mean-Variance 
adjusted (WLSMV) estimator and theta parameterization is recommended (Finney \& DiStefano, 2006). This method was used to compute mean and variance adjusted chisquare statistic, and other goodness of fit indices including the Cumulative Fit Index (CFI) and the Root Mean Square Error of Approximation (RMSEA) as alternative measures of fit as suggested (Hu \& Bentler, 1999). WLSMV can be used with moderately non-normal and severely non-normal data with fewer than five categories (Finney \& DiStefano, 2006) and it was convenient for modeling the overnight hospitalization variable in this study. The regression coefficients Mplus provides with the use of categorical binary dependent variables and estimator WLSMV are probit coefficients. Note that results of probit or logit link functions in such modeling lead to similar statistical conclusions.

\section{Mediation in Latent Growth Curve Modeling Framework}

Growth curve modeling approach provides the advantage of investigating individual differences in the trajectory of change over time (Cheong et al., 2003). Latent growth curve modeling (LGM) is designed to examine change over three or more time points, and can be adapted for linear or nonlinear trajectories of change. The LGM approach, implemented in traditional SEM framework, is also a useful technique when the objective is to determine inter-individual variability in the slope and the mean (Selig \& Preacher, 2009) of the factors. The LGM method has advantages over ANOVA and autoregressive models where individual differences in growth and change in the measures over time are not explicitly modeled. Thus, LGM can be used to test longitudinal mediation models where the independent variables, the mediators and the outcome 
variables are repeatedly measured (MacKinnon, 2008; Muthen \& Curran, 1997; Singer \& Willett, 2003) but can also be used when the independent variable is measured at one time point but the mediator and outcome variables are measured at several time points. Basically, LGM is used to investigate if the relation between an independent variable and a dependent variable is totally or partially accounted for by the mediating variable (MacKinnon, 2008).

In the LGM framework, mediation analysis examines whether the independent variable affects the growth trajectory of the mediating variable and whether the growth trajectory of the mediating variable, in turn, affects the growth trajectory of the dependent variable. The growth factors are represented by latent factors - the intercept factor representing the initial status of the growth trajectory at Time 1, and the slope factor that defines the shape of the trajectory of change over time. The growth curve model includes relations between various factors: the slope factor of the mediating variable as well as the slope factor of the dependent variable (MacKinnon, 2008) and covariances between the intercept factors as well as the slope factors can be modeled. Further, LGM provides group-level statistics that are useful in assessing differential trajectories in the dependent variable by groups. By investigating how SES is related to trajectories of change in the mediating variables, which in turn are related to the trajectory of change in the outcome variable, this study examined the role of the mediating variables in the differential growth in physical limitations by race/ethnicity. The general model for individual growth process of the mediating variable with five time points ( 5 waves) was given as,

$$
M_{i}=V_{i}^{(m)}+\Lambda^{(m)} \eta_{i}^{(m)}+\varepsilon_{i}^{(m)} \text {, }
$$


where $\mathrm{M}_{\mathrm{i}}$ was a 5 x 1 repeated measure of the mediator variables, $\mathrm{v}_{\mathrm{i}}{ }^{(\mathrm{m})}$ was the vector of the common intercepts of the mediator variables, $\Lambda^{(\mathrm{m})}$ was a $5 \times 2$ matrix for the factor loadings on the two growth factors, $\eta_{i}^{(m)}$ is a $2 \times 1$ vector of the latent growth factors of the mediators, and $\varepsilon_{\mathrm{i}}^{(\mathrm{m})}$ is a $5 \times 1$ vector of measurement errors of the mediators. The loadings for the intercept factor were set equal to 1 whereas the loadings for the slope factor were set to values of the time variable (i.e., $0,1,2,3,4)$ representing the five data collection time points. The mean of the factor provides information about average linear change when the codes $0,1,2,3,4$ are used for the slope factor. The variance of the factor indicates whether there is significant variability among individuals in growth with respect to outcomes (Duncan, Duncan, Strycker, Li, \& Alpert, 1999). Similarly, the growth process for the outcome variable (i.e., physical impairment status) was modeled as above. Figure 8 provides an illustrative example. 


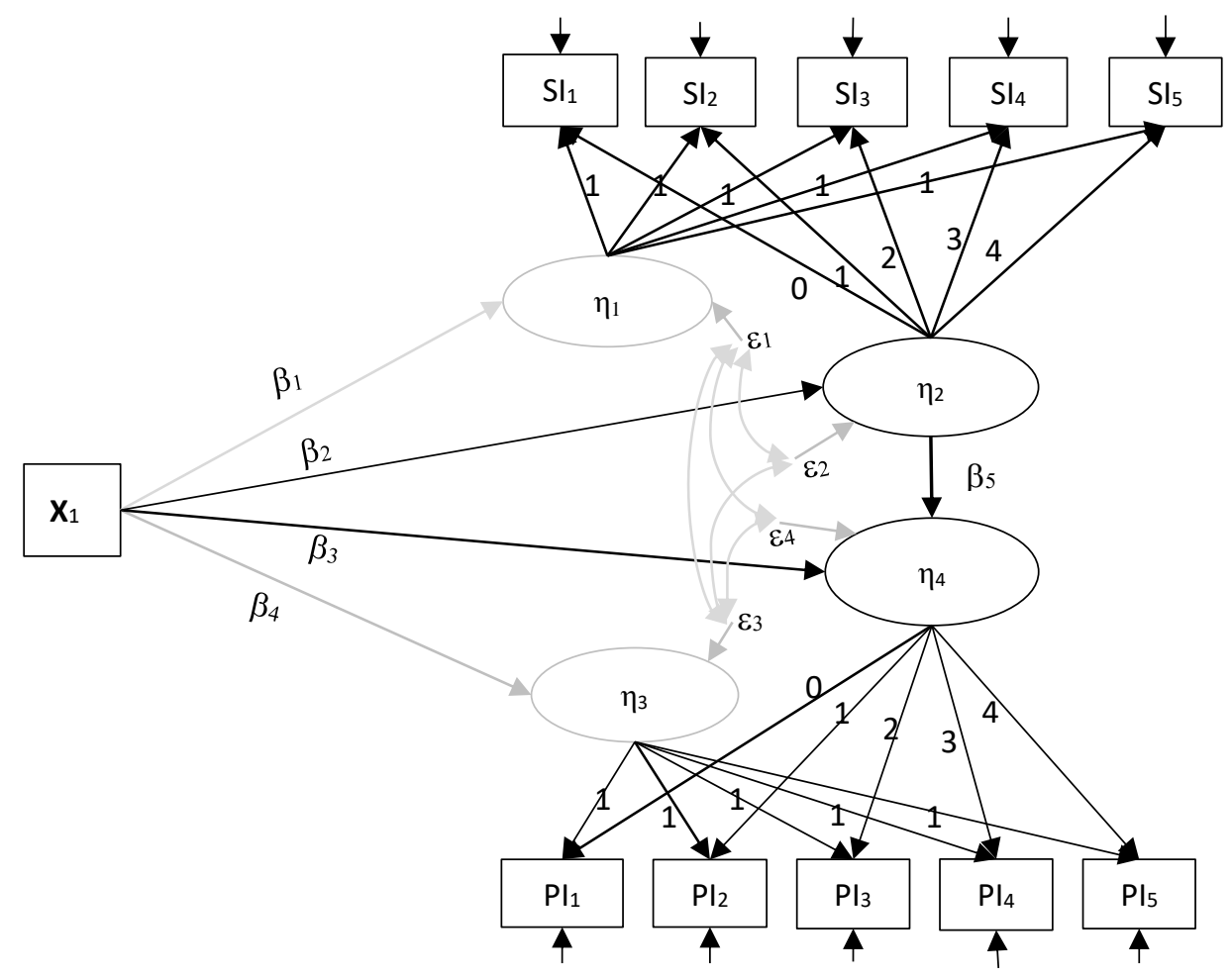

Figure 8: First order growth curve model

Note: SI=social integration, $\mathrm{PI}=$ physical impairment, $\eta_{1} \& \eta_{3}$ intercepts and $\eta_{2} \& \eta_{4}$ slopes. All paths, variances, covariances and residual errors are not labeled; $\mathbf{X}_{1}=$ predictor variables.

In Figure 8, the equations for the slope factors for SI (social integration) and PI

(physical impairment) are given, respectively, as

$$
\begin{gathered}
\eta_{2}=\alpha_{2}+\beta_{2} X_{1}+\varepsilon_{2} \\
\eta_{4}=\alpha_{4}+\beta_{3} X_{1}+\beta_{5} \eta_{2}+\varepsilon_{4}
\end{gathered}
$$

The product of the coefficients $\beta_{2}$ and $\beta_{5}$ (i.e., $\beta_{2} * \beta_{5}$ ) indicate the indirect effect and the coefficient $\beta_{3}$ indicates the direct effect.

\section{Procedures for Testing Mediation in LGM Framework}

Testing mediation analyses in LGM involves several steps. In the first step, the change trajectory of each mediator and outcome process is investigated. Examination of growth trajectory of the mediators and physical impairment status (outcome variable) 
were conducted for each race/ethnic group. These unconditional models provide evidence for initial state and growth patterns of the social integration factors and psychological factors, and initial state and change patterns of physical impairment status. The aim of this step was to test whether the hypothesized trajectory of linear change, for example, in the physical impairment status case, fit the data well.

Fit indices including $\chi^{2}$ (chi-square) statistic, root mean square error of approximation (RMSEA) and cumulative fit index (CFI) were computed to examine model fit. Estimates of the mediated effect and standard errors were used to obtain zscores and test significance of path coefficients using p-value. As described above, Mplus uses the delta method in significance test of indirect effects. In models indicating significant indirect effects, follow up analysis using chi-square test of nested models examining multiple group path invariance were conducted.

\subsubsection{Reconstructability Analysis (RA)}

Reconstructability Analysis (RA) was originated by (Ashby, 1964) and later advanced in a number of ways at different places (Klir, 1986; Krippendorff, 1986) including at the Systems Science PhD Program at Portland State University (Zwick, 2001). RA is an information and graph theory based method which has been successfully used in multivariate, multidimensional data modeling (Kramer, Westaway, Zwick, \& Shervais, 2012; Shervais \& Zwick, 2003; Zwick, 2004, 2011; Zwick \& Shu, 1996). Elaborate description of the overall method is provided elsewhere (e.g., Zwick, 2004). In the information theoretic approach, RA method decomposes a probability or frequency 
distribution into component distributions from which a model is derived by composition of the component distributions by way of maximum entropy. Therefore, RA in this sense does statistical multivariate analysis and resembles log-linear methods (Kramer, Westaway, Zwick, \& Shervais, 2012).

In this study, RA was used for exploratory modeling of both neutral and directed systems. In directed system analysis a pre-specified model was tested. Associations between variables are detected but the magnitude is not provided in neutral search. However, magnitude can be obtained by using a directed system search. When using directed system modeling in RA, one can compute uncertainty reduction in the dependent variable due to the predictor variables. RA requires that variables be discrete (binary or multi-category), a process that may lead to loss of information, but this loss can be compensated for by the possibility of detecting non-linear multi-variable interaction effects among the predictor variables that are not hypothesized in advance (Zwick, 2004).

One of the uses of RA is dimension reduction. In searching the lattice of structures where several variables are involved, RA screens for variables (Zwick, 2004) and suggests the most important predictors and further modeling involving these variables can be done based on the selected variables. Ideally, all models found in exploratory searches should be tested in new data to assess generalizability.

Analyses were conducted using OCCAM (ㅁganizational Complexity

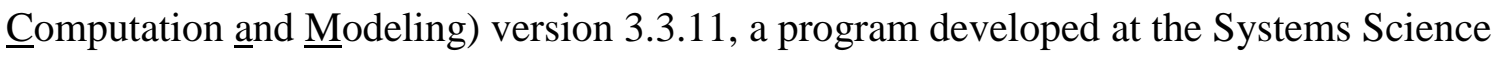
Graduate Program (originally by Zwick and Hosseini), Portland State University. 


\section{Analytic procedures in $R A$}

Model selection in RA is dependent on N (sample size). Ideally, equal sample size for any two racial/ethnic groups compared is thus preferred. The original sample sizes for Whites, Blacks and Hispanics were different, which then obtained different RA models making racial/ethnic comparison of predictive variables untenable. Data in this study were, therefore, matched to achieve comparable groups on covariate distribution and sample sizes.

The matching process sought to obtain equal or nearly equal sample sizes with similar distributional balance on covariates for comparison groups so that one group that is compared to another group has similar covariate distributions for model search in RA. One of the approaches for matching is using propensity scores. Propensity score is simply the probability of a case being in the treatment or control group given a vector of covariates. Further details on propensity score matching for causal analysis are provided in Rubin (Rubin, 1997; Rubin, 1976; Diamond \& Sekhon, 2013), and R-packages that implement various matching schemes can be found in (Ho, Imai, King, \& Stuart, 2007), and (Sekhon, 2011). Propensity scores can be computed using a logistic regression with treatment variable as the dependent variable and the covariates to be balanced for the treatment and the control group as independent variables. In this study, the binary variable indicating race/ethnicity (i.e., White vs Hispanic; White vs Black; and Black vs Hispanic) was used as the dependent variable (i.e., as a 'treatment' variable), whereas, income, wealth, education, job status, age and gender were used as independent variables to obtain data where Whites and Hispanics were similar in these covariates (and similarly 
for other pairs of groups). Steps for preprocessing and matching of data are provided below.

The process for matching required that there be no missing data, thus, using Rpackage Optmatch version 0.9-6 (Hansen \& Klopfer, 2006), missing values for income, wealth, employment, and education were filled with non-informative values (mean of the available/non-missing column values) based on column values with the following code in R statistical software:

Data1 $=$ fill.NAs $($ Data, data $=$ NULL, all. covs $=$ FALSE, contrasts $\cdot \arg =$ NULL $)$

- "Data" is the original dataset for the White (race=0) and Hispanic (race=1) groups.

- "Data1" is a new data set where all missing values for income, wealth, education, job status, age and gender are filled in with values computed using the column values for each variable because computing propensity score and matching requires that there be no missing data.

The next step was to compute propensity scores and match the cases based on these scores. Using the R-package MatchIt (Ho et al., 2007), the code below first computes propensity scores (i.e., the probability of race $=0$ or race $=1$ given the covariates), and then, using these computed values, matches Whites and Hispanics, retaining only Whites and Hispanics similar on the covariates (see Figures 9 and 10 for an example histogram after matching). The code below computes propensity scores and matches cases using nearest neighbor method for two groups (e.g. Whites and Hispanics)

Data2 $<-$ MatchIt $\left(\right.$ race $\sim$ income $+\begin{array}{c}\text { wealth }+ \text { education }+ \text { job status }+ \text { age }+ \text { gender, data=Data1, } \\ \text { method="nearest" })\end{array}$ 
- "Data1" is the new data obtained using the first code above.

- "Data2" is the new matched data set that was used in RA. Note that the imputed values were dropped after obtaining a matched data.

Using the above steps, three data sets were obtained where Whites and Hispanics had similar N (N=641); Whites and Blacks had similar N (N=1084); and Blacks and Hispanics had similar $\mathrm{N}(\mathrm{N}=641)$.

For example, distributions of income and wealth for the White/Hispanic dataset, after matching, are given below showing similarity of both groups falling in each bin. Note that the sole objective of matching in this study was to obtain groups with similar $\mathrm{N}$ such that the covariate distribution was similar as indicated in the histograms below.

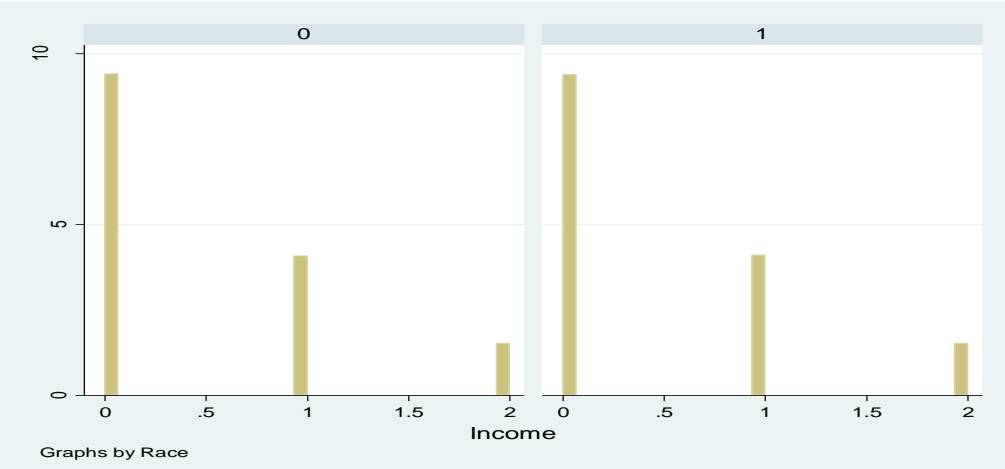

Figure 9: Histogram, Whites (left panel) and Hispanics in each income bin after matching.

Note: White $\mathrm{N}=614$, Hispanic $\mathrm{N}=611$. Bins 0 is for $<\$ 26,000$; 1 is for $\geq$ to $\$ 26,000$ but $\leq \$ 54,000$; and 2 is for $\geq \$ 54,000$ 


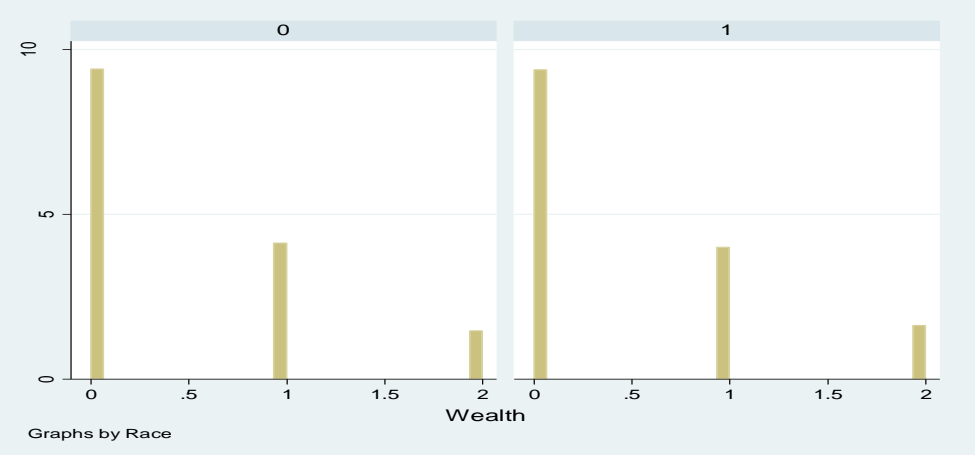

Figure 10: Histogram, Whites (left panel) and Hispanics in each wealth bin after matching Note: White $\mathrm{N}=614$, Hispanic $\mathrm{N}=611$. Bin 0 is for less than $\$ 100,000 ; 1$ is for $\geq \$ 100,000$ but $\leq 350,000$; and 2 is for $\geq \$ 350,000$

This process essentially provided Whites that were similar to Blacks and Hispanics on the covariates and Blacks that were similar to Hispanics in the covariates because the sample size for Hispanics was smaller than the White and Black samples. That is, Hispanics that matched the White and Black group were similar. The next sections describe the purpose of using RA, types of models and model selection criteria.

RA was used to examine two classes of models, namely, variable based models without loops and VB models with loops, which allow for a coarse grained modeling and refined modeling processes respectively (Kramer et al., 2012). Models without loops do variable selection and models with loops are the more detailed models. Because all variables in models without loops also appeared in models with loops, and because models with loops had a better BIC, variable based models without loops were not reported in this study. 


\section{$\boldsymbol{R A}$ model types}

In RA, Neutral system is an approach where all variables remain neutral (i.e., no IV/DV distinction is made), whereas in a directed system, a dependent variable and one or several independent variables are specified. Both modeling approaches were used in the present study.

Neutral system models. In neutral system modeling, RA was used to identify SES/demographic variables strongly associated with the mediator (for instance psychological variable) so that the associated SES/demographic variables are used as IVs and the associated $\mathrm{P}_{i}$ (where $i$ indicates selected variable from $\mathrm{P}$ ) variable is used as a DV in the path analysis sections. This step was repeated for the mediator variables B and S to identify $\mathrm{B}_{i}$ and $\mathrm{S}_{i}$ variables (Figure 11).

Directed system models. In RA, directed system search requires specification of a dependent variable (DV) and one or several independent variables (IVs). The objective of this step was for prediction of outcome variables (i.e., physical impairment and overnight hospitalization) given demographic, socioeconomic, social, psychological and behavioral variables. This approach provides predictive variables (single predictors or interaction effects) and computes uncertainty reduction in the DV due to the selected predictors. Directed system modeling was also used in confirmatory mode in path analysis (Figure 11) to simply compute uncertainty reduction in the mediator variable and outcome variable as explained below in the path value calculations section. 


\section{Controlling for the third variable in RA}

In path analysis, the effect of the mediator on the outcome variable was adjusted for the direct effect of the selected SES variable on the outcome variable. Similarly, the effect of the SES variable on the outcome variable was adjusted for the effect of the mediator on the outcome variable. The general formula and the procedures for computing adjusted uncertainty reductions are provided below. Detailed procedures of this method are given elsewhere (Kramer et al., 2012).

This study assumed a recursive model as indicated in Figure 11. To present the procedure of controlling for a variable in RA, three hypothetical variables A for SES, B for mediator and $\mathrm{Z}$ for outcome variables are considered and represented as below.

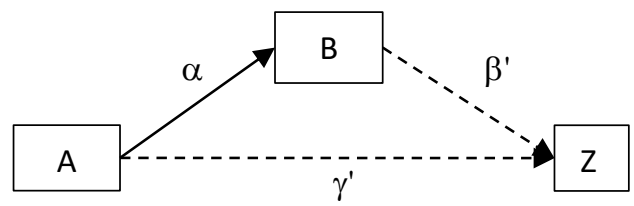

Figure 11: Recursive path model illustrating RA uncertainty reductions

\section{Path value calculations in $R A$}

$\underline{\text { A to } B \text { path }}$

$\alpha=\mathrm{T}(\mathrm{A}: \mathrm{B})$, Transmission between $\mathrm{A}$ and $\mathrm{B}$. Note $\mathrm{B}$ is the $\mathrm{DV}$.

$$
=U(A)+U(B)-U(A B)
$$

Where $U$ (uncertainty or entropy of A [also for uncertainty of B]) is given by:

$$
U(A)=-\sum_{i=0}^{n} \mathrm{p}(\mathrm{A} i) \log p(A i)
$$

\section{$\underline{B}$ to $\boldsymbol{Z}$ path controlling for the effects of $A$ on $Z$.}

Note: A \& B are IVs and Z is DV in the computations below. Path $\beta$ represents the effect of $\mathrm{B}$ on $\mathrm{Z}$ controlling for the effect of $\mathrm{A}$ on $\mathrm{Z}$. Thus, 


$$
\beta^{\prime}=T_{A}(B: Z),
$$

$\mathrm{T}$ is transmission or mutual information between $\mathrm{B}$ and $\mathrm{Z}$ controlling for $\mathrm{A}$.

$$
\begin{gathered}
\mathrm{T}_{\mathrm{A}}(\mathrm{B}: \mathrm{Z})=\mathrm{T}(\mathrm{AB}: \mathrm{AZ})=\mathrm{T}(\mathrm{AB}: \mathrm{Z})(1-\mathrm{I}(\mathrm{AB}: \mathrm{AZ})) \\
\beta^{\prime}=\mathrm{T}_{\mathrm{A}}(\mathrm{B}: \mathrm{Z}) / \mathrm{U}(\mathrm{Z})
\end{gathered}
$$

$=$ fractional reduction of the uncertainty of $\mathrm{Z}$, given $\mathrm{B}$, controlling for $\mathrm{A}$.

\section{$\underline{A}$ to $\boldsymbol{Z}$ path controlling for the effect of $B$ on $Z$}

$\gamma^{\prime}=T_{B}(A: Z) / U(Z)$, fractional reduction of the uncertainty of $Z$, given A, controlling for $B$,

$$
\gamma^{\prime}=\mathrm{T}_{\mathrm{B}}(\mathrm{A}: \mathrm{Z}) / \mathrm{U}(\mathrm{Z})=\mathrm{T}(\mathrm{AB}: \mathrm{Z})[1-\mathrm{I}(\mathrm{AB}: \mathrm{BZ})] / \mathrm{H}(\mathrm{Z})
$$

Where,

$\mathrm{T}(\mathrm{AB}: \mathrm{Z})$ is transmission (or mutual information) between $\mathrm{AB}$ and $\mathrm{Z}$

$\mathrm{I}(\mathrm{AB}: \mathrm{BZ})$ and $\mathrm{I}(\mathrm{AB}: \mathrm{AZ})$ indicate information gain about $\mathrm{Z}$ due to $\mathrm{B}$ and $\mathrm{A}$ respectively.

$\mathrm{U}(\mathrm{Z})$ is uncertainty (entropy) of the DV

(In the RA software, fractional entropy (uncertainty) reductions are expressed as \% reductions.)

\section{Model selection criterion in $R A$}

Several model selection criteria are available in the OCCAM implementation of RA. The frequently used criteria are the Akaike Information Criterion (AIC), the Bayesian Information Criterion (BIC) and incremental p-value. All three measures assess goodness of fit of models by integrating error and complexity in various ways. All three criteria penalize models for complexity at varying degrees but BIC is the most conservative criterion (it penalizes models for complexity the most) and thus the most reliable of the criteria. Actual model selection is based on the difference or change in BIC and AIC between two models (reference model and selected model). AIC and BIC do not 
require that models being compared be hierarchically nested, however, incremental pvalue requires that models being compared be hierarchically nested. When best models are suggested based on $\triangle \mathrm{AIC}$ and $\triangle \mathrm{BIC}$, these best models have maximum values (Zwick, 2001, 2004). In this study, $\triangle \mathrm{BIC}$ was used to select models. In directed models, variables that reduce the highest uncertainty in the dependent variable, if these reductions are statistically significant at the 0.05 level, were selected.

Uncertainty can be conceptualized as absence of information about a dependent variable. Adding explanatory variables to the model reduce this uncertainty if the variables added are predictive of the dependent variable. The RA output provides percent uncertainty reduction as variables are added to the independence model. Uncertainty, in this sense, applies to nominal variables and does not depend on any metric. The magnitude of uncertainty reductions are different from the magnitude required for considerable reduction of variance in regression methods, because RA calculations involve a $\log$ term and a small percent uncertainty reduction could potentially be important. One rule of thumb is to consider percent uncertainty reduction of 8 and above to be sizable (Kramer et al., 2012). The difference in degrees of freedom between the selected model and the reference model is given as change in degrees of freedom $(\Delta \mathrm{df})$ and indicates model complexity - models with higher $\Delta \mathrm{df}$ are more complex and viceversa. Models with lower delta degrees of freedom are preferred.

When searching for best models from a large family of models, one or more models could be selected as best simply due to chance. In regression methods, a 
Bonferroni correction is applied to adjust for type I error rates in family-wise comparison of models. The standard methods that use Bonferroni adjustment are set in confirmatory mode and the correction is essential. However, RA is an exploratory modeling technique where the best models selected are, ideally, expected to be validated using test-data (i.e., part of the data that has been set aside and not used in training). The p-value that OCCAM prints is not Bonferroni-corrected. Thus, the most conservative model selection criterion, namely BIC, was used to minimize chances of selecting an overfitting model. $\mathrm{BIC}$, in fact, usually underfits, and this guards to a certain degree against selecting a model that has a low p-value merely by chance due to the evaluation of many models. Nevertheless, in RA, any model selected using training data needs to be tested to validate model fit. Such confirmatory tests are not part of this study, thus all models proposed by RA should be viewed as only exploratory results providing hypotheses that may be tested in the future. 


\section{CHAPTER 5.0: RESULTS FOR STRUCTURAL EQUATION MODELING}

\subsection{Analysis Overview}

Lagged path modeling and latent growth curve modeling approaches were used to examine indirect relationship of income, wealth, employment status, and education level with physical impairment via social integration (SI), psychological factors (depressive symptoms [DS]), and behavioral factors including body mass index (BMI) and drinking (Acl). Lagged path modeling approach allows for evaluation of direct and indirect effects controlling for the dependent variable at the previous time point. Using these four SES and two demographic variables (age and gender) simultaneously had the advantage of examining effects of each variable adjusted for the effects of all other variables in the models. In examining lagged path models, I first conducted lagged path analysis for the total sample and then extended the model to multiple groups to simultaneously examine indirect paths from each SES variable at the first time point to physical impairment variable at the third time point via the proposed mediators at the second time point and compare these paths by race/ethnicity. Unstandardized ( $\beta$ ) path coefficients with their standard errors and standardized $\left(\beta^{*}\right)$ path coefficients are provided in the accompanying figures for significant indirect paths. For clarity, subscripts $w, b$ and $h$ are used with some parameters to indicate results for white, Black and Hispanic groups respectively. Additional results are provided in Appendix B.

The second outcome variable in this study was overnight hospitalization and similar lagged path modeling approach was used to examine indirect association of each SES variable with overnight hospitalization at the third time point via each of the 
mediator variables at the second time point. But, because the overnight hospitalization variable was binary, adjustments to modeling were made (as described in Chapter 4) due to violation of assumptions for ordinary least squares approach.

This study used the multivariate delta method (Sobel, 1982, 1987) to assess significant indirect relationships, that is, to test significance of product of the coefficients. The SEM program, Mplus, computes robust standard errors when the MLR estimator is specified for slightly non-normal data with missing values. This method provides similar results to the bootstrap technique which is considered among the best approaches in estimating robust standard errors for statistical analysis with missing data.

Lagged path models focus on the stability of rank order of respondents on variables across time rather than the direction of change over time (MacKinnon, 2008). To supplement the lagged path model analyses by taking advantage of longitudinal data to examine growth patterns and the direction of the trajectory of social integration and depressive symptoms in each race/ethnic group, I conducted a latent growth curve mediation analysis. These two latent growth curve models examined relationships of the growth of social integration and depressive symptoms to the growth of physical impairment conditional on SES and demographic factors. Indirect effects of SES on the growth factor of physical impairment via the growth factor in social integration and depressive symptoms were conducted.

In all models, chi-square was significant which should not be surprising given the complexity of the models and the large sample sizes. Evaluation of model fit, therefore, 
was based on alternative fit indices as suggested by $\mathrm{Hu}$ and Bentler including comparative fit index (CFI) and the standardized root mean square residual (SRMR) for the continuous outcome variable (i.e., physical impairment) (Hu \& Bentler, 1999). In general, SRMR values less than 0.08 and CFI values greater than 0.95 indicate good fit. Follow-up analyses using chi-square test of nested models for paths suggesting significant indirect association were conducted to further examine equality of paths across race/ethnicity.

Physical impairment status was assumed to take longer time to manifest itself as a limitation in activities of daily living. So, for physical impairment outcome, time between successive data collection points for the lagged path models was four years, while it was two years for the growth curve models. Lag time between adjacent data collection points for the overnight hospitalization outcome variable was two years.

Baseline characteristics of respondents exclude cases that used psychotropic medications, or had emotional problems and saw a psychiatrist or psychologist. These results did not alter statistical conclusions where the respondents were excluded. Analyses in this section of the study take into account the complex sampling design by incorporating baseline probability weights, stratification and clustering variables that the Health and Retirement Study (HRS) provided with the public release data sets. Analyses were conducted using Mplus version 7.4 (Muthén \& Muthén, 1998-2015), SPSS 21 (IBM Corp., 2012) and Stata (StataCorp., 2013). 


\subsection{Baseline Sample Characteristics}

Table 2 provides baseline characteristics of respondents for the total sample and by race/ethnicity for all variables used in the specification of the models. Consistent with previous studies, mean income, education and wealth were higher for Whites, whereas Blacks and Hispanics had comparable income and wealth levels. Blacks had higher education levels compared to Hispanics, however. The proportion employed was higher among Whites (37.5\%) followed by Blacks (34.3\%) and Hispanics (30.0\%). Approximately, only a third of the respondents were employed in each race/ethnic category indicating that larger proportion of the respondents may be retirees.

The first type of mediator was social integration, an index constructed using five individual questions and ranged from 0 (low integration) to 5 (high integration). Mean baseline social integration score for Whites $(\mathrm{M}=2.68, \mathrm{SE}=0.018)$ was slightly larger than the one for Blacks $(\mathrm{M}=2.38, \mathrm{SE}=0.047)$ and Hispanics $(\mathrm{M}=2.35, \mathrm{SE}=0.057)$ but Hispanics and Blacks had essentially similar mean levels of social integration.

The second type of mediator was depressive symptoms, a latent variable with three factor indicators constructed using the short version of the Center for Epidemiological Studies Depression (CES-D) scale comprising of eight binary questions. Baseline score for the latent depressive symptoms variable was highest for Hispanics $(\mathrm{M}=0.24, \mathrm{SE}=0.014)$, closely followed by the mean score for Blacks $(\mathrm{M}=0.21$, $\mathrm{SE}=0.010)$. Mean depressive symptoms score $(\mathrm{M}=0.15, \mathrm{SE}=0.005)$ in the White group was lower than the mean depressive symptoms scores for Hispanics and Blacks, however. 
Table 2: Baseline sample characteristics

\begin{tabular}{|c|c|c|c|c|}
\hline \multirow{2}{*}{ Variable } & Total & White & Black & Hispanic \\
\hline & Mean (SE or \%) & Mean (SE or \%) & Mean (SE or \%) & Mean (SE or \%) \\
\hline \multicolumn{5}{|l|}{ SES/demographic } \\
\hline Age & $65.59(0.039)$ & $65.68(0.051)$ & $65.50(0.117)$ & $65.21(0.145)$ \\
\hline Female, n (\%) & $4154(54.13)$ & $3006(52.58)$ & $748(60.42)$ & $400(55.63)$ \\
\hline Income & $10.44(0.031)$ & $10.57(0.031)$ & $9.83(0.057)$ & $9.62(0.117)$ \\
\hline Wealth & $10.84(0.093)$ & $11.36(0.095)$ & $8.24(0.230)$ & $8.40(0.241)$ \\
\hline Education & $12.45(0.092)$ & $12.88(0.077)$ & $11.48(0.112)$ & $8.57(0.361)$ \\
\hline Employed, n (\%) & $2472(36.27)$ & $1901(37.45)$ & $375(34.34)$ & $188(30.03)$ \\
\hline \multicolumn{5}{|c|}{ Mediating variables } \\
\hline $\mathrm{SI}_{\mathrm{t}=1}$ & $2.63(0.017)$ & $2.68(0.018)$ & $2.38(0.047)$ & $2.35(0.057)$ \\
\hline $\mathrm{DS}_{\mathrm{t}=1}$ & $0.16(0.004)$ & $0.15(0.004)$ & $0.21(0.009)$ & $0.25(0.016)$ \\
\hline $\mathrm{BMI}_{\mathrm{t}=1}$ & $27.78(0.083)$ & $27.54(0.088)$ & $29.31(0.181)$ & $28.43(0.295)$ \\
\hline $\operatorname{Alc}_{t=1}$ & $0.37(0.013)$ & $0.40(0.015)$ & $0.22(0.024)$ & $0.24(0.032)$ \\
\hline \multicolumn{5}{|c|}{ Outcome variables } \\
\hline $\mathrm{PI}_{\mathrm{t}=1}$ & $0.24(0.011)$ & $0.21(0.013)$ & $0.41(0.030)$ & $0.43(0.032)$ \\
\hline $\mathrm{OH}_{\mathrm{t}=1}, \mathrm{n}(\%)$ & $1294(20.10)$ & $975(20.24)$ & $208(20.04)$ & $111(19.01)$ \\
\hline
\end{tabular}

Note: $\mathrm{t}=1$ data collection time indicator; $\mathbf{S I}=$ social integration, $\mathbf{D S}=$ depressive symptoms, $\mathbf{B M I}=$ body mass index, Alc=alcoholic beverage use, $\mathbf{P I}=$ physical impairment $\& \mathbf{O H}=$ overnight hospitalization.

The third type of mediator was comprised of behavioral factors which included body mass index (BMI) and level of alcohol use (drinking). Compared to the mean BMI score in the White group $(\mathrm{M}=27.54, \mathrm{SE}=0.088)$, mean $\mathrm{BMI}$ scores for Hispanics $(\mathrm{M}=28.43, \mathrm{SE}=0.295)$ and Blacks $(\mathrm{M}=29.13, \mathrm{SE}=0.181)$ were slightly higher. Mean levels of alcoholic beverage use in the White group was nearly two times higher 
$(\mathrm{M}=0.40, \mathrm{SE}=0.015)$ than that in Blacks $(\mathrm{M}=0.22, \mathrm{SE}=0.024)$ and Hispanics $(\mathrm{M}=0.24$, $\mathrm{SE}=0.032)$.

The outcome variables in these models were levels of physical impairment using an index derived from scores on five Activities of Daily Living (ADL) measures which ranged from 0 to 6 , and overnight hospitalization which was a binary $(1 / 0=y e s /$ no $)$ variable. Higher scores of physical impairment indicate higher levels of physical impairment. Mean baseline scores for levels of physical impairment among Blacks $(\mathrm{M}=0.41, \mathrm{SE}=0.030)$ and Hispanics $(\mathrm{M}=0.43, \mathrm{SE}=0.032)$ were comparable but were nearly two times higher than the mean physical impairment scores in the White group $(\mathrm{M}=0.21, \mathrm{SE}=0.013)$. Proportion of Whites, Blacks and Hispanics with at least one overnight hospitalization were $0.20,0.20$ and 0.19 (or 20\%, 20\%, 19\%) respectively, indicating that essentially all groups had similar rates of overnight hospitalization at baseline.

\subsection{Mean Scores (or Proportions) of Mediating and Outcome Variables over Time}

Table 3 provides mean scores by race/ethnicity and for the total sample for the mediators including social integration, depressive symptoms, alcohol use, body mass index, and the outcome variable, physical impairment, at subsequent time points. As in baseline, the mean score for the social integration variable at the second time point was higher for Whites $(M=2.61, S E=0.025)$, followed by the mean score for Blacks $(M=2.28$, $\mathrm{SE}=0.046)$ and Hispanics $(\mathrm{M}=2.15, \mathrm{SE}=0.043)$. Mean scores for social $(\mathrm{SI})$, psychological (DS) and physical impairment (PI) factors are provided in Figure 12. 


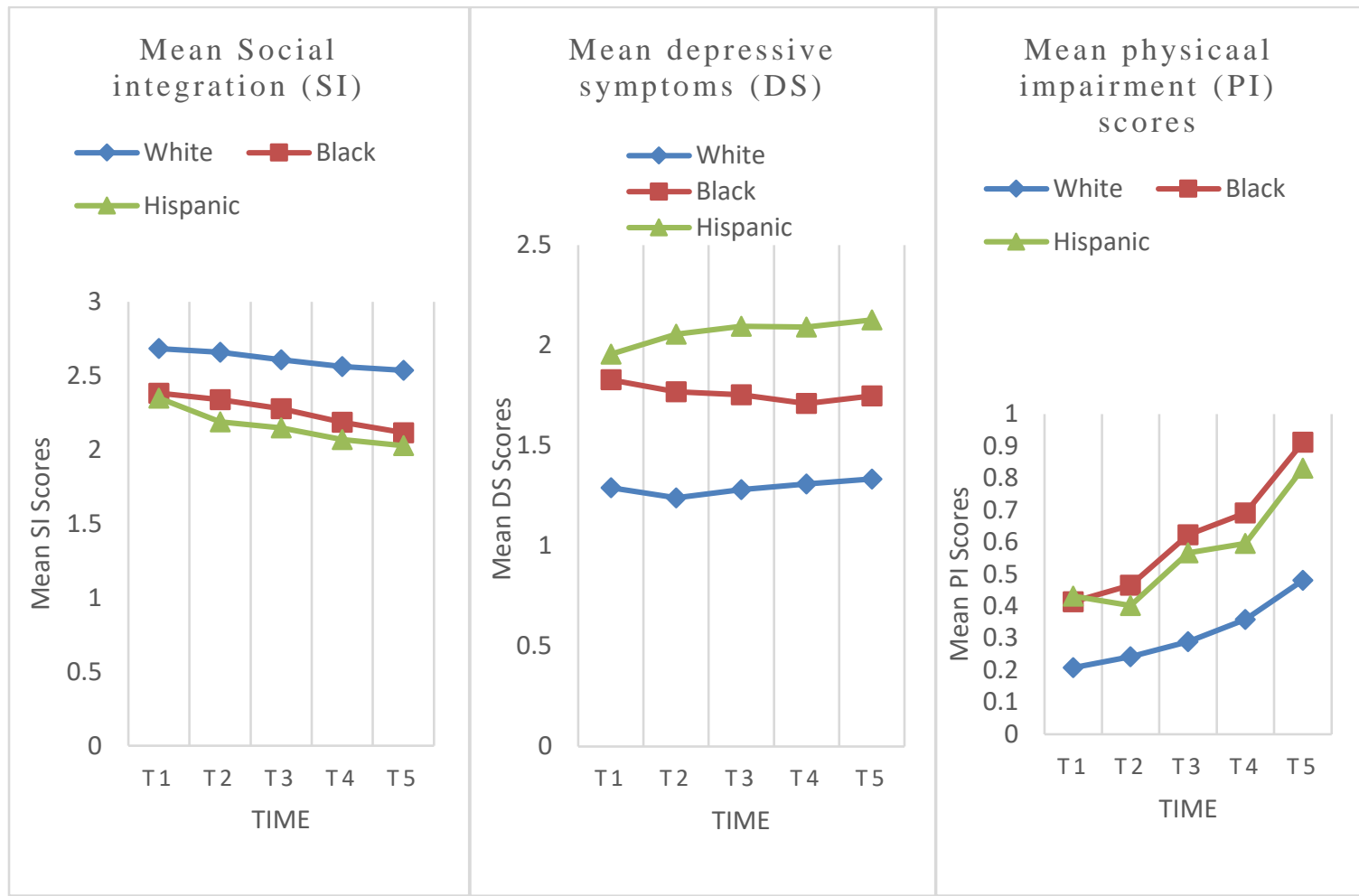

Figure 12: Estimated mean change in social integration (SI), physical impairment (PI) and depressive symptoms (DS) level over time

Figure 12 indicates that the social integration index seemed to decline over time for all groups (top left panel). Whites followed by Blacks and Hispanics had higher social integration index and the scores appeared to decline over time. Physical impairment, however, appeared to increase steadily over time for all groups. Mean depressive symptoms overtime were higher for Hispanics, followed by Blacks and mean score for Whites was substantially lower. However, mean growth in depressive symptoms appeared to be steady which may result in poor fit in the growth curve models or fail to indicate variations in SES to depressive symptoms path coefficients among groups. Mean level of alcoholic beverage use remained higher for Whites from the first time point to the second time point $(\mathrm{M}=0.40, \mathrm{SE}=0.015$ to $\mathrm{M}=0.41, \mathrm{SE}=0.017)$ whereas mean 
level of alcoholic beverage use decreased for Blacks $(\mathrm{M}=0.22, \mathrm{SE}=0.024$ to $\mathrm{M}=0.20$, $\mathrm{SE}=0.024)$ and Hispanics $(\mathrm{M}=0.24, \mathrm{SE}=0.032$ to $\mathrm{M}=0.18, \mathrm{SE}=0.026)$. Body mass index for all race/ethnic groups remained fairly stable with Blacks having the highest score over time compared to Whites and Hispanics. Paired t-tests indicated that, increase in mean levels of alcoholic beverage use from the first time point to the second time point as well as increase in BMI between adjacent time points, for each race/ethnic group, were not significantly different.

There was slight decrease in mean social integration scores from the first time point to the second time point across all groups. Over time, Blacks had higher mean physical impairment scores indicating possible rapid decline in physical functional status followed by Hispanics. Mean scores of physical impairment for Blacks at the second time point $(\mathrm{M}=0.62, \mathrm{SE}=0.054)$ and at the third time point $(\mathrm{M}=0.91, \mathrm{SE}=0.058)$ were fairly higher than those for Whites at the second time point $(\mathrm{M}=0.29, \mathrm{SE}=0.013)$ and at the third time point $(\mathrm{M}=0.48 ; \mathrm{SE}=0.023)$, but only slightly higher than those for Hispanics at the second time point $(\mathrm{M}=0.56, \mathrm{SE}=0.052)$ and at the third time point $(\mathrm{M}=0.84$, $\mathrm{SE}=0.059)$. Across groups, mean differences of physical impairment scores were significantly higher at the third time point compared to mean scores at the first time point, where for the White group, $\mathrm{t}(4497)=7.06, \mathrm{p}<0.001$, for the Black group, $\mathrm{t}(876)=4.73$, $\mathrm{p}<0.001$, and for the Hispanic group, $\mathrm{t}(517)=3.45, \mathrm{p}<0.001$. Similarly, a paired $\mathrm{t}$-test indicated that mean scores of physical impairment at the third time point compared to mean scores at the second time point in each group were significantly higher in all race/ethnic groups, (White: $\mathrm{t}(3873)=12.16, \mathrm{p}<0.001$; Black: $\mathrm{t}(746)=6.38, \mathrm{p}<0.001$; and 
Hispanic: $\mathrm{t}(443)=5.43, \mathrm{p}<0.001)$. There seemed to be larger increase in mean physical impairment scores for Blacks and Hispanics indicating possible accelerated decline in physical functional status for these groups.

Table 3: Means/proportions for mediating variables and outcome variable at later times

\begin{tabular}{|c|c|c|c|c|}
\hline & $\begin{array}{l}\text { Total } \\
\text { Mean (SE) } / \%\end{array}$ & $\begin{array}{l}\text { White } \\
\text { Mean (SE) } / \%\end{array}$ & $\begin{array}{l}\text { Black } \\
\text { Mean (SE) /\% }\end{array}$ & $\begin{array}{l}\text { Hispanic } \\
\text { Mean (SE) /\% }\end{array}$ \\
\hline \multicolumn{5}{|c|}{ Mediating variables } \\
\hline $\mathrm{SI}_{\mathrm{t}=2}$ & $2.55(0.021)$ & $2.61(0.025)$ & $2.28(0.046)$ & $2.15(0.043)$ \\
\hline $\mathrm{DS}_{\mathrm{t}=2}$ & $0.16(0.004)$ & $0.15(0.004)$ & $0.21(0.009)$ & $0.25(0.014)$ \\
\hline \multicolumn{5}{|c|}{ Behavioral factors } \\
\hline $\mathrm{BMI}_{\mathrm{t}=2}$ & $27.83(0.098)$ & $27.61(0.108)$ & $29.29(0.224)$ & $28.43(0.295)$ \\
\hline $\mathrm{Alc}_{\mathrm{t}=2}$ & $0.37(0.015)$ & $0.41(0.017)$ & $0.20(0.024)$ & $0.181(0.026)$ \\
\hline \multicolumn{5}{|c|}{ Outcome variables } \\
\hline $\mathrm{PI}_{\mathrm{t}=2}$ & $0.30(0.014)$ & $0.29(0.013)$ & $0.62(0.054)$ & $0.56(0.052)$ \\
\hline $\mathrm{PI}_{\mathrm{t}=3}$ & $0.51(0.022)$ & $0.48(0.023)$ & $0.91(0.058)$ & $0.84(0.059)$ \\
\hline $\mathrm{OH}_{\mathrm{t}=2}, \mathrm{n}^{*}$ (prop.) & $1367.06(0.203)$ & $1027.11(0.2050)$ & $227.56(0.213)$ & $108.09(0.175)$ \\
\hline $\mathrm{OH}_{\mathrm{t}=3}, \mathrm{n}^{*}$ (prop.) & $1420.22(0.225)$ & $1075.54(0.226)$ & $237.58(0.246)$ & $99.14(0.180)$ \\
\hline
\end{tabular}

Note: Subscript indicates time; ${ }^{*}$ weighted $\mathrm{n}$ (for overnight hospitalization). SI=social integration, $\mathbf{D S}=$ depressive symptoms, $\mathbf{B M I}=$ body mass index, Alc=alcoholic beverage use, $\& \mathbf{P I}=$ physical impairment, $\mathbf{O H}=$ overnight hospitalization.

At Time 2, proportion of Whites overnight hospitalized was 0.21 and at the third time point, the proportion increased slightly to 0.23 . For Blacks, proportion of overnight hospitalizations at the second time point and third time point were, respectively, 0.21 and 0.25, whereas, proportion of overnight hospitalizations among Hispanics were roughly 0.18 at the second and third time points. These indicate slight increase in the White and 
Black groups but essentially no difference for the Hispanic group in overnight hospitalization over time.

\subsection{Bivariate Relationships among Study Variables}

Tables 4, 5, 6, and 7 present correlations of observed variables for the Total sample, for the White group, for the Black group, and for the Hispanic group respectively. The correlations among the SES variables were significant in each of the race/ethnic groups. However, correlations between income and education as well as between income and wealth appeared to be stronger in the White group compared to correlations of education, income, and wealth in the Black, and Hispanic groups, indicating possible higher return on educational achievement for Whites. As expected, there was a significant negative correlation between each SES variable and physical impairment scores over time in all groups. Similarly, social integration scores were negatively correlated with physical impairment over time, indicating that higher social integration scores were related to lower physical impairment scores in all race/ethnic groups. 


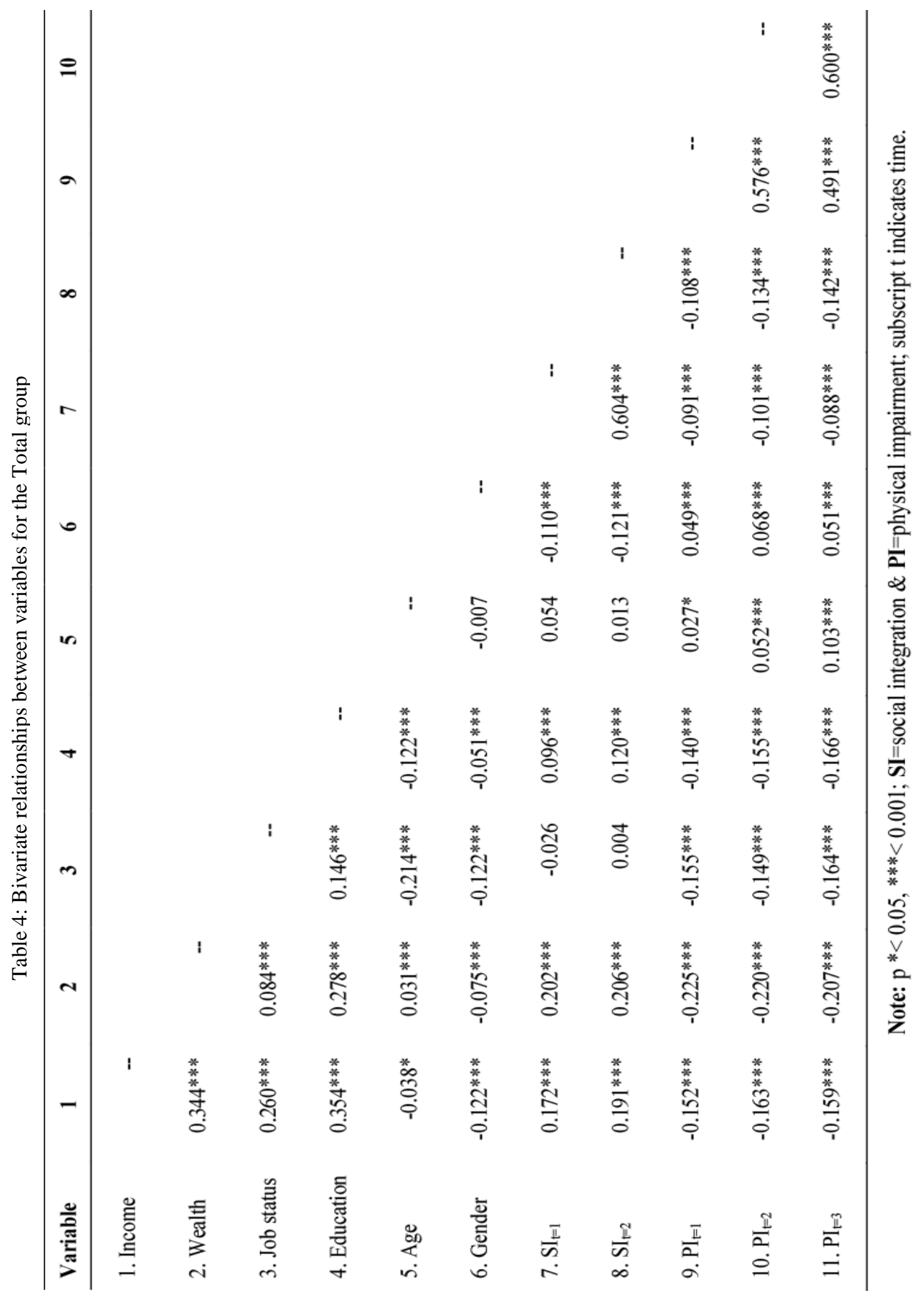




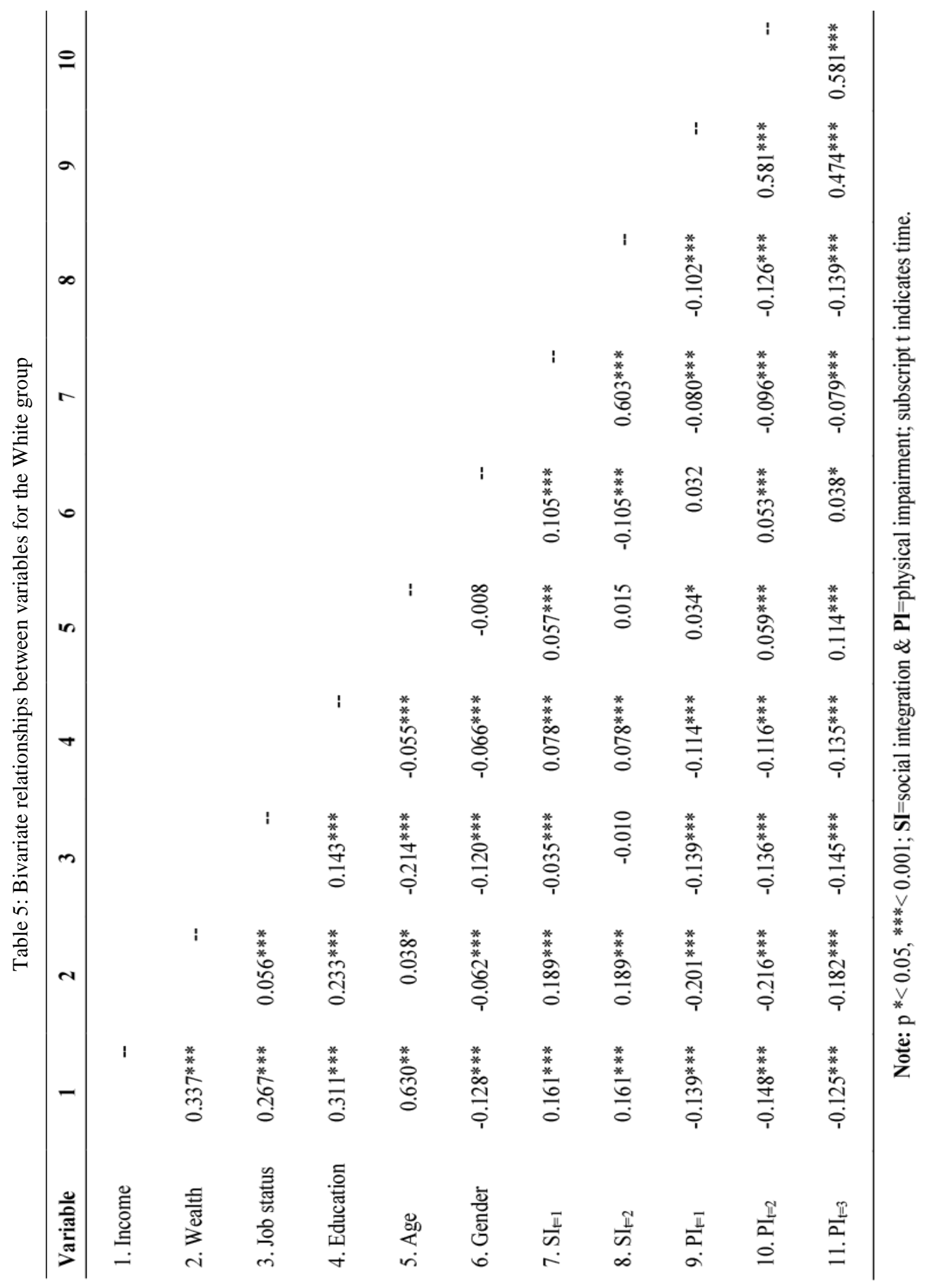




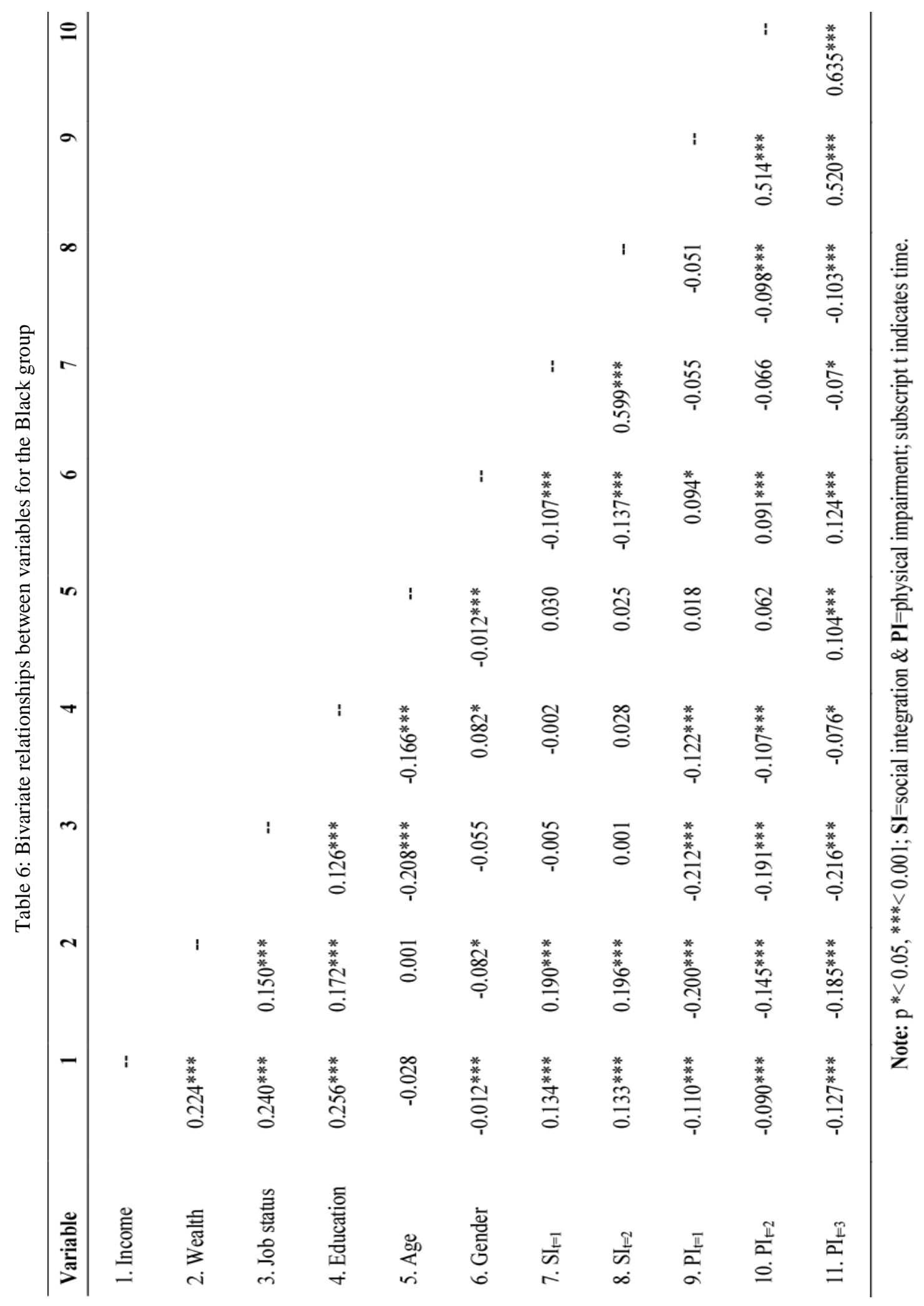




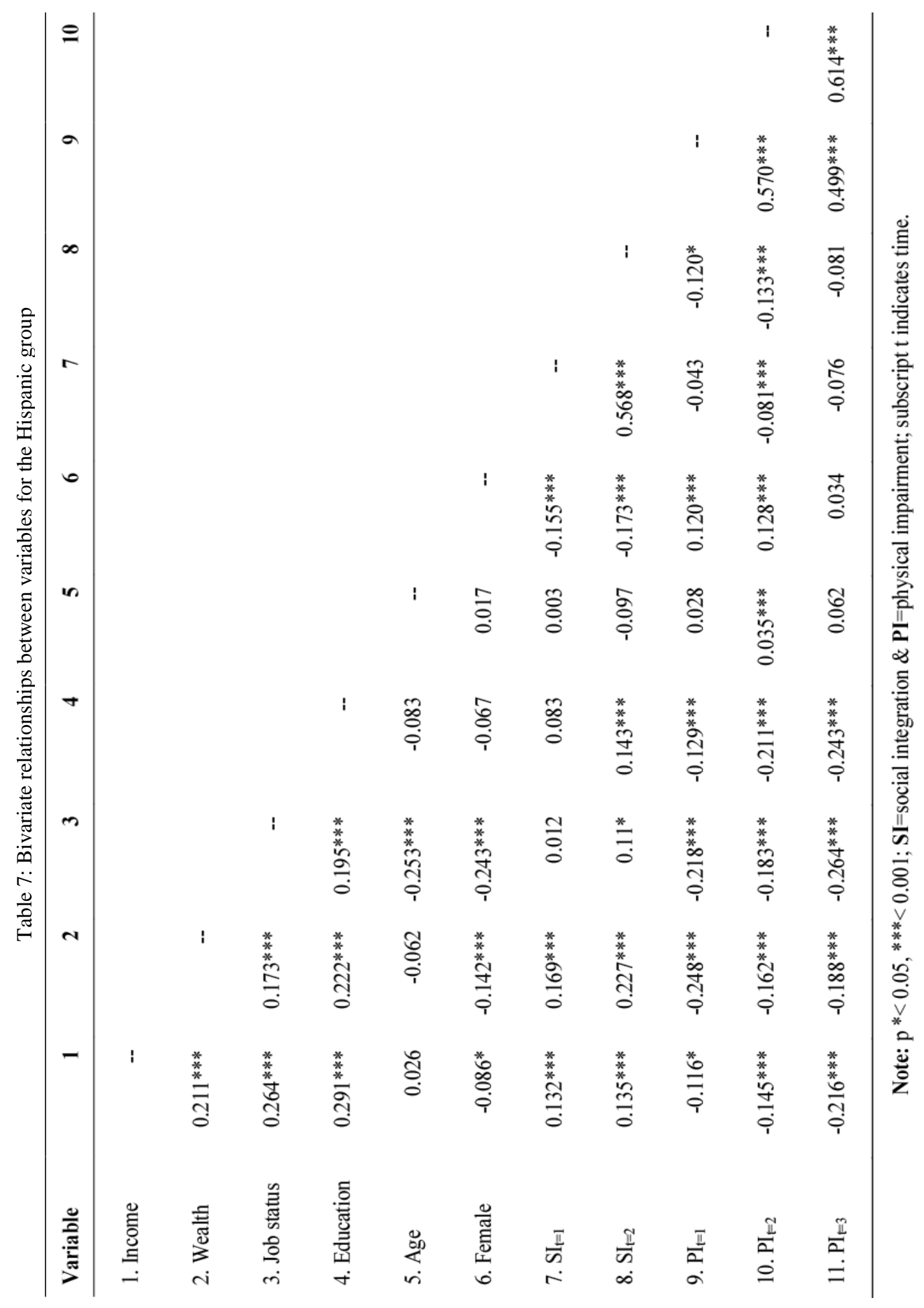


In these analyses, the aim was to examine indirect relationships between SES variables and health outcome variables using lagged path analysis and latent growth curve modeling approaches. Several hypotheses examining the indirect relationships between SES variables and the outcome variables, physical impairment and overnight hospitalization, through the proposed mediating variables were tested in multiple-group framework.

Summary results for the significant indirect effects for the Structural Equation Modeling (SEM) method are provided in the next page. Following the summary presentation, the remaining sections in this chapter present detailed results of the SEM analyses.

\subsection{Summary Results of Significant Indirect Effects for the SEM Method}

Eight SEM models (6 path models and 2 latent growth curve models) were examined in this section and summary of the indirect effects are provided below:

Physical impairment:

- Model 1 (Path model) - Social (S): indirect effects of income \& wealth in the White group were significant.

- Model 2 (Growth curve) - Social (S): indirect effects of education \& job status in the White group were significant

- $\quad$ Model 3 (Path model) - Psychological (P):

- Indirect effects of income, wealth, education \& employment in the White group were significant

- Indirect effects of Wealth \& education in Hispanics were significant

- Model 4 (Growth curve) - Psychological (P): no significant indirect paths

- $\quad$ Model 5 (Path model) - Behavioral (B): 
Indirect effect of wealth on physical impairment through BMI was significant in the White group.

Overnight hospitalization:

- Model 6 (Path model) - Social (S) : no significant indirect paths

- Model 7 (Path model) - Psychological (P): marginal indirect effects of income in the Black group but in opposite direction (inconsistent mediation)

- Model 8 (Path model) - Behavioral (B):

- Indirect effect of wealth on overnight hospitalization through BMI in the White group was significant.

- Indirect effects of income and wealth on overnight hospitalization through drinking were significant in the White group but in the opposite direction (inconsistent mediation).

Effect sizes were small across race/ethnic groups (Table 8) but such findings are not uncommon in race/ethnic studies of indirect effects (Molina, Alegría, \& Mahalingam, 2013) because the analysis was focused on process rather than mean comparison, and because indirect effects were products of coefficients that were small $(<1)$. Note that in Table 8, SI=social integration, $\mathbf{P I}=$ physical impairment, $\mathbf{D S}=$ =depressive symptoms, $\mathbf{B M I}=$ body mass index, $\mathbf{A L C}=$ drinking, $\mathbf{O H}=$ overnight hospitalization. 
Table 8: Summary of significant indirect effects across groups (unstandardized/standardized coefficients). Standard errors are in parentheses

\begin{tabular}{lllll}
\hline & Total sample & White & Black & Hispanic \\
\hline \multicolumn{4}{l}{ Indirect effects of SES on physical impairment via social integration - Path model } \\
Income $\rightarrow \mathrm{SI}_{\mathrm{t}=2} \rightarrow \mathrm{PI}_{\mathrm{t}=3}$ & $-0.003 *(0.001)$ & $-0.003 *(0.001)$ & $-0.001(0.001)$ & $0.002(0.002)$ \\
& -0.003 & -0.003 & -0.001 & 0.002 \\
Wealth $\rightarrow \mathrm{SI}_{\mathrm{t}=2} \rightarrow \mathrm{PI}_{\mathrm{t}=3}$ & $-0.001 *(0.000)$ & $-0.001 *(0.000)$ & $0.000(0.000)$ & $0.001(0.001)$ \\
& -0.003 & -0.003 & -0.002 & 0.004
\end{tabular}

Indirect effects of SES on physical impairment slope factor via social integration slope factorgrowth curve model

$\begin{array}{lllll}\mathrm{Job}_{\mathrm{N}} \rightarrow \mathrm{SI}_{\text {slope }} \rightarrow P I_{\text {slope }} & -0.018^{* * *}(0.002) & -0.007 *(0.002) & 0.002(0.007) & 0.00(0.017) \\ & -0.018 & -0.21 & 0.005 & -0.001 \\ \text { Education } \rightarrow S I_{\text {slope }} \rightarrow \mathrm{PI}_{\text {slope }} & -0.012^{*}(0.000) & -0.001 *(0.000) & -0.001(0.001) & 0.00(0.000) \\ & -0.012 & -0.01 & -0.015 & 0.000\end{array}$

Indirect effects of SES on physical impairment via psychological (path model)

$\begin{array}{lllll}\text { Income } \rightarrow \mathrm{DS}_{\mathrm{t}=2} \rightarrow \mathrm{PI}_{\mathrm{t}=3} & -0.003(0.002) & -0.004 *(0.002) & 0.005(0.007) & -0.007(0.011) \\ & -0.004 & -0.004 & 0.005 & -0.009 \\ & & & \\ \text { Wealth } \rightarrow \mathrm{DS}_{\mathrm{t}=2} \rightarrow \mathrm{PI}_{\mathrm{t}=3} & -0.002 *(0.001) & -0.002 *(0.001) & -0.002(0.002) & -0.005 *(0.002) \\ & -0.008 & -0.007 & -0.006 & -0.021 \\ & -0.01 *(0.003) & -0.008^{*}(0.001) & -0.025(0.004) & -0.007(0.003) \\ \mathrm{Job} \rightarrow \mathrm{DS}_{\mathrm{t}=2} \rightarrow \mathrm{PI}_{\mathrm{t}=3} & -0.008 & -0.003 & -0.008 & -0.002 \\ & -0.003 * *(0.001) & -0.002 *(0.003) & -0.006(0.017) & -0.007 *(0.034) \\ \text { Education } \rightarrow \mathrm{DS}_{\mathrm{t}=2} \rightarrow \mathrm{PI}_{\mathrm{t}=3} & -0.004 & -0.005 & -0.012 & -0.022 \\ & & & \\ \text { Indirect effects of SES on physical impairment via BMI } & -0.0001 *(0.00) & 0.00(0.00) & -0.001(0.001) \\ \text { Wealth } \rightarrow \mathrm{BMI}_{\mathrm{t}=2} \rightarrow \mathrm{PI}_{\mathrm{t}=3} & 0.00(0.00) & -0.001 & 0.00 & -0.004\end{array}$

Indirect effects of SES on overnight hospitalization via drinking and BMI

$\begin{array}{cllll}\text { Income } \rightarrow \mathrm{ALC}_{\mathrm{t}=2} \rightarrow \mathrm{OH}_{\mathrm{t}=3} & 0.001(0.000) & 0.002 *(0.001) & -0.002(0.002) & 0.000(0.001) \\ & 0.001 & 0.002 & -0.002 & 0.000 \\ \text { Wealth } \rightarrow \mathrm{BMI}_{\mathrm{t}=2} \rightarrow \mathrm{OH}_{\mathrm{t}=3} & 0.000(0.000) & 0.000^{*}(0.000) & 0.001(0.000) & 0.000(0.00) \\ & -0.001 & -0.001 & 0.003 & 0.001 \\ \text { Wealth } \rightarrow \mathrm{ALC}_{\mathrm{t}=2} \rightarrow \mathrm{OH}_{\mathrm{t}=3} & 0.000(0.000) & 0.000^{\S}(0.000) & 0.000(0.001) & 0.000(0.002) \\ & 0.000 & 0.001 & 0.000 & 0.000\end{array}$

Note: $\mathrm{p} *<0.05, * * *<0.001,{ }^{\S}$ marginally significant. Only models with significant indirect effects are provided. Subscripts indicate time; SES variables were from the first time point; subscript $t$ indicates time points. Values in parentheses are 


\section{Physical Impairment Status}

The first outcome variable examined was physical impairment status; an index composed of five activities of daily living (ADL) questions. In the following sections, results of lagged path analysis and latent growth curve analysis examining the mediating role of social, psychological and behavioral factors in the SES to physical impairment relationship are provided.

\subsection{SES-Social factors-Physical Impairment: Lagged Path Model}

Hypothesis 1a: Each SES variable will be negatively related to physical impairment but positively related to social integration; social integration, in turn, will be negatively related to physical impairment. These path coefficients will differ across racelethnic groups.

\subsubsection{Path Model for the Total Sample}

A lagged regression path model was fit to the total sample to ensure that the proposed model was appropriate. Results showed that the path model for the entire sample fit to the data well, $\left[\chi^{2}(2)=94.5, \mathrm{CFI}=0.98, \mathrm{SRMR}=0.015\right]$, providing confidence in the model specified. The direct effects of income $\left(\beta_{\text {inc }}=0.059, \mathrm{SE}=0.014, \beta^{*}{ }_{\text {inc }}=0.060, p\right.$ $<0.001)$, wealth $\left(\beta_{\mathrm{wth}}=0.017, \mathrm{SE}=0.003, \beta^{*}{ }_{\text {wlth }}=0.055, \mathrm{p}<0.001\right)$ and education $\left(\beta_{\text {edu }}=0.01, \mathrm{SE}=0.004, \beta^{*}{ }_{\text {edu }}=0.024, \mathrm{p}<0.05\right)$ on social integration at the second time point were significant, indicating increase in SES was related to higher social integration scores consistent with the hypothesis. Path coefficients for the adjusted direct effects of wealth $\left(\beta_{\text {wlth }}=-0.015, \mathrm{SE}=.004, \beta^{*}{ }_{\text {wlth }}=-0.054, \mathrm{p}<0.001\right)$, education $\left(\beta_{\mathrm{edu}}=-0.018\right.$, 
$\left.\mathrm{SE}=0.007, \beta_{\text {edu }}^{*}=-0.045, \mathrm{p}<0.05\right)$ and employment $\left(\beta_{\mathrm{job}}=-0.129, \mathrm{SE}=0.032, \beta_{\mathrm{job}}^{*}=-0.052\right.$, $\mathrm{p}<0.001)$ on physical impairment at the third time point, controlling for the effects of social integration were also significant. Path coefficients pertinent to significant indirect relationships are provided below (Figure 13).

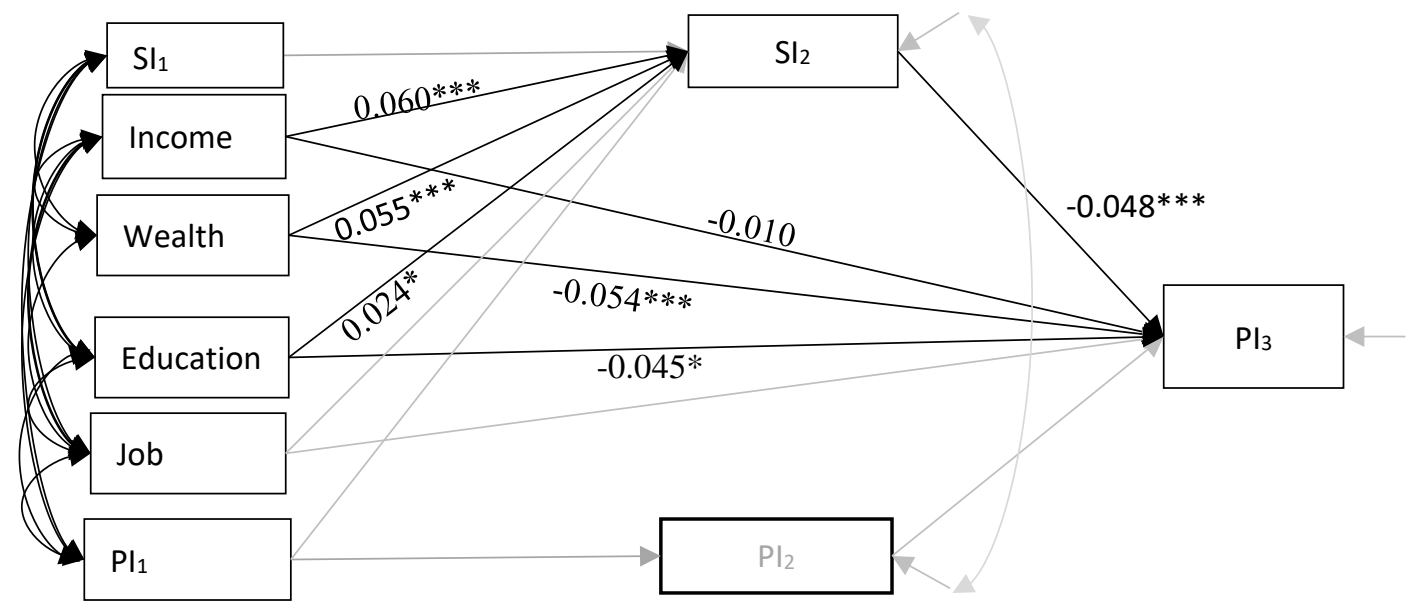

Figure 13: Lagged regression model for Total sample $(\mathrm{N}=7674)$ with standardized path coefficients (Social Integration Mediator)

Note: $* \mathrm{p}<0.05 ; * * * \mathrm{p}<0.001$. Only paths pertinent to significant indirect relationships are shown. All control variables, paths modeled and correlations are not shown to avoid clutter. $\mathbf{S I}=$ =social integration, $\mathbf{P I}=$ physical impairment, and subscripts indicate time points.

The primary aim of the study in this section was to examine indirect associations of each SES variable to physical impairment via social integration. Results showed that three out of the four SES variables, namely income $\left(\beta_{\mathrm{inc}}=-0.003, \mathrm{SE}=0.001, \beta^{*}{ }_{\mathrm{inc}}=-0.003\right.$, $\mathrm{p}<0.05)$, wealth $\left(\beta_{\text {wlth }}=-0.001, \mathrm{SE}=0.0001, \beta^{*}{ }_{\text {wlth }}=-0.003, \mathrm{p}<0.05\right)$ and education $\left(\beta_{\text {edu }}=\right.$ $\left.-0.000, \mathrm{SE}=0.0001, \beta_{\text {edu }}^{*}=0.001, \mathrm{p}<0.05\right)$ were indirectly related to levels of physical impairment. Thus, an increase in income was associated with a small decrease in physical impairment through increase in social integration, controlling for other SES and demographic variables. Further, increase in wealth and education were each related to 
small decrease in physical impairment status through social integration, controlling for all other covariates.

Thus, overall, with the exception of employment status, these results supported the hypothesis that SES variables were indirectly related to physical impairment in the total sample. The model was extended to multiple groups and results are provided below.

\subsubsection{Multiple Group Path Model}

The lagged regression path model tested for the entire group was specified for multiple groups with the objective of evaluating path differences across groups. Based on

alternative fit indices, the multiple group path model fit to the data well, $\left[\left(\chi^{2}(12)=165.97\right.\right.$, $\mathrm{CFI}=0.97, \mathrm{SRMR}=0.017$ ), indicating that the proposed path model was plausible. The next sections provide path coefficients for each race/ethnic group.

\subsubsection{Direct Effects of SES on Social Integration}

I hypothesized that each SES variable will be positively related to social integration such that increase in SES will be related to higher social integration scores, and these higher social integration scores, in turn, will be related to lower physical impairment scores. Results indicated that income and wealth were significantly related to social integration in each race/ethnic group. The effects of income and wealth on social integration at the second time point for the White group, $\left(\beta_{\mathrm{inc}}=0.055, \mathrm{SE}=0.016\right.$, $\left.\beta_{\text {inc }}^{*}=0.053, \mathrm{p}<0.001 ; \beta_{\text {wlth }}=0.013, \mathrm{SE}=0.005, \beta^{*}{ }_{\text {wlth }}=0.041, \mathrm{p}<0.05\right)$, for the Black group $\left(\beta_{\text {inc }}=0.043, \mathrm{SE}=0.022, \beta^{*}{ }_{\text {inc }}=0.052, \mathrm{p}<0.05 ; \beta_{\text {wlth }}=0.015, \mathrm{SE}=0.005, \beta^{*}{ }_{\text {wlth }}=0.074, \mathrm{p}<\right.$ $0.05)$ and for the Hispanic group $\left(\beta_{\text {inc }}=0.034, \mathrm{SE}=0.016, \beta^{*}{ }_{\text {inc }}=0.050, \mathrm{p}<0.05 ; \beta_{\text {wlth }}=\right.$ 
$0.02, \mathrm{SE}=0.009, \beta^{*}{ }_{\text {wth }}=0.093, \mathrm{p}<0.05$ ) were all significant, consistent with the hypothesized relations. Thus, Whites, Blacks and Hispanics with higher incomes and wealth appeared to be more socially integrated. The standardized path coefficients above indicate that increase in income was associated with small increase in social integration across groups and the effect sizes were similar. The association of wealth with social integration across race/ethnic groups seemed to vary, however. Increase in wealth was associated with smaller increase in social integration in Whites while in the Black and Hispanic groups, increase in wealth was associated with larger increase in social integration than the increase observed in the White group. These results were consistent with the hypothesized associations between SES and social integration for all groups although the SES to social integration relationship was expected to be stronger in the White group.

Social integration to physical impairment path coefficients varied across race/ethnic groups. In the White group, social integration predicted physical impairment ( $\left.\beta=-0.057, \mathrm{SE}=0.014, \beta^{*}=-0.062, \mathrm{p}<0.001\right)$, adjusted for the SES and demographic variables. Higher social integration scores were related to lower physical impairment levels in Whites consistent with the hypothesis. In Hispanics and Blacks, the social integration to physical impairment path coefficient was not significant, however.

\subsubsection{Direct Effects of SES on Physical Impairment}

Individuals with higher SES status were expected to have lower physical impairment scores. Each of the SES variables would then be negatively related to 
physical impairment over time such that respondents in higher SES positions would have lower physical impairment levels at successive time points. In the White group, employment status $\left(\beta_{\mathrm{job}}=-0.107, \mathrm{SE}=0.034, \beta_{\text {job }}^{*}=-0.046, \mathrm{p}<0.05\right)$ and education $\left(\beta_{\text {edu }}=-\right.$ $0.022, \mathrm{SE}=0.008, \beta^{*}$ edu $\left.=-0.052, \mathrm{p}<0.05\right)$ were significantly related to physical impairment, indicating that being employed and having higher levels of education were related to lower levels of physical impairment. Wealth was only marginally related to physical impairment $\left(\beta_{\mathrm{wlth}}=-0.014, \mathrm{SE}=0.007, \beta^{*}{ }_{\text {wlth }}=-0.046, \mathrm{p}=0.064\right)$ in the White group. In the Black group, wealth $\left(\beta_{\text {wlth }}=-0.018, \mathrm{SE}=0.008, \beta^{*}{ }_{\text {wlth }}=-0.070, \mathrm{p}<0.05\right)$ and employment status $\left(\beta_{\mathrm{job}}=-0.212, \mathrm{SE}=0.08, \beta^{*}{ }_{\mathrm{job}}=-0.066, \mathrm{p}<0.05\right)$ were significantly related to physical impairment where increase in wealth was related to a moderate decrease in physical impairment and being employed was associated with similar decrease in physical impairment status, controlling for other variables in the model. Similarly, in the Hispanic group, wealth $\left(\beta_{\mathrm{wlth}}=-0.012, \mathrm{SE}=0.006, \beta^{*}{ }_{\text {wlth }}=-0.048, \mathrm{p}<0.05\right)$ and employment status $\left(\beta_{\mathrm{job}}=-0.44, \mathrm{SE}=0.109, \beta^{*}{ }_{\mathrm{job}}=-0.140, \mathrm{p}<0.001\right)$ were significantly related to physical impairment, where increase in wealth was associated with a small decrease in physical impairment scores and being employed was associated with larger decrease in physical impairment.

\subsubsection{Indirect Effects of SES on Physical Impairment}

The main goal of this model was to investigate differences in indirect effects of each of the SES variable on physical impairment via social integration by race/ethnic group. Results varied for the groups, where, only two SES (income and wealth) variables were indirectly related to physical impairment in the White group (Figure 14) and none of 
the indirect relationships for the other groups were significant. The indirect association of income with physical impairment through social integration was significant $\left(\beta_{\mathrm{inc}}=-0.003\right.$, $\left.\mathrm{SE}=0.001, \beta^{*}{ }_{\text {inc }}=-0.003, \mathrm{p}<0.05\right)$ in the White group. This indicated that increase in income was indirectly related to a small decrease in physical impairment through the social integration variable, controlling for other variables in the model. Wealth was also significantly indirectly related to physical impairment in the White group $\left(\beta_{\text {wlth }}=-0.001\right.$, $\left.\mathrm{SE}=0.000, \beta^{*}{ }_{\text {wlth }}=-0.003, \mathrm{p}<0.05\right)$, indicating that wealth had a protective effect from physical impairment through social integration in this group. The standardized path coefficient indicated that increase in wealth was associated with a small decrease in physical impairment via social integration, controlling for other SES/demographic variables. Wealthier Whites appeared to have higher social integration scores which, in turn, seemed to be related to lower physical impairment scores. None of the SES variables were indirectly related to physical impairment scores in the Hispanic or Black groups, however.

Sizes of the indirect effects were small. Overall variances accounted for in the social integration variable at the second time point in the White group (R-square $=0.371$, $\mathrm{SE}=0.017, \mathrm{p}<0.001)$, in the Black group $(\mathrm{R}$-square $=0.379, \mathrm{SE}=0.024, \mathrm{p}<0.001)$ and in the Hispanic group $(\mathrm{R}$-square $=0.368, \mathrm{SE}=0.030, \mathrm{p}<0.001)$ were not trivial, however. Similarly variances accounted for in physical impairment at the third time point in the White group $(\mathrm{R}$-square $=0.353, \mathrm{SE}=0.028, \mathrm{p}<0.001)$, Black group $(\mathrm{R}$-square $=0.425$, $\mathrm{SE}=0.056, \mathrm{p}<0.001)$ and Hispanic group $(\mathrm{R}$-square $=0.420, \mathrm{SE}=0.069, \mathrm{p}<0.001)$ were substantial. R-square values for the physical impairment variable, at the third time point, 
in all the models that follow were similar to those presented here and were excluded from results in subsequent sections.

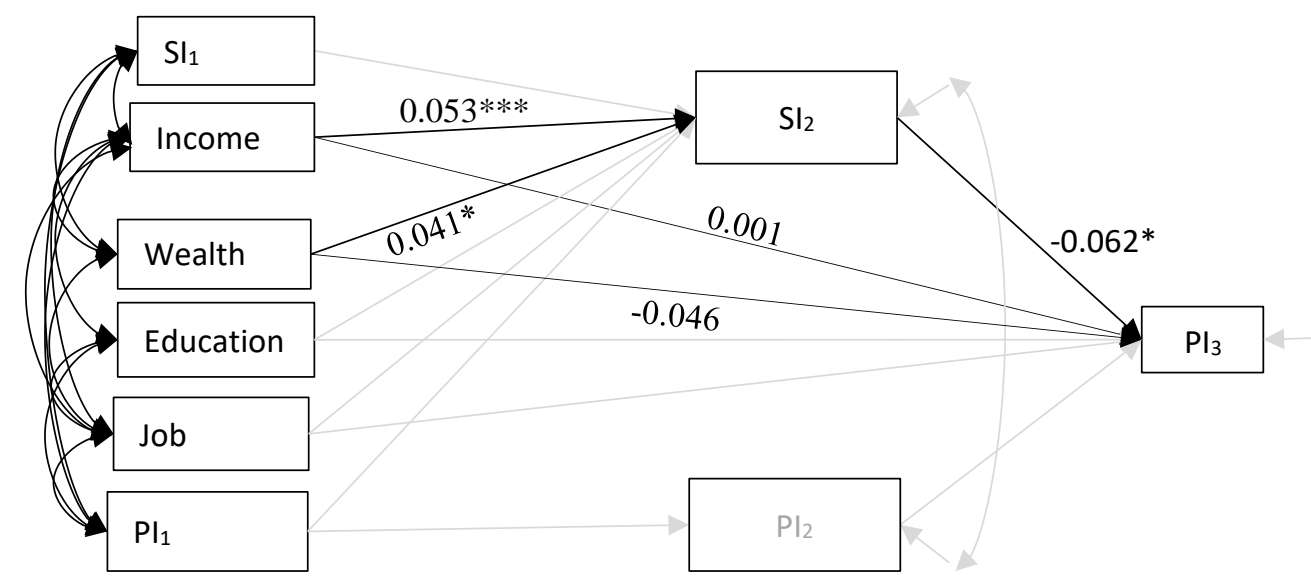

Figure 14: Lagged regression model for Whites $(\mathrm{N}=5717)$ with standardized path coefficients (Social Integration Mediator)

Note: $* \mathrm{p}<0.05 ; * * * \mathrm{p}<0.001$. Only paths pertinent to significant indirect relationships are shown. All control variables, paths modeled and correlations are not shown to avoid clutter. $\mathbf{S I}=$ =social integration, $\mathbf{P I}=$ physical impairment, and subscripts indicate time points.

To further examine the significant indirect relationships observed in the White group compared to the other groups, I conducted a follow-up analysis using chi-square test of nested models and provide results in the next section.

\subsubsection{Follow-up Analysis of Significant Indirect Paths}

A chi-square test of nested models where SES to social integration, SES to physical impairment, and social integration to physical impairment paths in the null model were constrained across groups was compared with a model where these paths were freely estimated in a multiple group framework. Constraining these paths did not result in significantly poorer fit, $\left(\Delta \chi^{2}(10)=11.94, \mathrm{~ns}\right)$, signaling that the observed differences in path coefficients were not different across groups. 
To further examine group differences in specific indirect paths, I conducted a chisquare test of nested models where specific paths were constrained in the nested model. First, I constrained direct and indirect paths from wealth to physical impairment and examined differences in the constrained and unconstrained models. The constrained and freely estimated models were not significantly different, $\left(\Delta \chi^{2}(6)=5.46\right.$, ns $)$, indicating that indirect association of wealth with physical impairment across groups was invariant. Next, the direct and indirect paths from income to physical impairment were constrained in the nested model. Chi-square results showed that constraining paths from income to physical impairment did not significantly worsen fit as indicated by the nonsignificant chi-square value, $\left(\Delta \chi^{2}(6)=10.02, \mathrm{~ns}\right)$.

\section{Summary}

In summary, although direction of the relationships (correlational relationships of the variables) between SES and social integration, and between social integration and physical impairment were consistent with the hypothesis, only in the White group did income and wealth appear to be indirectly (significantly) related to physical impairment. Follow-up analyses using nested chi-square tests revealed that there were not significant differences in the indirect effects across groups, however.

The next section provides analyses of indirect relationships between SES and physical impairment via social integration using latent growth curve mediation modeling approach. 


\subsection{SES-Social Factors-Physical Impairment: Growth Curve Model}

Hypothesis 1b: Each SES variable will be negatively related to the slope factor for physical impairment but positively related to the slope factor for social integration. The slope factor for social integration, in turn, will be negatively related to the slope factor for physical impairment. These paths will differ across racelethnic groups.

Latent growth curve modeling is one approach to examining mediated effects when the mediator and outcome variables are measured at several time points. Lagged path models do not explicitly model individual differences in the trajectory over time (MacKinnon, 2008). In order to examine the relationship of each SES variable to the trajectory of social integration and the trajectory of physical impairment over time and to examine the relationship of the growth of social integration to the growth of physical impairment, I used latent growth curve mediation modeling approach. In hypothesis $1 \mathrm{~b}$, the trajectory for social integration was expected to predict the trajectory for physical impairment status indicating that higher scores on social integration would be associated with lower physical impairment scores over time. Demographic and SES variables were included as time invariant covariates.

\subsubsection{Latent Growth Curve Model for the Total Sample}

A latent growth curve mediation model was tested to examine whether SES was indirectly related to the trajectory of physical impairment via the growth process for social integration in the total sample. The overall model fit to the date well, $\left[\left(\chi^{2}(77)=227.69, \mathrm{CFI}=0.99, \mathrm{SRMR}=0.014\right)\right]$. Growth factors for social integration and 
physical impairment appeared to reflect the general trend of these two factors in older adults. The social integration slope factor for the total sample in this study was negative $\left(\alpha=-0.124, \mathrm{SE}=0.035, \alpha^{*}=-0.644, \mathrm{p}<0.001\right)$, indicating a general decline of social integration scores over time, whereas, the slope factor for physical impairment was positive $\left(\alpha=0.203, \mathrm{SE}=0.0340, \alpha^{*}=1.080, \mathrm{p}<0.001\right)$ indicating increase in physical impairment over time. Education $\left(\beta_{\text {edu }}=0.005, \mathrm{SE}=0.001, \beta^{*}\right.$ edu $\left.=0.071, \mathrm{p}<0.001\right)$ and job status $\left(\beta_{\mathrm{job}}=0.042, \mathrm{SE}=0.009, \beta_{\mathrm{job}}^{*}=0.106, \mathrm{p}<0.001\right)$ were significantly related to the slope factor for social integration indicating increase in education was related to a moderate increase in the social integration slope factor. Similarly, being employed was related to relatively large increase in the slope factor for social integration. Likewise, the effect of the social integration slope factor on the physical impairment slope factor was significant $\left(\beta=-0.168, \mathrm{SE}=0.035, \beta^{*}=-0.173, \mathrm{p}<0.001\right)$ indicating increase in the slope factor for social integration was associated with sizable decrease in the slope factor for physical impairment.

In the total sample, education $\left(\beta_{\text {edu }}=-0.001, \mathrm{SE}=0.000, \beta^{*}\right.$ edu $\left.=-0.012, \mathrm{p}<0.05\right)$ and employment status $\left(\beta_{\mathrm{job}}=-0.007, \mathrm{SE}=0.002, \beta_{\mathrm{job}}{ }=-0.018, \mathrm{p}<0.001\right)$ were indirectly related to the trajectory of physical impairment via the growth process for social integration. Increase in education level was associated with a small decline in physical impairment through the growth process in social integration. Being employed was also indirectly related to a small decline in physical impairment through social integration. Income and wealth were not indirectly related to physical impairment. 


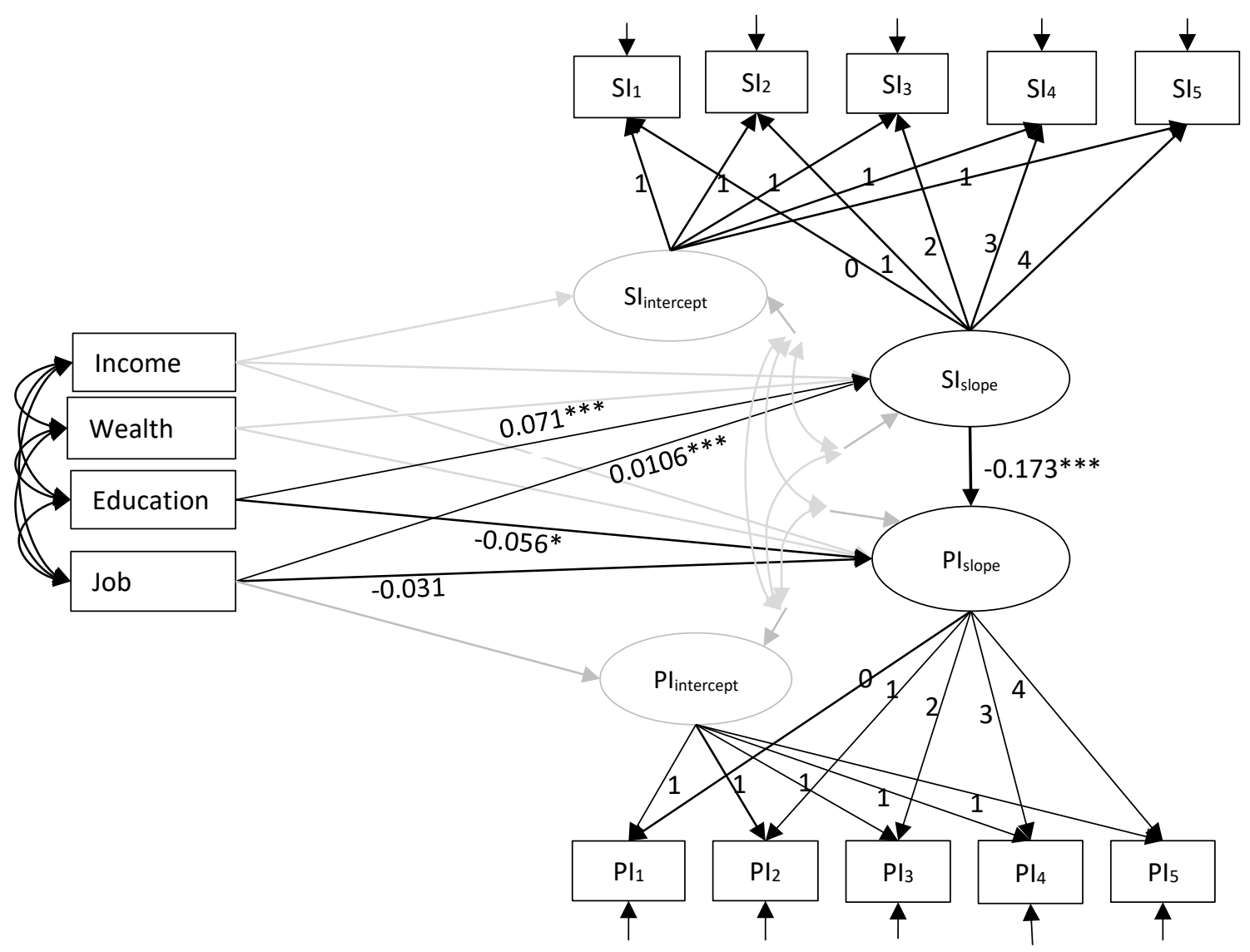

Figure 15: Latent growth curve mediation model for Total sample $(\mathrm{N}=7674)$ with standardized path coefficients (Social Integration Mediator)

Note: $* \mathrm{p}<0.05 ; * * * \mathrm{p}<0.001$. Only paths pertinent to significant indirect relationships are shown. All control variables, paths modeled and correlations are not shown to avoid clutter. SI=social integration, $\mathbf{P I}=$ physical impairment, and subscripts indicate time point. Effects of Age and Gender are not shown in Figure 16; subscripts indicate time points.

The next models consider multiple-group latent growth curve models and examine indirect effects of SES on physical impairment via the trajectory of social integration.

\subsubsection{Growth Curve Model for Individual Race/Ethnic Groups}

The hypothesized latent growth curve model was first tested separately in each group to ascertain plausibility of the model for each group and make specification adjustments until good fitting latent growth curve models to the data for each group were 
obtained. A multiple group model was, then, specified to simultaneously examine direct and indirect path coefficients across race/ethnic groups.

Growth curve modeling involving multiple groups requires that individual group models fit to the data adequately. In order to ensure that the model fit to the data well in each group, I specified and examined a growth curve model for each group before running a multiple group model. The separate models, namely the White group model $\left[\left(\chi^{2}(77)=183.8, \mathrm{CFI}=0.99, \mathrm{SRMR}=0.015\right)\right]$, the Black group model $\left[\left(\chi^{2}(77)=79.1\right.\right.$, $\mathrm{CFI}=0.99, \mathrm{SRMR}=0.018)]$ and the Hispanic group model $\left[\left(\chi^{2}(77)=123.4, \mathrm{CFI}=0.98\right.\right.$, SRMR=0.027)], fit to the data well. Given the above well-fitting total and individual group models, the next sections provide results for multiple group analyses.

\subsubsection{Multiple Group Growth Curve Model for Social Integration and Physical Impairment}

Multiple group growth curve analysis allows for simultaneous assessment of variation in growth factors as well as estimation of direct and indirect path coefficients across groups. The configural structural model fit to the data well, $\left[\left(\chi^{2}(231)=400.3\right.\right.$, $\mathrm{CFI}=0.99, \mathrm{SRMR}=0.017)]$ and growth factors varied across groups. The mean slope factor for social integration in the White group $\left(\alpha_{\mathrm{w}}=-0.119, \mathrm{SE}=0.052, \alpha^{*}{ }_{\mathrm{w}}=-0.611\right.$, $\mathrm{p}<0.05)$ was significant, indicating that social integration, on average, declined every two years in this group. In the Black group, the mean slope factor was also negative but only marginally significant $\left(\alpha_{b}=-0.118, \mathrm{SE}=0.063, \alpha^{*}{ }_{b}=-0.694, p=0.58\right)$, showing that on average, social integration declined every two years for this group. The standardized 
mean values indicated that the size of the mean slope factors for social integration in the White and the Black group were similar. However, the mean slope factor for social integration in the Hispanic group was substantially smaller than that in Whites and Blacks and was not significantly different from zero $\left(\alpha_{h}=-.019, \mathrm{SE}=0.063, \alpha^{*}{ }_{\mathrm{h}}=-0.102\right.$, ns).

Physical impairment increased over time for all race/ethnic groups. The mean slope factor for physical impairment in the White group was significant $\left(\alpha_{w}=0.173\right.$, $\left.\mathrm{SE}=0.051, \alpha^{*}{ }_{\mathrm{w}}=0.989, \mathrm{p}<0.001\right)$, indicating that physical impairment scores in this group, on average, increased every two years. The slope factors for physical impairment in the Black group $\left(\alpha_{b}=0.178, S E=0.082, \alpha^{*}=0.707, p<0.05\right)$ and in the Hispanic group $\left(\alpha_{h}=0.294, S E=0.114, \alpha^{*}{ }_{h}=1.335, p<0.05\right)$ were each significantly different from zero, demonstrating that physical impairment, on average, increased for Blacks and for Hispanics every two years. Whites and Blacks appeared to have similar trajectory of physical impairment over time while the growth rate in Hispanics seemed to be larger than that in Whites and Blacks. The next sections examine direct and indirect path coefficients.

\subsubsection{Direct Effects of SES on the Slope Factor for Social Integration}

Higher income, greater wealth, higher education and being employed are related to higher levels of social integration. Individuals in higher SES positions were, then, expected to have higher social integration scores. Consistent with the above proposition, in Whites, education $\left(\beta_{\text {edu }}=0.004, \mathrm{SE}=0.002, \beta^{*}{ }_{\text {edu }}=0.056, \mathrm{p}<0.05\right)$ and employment 
$\left(\beta_{\mathrm{job}}=0.046, \mathrm{SE}=0.01, \beta_{\mathrm{job}}^{*}=0.114, \mathrm{p}<0.001\right)$ were associated with higher slope values for social integration, indicating that those employed and those with higher levels of education tended to have higher levels of social integration over time. In the Hispanic group, employment $\left(\beta_{\mathrm{job}}=0.096, \mathrm{SE}=0.03, \beta^{*}{ }_{\mathrm{job}}=0.240, \mathrm{p}<0.001\right)$, was related to higher slope values for social integration, indicating that employed Hispanics tended to have higher social integration scores over time. Employment status was the only common variable in the White and Hispanic groups that was significantly related to the social integration slope factor but the effect appeared to be larger in the Hispanic group. This may signal that being employed may be relatively more beneficial to Hispanics in terms of greater integration. None of the SES variables were significantly related to the social integration slope factor in the Black group, failing to support the hypothesized relationships.

The slope factor for social integration predicted the slope factor for physical impairment in the White group, $\left(\beta=-0.163, \mathrm{SE}=0.033, \beta^{*}=-0.182, \mathrm{p}<0.001\right)$, indicating that changes in social integration scores over time were related to changes in physical impairment scores, which, in this case, indicated increase in the slope factor for social integration was associated with moderate decrease in the slope factor for physical impairment every two years. In the Black group, the social integration slope factor marginally predicted the physical impairment slope factor, $\left(\beta=-0.302, \mathrm{SE}=0.16, \beta^{*}=-\right.$ $0.203, \mathrm{p}=0.59)$, indicating that on average, increase in the slope factor for social integration was related to a moderate decrease in the slope factor for physical impairment. Change in the slope factor for social integration was not significantly 
associated with change in the slope factor for physical impairment in the Hispanic group, however.

\subsubsection{Direct Effects of SES on the Slope Factor for Physical Impairment}

The relationship between each of the SES variables and physical impairment was expected to be negative, in that, increase in SES would be associated with decrease in physical impairment. The direct effect of wealth $\left(\beta_{\text {wlth }}=-0.05, \mathrm{SE}=0.002, \beta^{*}{ }_{\text {wlth }}=-0.109\right.$, $\mathrm{p}<0.05)$ on the slope factor for physical impairment in the White group was significant indicating that increase in wealth was associated with decrease in physical impairment, adjusting for other SES and demographic variables. In the Hispanic group, employment $\left(\beta_{\mathrm{job}}=-0.05, \mathrm{SE}=0.023, \beta_{\mathrm{job}}^{*}=-0.105, \mathrm{p}<0.05\right)$ and education $\left(\beta_{\mathrm{edu}}=-0.01, \mathrm{SE}=0.004\right.$, $\left.\beta_{\text {edu }}^{*}=-0.203, p<0.05\right)$ were significantly related to the slope factor for physical impairment while none of the SES variables were significantly related to the slope factor for physical impairment in Blacks. In Hispanics, therefore, increase in job status was associated with relatively moderate decrease in physical impairment and increase in education level was associated with relatively large decrease in physical impairment, controlling for other SES and demographic variables.

\subsubsection{Indirect Effects of SES on the Slope Factor for Physical Impairment}

The central assumption of this study was that each of the SES variables indirectly influenced physical impairment and that this influence differed by race/ethnicity. To examine the hypothesis that higher SES was associated with higher levels of social integration and higher integration, in turn, was associated with lower physical 
impairment, thus investigate whether these paths differed by race/ethnicity, I tested the indirect effect of each SES variable on the trajectory of physical impairment status via the growth factor in social integration. Results showed that the indirect effect of education $\left(\beta_{\text {edu }}=-0.001, \mathrm{SE}=0.00, \beta^{*}{ }_{\text {edu }}=-0.01, \mathrm{p}<0.05\right)$ on the slope factor for physical impairment, via growth in the social integration slope factor, was significant in the White group, indicating that, on average, increase in education was related to a small decline in physical impairment through social integration, controlling for other covariates in the model. Additionally, employment status was indirectly related to the trajectory of physical impairment in Whites $\left(\beta=-0.007, \mathrm{SE}=0.002, \beta^{*}=-0.021, \mathrm{p}<0.001\right)$, where being employed was related to a small decrease in physical impairment via increase in the social integration growth factor. This suggested that Whites who were employed tended to have higher social integration which in turn appeared to be related to lower physical impairment, adjusted for other covariates. 


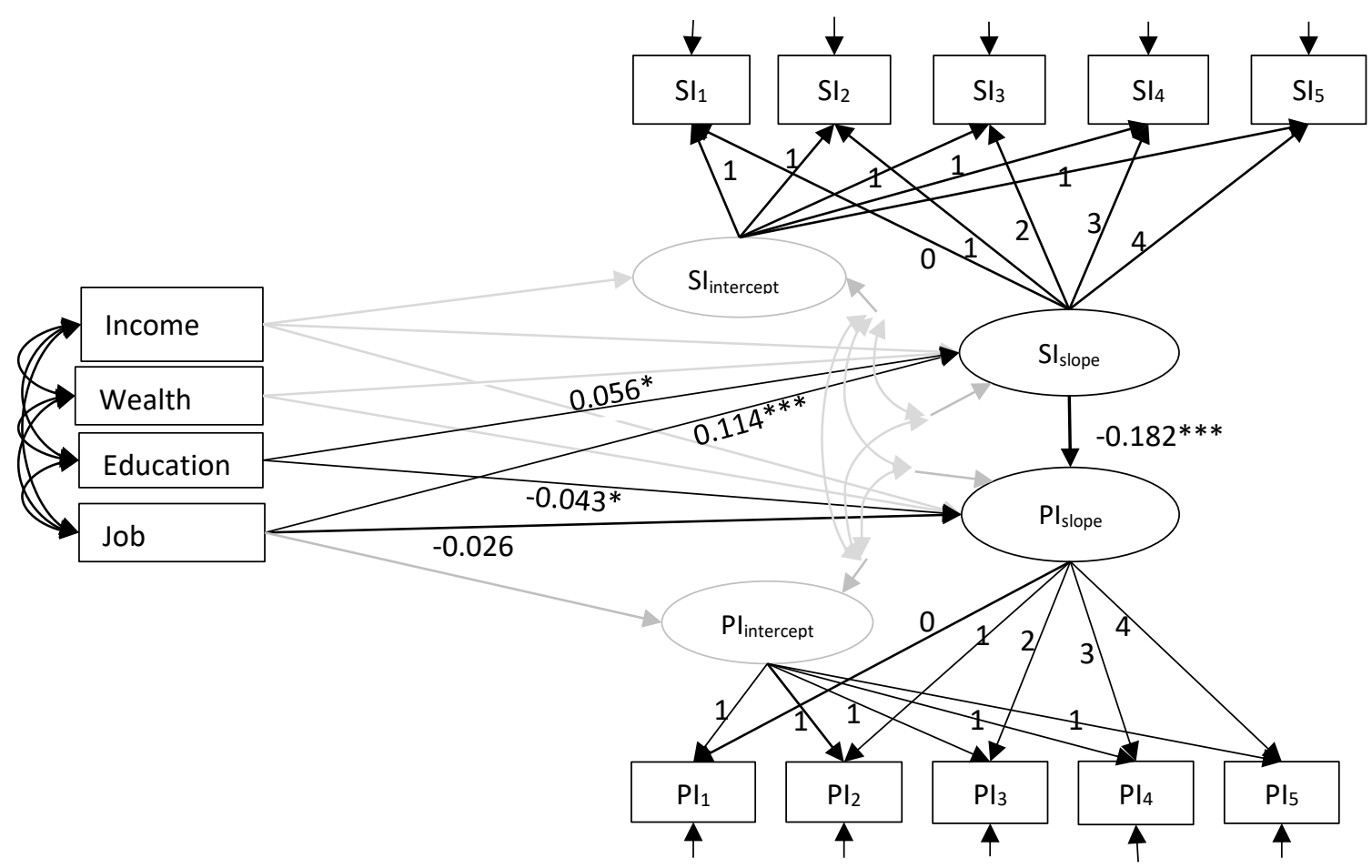

Figure 16: Latent growth curve model for Whites $(\mathrm{N}=5717)$ with standardized path coefficients (Social Integration Mediator)

Note: $* \mathrm{p}<0.05 ; * * * \mathrm{p}<0.001$. Only paths pertinent to significant indirect relationships are shown. All control variables, paths modeled, variances and covariances are not shown for simplicity of presentation. $\boldsymbol{S I}=$ social integration, $\boldsymbol{P I}=$ physical impairment, and subscripts indicate time points.

For Black and Hispanic groups, none of the indirect effects of SES variables on physical impairment through the slope factor for social integration were significant. It is also imperative to note that, while none of the SES to social integration paths were significant in the Black group, the social integration to physical impairment path was only marginally significant. In the Hispanic group, the opposite of the pattern in Blacks was observed where some SES variables predicted the slope factor for social integration but the slope factor for social integration was not associated with the slope factor for physical impairment. 


\subsubsection{Follow-up Analyses}

In order to test if the detected path differences in the above results among the groups were significant, I conducted a series of nested chi-square tests where all paths from SES to social integration slope factor and to physical impairment slope factor, as well as social integration to physical impairment slope factor were constrained equal across groups in the null model and freely estimated in the alternative model. Results indicated that constraining these paths across groups led to a marginal worsening of fit, $\left(\chi^{2}(18)=27.84, p=0.065\right)$, indicating the differences in path coefficients were marginally significantly different across groups. Given this marginal worsening of fit, I conducted further analysis of nested models constraining direct and indirect paths from education to physical impairment slope factor first, and then only constraining direct and indirect paths from job status to physical impairment slope factor. Comparison of the nested model, where, education to physical impairment slope factor paths were constrained across groups to one freely estimated these paths, showed that the models were not significantly different, $\left(\Delta \chi^{2}(237)=405.6, \mathrm{~ns}\right)$, indicating that the indirect effect of education on physical impairment did not significantly vary by race/ethnicity. However, chi-square difference test of the nested models, where, paths from job status to physical impairment slope factor were constrained across groups, resulted in significant worsening of fit, $\left(\Delta \chi^{2}(237)=412.5, \mathrm{p}<0.05\right)$. This suggested that the indirect effect of employment status on the physical impairment slope factor via social integration slope factor, varied by race/ethnicity. 


\section{Summary}

In summary, the direct and indirect associations of SES to physical impairment exhibited slight variation across groups that only involved few paths. Just two SES variables, employment status and education, in the White group were indirectly associated with physical impairment slope factor while none of the SES variables in the Black and Hispanic groups were indirectly associated with physical impairment. These results support the hypothesis that higher SES is indirectly related to lower physical impairment. Follow-up analyses with chi-square tests of nested models showed that the indirect association of education to physical impairment did not significantly differ across groups. However, nested chi-square analysis of models testing invariance of the employment to physical impairment via social integration indicated that these paths were not across groups.

\subsection{SES-Psychological Factors-Physical Impairment: Lagged Path Model}

Hypothesis 2a: Each SES variable will be negatively related to depressive symptoms and physical impairment scores; depressive symptoms, in turn, will be positively related to physical impairment. These paths will differ across racelethnic groups.

\section{Lagged Regression Path Model}

To investigate hypothesis $2 \mathrm{a}$, a lagged regression path analysis approach was used to examine whether each SES variable was indirectly related to physical impairment scores via depressive symptoms and whether these paths differed by race/ethnicity. 


\subsubsection{Path Model for the Total Sample}

A lagged path model was first fit to the total sample to ascertain that the model specified was suitable for this data. The model fit to the data well, $\left[\left(\chi^{2}(47)=502.38\right.\right.$, $\mathrm{CFI}=0.96, \mathrm{SRMR}=0.032)$ ], providing reasonable ground for testing the model in multiple group framework. Regarding path coefficients in the total sample, SES variables were negatively related to physical impairment scores as hypothesized but only the adjusted wealth $\left(\beta_{\text {wlth }}=-0.012, \mathrm{SE}=0.005, \beta^{*}{ }_{\text {wlth }}=-0.045, \mathrm{p}<0.05\right)$ and employment status $\left(\beta_{\mathrm{job}}=-\right.$ $\left.0.099, \mathrm{SE}=0.031, \beta^{*}{ }_{\mathrm{job}}=-0.04, \mathrm{p}<0.05\right)$ path coefficients remained significant. The direct effects of wealth $\left(\beta_{\mathrm{wlth}}=-0.003, \mathrm{SE}=0.001, \beta^{*}{ }_{\text {wlth }}=-0.069, \mathrm{p}<0.001\right)$, employment status $\left(\beta_{\mathrm{job}}=-0.014, \mathrm{SE}=0.005, \beta_{\mathrm{job}}^{*}=-0.034, \mathrm{p}<0.05\right)$ and education $\left(\beta_{\mathrm{edu}}=-0.005, \mathrm{SE}=0.001\right.$, $\left.\beta_{\text {edu }}^{*}=-0.07, p<0.001\right)$ on the depressive symptoms latent variable were significant, providing evidence in support of the hypothesized relationships. The depressive symptoms latent variable at the second time point was significantly (positively) related to physical impairment scores at the third time point, $\left(\beta=0.675, \mathrm{SE}=0.121, \beta^{*}=0.114\right.$, $\mathrm{p}<0.001$ ), controlling for other SES and demographic variables, consistent with the hypothesis in this section.

The main thrust of the study in this section was to examine the indirect relationship between each SES variable and physical impairment scores via depressive symptoms across race/ethnic groups, but I first examined the indirect paths for the total sample. Most of the indirect SES to physical impairment paths including wealth $\left(\beta_{\mathrm{wlth}}=-\right.$ $\left.0.002, \mathrm{SE}=0.001, \beta^{*}{ }_{\text {wlth }}=-0.008, \mathrm{p}<0.05\right)$, employment status $\left(\beta_{\mathrm{job}}=-0.01, \mathrm{SE}=0.003\right.$, $\left.\beta_{\text {job }}^{*}=-0.004, p<0.05\right)$, and education $\left(\beta_{\text {edu }}=-0.003, S E=0.001, \beta_{\text {edu }}^{*}=-0.008, p<0.001\right)$ 
were significant in the total sample lending support to the general idea of indirect link between SES and physical impairment through psychological factors in the total sample over time.

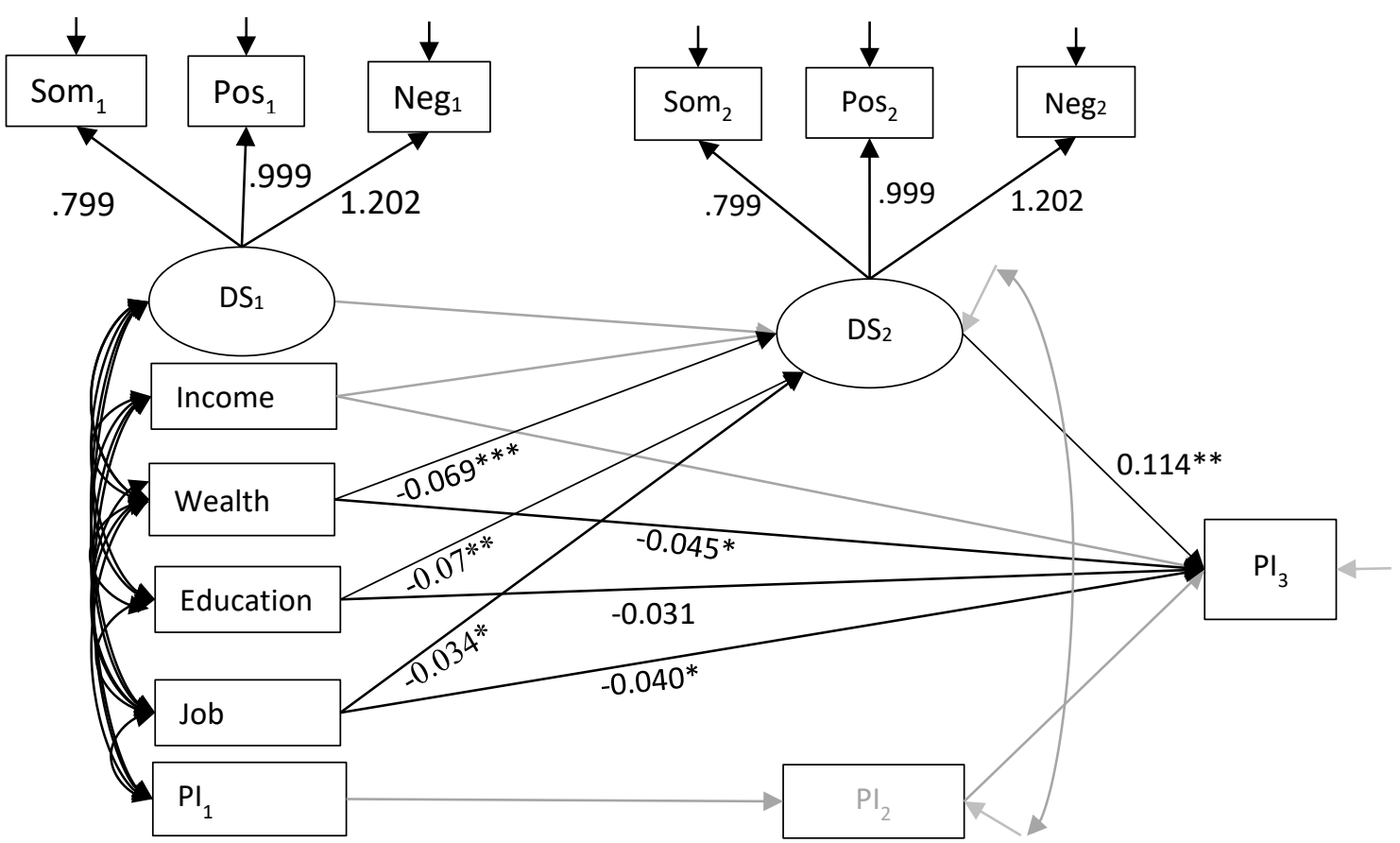

Figure 17: Lagged regression model for Total sample $(\mathrm{N}=7674)$ with standardized path coefficients (Depressive Symptoms Mediator)

Note: $* \mathrm{p}<0.05 ; * * * \mathrm{p}<0.001$. Only paths pertinent to significant indirect relationships are shown. All control variables, paths modeled, variances and covariances are not shown to avoid clutter. $\mathbf{D S}=$ depressive symptoms, $\mathbf{P I}=$ physical impairment, $\mathbf{S o m}=$ somatic symptoms, $\mathbf{P o s}=$ positive affect (reverse coded), Neg=negative affect, and subscripts indicate time points.

\subsubsection{Multiple Group Path Model}

In order to ensure that the lagged path model had a good fit in each group before extending the model to multiple groups, I examined model fit to the data for each group. The model fit to the data adequately for Whites $\left[\left(\chi^{2}(47)=414.59, \mathrm{CFI}=0.95\right.\right.$, SRMR=0.036) , for Blacks $\left[\left(\chi^{2}(47)=135.958, C F I=0.95, S R M R=0.031\right)\right]$, and for Hispanics $\left[\left(\chi^{2}(47)=102.45, \mathrm{CFI}=0.95, \mathrm{SRMR}=0.031\right)\right]$, providing confidence that the 
specified model was reasonable for each group. This model was, then, used in multiple group mediation analysis. Results for the multiple group model indicated that the model had adequate fit to the data, $\left[\left(\chi^{2}(149)=816.12, \mathrm{CFI}=0.94, \mathrm{SRMR}=0.038\right)\right]$, albeit slightly worse than the fit for each of the individual race/ethnic group models. Given this wellfitting model, I examine and provide direct and indirect path coefficients for each race/ethnic group in the next sections.

\subsubsection{Direct Effects of SES on Depressive Symptoms}

Each SES variable was expected to be negatively related to depressive symptoms, where, individuals with higher income, wealth, education and those who were employed would have lower depressive symptoms scores. In the White group, the direct effect of income on depressive symptoms was significant $\left(\beta_{\mathrm{inc}}=-0.007, \mathrm{SE}=0.003, \beta_{\text {inc }}^{*}=-0.041\right.$, $\mathrm{p}<0.05)$, an increase in income, on average, was associated with a small decrease in depressive symptoms. Wealth had similar relationship to depressive symptoms, $\left(\beta_{\mathrm{wlth}}=-\right.$ $\left.0.003, \mathrm{SE}=0.001, \beta^{*}{ }_{\text {wlth }}=-0.068, \mathrm{p}<0.001\right)$, indicating a small decrease in depressive symptoms as wealth increased in the White group. Additionally, job status $\left(\beta_{\mathrm{job}}=-0.013\right.$, $\left.\mathrm{SE}=0.005, \beta_{\text {job }}^{*}=-0.033, \mathrm{p}<0.05\right)$ and education $\left(\beta_{\text {edu }}=-0.004, \mathrm{SE}=0.001, \beta_{\text {edu }}^{*}=-0.05\right.$, $\mathrm{p}<0.05)$ were significantly related to depressive symptoms in the White group, where increase in education was associated with a small decrease in depressive symptoms and being employed was associated with lower depressive symptoms scores.

In Blacks, only job status $\left(\beta_{\text {job }}=-0.028, \mathrm{SE}=0.012, \beta^{*}{ }_{\text {job }}=-0.063, \mathrm{p}<0.05\right)$ and education $\left(\beta_{\text {edu }}=-0.006, \mathrm{SE}=0.002, \beta^{*}\right.$ edu $\left.=-0.093, \mathrm{p}<0.05\right)$ were significantly related to 
depressive symptoms. An increase in education level was associated with relatively large decrease in depressive symptoms and being employed was related to a moderate decrease in depressive symptoms in the Black group. In the Hispanic group, wealth $\left(\beta_{\mathrm{wlth}}=-0.004\right.$, $\left.\mathrm{SE}=0.002, \beta^{*}{ }_{\text {wlth }}=-0.1, \mathrm{p}<0.05\right)$ and educational level $\left(\beta_{\text {edu }}=-0.006, \mathrm{SE}=0.003, \beta^{*}{ }_{\text {edu }}=-\right.$ $0.101, \mathrm{p}<0.05)$ were significantly associated with depressive symptoms, indicating that education and wealth had similar effects on depressive symptoms in the Hispanic group in the other groups. Increase in education level and wealth were each related to relatively large decrease in depressive symptoms scores.

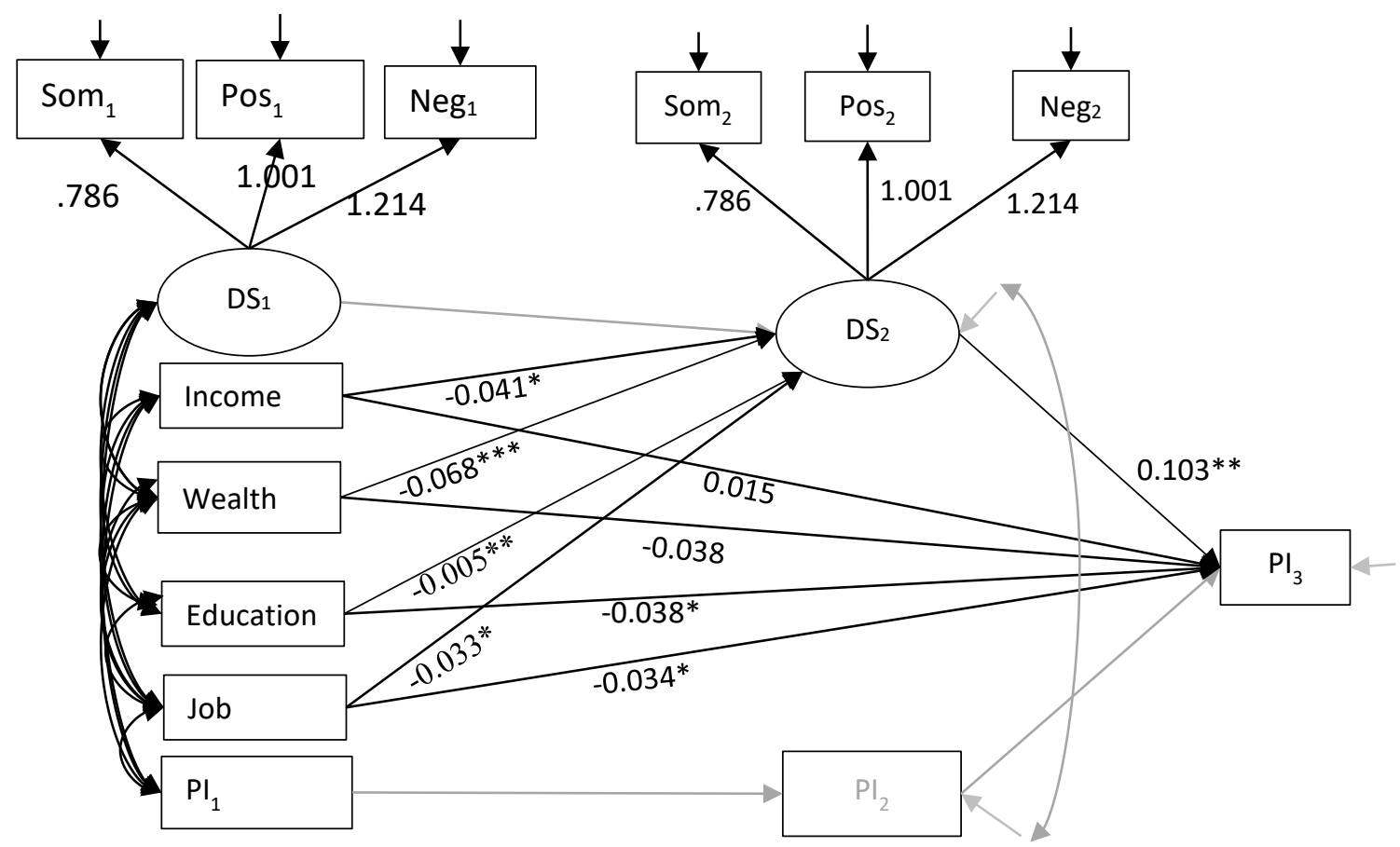

Figure 18: Lagged regression model for Whites $(\mathrm{N}=5717)$ with standardized path coefficients (Depressive Symptoms Mediator)

Note: $* \mathrm{p}<0.05 ; * * * \mathrm{p}<0.001$. Only paths pertinent to significant indirect relationships are shown. All control variables, paths modeled, variances and covariances are not shown to avoid clutter. $\mathbf{D S}=$ depressive symptoms, PI=physical impairment, $\mathbf{S o m}=$ somatic symptoms, $\mathbf{P o s}=$ positive affect (reverse coded), $\mathbf{N e g = n e g a t i v e ~ a f f e c t , ~ a n d ~ s u b s c r i p t s ~ i n d i c a t e ~ t i m e ~ p o i n t s . ~}$ 
Across race/ethnic groups, therefore, only education level appeared to be significantly related to depressive symptoms, suggesting the protective effects of higher education from depressive factors across groups.

The direction of the relationship between the mediating variable (depressive symptoms) and the outcome variable (physical impairment) was consistent with hypothesis 2a in all groups. Depressive symptoms scores were positively associated with physical impairment scores in the White group $\left(\beta=0.596, \mathrm{SE}=0.131, \beta^{*}=0.103, \mathrm{p}<0.001\right)$ and Hispanic group $\left(\beta=1.23, \mathrm{SE}=0.295, \beta^{*}=0.214, \mathrm{p}<0.001\right)$, adjusting for other $\mathrm{SES}$ and demographic variables. This finding lends support to the hypothesized relationship between depressive symptoms and physical impairment in these groups. In Blacks, depressive symptoms latent variable was not significantly related to physical impairment, however.

\subsubsection{Direct Effects of SES on Physical Impairment}

Higher SES was expected to be associated with lower physical impairment scores but these path coefficients need not be significant given the inclusion of the latent depressive symptoms variable as a mediator. The direct effects of educational level $\left(\beta_{\text {edu }}=-0.016, \mathrm{SE}=0.08, \beta_{\text {edu }}^{*}=-0.038, \mathrm{p}<0.05\right)$ and job status $\left(\beta_{\mathrm{job}}=-0.079, \mathrm{SE}=0.033\right.$, $\beta^{*}{ }_{\mathrm{job}}=-0.034, \mathrm{p}<0.05$ ), on physical impairment, adjusting for other demographic and SES variables were significant in the White group. On average, increase in educational level was associated with decrease in physical impairment scores and being employed was associated with lower physical impairment scores in Whites. 


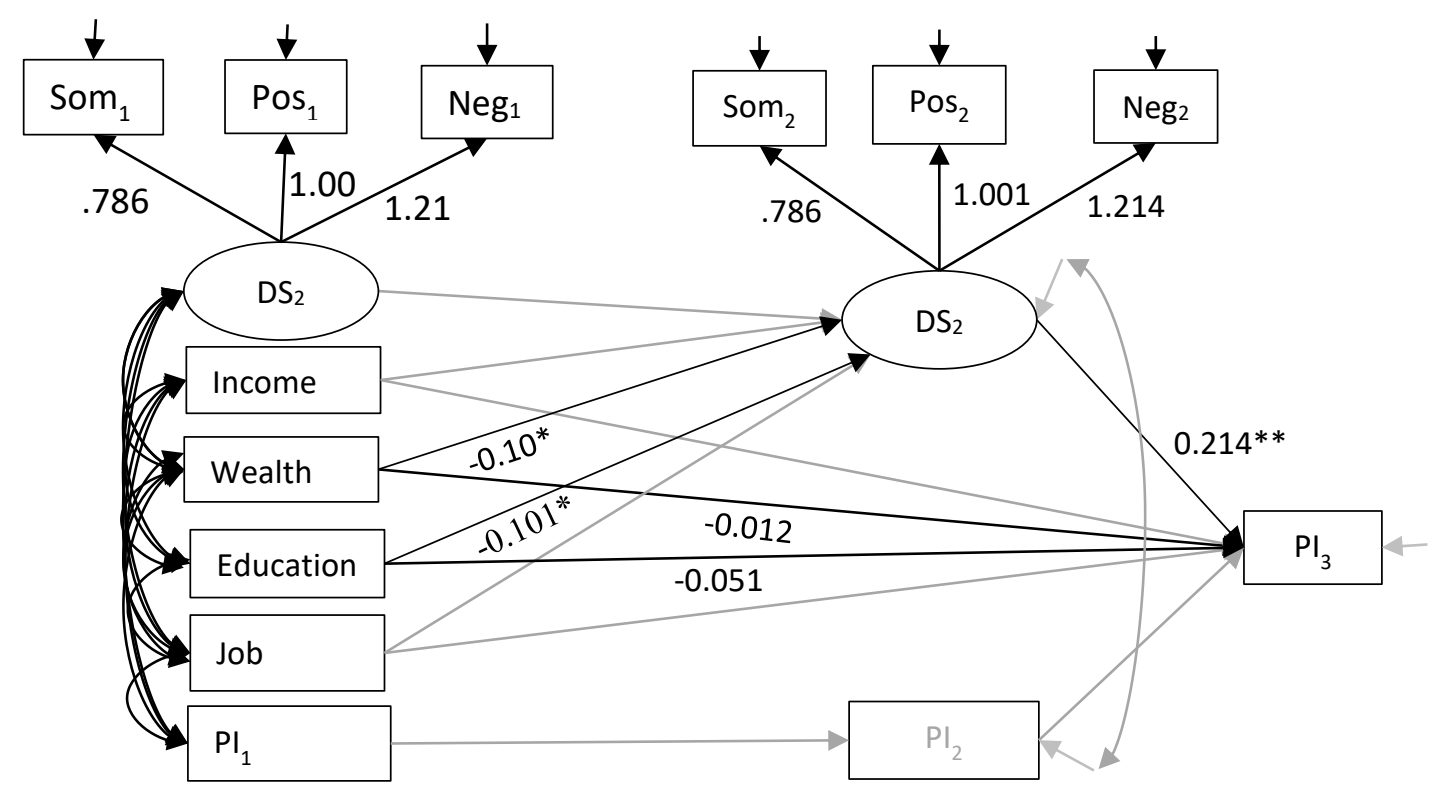

Figure 19: Lagged regression model for Hispanics $(\mathrm{N}=719)$ with standardized path coefficients (Depressive Symptoms Mediator)

Note: $* \mathrm{p}<0.05 ; * * * \mathrm{p}<0.001$. Only paths pertinent to significant indirect relationships are shown. All control variables, paths modeled, variances and covariances are not shown to avoid clutter. $\mathbf{D S}=$ depressive symptoms, $\mathbf{P I}=$ physical impairment, $\mathbf{S o m}=$ somatic symptoms, $\mathbf{P o s}=$ positive affect (reverse coded), $\mathbf{N e g}=$ negative affect, and subscripts indicate time points.

Only job status $\left(\beta_{\mathrm{job}}=-0.182, \mathrm{SE}=0.08, \beta^{*}{ }_{\mathrm{job}}=-0.056, \mathrm{p}<0.05\right)$ was significantly related to physical impairment status in Blacks, indicating that being employed was associated with decrease in physical impairment controlling for other demographic and SES variables. Similarly, in the Hispanic group, job status was significantly related to physical impairment status, $\left(\beta_{\mathrm{job}}=-0.412, \mathrm{SE}=0.101, \beta^{*}{ }_{\mathrm{job}}=-0.131, \mathrm{p}<0.001\right)$, suggesting that being employed was related to a large decrease in physical impairment scores. These results appeared to indicate larger protective effect of employment for Hispanics and Blacks. The next section examines indirect paths and differences in these paths by race/ethnicity. 


\subsubsection{Indirect Effects of SES on Physical Impairment}

Turning to the indirect relationship of each SES variable with physical impairment, the principal part of hypothesis $2 \mathrm{a}$ in this section, results showed varying degrees of indirect association between SES and physical impairment across race/ethnicity.

In the White group, income $\left(\beta_{\text {inc }}=-0.004, \mathrm{SE}=0.002, \beta^{*}{ }_{\text {inc }}=-0.004, \mathrm{p}<0.05\right)$, wealth $\left(\beta_{\text {wlth }}=-0.002, \mathrm{SE}=0.001, \beta^{*}{ }_{\text {wlth }}=-0.007, \mathrm{p}<0.05\right)$, education $\left(\beta_{\text {edu }}=-0.002, \mathrm{SE}=0.003, \beta^{*}=-\right.$ $0.005, \mathrm{p}<0.05)$ and job status $\left(\beta_{\mathrm{job}}=-0.008, \mathrm{SE}=0.001, \beta^{*}{ }_{\mathrm{job}}=-0.003, \mathrm{p}<0.05\right)$ were indirectly associated with physical impairment via depressive symptoms providing support to the hypothesized relationships in this section. Increase in income, wealth, education or being employed was associated with decrease in depressive symptoms at the second time point and decrease in depressive symptoms was, in turn, associated with decrease in physical impairment status at the third time point in the White group.

None of the SES variables were indirectly related to physical impairment in the Black group, indicating the indirect association of SES with physical impairment through depressive symptoms was not different from zero.

In the Hispanic group, only wealth $\left(\beta_{\mathrm{wlth}}=-0.005, \mathrm{SE}=0.002, \beta^{*}{ }_{\text {wlth }}=-0.021, \mathrm{p}\right.$ $<0.05)$ and education $\left(\beta_{\text {edu }}=-0.007, \mathrm{SE}=0.017, \beta^{*}\right.$ edu $\left.=-0.022, \mathrm{p}<0.05\right)$ were indirectly related to physical impairment, where, higher wealth and education scores were related to lower depressive symptoms and lower depressive symptoms, in turn, were related to lower physical impairment status. These indirect effect sizes were small. However, the 
overall variance accounted for in the psychological (depressive symptoms) variable was not trivial as indicated by large R-square for Whites ( $\mathrm{R}$-square=0.448, $\mathrm{SE}=0.030$, $\mathrm{p}<0.001)$, for Blacks (R-square $=0.505, \mathrm{SE}=0.065, \mathrm{p}<0.001)$ and for Hispanics $(\mathrm{R}-$ square $=0.425, \mathrm{SE}=0.061, \mathrm{p}<0.001)$. Variance accounted for in physical impairment across groups was also substantial as indicated in previous sections.

\subsubsection{Follow-up Analyses}

As a general follow-up test, I conducted nested chi-square analysis where direct and indirect paths from each SES variable to physical impairment were constrained equal across groups in one model and freely estimated in an alternative model. Results indicated that constraining all paths led to a significant worsening of fit $\left[\left(\Delta \chi^{2}(18)=29.88\right.\right.$, $\mathrm{p}<0.05)]$. These follow-up analyses results, therefore, provided evidence supporting differences in the indirect SES to physical impairment paths across race/ethnic groups.

Further invariance tests of specific paths were conducted to examine whether any of the indirect SES to physical health status paths were the same across groups. Constraining paths from income to physical impairment $\left[\left(\Delta \chi^{2}(6)=9.03\right.\right.$, ns $\left.)\right]$, or wealth to physical impairment $\left[\left(\Delta \chi^{2}(6)=7.19, \mathrm{~ns}\right)\right]$, or education to physical impairment $\left[\left(\Delta \chi^{2}(6)=7.72, \mathrm{~ns}\right)\right]$, or job status to physical impairment $\left[\left(\Delta \chi^{2}(6)=10.66, \mathrm{~ns}\right)\right]$, did not result in worsening of fit as indicated by non-significant $\Delta \chi^{2}$ for each nested model comparison. It, therefore, appears that the paths from SES to physical impairment were invariant across groups. 


\section{Summary}

In summary, the above results provided complete support to hypothesis $2 \mathrm{a}$ in the White group, where, increase in each SES variable was indirectly associated with decrease in physical impairment through a latent depressive symptoms variable. Partial support to the hypothesis was provided in the Hispanic group where only increases in wealth and education were indirectly associated with decrease in physical impairment overtime. The results, however, failed to provide support to hypothesis $2 \mathrm{a}$ in the Black group, where, none of the SES variables were significantly indirectly associated with physical impairment.

In the next section, a latent growth curve model was tested to examine trajectories of change across groups and investigate whether SES variables were indirectly related to physical impairment through depressive symptoms.

\subsection{SES-Psychological Factors-Physical Impairment: Growth Curve Model}

Hypothesis 2b: Each SES variable will be negatively related to the slope factor for depressive symptoms and the slope factor for physical impairment. The slope factor for depressive symptoms, in turn, will be positively related to the slope factor for physical impairment. These paths will differ across race/ethnic groups.

\subsubsection{Growth Curve Model for the Total Sample}

Latent growth curve mediation modeling approach affords several advantages over lagged path models as described earlier. This section examines whether SES is related to change in the slope factor for physical impairment status via change in the 
slope factor for depressive symptoms. Demographic variables were included as timeinvariant covariates.

In order to proceed with investigating the relationships between SES variables and the slope factor for depressive symptoms, and between the depressive symptoms slope factor and the physical impairment slope factor, I first specified a second order latent growth curve model for the total sample. Results showed that the model fit to the

data was acceptable, $\left[\left(\chi^{2}(268)=1594.85, \mathrm{CFI}=0.93, \mathrm{SRMR}=0.046\right)\right]$. Each SES variable was significantly related to the slope factor for physical impairment and the direction of the relationship was consistent with the hypothesis. Each SES to depressive symptoms slope factor path coefficient was not significant in the total sample, failing to support the hypothesized relationship, however. The standardized slope factor for depressive symptoms predicted the standardized slope factor for physical impairment ( $\beta=3.37$, $\left.\mathrm{SE}=0.920, \beta^{*}=0.415, \mathrm{p}<0.001\right)$, where, increase in the depressive symptoms growth rate was associated with increase in the slope factor for physical impairment every two years. None of the SES variables were indirectly related to the slope factor for physical impairment, however, failing to support the hypothesis in the total sample.

\subsubsection{Growth Curve Models for Individual Groups}

Although the latent growth curve model for the total sample above failed to support hypothesis $2 b$, it was not improbable for individual group models to differ and suggest variations across groups and provide support for the hypothesis. The next models provide further analysis of individual and multiple group models. The individual models 
for each race/ethnic group fit to the data well. Specifically, the model for White group $\left[\left(\chi^{2}(268)=1299.68, \mathrm{CFI}=0.95, \mathrm{SRMR}=0.050\right)\right]$, for the Black group, $\left[\left(\chi^{2}(268)=473.8\right.\right.$, $\mathrm{CFI}=0.96, \mathrm{SRMR}=0.048)]$, and for the Hispanic group, $\left[\left(\chi^{2}(268)=488.51, \mathrm{CFI}=0.94\right.\right.$, SRMR=0.050)], fit the data sufficiently well. After establishing a model that fits each group well, the next step examined direct and indirect SES to physical impairment paths in a multiple group modeling framework.

\subsubsection{Multiple Group Growth Curve Model}

This model addressed the relationship between the trajectory for depressive symptoms with the trajectory for physical impairment in a multiple group framework with time-invariant SES predictors and demographic covariates. The model fit to the data adequately, $\left[\left(\chi^{2}(815)=2712.58, \mathrm{CFI}=0.94, \mathrm{SRMR}=0.059\right)\right]$, supporting the plausibility of the specified model for the three groups.

The mean depressive symptoms for Whites $\left(\alpha_{\mathrm{w}}=-0.018, \mathrm{SE}=0.009 ; \alpha_{\mathrm{w}}{ }^{*}=-0.827\right.$, $\mathrm{p}<0.05)$ declined over time and this decrease varied among the White respondents. In the Black group, mean depressive symptoms declined over time $\left(\alpha_{\mathrm{b}}=-0.031, \mathrm{SE}=0.015 ; \alpha_{\mathrm{b}}{ }^{\prime}=-\right.$ $1.268, \mathrm{p}<0.05)$ but did not vary between Black respondents. Depressive symptoms in the Hispanic group also tended to decline $\left(\alpha_{\mathrm{h}}=-0.016, \mathrm{SE}=0.024 ; \alpha_{\mathrm{h}}^{*}=-0.595, \mathrm{~ns}\right)$ over time but this tendency was not significant.

As would be expected for an older sample, the slope for physical impairment was significant in each group, indicating physical impairment in each group increased over 
time. In Whites, the physical impairment slope factor $\left(\alpha_{\mathrm{w}}=0.235, \mathrm{SE}=0.062 ; \alpha^{*}{ }_{\mathrm{w}}=1.337\right.$, $\mathrm{p}<0.001$ ), significantly increased over time and this growth varied between individuals. Similarly, in Blacks physical impairment scores increased over time $\left(\alpha_{\mathrm{b}}=0.476\right.$, $\left.\mathrm{SE}=0.158 ; \alpha^{*}=1.874, \mathrm{p}<0.05\right)$ but increase did not vary within the group. In Hispanics, increase in physical impairment scores $\left(\alpha_{\mathrm{h}}=0.390, \mathrm{SE}=0.192 ; \alpha_{\mathrm{h}}{ }_{\mathrm{h}}=1.752, \mathrm{p}<0.05\right)$ was also significant but did not vary within the group.

In the next sections, I examine the direct and indirect paths from SES to the slope growth factors of physical impairment and depressive symptoms.

\subsubsection{Direct Effects of SES on the Growth Factor of Psychological Status}

I hypothesized that each of the SES variables would be negatively related to the slope factor for depressive symptoms. In the Hispanic and White groups, none of the paths from SES to the slope factor for depressive symptoms were significant failing to support the hypothesized relationship between SES and the slope factor for depressive symptoms.

For Blacks, the direct effect of income on the slope factor for depressive symptoms was marginally significant, $\left(\beta_{\mathrm{inc}}=0.004, \mathrm{SE}=0.002, \beta^{*}{ }_{\mathrm{inc}}=0.218, \mathrm{p}<0.05\right)$, but in the opposite direction than hypothesized, where, higher income was associated with higher scores of depressive symptoms. Income in this case was an inconsistent mediator because the sign of the product of the indirect coefficients was opposite of the sign of the coefficient for the direct effect. All other SES variables were not significantly related to depressive symptoms in the Black group. 


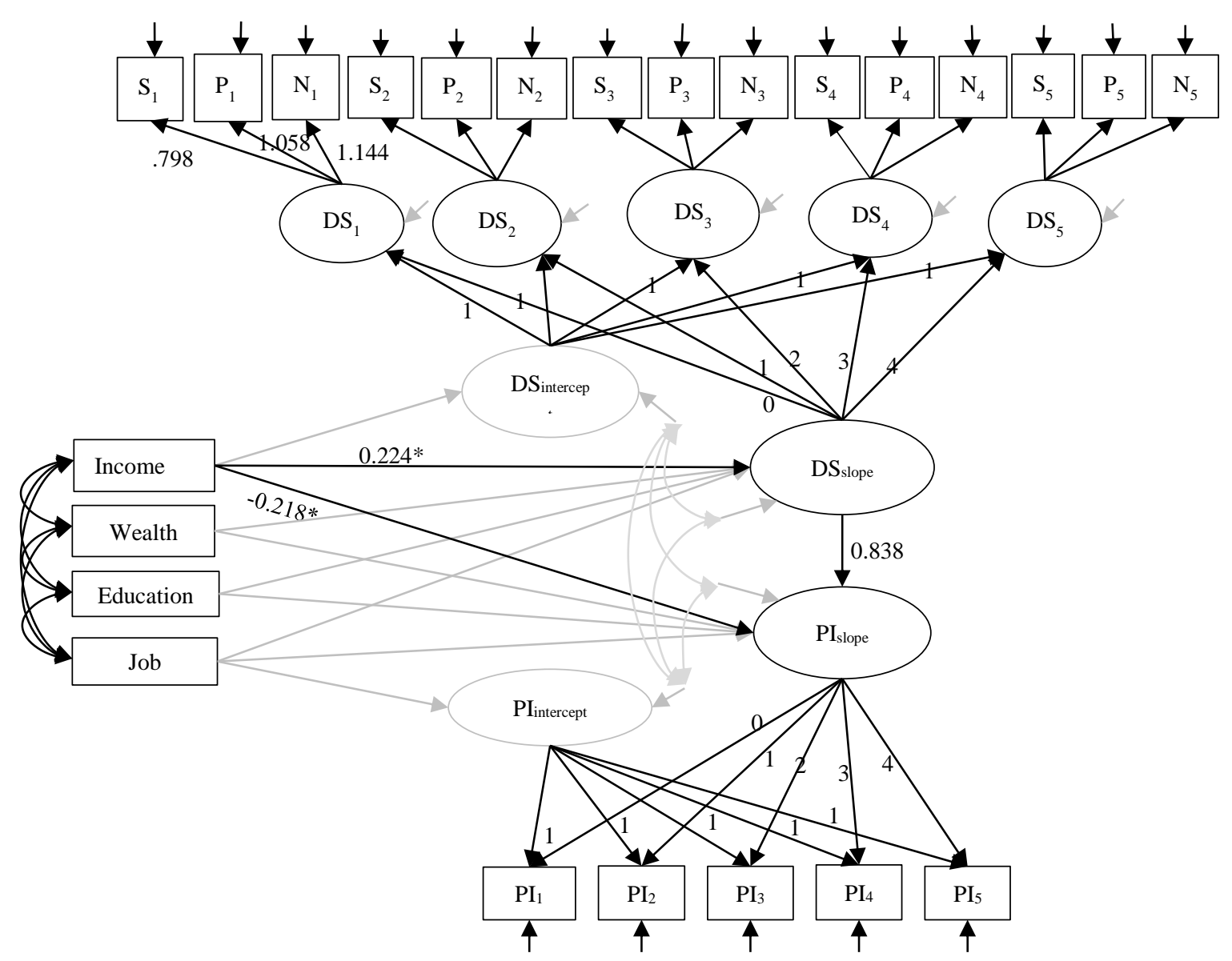

Figure 20: Second order latent growth curve model for Blacks (N=1238) with standardized path coefficients (Depressive Symptoms Mediator)

Note: $* p<0.05 ; * * * p<0.001$, subscripts indicate time points. Only paths pertinent to significant indirect relationships are shown. All control variables, paths modeled, variances, covariances, and correlated errors are not shown to avoid clutter. DS=depressive symptoms, $\mathbf{P I = p h y s i c a l ~ i m p a i r m e n t , ~} S=$ somatic symptoms, $P=$ positive affect (reverse coded) \& $N=$ negative affect. Second level factor loadings are constrained equal across time and across groups; residual errors for the second order indicators are correlated across time.

Loadings are held invariant over time and across groups.

For the depressive symptoms slope factor to physical impairment slope factor relationships, results indicated that the depressive symptoms slope factor was significantly related to the physical impairment slope factor in the White group, $(\beta=2.45$, $\left.\mathrm{SE}=0.95, \beta^{*}=0.297, \mathrm{p}<0.05\right)$. This was consistent with the hypothesized relationship that the trajectory of depressive symptoms would be positively related to the trajectory of 
physical impairment scores, in which, increase in the slope factor for depressive symptoms was associated with increase in the slope factor for physical impairment in the White group.

In the Black group, the depressive symptoms slope factor was only marginally related to the physical impairment slope factor, $\left(\beta=8.67, \mathrm{SE}=4.61 \beta^{*}=0.84, \mathrm{p}=0.056\right)$, whereas in the Hispanic group, this relationship was not significantly different from zero $\left(\beta=5.63, \mathrm{SE}=4.99, \beta^{*}=0.763, \mathrm{~ns}\right)$. On average, increase in the slope factor for depressive symptoms was related to a large increase in the slope factor for physical impairment in the Black group. These path results for the White and Black groups provide support to hypothesis $2 b$.

\subsubsection{Direct Effects of SES on the Growth Factor for Physical Impairment}

Higher SES was expected to be associated with lower physical impairment scores over time, where, each SES variable would be negatively related the physical impairment slope factor. In Whites, wealth was significantly related to the slope factor for physical impairment, $\left(\beta_{\mathrm{wlth}}=-0.007, \mathrm{SE}=0.002, \beta^{*}{ }_{\text {wlth }}=-0.144, \mathrm{p}<0.05\right)$, indicating that wealthier Whites, on average, had lower physical impairment scores over time. The direct effects of education $\left(\beta_{\text {edu }}=-0.002, \mathrm{SE}=0.002, \beta_{\text {edu }}^{*}=-0.035, \mathrm{p}<0.05\right)$ and employment $\left(\beta_{\mathrm{job}}=-0.031\right.$, $\left.\mathrm{SE}=0.009, \beta^{*}{ }_{\text {job }}=-0.086, \mathrm{p}<0.05\right)$ on physical impairment slope factor in the White group were also significant. Thus, in the White group, on average, increase in education was associated with decrease in the slope factor for physical impairment and being employed was associated with decrease in the slope factor for physical impairment, controlling for 
other SES and demographic variables. In the Black group, only income was significantly related to physical impairment slope factor, $\left(\beta_{\text {inc }}=-0.038, \mathrm{SE}=0.016, \beta^{*}{ }_{\text {inc }}=-0.224, \mathrm{p}\right.$ $<0.05)$, where, increase in income for Blacks was associated with decrease in the slope factor for physical impairment, adjusted for other SES and demographic variables. None of the other SES variables were significantly related to physical impairment slope factor, controlling for other SES and demographic variables in Blacks. Likewise, none of the SES variables were significantly related to the slope factor for physical impairment in Hispanics.

\subsubsection{Indirect Effects of SES on Physical Impairment}

The objective in this multiple group growth curve model was to examine whether each SES was related to the slope factor for depressive symptoms and whether the slope factor for depressive symptoms was related to the slope factor for physical impairment to quantify the indirect association between each SES variables and physical impairment. Examining the indirect effects for each SES variable in each race/ethnic group indicated that only in the Black group and only the indirect effect of income on physical impairment via the depressive symptoms slope factor was marginally significant, $\left(\beta_{\mathrm{inc}}=0.031, \mathrm{SE}=0.016, \beta_{\mathrm{inc}}^{*}=0.183, \mathrm{p}=0.054\right)$, but the relationship was in the opposite direction than hypothesized (inconsistent mediation). In the Black group, the relationship between income and depressive symptoms slope factor was positive, whereas, hypothesis 2a proposed this relationship would be negative. This was an unexpected result and it suggests a suppression effect in the model for Blacks. All other indirect paths were not significantly different from zero, failing to provide evidence in support of the postulated 
relations that each SES variable was related to the slope factor for physical impairment through the slope factor for depressive symptoms in all groups.

\section{Summary}

To summarize, the underlying hypothesis that SES indirectly predicts the trajectory of physical impairment via the trajectory of depressive symptoms was not supported in latent growth curve mediation modeling framework. Note that mean change (growth) in depressive symptoms scores over time did not appear substantial as indicated in Figure 12. Recall that in the path model above (hypothesis $2 a$ ) the time between adjacent data collection points was longer than that for the growth curve model. The path model also controlled for all dependent variables at a previous time point which could be the reason for significant paths in the lagged model but not in the growth curve model.

\subsection{SES-Behavioral Factors-Physical Impairment: Lagged Path Model}

Hypothesis 3: Each SES variable will be negatively related to body mass index [BMI], alcoholic beverage consumption and physical impairment. Body mass index and alcoholic beverage consumption, in turn, will be positively related to physical impairment scores. These paths will differ across racelethnic groups.

\section{Lagged Regression Path Model}

The objective of this section was to examine how SES variables were indirectly related to physical impairment via behavioral factors. Body mass index was used as one of the behavioral variable where higher SES individuals were presumed to have access to healthful foods, facilities for physical activity, health information and possibly act to slow 
physical impairment. Similarly, higher SES individuals were expected to have lower levels of alcoholic beverage consumption, which, in turn, was expected to be related to lower physical impairment. The next sections report fit results for total and group models, as well as direct and indirect path coefficients.

\subsubsection{Path Model for the Total Sample}

A lagged regression model with two behavioral factors as mediators was fit to the total sample to examine overall fit of the model to the data. Results suggested that the model fit to the data well, $\left[\left(\chi^{2}(5)=169.55, \mathrm{CFI}=0.98, \mathrm{SRMR}=0.014\right)\right]$. The only SES variable that had significant association with BMI was wealth $\left(\beta_{\mathrm{wlth}}=-0.028, \mathrm{SE}=0.011\right.$, $\left.\beta^{*}{ }_{\text {wlth }}=-0.022, \mathrm{p}<0.05\right)$, where, in the total sample, increase in wealth was associated with decrease in BMI. For the second mediator, namely alcoholic beverage consumption, employment status $\left(\beta_{\mathrm{job}}=-0.039, \mathrm{SE}=0.019 \beta_{\mathrm{job}}^{*}=-0.021, \mathrm{p}<0.05\right)$ and education $\left(\beta_{\text {edu }}=0.01, \mathrm{SE}=0.003, \beta^{*}{ }_{\text {edu }}=0.033, \mathrm{p}<0.001\right)$ were significantly related to alcoholic beverage consumption. Being employed was related to a small decrease in alcoholic consumption consistent with the hypothesized relationships. However, contrary to hypothesis 3 , on average, higher level of education was related to higher level of alcoholic beverage consumption. Wealth was marginally related to alcoholic beverage consumption $\left(\beta_{\mathrm{wlth}}=0.003, \mathrm{SE}=0.001, \beta^{*}{ }_{\text {wlth }}=0.013, \mathrm{p}=0.054\right)$ but in the opposite direction than that proposed in hypothesis 3 . Wealthier people, on average, appeared to drink more alcoholic beverages. 
Regarding the direct effects, each SES variable was expected to be negatively related to physical impairment. Wealth $\left(\beta_{\mathrm{wlth}}=-0.016, \mathrm{SE}=0.004, \beta^{*}{ }_{\text {wlth }}=-0.057, \mathrm{p}<0.001\right)$, employment $\left(\beta_{\mathrm{job}}=-0.121, \mathrm{SE}=0.031, \beta^{*}\right.$ job $\left.=-0.049, \mathrm{p}<0.001\right)$ and education $\left(\beta_{\text {edu }}=-0.018\right.$, $\left.\mathrm{SE}=0.007, \beta_{\text {edu }}^{*}=-0.045, \mathrm{p}<0.05\right)$, were negatively associated with physical impairment as hypothesized.

Consistent with hypothesis 3 , the path coefficient from BMI to physical impairment $\left(\beta=0.011, \mathrm{SE}=0.004, \beta^{*}=0.051, \mathrm{p}<0.05\right)$ was significant, but the path coefficient from alcoholic beverage consumption to physical impairment was not significant. Given the various direct and indirect path coefficients, I examined the mediating role of BMI and alcoholic beverage consumption. As suspected, due to small path coefficients, none of the specific indirect paths from SES to physical impairment via BMI or alcoholic beverage consumption were significant, failing to support hypothesis 3 in the total sample.

In the next section, a multiple group lagged path model was tested to examine indirect path coefficients for each group.

\subsubsection{Multiple Group Path Model}

As provided above, in the total sample, indirect relationships between SES and physical impairment failed to support hypothesis 3. It is possible, however, that paths may indicate significant relationships in individual groups. With that in mind, I examined a multiple group lagged path model. The multiple group lagged path model fit to the data well, $\left[\left(\chi^{2}(23)=212.76, \mathrm{CFI}=0.98, \mathrm{SRMR}=0.015\right)\right]$, suggesting that the model was 
reasonable for investigating the hypothesized relationships in a multiple group framework. Given the fit of this model, the next sections examine multi-group lagged path models to test indirect associations between SES and physical impairment through BMI and alcoholic beverage consumption.

\subsubsection{Direct Effects of SES on Body Mass Index (BMI) and Alcohol \\ Consumption}

Across race/ethnic groups, the SES to BMI and SES to alcoholic beverage consumption path coefficients were mostly non-significant with a few exceptions. In the White group, wealth $\left(\beta_{\mathrm{wlth}}=-0.041, \mathrm{SE}=0.015, \beta^{*}{ }_{\text {wlth }}=-0.029, \mathrm{p}<0.05\right)$ was significantly associated with BMI such that increase in wealth was associated with a small decrease in BMI. However, none of the other SES variables were significantly related to BMI or alcoholic beverage consumption in the White group. Similarly, none of the SES variables were significantly related to BMI or alcoholic beverage consumption in the Hispanic or Black groups, failing to support the hypothesized relationship between SES and the proposed mediators in these groups.

\subsubsection{Direct Effects of SES on Physical Impairment}

Overall, across all race/ethnic groups, each SES was negatively related to physical impairment status as hypothesized. In the White group, wealth $\left(\beta_{\text {wlth }}=-0.014, \mathrm{SE}=0.007\right.$, $\left.\beta_{\text {wlth }}^{*}=-0.049, \mathrm{p}=0.051\right)$, employment status $\left(\beta_{\mathrm{job}}=-0.095, \mathrm{SE}=0.032, \beta^{*}{ }_{\mathrm{job}}=-0.041, \mathrm{p}\right.$ $<0.05)$ and education level $\left(\beta_{\text {edu }}=-0.022, \mathrm{SE}=0.008, \beta^{*}\right.$ edu $\left.=-0.051, \mathrm{p}<0.05\right)$ were each significantly related to physical impairment scores controlling for other SES and 
demographic variables. Similarly, in Blacks, the direct effect of wealth $\left(\beta_{\text {wlth }}=-0.018\right.$, $\left.\mathrm{SE}=0.008, \beta^{*}{ }_{\text {wlth }}=-0.072, \mathrm{p}<0.05\right)$ and employment status $\left(\beta_{\mathrm{job}}=-0.212, \mathrm{SE}=0.083, \beta_{\mathrm{job}}^{*}=-\right.$ $0.066, \mathrm{p}<0.05$ ) on physical impairment were significant. As in the White and Black groups, employment status $\left(\beta_{\mathrm{job}}=-0.427, \mathrm{SE}=0.105, \beta_{\mathrm{job}}^{*}=-0.136, \mathrm{p}<0.001\right)$ was significantly related to physical impairment status, whereas, wealth $\left(\beta_{\text {wth }}=-0.011\right.$, $\left.\mathrm{SE}=0.006, \beta^{*}{ }_{\text {wlth }}=-0.043, \mathrm{p}=0.07\right)$ was marginally significantly related to physical impairment in the Hispanic group, controlling for other SES/demographic variables.

\subsubsection{Indirect Effects of SES on Physical Impairment}

The adjusted path coefficients presented above were quite small signaling that the indirect effects might also be weak or non-significant at all because the indirect effects are products of these path coefficients. As suspected, only wealth was significantly indirectly associated with physical impairment via BMI, $\left(\beta_{\mathrm{wlth}}=-0.001, \mathrm{SE}=0.00, \beta^{*}{ }_{\text {wlth }}=-\right.$ $0.001, \mathrm{p}<0.05)$ in the White group, where, increase in wealth was indirectly related to a small decrease in physical impairment in the White group. All other indirect SES to physical impairment paths in the White group were not significant failing to provide evidence supporting the hypothesis.

In the Hispanic and Black groups, none of the SES variables were indirectly related to physical impairment via BMI and alcoholic beverage consumption. This suggested that, in this study, the hypothesized mediating role of alcoholic beverage consumption and BMI in the SES-physical impairment link was not supported. Furthermore, results indicated that the direction of hypothesized relationship between 
SES and alcoholic beverage consumption was not supported because higher education and wealth, for instance, were associated with higher alcohol consumption in this data. The overall variance accounted for in BMI was substantial across groups. Variance accounted for in BMI in the White group (R-square=0.801, $\mathrm{SE}=0.012, \mathrm{p}<0.001)$, in the Black group $(\mathrm{R}$-square $=0.759, \mathrm{SE}=0.032, \mathrm{p}<0.001)$ and the Hispanic group $(\mathrm{R}-$ square $=0.691, \mathrm{SE}=0.041, \mathrm{p}<0.001)$ were significant. Similarly, variance accounted for in the alcoholic beverage consumption variable in Whites $(\mathrm{R}$-square $=0.550, \mathrm{SE}=0.038$, $\mathrm{p}<0.001)$, in Blacks $(\mathrm{R}$-square $=0.362, \mathrm{SE}=0.116, \mathrm{p}<0.05)$ and in Hispanics $(\mathrm{R}$ square $=0.338, \mathrm{SE}=0.079, \mathrm{p}<0.001)$ were not trivial, but smaller in the model for the Black and Hispanic groups.

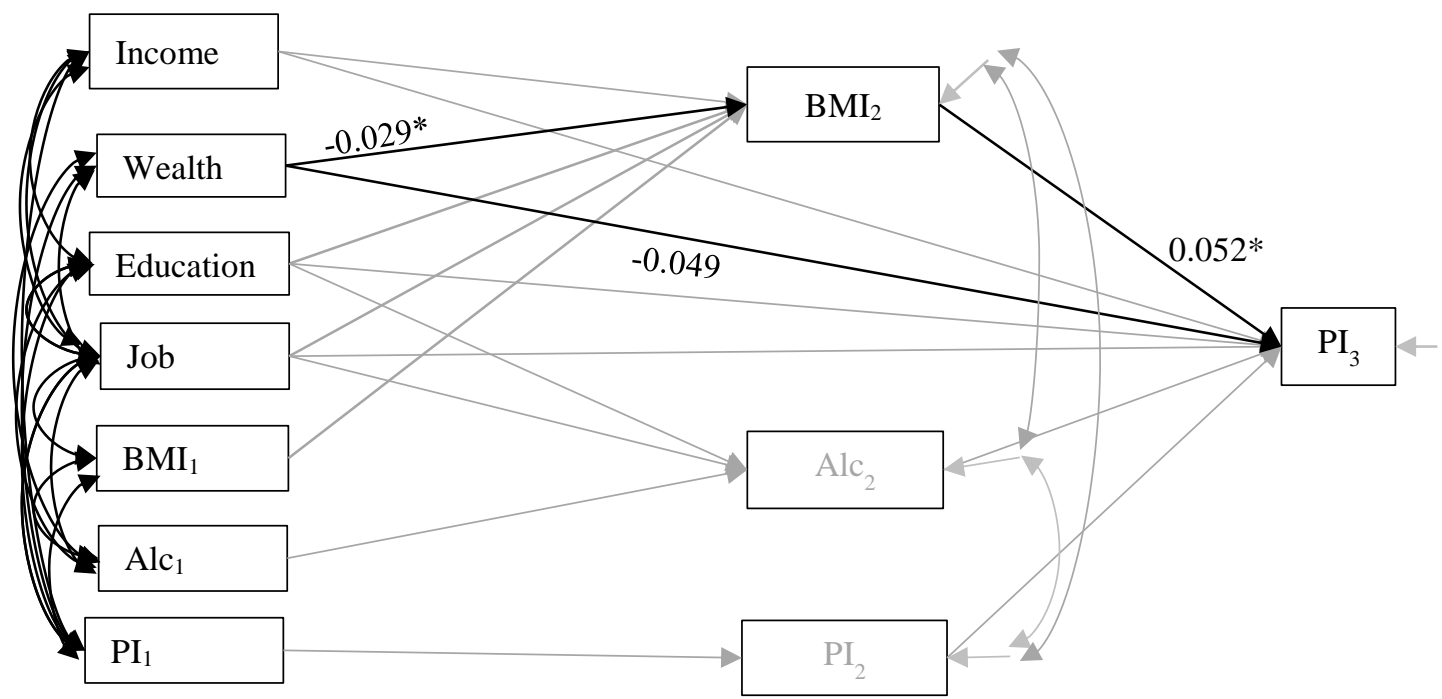

Figure 21: Lagged regression model for Whites $(\mathrm{N}=5717)$ with standardized path coefficients (Behavioral variable Mediators)

Note: $*$ p $<0.05$. Only paths pertinent to significant indirect relationships are shown. All control variables, paths modeled, variances and covariances are not shown to avoid clutter. Alc=alcoholic beverage consumption, BMI=body mass index, $\mathbf{P I}=$ physical impairment. Subscripts indicate time points. 


\section{Follow-up Analysis}

To examine invariance of indirect path coefficients across groups for the effect of wealth on physical impairment through body mass index, I tested chi-square difference between constrained and freely estimated models. Constraining wealth to BMI and wealth to physical impairment as well as BMI to physical impairment paths did not significantly worsen fit $\left[\left(\Delta \chi^{2}(6)=11.26, \mathrm{~ns}\right)\right]$ suggesting paths were not different across groups.

\section{Summary}

In summary, results failed to provide support for hypothesis 3 , showing that the SES to BMI, and BMI to physical impairment path coefficients were not significantly different from zero except for the indirect association of wealth with physical impairment in the White group. Invariance tests for this path indicated that the effects were not significantly different across groups, however. Socioeconomic status to alcoholic beverage consumption, and alcoholic beverage consumption to physical impairment path coefficients were not significantly different from zero across groups. The overall direction of association between SES variables and alcoholic beverage consumption variable was not consistent with hypothesis 3, where respondents in higher SES positions, on average, consumed higher levels of alcoholic beverage.

\section{Overnight Hospitalization}

The second outcome variable in this study was overnight hospitalization - a binary variable $(1=$ yes and $0=$ no $)$ - indicating whether a respondent had any overnight 
hospital stay during the previous two years. Because the outcome variable in the following hypotheses (4-6 below) was binary, regular ordinary least squares regression (OLS) approach was not appropriate due to violation of linear regression assumptions. One way that this is addressed is by assuming that the binary variable measures an underlying continuous variable and using threshold levels for the outcome variable as described in the methods section (Chapter 4), which was used in the analyses that follow. Further explanation of this rationale can be found in (MacKinnon, 2008).

\subsection{SES-Social Factors-Overnight Hospitalization: Lagged Path Model}

Hypothesis 4: Each SES variable will be negatively related to overnight hospitalization but positively related to social integration. Social integration, in turn, will be negatively related to overnight hospitalization. These paths will differ across racelethnic groups.

\section{Lagged Regression Path Model for the Social Integration Mediating Variable}

A lagged regression model was fit to examine the indirect relationship of each SES variable to overnight hospitalization via social integration. Each SES variable was expected to be positively related to social integration. Actions that enhanced positive interactions with others, that is, high levels of social integration, were expected to reduce incidences of illness that may be associated with hospitalization. To test hypothesis 4 , this model first examined the relationship between SES and social integration, and the relationship between social integration and overnight hospitalization, specifically the indirect relationship between SES and overnight hospitalization for the total sample and then for multiple groups. Results are presented in the next sections. 


\subsubsection{Path Model for the Total Sample}

The rationale for the total sample path model was to examine whether SES was indirectly associated with overnight hospitalization in the total sample. The path model for the total sample fit to the data well, $\left[\left(\chi^{2}(3)=7.09, \mathrm{CFI}=0.99, \mathrm{RMSEA}=0.013\right)\right]$, suggesting that, the model was plausible.

As hypothesized, in the total sample, SES was inversely related to overnight hospitalization. Each SES variable, except employment status, was positively related to social integration at the second time point. Income $\left(\beta_{\mathrm{inc}}=0.045, \mathrm{SE}=0.009, \beta^{*}{ }_{\mathrm{inc}}=0.047, \mathrm{p}\right.$ $<0.001)$, wealth $\left(\beta_{\mathrm{wlth}}=0.015, \mathrm{SE}=0.003, \beta^{*}{ }_{\text {wlth }}=0.053, \mathrm{p}<0.001\right)$, and education $\left(\beta_{\text {edu }}=0.014, \mathrm{SE}=0.005, \beta^{*}{ }_{\text {edu }}=0.034, \mathrm{p}<0.05\right)$ were significantly related to social integration. Social integration to overnight hospitalization path was not significant, however, failing to support the hypothesized relationship. Although, the social integration to overnight hospitalization path coefficient was not significant in the total sample, it was essential to examine whether this was also true for each race/ethnic group. Thus, the next section considers multiple group lagged path models to examine indirect associations between SES and overnight hospitalization through social integration.

\subsubsection{Multiple Group Path Model}

Although there was not significant indirect relationship between each SES and overnight hospitalization through social integration, the path model was extended to a multiple group model to examine whether any group specific indirect paths were present.

The multiple group model fit to the data well, $\left[\left(\chi^{2}(9)=17.02, p=0.049, \mathrm{CFI}=0.99\right.\right.$, 
RMSEA=0.019)]. Direct and indirect path coefficients are explored for each group below.

\subsubsection{Direct Effects of SES on Social Integration}

In the White group, income $\left(\beta_{\text {inc }}=0.052, \mathrm{SE}=0.013, \beta^{*}{ }_{\text {inc }}=0.048, \mathrm{p}<0.001\right)$ and wealth $\left(\beta_{\text {wlth }}=0.011, \mathrm{SE}=0.005, \beta^{*}{ }_{\text {wlth }}=0.035, \mathrm{p}<0.05\right)$ were significantly related to social integration such that higher income and wealth were related to higher social integration scores. Increase in income was associated with increase in social integration and increase in wealth was associated with increase in social integration, controlling for other SES variables and covariates in the model. Education level was marginally related to social integration in the White group, $\left(\beta_{\text {edu }}=0.012, \mathrm{SE}=0.006, \beta^{*}\right.$ edu $\left.=0.026, \mathrm{p}=0.057\right)$, such that increase in education was associated with increase in social integration. In the Black group, only wealth $\left(\beta_{\mathrm{wlth}}=0.018, \mathrm{SE}=0.005, \beta^{*}{ }_{\text {wlth }}=0.084, \mathrm{p}<0.001\right)$, was significantly related to social integration, while income $\left(\beta_{\mathrm{inc}}=0.048, \mathrm{SE}=0.027, \beta^{*}{ }_{\mathrm{inc}}=0.057, \mathrm{p}=0.08\right)$ was marginally related to social integration. In Blacks, increase in wealth was associated with small increase in social integration scores. Only wealth $\left(\beta_{\mathrm{wth}}=0.017, \mathrm{SE}=0.008\right.$, $\beta^{*}{ }_{\text {wlth }}=0.08, \mathrm{p}<0.05$ ) was significantly associated with small social integration in the Hispanic group, where, increase in wealth was related to increase in social integration. Wealth had similar relationship to social integration in all groups, indicating increase in wealth was related to a small increase in social integration but the magnitude of change in social integration due to increase in wealth appeared to be higher for Blacks and Hispanics. 
In each group, the social integration to overnight hospitalization path coefficient was not significant, failing to provide evidence in favor of hypothesis 4 . Higher social integration scores seemed ineffectual on rates of overnight hospitalization, controlling for SES and demographic variables in all race/ethnic groups.

\subsubsection{Direct Effects of SES on Overnight Hospitalization}

Socioeconomic status variables were expected to be negatively associated with overnight hospitalization. In the White and Black groups, none of the SES variables were significantly related to overnight hospitalization, but in the Hispanic group, income $\left(\beta_{\text {inc }}=-0.141, \mathrm{SE}=0.06, \beta_{\text {inc }}^{*}=-0.205, \mathrm{p}<0.05\right)$ was significantly related to overnight hospitalization while education $\left(\beta_{\text {edu }}=-0.031, \mathrm{SE}=0.018, \beta^{*}{ }_{\text {edu }}=-0.111, \mathrm{p}=0.082\right)$ was marginally related to overnight hospitalization such that increases in income and education were associated with decrease in overnight hospitalization.

\subsubsection{Indirect Effects of SES on Overnight Hospitalization via Social Integration}

The overarching objective of the model was to examine indirect relationships between SES and overnight hospitalization via social integration and whether these relationships differed across race/ethnic groups. Results showed that none of the indirect paths from each SES to overnight hospitalization, via social integration, were significant. This was obvious given the results above showed that the social integration to overnight hospitalization path coefficients were not significantly different from zero in each race/ethnic group. 


\section{Summary}

In summary, results in this section failed to support hypothesis 4. Although higher SES was associated with higher social integration, higher social integration was not associated with overnight hospitalization in this study. Consequently, none of the SES variables were indirectly related to overnight hospitalization in any race/ethnic groups.

\subsection{SES-Psychological Factors-Overnight Hospitalization: Lagged Path Model}

Hypothesis 5: Each SES variable will be negatively related to overnight hospitalization and depressive symptoms. Depressive symptoms, in turn, will be positively related to overnight hospitalization. These paths will differ across racelethnic groups.

\section{Lagged Regression Path Model for Depressive Symptoms Mediating Variable}

Hospitalization as one of the forms of healthcare services use may indicate either resource use by people with higher SES or it may indicate poor health due to one's lower SES. Hypothesis 5 sought to examine the protective effects of SES from overnight hospitalization by reducing depressive symptoms. These indirect relationships may also differ across race/ethnic groups. Lagged regression modeling approach was used to examine whether SES was indirectly related to overnight hospitalization via depressive symptoms and whether these paths differed across race/ethnic groups. Note that the depressive symptoms variable (psychological variable) was a latent variable.

\subsubsection{Path Model for the Total Sample}

To investigate whether SES was related to depressive symptoms at the second time point and whether depressive symptoms were, in turn, related to overnight 
hospitalization at the third time point, I fit a lagged regression model to the total sample.

Results indicated that the proposed model fit to the data well, $\left[\left(\chi^{2}(47)=360.63, \mathrm{CFI}=0.96\right.\right.$, RMSEA $=0.029]$.

Socioeconomic statuses to depressive symptoms path coefficients were significant with the exception of the path coefficient from employment status to depressive symptoms. Income $\left(\beta_{\text {inc }}=-0.004, \mathrm{SE}=0.001, \beta_{\text {inc }}^{*}=-0.035, \mathrm{p}<0.05\right)$ and wealth $\left(\beta_{\mathrm{wlth}}=-\right.$ $\left.0.003, \mathrm{SE}=0.000, \beta^{*}{ }_{\text {wlth }}=-0.088, \mathrm{p}<0.001\right)$, were significantly related to depressive symptoms where increases in income and wealth were related to decrease in depressive symptoms. Education $\left(\beta_{\text {edu }}=-0.141, \mathrm{SE}=0.06, \beta_{\text {edu }}^{*}=-0.205, \mathrm{p}=0.073\right)$, was marginally significantly related to depressive symptoms. Considering the direct effects of SES on overnight hospitalization, only employment status $\left(\beta_{\text {edu }}=-0.115, \mathrm{SE}=0.058, \beta_{\text {edu }}^{*}=-0.048\right.$, $\mathrm{p}<0.05)$ was significantly related to overnight hospitalization indicating that those employed were less likely to experience overnight hospitalization. Further, the depressive symptoms variable to overnight hospitalization path coefficient in the total sample was not significant. The primary aim of this model was to examine the indirect relationships between each SES and overnight hospitalization by race/ethnicity. In the total sample, results showed that none of the SES variables were indirectly related to overnight hospitalization through depressive symptoms. Although these results point to possible lack of indirect associations in the data, further analysis of these paths by race/ethnicity was conducted to establish if the above results also held in each group. 


\subsubsection{Multiple Group Path Model}

The above analyses showed that indirect path coefficients were not significantly different from zero in the total sample. The outcome may be different for race/ethnic groups, however. Therefore, multiple group analysis was conducted to examine if there were any significant indirect path coefficients and whether these paths differed by race/ethnicity. The multiple group path model results indicated that the model had adequate fit to the data, $\left[\left(\chi^{2}(153)=500.2, \mathrm{CFI}=0.95, \mathrm{RMSEA}=0.030\right]\right.$. Adjusted direct and indirect SES to overnight hospitalization path coefficients are examined below.

\subsubsection{Direct Effects of SES on Depressive Symptoms}

Results for the White group were similar to the total sample results given above. Income $\left(\beta_{\text {inc }}=-0.004, \mathrm{SE}=0.001, \beta^{*}{ }_{\text {inc }}=-0.03, \mathrm{p}<0.05\right)$ and wealth $\left(\beta_{\mathrm{wlth}}=-0.04, \mathrm{SE}=0.000\right.$, $\left.\beta^{*}{ }_{\text {wlth }}=-0.099, \mathrm{p}<0.001\right)$ were significantly related to depressive symptoms. Increases in income and wealth were associated with decrease in depressive symptoms. Employment status and education level were not significantly related to depressive symptoms in the White group, however. In the Hispanic group, only wealth $\left(\beta_{\text {wlth }}=-0.004, \mathrm{SE}=0.002\right.$, $\left.\beta^{*}{ }_{\text {wlth }}=-0.115, \mathrm{p}<0.05\right)$ was significantly related to depressive symptoms, where, increase in wealth was related to a small decrease in depressive symptoms consistent with the hypothesis in this section. None of the other SES to depressive symptoms path coefficients was significant in the Hispanic group. Similarly, in the Black group, each path coefficient from each SES variable to depressive symptoms was not significantly different from zero. 
The path coefficients from depressive symptoms to overnight hospitalization were not significantly different from zero across groups failing to support the hypothesized relationships between depressive symptoms and overnight hospitalization.

\subsubsection{Direct Effects of SES on Overnight Hospitalization}

As was the case for the total sample, the direct association of employment status with overnight hospitalization was significant in the White group $\left(\beta_{\mathrm{job}}=-0.133, \mathrm{SE}=0.063\right.$, $\left.\beta_{\text {job }}^{*}=-0.056, p<0.05\right)$, indicating that being employed was associated with lower hospitalization rates controlling for other SES and demographic variables. All other adjusted direct SES to overnight hospitalization associations across race/ethnic groups were not significantly different from zero.

\subsubsection{Indirect Effects of SES on Overnight Hospitalization via Depressive Symptoms}

Results in the preceding sections indicated that several individual paths across all groups were weak or non-significant. Because the indirect effect was based on the assessment of the product of two paths, the product of coefficients indicating indirect relationship was likely to be small. As suspected, examination of the indirect paths revealed that none of the SES variables was indirectly associated with overnight hospitalization via depressive symptoms. While the effects of SES on depressive symptoms were different from zero in some cases, SES did not seem to be significantly indirectly related to overnight hospitalization via depressive symptoms in any race/ethnic group. 


\section{Summary}

In summary, the results failed to support hypothesis 5, indicating that, at least in this sample, SES was not indirectly related to overnight hospitalization via depressive symptoms across race/ethnic group. There was strong SES to depressive symptoms relationship and limited direct SES to overnight hospitalization across groups, however. The next model examined behavioral factors (BMI and alcoholic beverage consumption) as mediating variables in the SES to overnight hospitalization relationship.

\subsection{SES-Behavioral Factors-Overnight Hospitalization: Lagged Path Model}

Hypothesis 6: Each SES variable will be negatively related to overnight hospitalization, body mass index and alcoholic beverage consumption. Body mass index and alcoholic beverage consumption, in turn, will be positively related to overnight hospitalization. These paths will differ across racelethnic groups.

\section{Lagged Regression Path Model for Behavioral Factors Mediating Variables}

This section examines the indirect association of SES variables with overnight hospitalization via BMI and alcoholic beverage consumption. Body mass index and alcoholic beverage consumption were used as intermediate behavioral factors to examine whether SES was indirectly related to overnight hospitalization through BMI or alcoholic beverage consumption. If SES was indirectly related to overnight hospitalization, it was expected that increase in SES would be related to decrease in BMI and alcoholic beverage consumption, and in turn, decrease in BMI and alcoholic beverage consumption would be associated with decrease in rates of overnight hospitalization. 


\subsubsection{Path Model for the Total Sample}

Lagged multiple mediator path model was tested in the total sample to examine whether SES was indirectly associated with overnight hospitalization via BMI and alcoholic beverage consumption. The model fit to the data well, $\left[\left(\chi^{2}(5)=23.73, \mathrm{CFI}=1.00\right.\right.$, RMSEA=0.022]. Overall, results indicated that SES was negatively related to BMI providing evidence in favor of hypothesis 6 although the path coefficients were not significant. However, SES was positively related to alcoholic beverage consumption, contrary to the hypothesized relationships. Only, income $\left(\beta_{\mathrm{inc}}=0.011, \mathrm{SE}=0.005\right.$, $\left.\beta_{\text {inc }}^{*}=0.012, p<0.05\right)$ was significantly related to levels of alcoholic beverage consumption, suggesting that respondents with higher income tended to drink more alcoholic beverages. As expected, paths from $\mathrm{BMI}\left(\beta=0.014, \mathrm{SE}=0.006, \beta^{*}=0.064, \mathrm{p}\right.$ $<0.05)$ and alcohol consumption level $\left(\beta=0.076, \mathrm{SE}=0.018, \beta^{*}=0.078, \mathrm{p}<0.001\right)$ to overnight hospitalization were significant, suggesting that higher BMI and higher alcoholic beverage consumption were associated with higher rates of overnight hospitalization, controlling for SES and demographic variables.

\subsubsection{Multiple Group Path Model}

A multiple group, multiple mediator, lagged path model was tested to examine weather SES was indirectly associated with overnight hospitalization via BMI and alcohol beverage consumption. The model fit to the data well, $\left[\left(\chi^{2}(15)=27.61, \mathrm{CFI}=1.00\right.\right.$, RMSEA=0.018]. In the White group, the overall results indicated that SES was negatively related to BMI providing support to the hypothesis. Socioeconomic status variables to BMI path coefficients in the Black and Hispanic group were similar (i.e., 
signs of path coefficients) to paths in the White group. Socioeconomic status was positively related to alcoholic beverage consumption, contrary to the hypothesized relationship.

\subsubsection{Direct Effects of SES on BMI and Alcoholic Beverage Consumption}

Across race/ethnic groups, few SES variables were significantly related to BMI and alcoholic beverage consumption. In the White group, wealth $\left(\beta_{\mathrm{wlth}}=-0.027, \mathrm{SE}=0.01\right.$, $\left.\beta^{*}{ }_{\text {wlth }}=-0.019, \mathrm{p}<0.05\right)$ and education levels $\left(\beta_{\text {edu }}=-0.032, \mathrm{SE}=0.016, \beta^{*}{ }_{\text {edu }}=-0.016, \mathrm{p}\right.$ $<0.05)$ were significantly related to BMI. Increase in wealth was associated with small decrease in BMI and increase in education level was associated with decrease in BMI. In the Hispanic and Black groups, none of the SES to BMI path coefficients was significantly different from zero.

It was expected that each SES variable would be negatively related to alcoholic beverage consumption. Contrary to hypothesis 6 , the overall relationship between SES and alcoholic beverage consumption was positive, indicating, on average, higher SES individuals tended to drink more alcoholic beverages. In Whites, income $\left(\beta_{\text {inc }}=0.026\right.$, $\left.\mathrm{SE}=0.006, \beta^{*}{ }_{\text {inc }}=0.025, \mathrm{p}<0.001\right)$ and wealth $\left(\beta_{\mathrm{wlth}}=0.004, \mathrm{SE}=0.002, \beta^{*}{ }_{\text {wlth }}=0.012, \mathrm{p}\right.$ $<0.05)$ were significantly related to alcoholic beverage consumption, such that, increases in income and wealth was associated with increase in alcohol consumption levels. In Blacks, only education $\left(\beta_{\text {edu }}=0.08, \mathrm{SE}=0.004, \beta^{*}{ }_{\text {edu }}=0.038, \mathrm{p}<0.05\right)$ was significantly related to alcoholic beverage consumption, indicating that individuals with higher levels of education, on average, consumed higher alcoholic beverages. None of the SES to BMI, 
and SES to alcoholic beverage consumption paths was significantly different from zero in the Hispanic group.

As hypothesized, the paths from BMI $\left(\beta_{\mathrm{bmi}}=0.014, \mathrm{SE}=0.006, \beta^{*}{ }_{\mathrm{bmi}}=0.064, \mathrm{p}\right.$ $<0.001)$ and alcoholic beverage consumption $\left(\beta_{\mathrm{alc}}=0.076, \mathrm{SE}=0.018, \beta_{\text {alc }}^{*}=0.078, \mathrm{p}\right.$ $<0.001)$ to overnight hospitalization were significantly different from zero, suggesting that higher BMI and higher alcoholic beverage consumption were associated with increase in overnight hospitalization rates in the White group. In Blacks, only the BMI $\left(\beta_{\mathrm{bmi}}=-0.134, \mathrm{SE}=0.063, \beta_{\mathrm{bmi}}^{*}=-0.056, \mathrm{p}<0.001\right)$ to overnight hospitalization path coefficient was significant, indicating increase in BMI was also related to lower rates of overnight hospitalization in this group. In the Hispanic group, BMI to overnight hospitalization and alcoholic beverage consumption to overnight hospitalization paths were not significantly different from zero.

\subsubsection{Direct Effects of SES on Overnight Hospitalization}

Higher SES was, on average, expected to be related to lower overnight hospitalization. In general, results indicated that SES was negatively related to overnight hospitalization as hypothesized. Overall, path coefficients for the relationship from SES to overnight hospitalization did not differ across race/ethnic groups. In Whites, only employment status was significantly $\left(\beta_{\mathrm{job}}=-0.134, \mathrm{SE}=0.063, \beta^{*}{ }_{\mathrm{job}}=-0.056, \mathrm{p}<0.05\right)$ related to overnight hospitalization, controlling for other SES and demographic variables, suggesting that employment was related decreased overnight hospitalization rates in this group. In the Hispanic group, the adjusted income to overnight hospitalization path coefficient remained significant $\left(\beta_{\text {inc }}=-0.142, \mathrm{SE}=0.06, \beta^{*}{ }_{\text {inc }}=-0.207, \mathrm{p}<0.05\right)$, suggesting 
that Hispanics with higher incomes were less likely to be hospitalized overnight. But, none of the path coefficients for the relationships from SES to overnight hospitalization were significantly different from zero in the Black group.

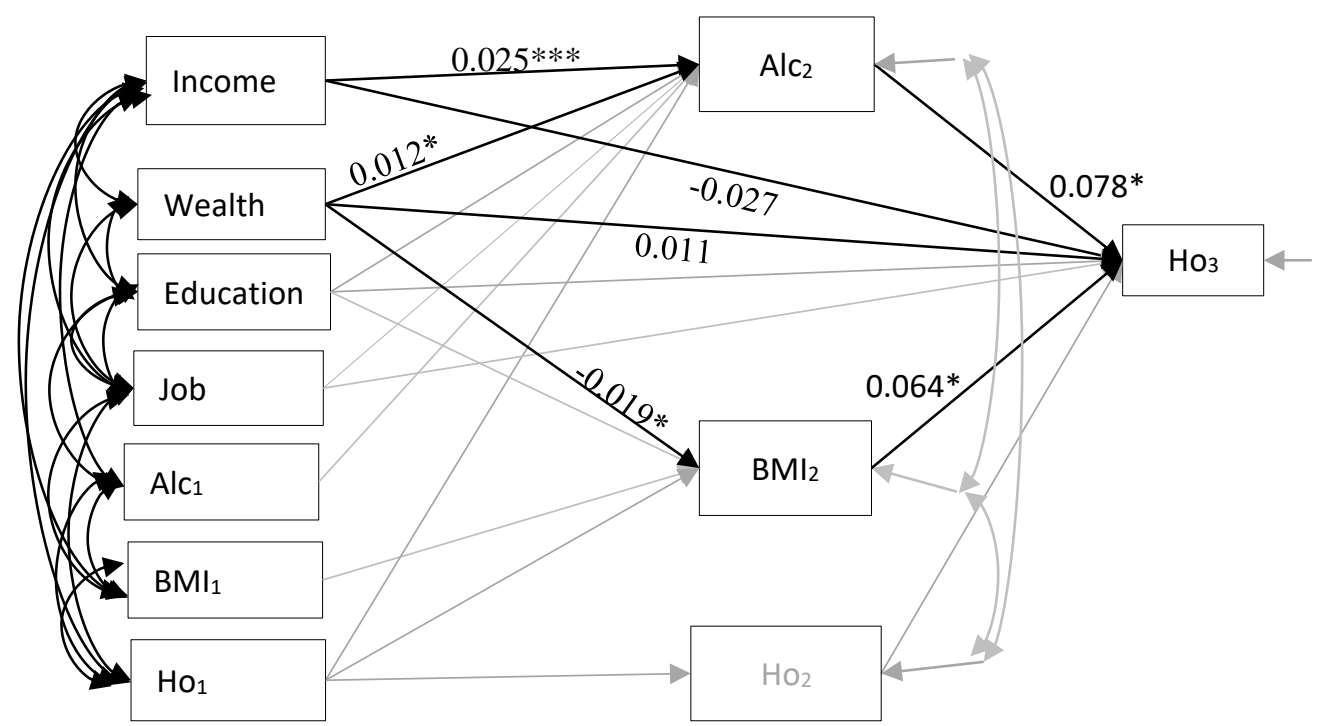

Figure 22: Lagged regression model for Whites $(\mathrm{N}=5717)$ with standardized path coefficients (Behavioral variable Mediators)

Note: $* \mathrm{p}<0.05 ; * * *<0.001$. Only paths pertinent to significant indirect relationships are shown. All control variables, paths modeled, variances and covariances are not shown to avoid clutter. Alc $=$ alcoholic beverage consumption, BMI=body mass index, $\mathbf{P I}=$ physical impairment, $\mathbf{H o =}=$ overnight hospitalization; subscripts indicate time points.

\subsubsection{Indirect Effects of SES on Overnight Hospitalization through Behavioral Variables}

As in all the models considered previously, the primary objective of hypothesis 6 was to examine the indirect associations of each SES variable with overnight hospitalization. Only in the White group did wealth and income indirectly relate to overnight hospitalization, whereas, none of the SES variables were indirectly associated with overnight hospitalization in the Hispanic and Black groups. 
In Whites, the indirect relationship between income and overnight hospitalization through alcoholic beverage consumption was significant $\left(\beta_{\mathrm{inc}}=0.002, \mathrm{SE}=0.001\right.$, $\left.\beta^{*}{ }_{\text {inc }}=0.002, \mathrm{p}<0.0 .05\right)$ but in the opposite direction. The income to overnight hospitalization path was negative failing to support the hypothesized relationship. Higher income was related to higher alcoholic beverage consumption and higher alcoholic beverage consumption, in turn, was related to higher overnight hospitalization rates. It is probable that income earning individuals had better access to medical services due to insurance coverage as well as ability to afford hospital expenses. Wealth $\left(\beta_{\mathrm{wlth}}=0.000\right.$, $\mathrm{SE}=0.00, \beta^{*}{ }_{\text {wlth }}=0.001, \mathrm{p}=0.061$ ) had similar indirect relationship to overnight hospitalization in the White group, indicating increase in wealth was associated with increase in overnight hospitalization via increase in alcoholic beverage consumption, contrary to the hypothesized relationships. It appeared, in this study, that wealthier Whites and high income earning Whites consumed higher alcoholic beverages. Additionally, the overall relationship of SES to drinking was positive across groups.

Considering the path via BMI in the White group, wealth $\left(\beta_{\mathrm{wlth}}=-0.000, \mathrm{SE}=0.00\right.$, $\left.\beta^{*}{ }_{\text {wlth }}=-0.001, \mathrm{p}<0.05\right)$ was significantly related to overnight hospitalization through BMI. The standardized path coefficient indicated that increase in wealth was associated with a tiny decrease in overnight hospitalization through decrease in BMI supporting the hypothesis. Note, however, that the effect sizes appeared to be quite small across groups. 


\section{Follow-up Analysis}

Multiple group path invariance tests, using nested models with paths from wealth to overnight hospitalization via body mass index were constrained across groups, were performed. Nested chi-square tests indicated that constraining these paths across groups did not significantly worsen fit $\left[\left(\Delta \chi^{2}(6)=5.25, \mathrm{~ns}\right)\right]$, suggesting the effect of wealth on overnight hospitalization via BMI was not different across groups.

\section{Summary}

In summary, the above results suggest that, in general, SES was related differently to alcoholic beverage use such that higher wealth and income was related to higher alcoholic beverage consumption contrary to the hypothesized relationships. But SES was inversely related to BMI consistent with the hypothesis. Some SES variables were significantly indirectly related to overnight hospitalization at least in the White group, supporting hypothesis 6 . Income was negatively correlated with overnight hospitalization but the income to alcoholic beverage consumption, and from alcoholic beverage consumption to overnight hospitalization path coefficients were positive. The sign of the product of these two coefficients was different from the sign of the direct path coefficient indicating a suppression effect. Therefore, the relationships of income to overnight hospitalization via alcoholic beverage as postulated in hypothesis 6 was not supported. This suggests that the alcoholic beverage consumption (drinking) variable, in a nationally representative sample, may not represent problem drinking. The effect sizes were small across groups, however. 


\section{Missing Data in SEM}

Survey data are prone to missing data issues; particularly, missing data problems are pervasive in longitudinal studies due to attrition in addition to various other reasons. The data used in this study were collected over ten years, covering five waves, and there were several missing values in the data across waves. The literature identifies three types of missing data mechanisms (missing completely at random [MCAR], missing at random [MAR], and missing not at random [MNAR]). The MAR assumption cannot be tested but some tests for MCAR assumption are available (Schafer \& Graham, 2002). A missing data pattern is a different concept than a missing data mechanism. Missing data mechanisms have to do with the probability of data being missing, whereas, missing data patterns are descriptive of the patterns of missing data, that is, the structure of missing scores and observed scores in a data set. Extensive discussions about missing data mechanisms and practical approaches to missing data analysis can be found elsewhere (Enders, 2010; Newsom, 2015; Rubin, 1976a; Schafer \& Graham, 2002). Most of the issues arising from missing data mechanisms are addressed by modern analytic software packages. Programs that implement maximum likelihood (ML) estimation techniques or use multiple imputation (MI) techniques provide unbiased estimates (Schafer \& Graham, 2002) even when some missing data assumptions (especially MAR assumptions) may be slightly violated. This study used ML estimation for missing data (i.e., full information maximum likelihood [FIML] in the presence of missing data) and when FIML is used, MAR is assumed, otherwise, estimates may be biased. Dropout patterns of respondents for the physical impairment outcome variable are provided below (Table 9) and patterns 
of missing data on the mediators (i.e., social integration, alcoholic beverage consumption, BMI, and the three factors for the depressive symptoms variable) are provided in Appendix B.

Table 9: Percent missing data for the physical impairment outcome variable (PI=physical impairment)

\begin{tabular}{|l|r|r|r|r|r|r|}
\hline & \multicolumn{2}{|c|}{ White $(\mathrm{N}=5717)$} & \multicolumn{2}{c|}{ Black $(\mathrm{N}=1238)$} & \multicolumn{2}{c|}{ Hispanic (N=719) } \\
\hline Time & Missing & \multicolumn{1}{c|}{$\%$} & \multicolumn{1}{c|}{ Missing } & \multicolumn{1}{l|}{$\%$} & \multicolumn{1}{c|}{ Missing } & \multicolumn{1}{l|}{} \\
\hline $\mathrm{T}=1$ & 627 & 11.0 & 153 & 12.4 & 95 & 13.2 \\
\hline $\mathrm{T}=2$ & 893 & 15.6 & 212 & 17.1 & 130 & 18.1 \\
\hline $\mathrm{T}=3$ & 1099 & 19.2 & 317 & 25.6 & 184 & 25.6 \\
\hline $\mathrm{T}=4$ & 1363 & 23.8 & 348 & 28.1 & 201 & 28.0 \\
\hline $\mathrm{T}=5$ & 1715 & 30.0 & 417 & 33.7 & 239 & 33.2 \\
\hline
\end{tabular}

Missing data increased monotonically in each race/ethnic group as indicated, reaching roughly $30 \%$ by the end of wave five $(\mathrm{T}=5)$ in each group. Nearly $65 \%$ of Whites, $56 \%$ of Blacks and $58 \%$ of Hispanics had complete data for five waves (indicated by +++++ in Table 10). Considering cases with missing values only at the last two waves, slightly over $75 \%$ of Whites, and nearly $70 \%$ of Blacks and Hispanics had complete data. Note that these data were collected over the span of 10 years. Assessment of correlations indicated that, overall, in Whites, Blacks and Hispanics, decrease in income, wealth, education, and unemployment were related to missing data, indicating that lower SES respondents were more likely to have missing data over time than those with higher SES at baseline. These correlations were not significant, however. Patterns of missing data for the physical impairment variable were similar across groups. 
Table 10: Patterns of missing data for physical impairment ('+' $=$ not missing)

\begin{tabular}{|c|c|c|c|c|}
\hline Race/ethnicity & Patterns & $\begin{array}{l}\text { Missing } \\
\text { values }\end{array}$ & Freq. & $\%$ \\
\hline \multirow{6}{*}{ White } & +++++ & 0 & 3700 & 64.7 \\
\hline & ++++ & 1 & 393 & 6.9 \\
\hline &..+++ & 2 & 279 & 4.9 \\
\hline & $++\ldots$ & 3 & 228 & 4.0 \\
\hline & $+\ldots$ & 4 & 250 & 4.4 \\
\hline & $\ldots \ldots$ & 5 & 445 & 7.8 \\
\hline \multirow{6}{*}{ Black } & +++++ & 0 & 688 & 55.6 \\
\hline & ++++ & 1 & 94 & 7.6 \\
\hline &..+++ & 2 & 67 & 5.4 \\
\hline & $++\ldots$ & 3 & 71 & 5.7 \\
\hline & $+\ldots$ & 4 & 72 & 5.8 \\
\hline & $\ldots \ldots$ & 5 & 76 & 6.1 \\
\hline \multirow{6}{*}{ Hispanic } & +++++ & 0 & 414 & 57.6 \\
\hline & ++++ & 1 & 54 & 7.5 \\
\hline &..+++ & 2 & 31 & 4.3 \\
\hline & $++\ldots$ & 3 & 45 & 6.3 \\
\hline & $+\ldots$ & 4 & 29 & 4.0 \\
\hline & $\ldots \ldots$ & 5 & 65 & 9.0 \\
\hline
\end{tabular}

Note: +++++ are for physical impairment at Time 1 to Time 5 respectively

The second major outcome variable was overnight hospitalization; a binary variable measured over three time points. Patterns of missing data, in this case also indicate a monotonic increase over three time points (covering six years). Similar to dropout of respondents in the physical impairment variable, Hispanics and Blacks had a slightly higher percent of missing data (Table 11).

Table 11: Percent missing data for the overnight hospitalization variable $(\mathrm{OH}=$ overnight hospitalization $)$

\begin{tabular}{|l|l|l|l|l|l|l|}
\hline & White $(\mathrm{N}=5717)$ & & \multicolumn{2}{|c|}{ Black (N=1238) } & \multicolumn{2}{|c|}{ Hispanic (N=719) } \\
\hline Time & Missing & $\%$ & Missing & $\%$ & Missing & $\%$ \\
\hline $\mathrm{T}=1$ & 900 & 15.7 & 200 & 16.2 & 135 & 18.8 \\
\hline $\mathrm{T}=2$ & 1162 & 20.3 & 267 & 21.6 & 171 & 23.8 \\
\hline $\mathrm{T}=3$ & 1381 & 24.2 & 369 & 29.8 & 223 & 31.0 \\
\hline
\end{tabular}


Table 12: Patterns of missing data for overnight hospitalization ('+' = not missing)

\begin{tabular}{|c|c|c|c|c|}
\hline Race/ethnicity & Pattern & $\begin{array}{c}\text { Missing } \\
\text { values }\end{array}$ & Freq. & $\%$ \\
\hline \multirow{8}{*}{ White } & +++ & 0 & 3961 & 69.3 \\
\hline & ++ & 1 & 377 & 6.6 \\
\hline &..+ & 2 & 339 & 5.9 \\
\hline &.++ & 1 & 140 & 2.4 \\
\hline &.++ & 1 & 139 & 2.4 \\
\hline &..+ & 2 & 96 & 1.7 \\
\hline &..+ & 2 & 78 & 1.4 \\
\hline & $\ldots$ & 3 & 587 & 10.3 \\
\hline \multirow{8}{*}{ Black } & +++ & 0 & 788 & 63.7 \\
\hline & ++ & 1 & 123 & 9.9 \\
\hline &..+ & 2 & 101 & 8.2 \\
\hline &.++ & 1 & 38 & 3.1 \\
\hline &.++ & 1 & 26 & 2.1 \\
\hline &.+ & 2 & 22 & 1.8 \\
\hline &..+ & 2 & 17 & 1.4 \\
\hline & $\ldots$ & 3 & 123 & 9.9 \\
\hline \multirow{8}{*}{ Hispanic } & +++ & 0 & 449 & 62.4 \\
\hline & ++ & 1 & 78 & 10.8 \\
\hline &..+ & 2 & 42 & 5.8 \\
\hline &..+ & 2 & 19 & 2.6 \\
\hline &.++ & 1 & 15 & 2.1 \\
\hline &.++ & 1 & 13 & 1.8 \\
\hline &.+ & 2 & 8 & 1.1 \\
\hline & $\ldots$ & 3 & 95 & 13.2 \\
\hline
\end{tabular}

Table 12 shows that attrition was the major cause for missing data, but the patterns also indicate arbitrary missing data patterns for small number of cases. Over $60 \%$ of the cases in each group had complete data, with the White group having nearly $70 \%$ complete data. More than $70 \%$ of the respondents in each group had complete data for the first and second time points. About $10 \%$ of cases in each group had missing data on all three waves. It appears, as was the case for the physical impairment variable, that there was sufficient sample in each group for the models. 


\section{CHAPTER 6.0: RESULTS FOR RECONSTRUCTABILITY ANALYSIS}

The Reconstructability Analysis (RA) part of the dissertation also focused on two outcome variables - physical impairment status (PI) and overnight hospitalization (Ho).

For longitudinal analysis, time between adjacent points was two years. Table 13 shows summary of predictive variables for the physical impairment variable following the conceptual model provided earlier (Figure 3, Chapter 3). In all groups, models were more complex in the cross-sectional analysis than in the longitudinal analysis. All models except presence of negative affect $\left(\checkmark^{*}\right)$ and absence of positive affect $\left(\checkmark^{*}\right)$ consisted of single predicting variables $(\checkmark) . \% \Delta \mathrm{U}(\mathrm{PI})$ indicates percent uncertainty reduction in physical impairment due to the predictive variables in the model.

Table 13: Cross-sectional/Longitudinal predictive variables for physical impairment (PI) status

\begin{tabular}{|c|c|c|c|c|c|c|c|c|c|c|c|}
\hline $\begin{array}{l}\mathbf{M} \\
\#\end{array}$ & $\begin{array}{l}\text { Cross- } \\
\text { Section. }\end{array}$ & $\begin{array}{c}\text { Job } \\
\text { status }\end{array}$ & $\begin{array}{l}\text { Som. } \\
\text { Symp }\end{array}$ & $\begin{array}{l}\text { Neg. } \\
\text { affect }\end{array}$ & $\begin{array}{l}\text { Posit. } \\
\text { affect }\end{array}$ & Exe & BMI & $\begin{array}{c}\text { Volunt } \\
\text { Orgs }\end{array}$ & $\% \Delta \mathrm{U}(\mathrm{PI})$ & $\Delta \mathrm{df}$ & $\mathrm{N}$ \\
\hline 1 & White & $\checkmark$ & $\checkmark$ & - & - & $\checkmark$ & - & $\checkmark$ & 18.6 & 6 & 511 \\
\hline 2 & Hispanic & $\checkmark$ & $\checkmark$ & - & $\checkmark$ & - & $\checkmark$ & - & 23.6 & 6 & 460 \\
\hline 3 & White & $\checkmark$ & $\checkmark$ & - & $\checkmark$ & $\checkmark$ & $\checkmark$ & $\checkmark$ & 20.3 & 9 & 865 \\
\hline 4 & Black & $\checkmark$ & $\checkmark$ & $\checkmark^{*}$ & $\checkmark^{*}$ & - & $\checkmark$ & - & 18.1 & 7 & 854 \\
\hline 5 & Black & $\checkmark$ & $\checkmark$ & $\checkmark$ & - & - & $\checkmark$ & - & 16.8 & 6 & 477 \\
\hline 6 & Hispanic & $\checkmark$ & $\checkmark$ & - & $\checkmark$ & - & $\checkmark$ & - & 23.6 & 6 & 460 \\
\hline & \multicolumn{9}{|c|}{ Longitudinal } & & \\
\hline 7 & White & - & - & - & - & $\checkmark$ & $\checkmark$ & $\checkmark$ & 10.9 & 5 & 408 \\
\hline 8 & Hispanic & - & $\checkmark$ & $\checkmark$ & - & - & - & - & 9.7 & 2 & 372 \\
\hline 9 & White & - & $\checkmark$ & - & - & $\checkmark$ & - & $\checkmark$ & 9.0 & 4 & 680 \\
\hline 10 & Black & $\checkmark$ & $\checkmark$ & $\checkmark$ & - & - & - & $\checkmark$ & 15.5 & 5 & 679 \\
\hline 11 & Black & - & $\checkmark$ & $\checkmark$ & - & - & $\checkmark$ & $\checkmark$ & 15.4 & 5 & 376 \\
\hline 12 & Hispanic & - & $\checkmark$ & $\checkmark$ & - & - & - & - & 9.7 & 2 & 372 \\
\hline
\end{tabular}

Note: $\checkmark$ predictive variables; $\checkmark^{*}$ jointly predictors. In longitudinal, SES \& $\{$ SPB $\}$ variables were from Time 2 and PI=Physical impairment from Time 3. Positive affect was reverse coded. Som Symp=somatic symptoms, Neg=Negative, Posit=Positive, Exe=Exercise, BMI=body mass index, Volunt Orgs=volunteering in organizations, \& $\mathbf{U}=$ uncertainty (entropy) reduction. $\mathbf{M}=$ Model. 
The following summarize some of the trends in Table 13 and provide main observations from fit analyses of these models:

- Models \#2 and \#6 were the same, as were Models \#8 and \#12, because Whites and Blacks were matched to available Hispanic data since the Hispanic group was the smallest.

- $\% \Delta \mathrm{U}(\mathrm{PI})$ in Model 10 is similar to $\% \Delta \mathrm{U}(\mathrm{PI})$ in model 11 . Three out of four of the variables are the same and the contribution of job status in uncertainty reduction in Model 10 may be similar to the contribution of BMI in Model 11.

- Job status and somatic symptoms were common in cross-sectional models across groups

- Somatic symptoms at the second time point predicted physical impairment status at the third time point, and job status dropped from all models except in one model for Blacks (Model 10) in the longitudinal analysis.

- Exercise and volunteering only appeared in models for the White group while negative affect only appeared in models for Blacks.

- Positive affect appears in Whites and Hispanics but not in Blacks.

- Job status drops out in longitudinal models but somatic symptoms variable remains. Exercise predicted physical impairment for Whites but didn't appear in models for other groups.

- In the longitudinal models, volunteering appeared in models for Whites and Blacks but not in models for the Hispanic group. Note that Volunteering also appeared in the cross-sectional models for the White group.

Fit results:

Composite IV states such as reporting somatic symptoms and not volunteering, or reporting somatic symptoms and being unemployed, raised risk of physical impairment. In general being employed, absence of somatic symptoms, presence of positive affect, 
absence of negative affect and exercising frequently or moderately frequently, and volunteering in organizations were associated with reduced risk of reporting physical impairment.

Cross-sectional paths:

- Selected SES variables were more strongly related to psychological variables (negative affect and somatic symptoms).

- These psychological variables, in turn, were stronger predictors of physical impairment status than social or behavioral variables

- All SES variables, except age, appeared in path models (see Tables 21 \& 30)

- For social variables, marital status was the most frequently related intermediate variable to SES variables.

- Income and gender often jointly predicted marital status but marital status was not strong predictor of physical impairment status.

\section{Longitudinal paths:}

- SES to mediator variable relationships were similar to paths for cross-sectional models

- Mediator variables strongly or moderately related to physical impairment status were somatic symptoms, negative affect and marital status.

\section{Overnight hospitalization}

- Few variables including gender and negative affect in Hispanics and job status in Blacks predicted overnight hospitalization.

- In Whites, the model failed to suggest any SES/demographic, social, psychological or behavioral variables that were related to overnight hospitalization. 
- Longitudinally, exercise and somatic symptoms at the second time point were related to overnight hospitalization at the third time point both in Blacks and Whites.

- Selected intermediate variables for models in path analysis were not strongly related to overnight hospitalization in cross-sectional models and longitudinal path models in all groups.

The next sections provide detailed analyses of cross-sectional and longitudinal models.

\section{Physical Impairment Status}

The outcome variable in this section was physical impairment status which was a binary variable $(0 / 1=$ absence/presence of difficulty in performing activities of daily living). Section 6.1 examines cross-sectional relationships with SES/demographic, social, psychological and behavioral variables as predictors. In section 6.2, intermediate (social, psychological or behavioral) variables associated with SES/demographic variables were selected and used in the indirect path from SES/demographic to physical impairment status. Percent uncertainty reductions (or path values) were computed comparing two race/ethnic groups at a time. Sections 6.3 and 6.4 replicate Sections 6.1 and 6.2, respectively, using social, psychological, and behavioral variables from the second time point and physical impairment variable from the third time point.

The focus was to examine differences by race/ethnicity given overlapping predictive variables. Brief discussions of differences due to non-overlapping predictor variables are provided in Appendix C along with RA fit results for the full models. 


\subsection{Cross-Sectional Analysis of Physical Impairment Status}

This section provides cross-sectional results where all six SES/demographic variables (collectively indicated by the letter $\mathbf{D}$ ), five social $(\mathbf{S})$ variables, three psychological (P) variables, and four behavioral (B) variables were simultaneously used to predict physical impairment (PI).

The research questions in this section were:

RQ1a: How do SES/demographic variables, social integration variables, psychological variables, and behavioral variables interact in predicting physical impairment status? RQ1b: Do such predictions differ by race/ethnic groups?

All models with loops for the cross-sectional comparison of race/ethnic groups are provided (Table 14). Models with loops are more fine-grained than models without loops. In this section, all variables in models without loops also appeared in models with loops and the models with loops had better BIC value. Therefore, only analyses of models with loops are provided. Thus, each of the models in Table 14 was fit to examine how composite IV states of predictive variables were associated with physical impairment status.

For these models the reference model was the independence model written as DSPB:PI where D, S, P and B were IVs representing multiple variables and PI was the DV representing single variable (PI=physical impairment). In short-hand the reference model (and the independence model in this case) is represented as IV: DV and best BIC model with predictors is given as IV:JobPI:SomaticPI:ExercisePI:VolunteerPI 
indicating job status, somatic symptoms, exercise and volunteering independently predicted physical impairment status (PI) (':' indicates independence). The 'IV’ part of the model in the Tables was dropped for simplicity but note that IV stands for all independent variables including the non-predictive variables and only predictive variables are included in the models below. (Note: in White vs Hispanic, upper model is for Whites, lower model for Hispanics; and similarly for other race/ethnic comparisons).

Table 14: Summary of results of inter-race/ethnic comparison of cross-sectional models

\begin{tabular}{|c|l|}
\hline Race/ethnicity & Model \\
\hline $\begin{array}{c}\text { White } \\
\text { vs } \\
\text { Hispanic }\end{array}$ & JobPI : SomaticPI : ExercisePI : VolunteerPI \\
\cline { 2 - 2 } & JobPI : SomaticPI : BmiH : PositivePI \\
\hline \hline $\begin{array}{c}\text { Vhite } \\
\text { Black }\end{array}$ & JobPI : SomaticPI : BmiPI : PositivePI : ExercisePI : VolunteerPI \\
\cline { 2 - 2 } $\begin{array}{c}\text { Black } \\
\text { vs } \\
\text { Hispanic }\end{array}$ & JobPI : SomaticNegativePI : BmiPI \\
\cline { 2 - 2 } & JobPI : SomaticPI : BmiPI : PositivePI \\
\hline \hline
\end{tabular}

* Psychological variables jointly predict physical impairment in Blacks

Note that in the analyses below, abbreviations of the variable names are used as follows: Job=J, Somatic $=$ Som, Exercise $=\mathbf{E x} ;$ Volunteer $=$ Vo; Bmi $=\mathbf{B m i} ;$ Positive $=$ Pos; Negative $=$ Neg .

Note: Positive affect was reverse coded. Bmi=body mass index

\section{(a) Whites versus Hispanics}

Several variables independently predicted physical impairment status in both Hispanics and Whites (Table 15). However, out of the four predicting variables, only two variables, employment status (J) and somatic symptoms (Som) appeared in both the Hispanic and White group models. Physical activity (Ex) and volunteering in organizations (Vo) were associated with physical impairment only in the White group, while body mass index (Bmi) and absence of positive affect were related to physical impairment status only in the Hispanic group. 
Percent uncertainty reduction in physical impairment status due to the four independently predicting variables was larger in the Hispanic group model although both models were equally complex. This probably indicated the importance of the two variables in the Hispanic model that did not appear in the model for Whites. Note that variables in bold also appeared in models without loops.

Table 15: Models with loops for White and Hispanic groups

\begin{tabular}{|l|l|l|l|}
\hline Race/ethnicity & \multicolumn{1}{|c|}{ Model } & $\Delta \mathrm{df}$ & $\% \Delta \mathrm{U}(\mathrm{PI})$ \\
\hline White & JPI:SomPI:ExPI:VoPI & 6 & 18.6 \\
\hline Hispanic & JPI:SomPI:BmiPI:PosPI & 6 & 23.6 \\
\hline
\end{tabular}

Note: variables in bold appear in models without loops.

$\mathrm{J}=$ job status; Som=Somatic symptoms; Ex=Exercise; Vo=Volunteering; Bmi=Body mass index; Pos=Positive affect

These models were further explored by fitting and examining composite IV states that increased or decreased risk of physical impairment (Table 16). Somatic symptoms and physical impairment status were binary variables $(0 / 1=$ absence/presence $)$ but job status had three categories, namely employed $(\mathrm{J}=1)$, retired $(\mathrm{J}=2)$ and 'other' $(\mathrm{J}=3)$. The 'other' job category included the homemaker, those on sick leave, those looking for job and the disabled. In both White and Hispanic groups, several predictor states appeared to reduce or increase risk of physical impairment relative to the marginal risk. 
Table 16: Fit results for JPI:SomPI for White and Hispanic groups

\begin{tabular}{|c|c|c|c|c|c|c|c|}
\hline \multirow[t]{2}{*}{ Race/ethnicity } & \multicolumn{2}{|r|}{ IV } & \multirow{2}{*}{$\begin{array}{l}\text { Data } \\
\text { freq }\end{array}$} & \multicolumn{4}{|c|}{$\begin{array}{l}\text { Model } \\
\text { calc. q(DV|IV) }\end{array}$} \\
\hline & $\mathrm{J}$ & Som & & $\mathrm{PI}=0$ & $\mathrm{PI}=1$ & $\mathrm{p}($ margin $)$ & Ratio, $\mathrm{PI}=1$ \\
\hline \multirow{7}{*}{ White } & ${ }^{\alpha} 1$ & 0 & 71 & 98.0 & 2.0 & 0.001 & 0.12 \\
\hline & 1 & 1 & 32 & 92.0 & 8.0 & 0.175 & 0.47 \\
\hline & $\alpha_{2}$ & 0 & 125 & 93.4 & 6.6 & 0.002 & 0.39 \\
\hline & 2 & 1 & 121 & 77.1 & 22.9 & 0.084 & 1.35 \\
\hline & 3 & 0 & 63 & 86.8 & 13.2 & 0.420 & 0.78 \\
\hline & $\beta_{3}$ & 1 & 99 & 60.9 & 39.1 & 0.000 & 2.30 \\
\hline & \multicolumn{3}{|c|}{ Marginal probability } & 83.0 & 17.0 & & \\
\hline \multirow{7}{*}{ Hispanic } & ${ }^{\alpha} 1$ & 0 & 59 & 98.6 & 1.4 & 0.000 & 0.08 \\
\hline & 1 & 1 & 36 & 91.2 & 8.8 & 0.121 & 0.46 \\
\hline & $\alpha_{2}$ & 0 & 111 & 94.8 & 5.2 & 0.000 & 0.28 \\
\hline & $\$ 2$ & 1 & 101 & 73.1 & 26.9 & 0.041 & 1.42 \\
\hline & 3 & 0 & 51 & 89.5 & 10.5 & 0.124 & 0.55 \\
\hline & $\beta_{3}$ & 1 & 102 & 56.2 & 43.8 & 0.000 & 2.32 \\
\hline & \multicolumn{3}{|c|}{ Marginal probability } & 81.1 & 18.9 & & \\
\hline
\end{tabular}

Note: ${ }^{\alpha}$ decrease risk $\&{ }^{\beta}$ increase risk both groups; ${ }^{\ddagger}$ increases risk in Hispanics only.

\section{Protective composite IV states}

Two composite IV states (rows in blue or ${ }^{\alpha}$ rows) had similar protective effect in Whites and Hispanics. Employed $(\mathrm{J}=1)$ Whites and Hispanics without somatic symptoms $(\mathrm{Som}=0)$ had lower risk of having physical impairment compared to their respective samples. The relative risk ratios of these composite IV states to the margin were 0.12 and 0.08, for Whites and Hispanics respectively, indicating the near absence of physical impairment in employed individuals who did not report somatic symptoms. Similarly, for retired $(\mathrm{J}=2)$ Whites and Hispanics who did not report somatic symptoms, risk of physical impairment decreased relative to the marginal risk in each sample.

\section{Risky composite IV states}

Common composite IV states (Table 16 , rows in red or ${ }^{\beta}$ rows) in the White and Hispanic groups that elevated the risk for physical impairment were presence of somatic 
symptoms (Som=1) and falling in job category 'other' $(\mathrm{J}=3)$. Whites and Hispanics other than retired or employed, and who experienced somatic symptoms were at significantly higher risk of having physical limitations. In fact, the respective risks for individuals with these composite IV states were more than two times higher than the marginal risk in the respective groups (2.30 for Whites and 2.32 for Hispanics).

There was one unique composite IV states (Table 16, ${ }^{\ddagger}$ row) increased risk of physical impairment in the Hispanic group but not in the White group. Retired Hispanics $(\mathrm{J}=2)$, with somatic symptoms (Som=1), had higher risk of reporting physical impairment compared to the marginal risk for that group. However, the relative risk ratios for Whites (1.35) and for Hispanics (1.42) with these composite IV states were similar, but were under and over the traditional 0.05 cutoff for significance level in Hispanics and Whites respectively.

\section{Summary}

Risky and protective composite IV states given the overlapping variables (job status and somatic symptoms) for Whites and Hispanics were similar with one exception. Retired Hispanics reporting somatic symptoms had significantly higher risk of reporting physical impairment relative to the marginal risk for the whole group, which was not the case in the White group. Factors affecting retirement decisions may vary by race/ethnicity. For both groups, having somatic symptoms was, in general, strongly associated with presence of some form of physical limitation. 


\section{(b) Whites versus Blacks}

The models with loops for both groups were relatively complex compared to the previous models. Several variables independently predicted physical impairment status in Whites and Blacks with large (20.3\% and $18.1 \%$ respectively) percent uncertainty reduction in physical impairment (Table 17). Job status and somatic symptoms variables in bold indicate that these variables also appeared in models without loops.

The model for Blacks had an interaction term where somatic symptoms and negative affect jointly predicted physical impairment status. The interaction effect should not be surprising because negative affect and somatic symptoms are sub-scales of a known depressive symptoms measure (CES-D scale) widely used in epidemiological studies as described in the SEM part of this dissertation. The fact that this interaction term did not appear in the model for Whites was notable, however.

Table 17: Models with loops for White and Black groups

\begin{tabular}{|l|l|c|c|}
\hline Race/ethnicity & Model & $\Delta \mathrm{df}$ & $\% \Delta \mathrm{U}(\mathrm{PI})$ \\
\hline White & JPI:BmiPI:SomPI:ExPI:PosPI:VoPI & 9 & 20.3 \\
\hline Black & JPI:BmiPI:SomNegPI & 7 & 18.1 \\
\hline
\end{tabular}

Note: variables in bold also appear in models without loops

A model only with common predictors (i.e., JPI:BmiPI:SomPI) was fit (Table 18) to further investigate the nature of the relationships at a more refined level by investigating composite IV states that reduced or increased risk of physical impairment. 


\section{Protective composite IV states}

There were three identical composite IV states that were protective in both Whites and Blacks (Table 18, rows in blue or ${ }^{\alpha}$ rows). The salient feature in these protective composite IV states was absence of somatic symptoms $(\mathrm{Som}=0)$ and not falling in job category 'other'. Excluding job category 'other' $(\mathrm{J}=3)$, for any combinations of the job status and body mass index status, and given that somatic symptoms were absent $($ Som $=0)$, Whites and Blacks had lower risk of physical impairment relative to their respective marginal risks.

Protective composite IV state in Whites was being employed $(\mathrm{J}=1)$, in combination with having normal weight $(\mathrm{Bmi}=1)$ and absence of somatic symptoms $($ Som $=0)$. These composite IV states in the model for Blacks also reduced risk of physical impairment, although due to small cell frequency, the significance level was over the traditional 0.05 cutoff point. The relative risk ratios for Blacks (0.13) and Whites $(0.19)$ with these composite IV states relative to the margin were low. Unique protective composite IV states in Blacks was being employed together with being overweight/obese $(\mathrm{Bmi}=2)$, and presence of somatic symptoms $(\mathrm{Som}=1)$. This was not the case in Whites, however. It appears, then, that being employed was an important protective state in Blacks as speculated earlier. Note that the first two $\left(1^{\text {st }} \& 2^{\text {nd }}\right.$ rows in Table 18 , the part for Blacks) composite IV states that included employment $(\mathrm{J}=1)$ and were not protective in the Black group had low cell counts. 
Table 18: Fit results for JPI:BmiPI:SomPI for White and Black groups

\begin{tabular}{|c|c|c|c|c|c|c|c|c|}
\hline \multirow[t]{2}{*}{ Race/ethnicity } & \multicolumn{3}{|c|}{ IV } & \multirow{2}{*}{$\begin{array}{c}\text { Data } \\
\text { freq }\end{array}$} & \multicolumn{2}{|c|}{$\begin{array}{c}\text { Model } \\
\text { calc. } \mathrm{q}(\mathrm{DV} \mid \mathrm{IV})\end{array}$} & \multirow[b]{2}{*}{$\mathrm{p}$ (margin) } & \multirow[b]{2}{*}{ Ratio, $\mathrm{PI}=1$} \\
\hline & $\mathrm{J}$ & Bmi & Som & & $\mathrm{PI}=0$ & $\mathrm{PI}=1$ & & \\
\hline \multirow{13}{*}{ White } & $\lambda_{1}$ & 1 & 0 & 41 & 96.7 & 3.3 & 0.017 & 0.19 \\
\hline & 1 & 1 & 1 & 25 & 87.8 & 12.2 & 0.479 & 0.69 \\
\hline & ${ }^{\alpha} 1$ & 2 & 0 & 82 & 95.2 & 4.8 & 0.002 & 0.27 \\
\hline & 1 & 2 & 1 & 45 & 83.0 & 17.0 & 0.902 & 0.96 \\
\hline & $\alpha_{2}$ & 1 & 0 & 75 & 95.0 & 5.0 & 0.004 & 0.28 \\
\hline & 2 & 1 & 1 & 68 & 82.5 & 17.5 & 0.965 & 0.99 \\
\hline & ${ }^{\alpha} 2$ & 2 & 0 & 166 & 92.8 & 7.2 & 0.000 & 0.41 \\
\hline & $\beta_{2}$ & 2 & 1 & 153 & 76.2 & 23.8 & 0.048 & 1.35 \\
\hline & 3 & 1 & 0 & 23 & 87.5 & 12.5 & 0.523 & 0.71 \\
\hline & $\beta_{3}$ & 1 & 1 & 44 & 63.4 & 36.6 & 0.001 & 2.07 \\
\hline & 3 & 2 & 0 & 41 & 82.6 & 17.4 & 0.963 & 0.98 \\
\hline & $\beta_{3}$ & 2 & 1 & 102 & 54.0 & 46.0 & 0.000 & 2.60 \\
\hline & \multicolumn{4}{|c|}{ Marginal probability } & 82.3 & 17.7 & & \\
\hline \multirow{13}{*}{ Black } & 1 & 1 & 0 & 17 & 97.4 & 2.6 & 0.074 & 0.13 \\
\hline & 1 & 1 & 1 & 16 & 90.6 & 9.4 & 0.307 & 0.47 \\
\hline & ${ }^{\alpha} 1$ & 2 & 0 & 105 & 97.4 & 2.6 & 0.000 & 0.13 \\
\hline & $\lambda_{1}$ & 2 & 1 & 56 & 90.6 & 9.4 & 0.05 & 0.47 \\
\hline & $\alpha_{2}$ & 1 & 0 & 49 & 91.4 & 8.6 & 0.047 & 0.43 \\
\hline & 2 & 1 & 1 & 59 & 73.4 & 26.6 & 0.201 & 1.33 \\
\hline & $\alpha_{2}$ & 2 & 0 & 170 & 91.4 & 8.6 & 0.000 & 0.43 \\
\hline & $\beta_{2}$ & 2 & 1 & 189 & 73.3 & 26.7 & 0.020 & 1.34 \\
\hline & 3 & 1 & 0 & 7 & 82.0 & 18.0 & 0.908 & 0.91 \\
\hline & $\beta_{3}$ & 1 & 1 & 26 & 54.2 & 45.8 & 0.001 & 2.30 \\
\hline & 3 & 2 & 0 & 42 & 81.9 & 18.1 & 0.771 & 0.91 \\
\hline & $\beta_{3}$ & 2 & 1 & 118 & 54.0 & 46.0 & 0.000 & 2.31 \\
\hline & \multicolumn{4}{|c|}{ Marginal probability } & 80.1 & 19.9 & & \\
\hline
\end{tabular}

Note: ${ }^{\alpha}=$ protective $\&^{\beta}=$ risky states in both groups; ${ }^{\lambda}=$ unique protective states in both groups

\section{Risky composite IV states}

Three identical composite IV states increased risk in Whites and Blacks (rows in red or ${ }^{\beta}$ rows). Here, the salient feature in the common risky predictor states was presence of somatic symptoms (Som=1). Retired ( $\mathrm{J}=2)$ Whites and Blacks who were overweight $(\mathrm{Bmi}=2)$ and reported somatic symptoms $(\mathrm{Som}=1)$ had higher risk of reporting physical impairment compared to the marginal risk for the respective groups. Regardless of BMI status, in both Whites and Blacks, being in job category 'other' and presence of somatic symptoms posed higher risk of physical impairment relative to the marginal risk in each 
group. Composite IV states that increased risk in both groups were, once again, falling in job category 'other' $(\mathrm{J}=3)$ and presence of somatic symptoms $(\mathrm{Som}=1)$, where, the risk for individuals with these states was more than two times the respective marginal risks for the samples.

\section{Summary}

There was a clear pattern in the variables that appeared in models with loops and models without loops for both race/ethnic groups. Job status (J) and somatic symptoms (Som) featured prominently in models with loops and without loops for both groups. The worst states given the overlapping variables were falling in job category 'other' $(\mathrm{J}=3)$ and presence of somatic symptoms (Som=1). When these states (i.e., J=3 \& Som=1) occurred together, irrespective of BMI status, the risk for physical impairment significantly increased in both groups. Protective states were, in general, being employed and absence of somatic symptoms. Being employed seemed to be more protective for Blacks and BMI status did not seem to matter in general but for Blacks in particular.

\section{(c) Blacks versus Hispanics}

Table 19 provides models with loops for Blacks and Hispanics. Both models were equally complex $(\Delta \mathrm{df}=6)$ but percent uncertainty reduction in physical impairment status was larger in the Hispanic group. Each model had one unique predictive variable that was not present in the other; negative affect (Neg) predicted physical impairment only in Blacks while positive affect (Pos) predicted physical impairment only in the Hispanic group. The discrepancy in percent uncertainty reduction in physical impairment given the 
equally complex models, therefore, could be due to positive affect being more informative of physical impairment status in Hispanics than negative affect was of physical impairment in Blacks.

Table 19: Models with loops for Black and Hispanic groups

\begin{tabular}{|l|l|c|c|}
\hline Race/ethnicity & \multicolumn{1}{|c|}{ Model } & $\Delta \mathrm{df}$ & $\% \Delta \mathrm{U}(\mathrm{PI})$ \\
\hline Black & JPI: SomPI:BmiPI:NegPI & 6 & 16.8 \\
\hline Hispanic & JPI: SomPI:BmiPI:PosPI & 6 & 23.6 \\
\hline
\end{tabular}

A model with only the common predicting variables (i.e., JPI:BmiPI:SomPI) was fit in each group and results are examined below.

\section{Protective composite IV states}

Two composite IV states (Table 20 , rows in blue or ${ }^{\alpha}$ rows) in each race/ethnic group were related to lower risk relative to the marginal risk to the respective total sample. Given that a respondent was employed $(\mathrm{J}=1)$ or retired $(\mathrm{J}=2)$, and was overweight $(\mathrm{Bmi}=2)$, and did not report somatic symptoms $(\mathrm{Som}=0)$, the risk of physical impairment $(\mathrm{PI}=1)$ was significantly lower than the marginal risk in each group even though BMI was high. Absence of somatic symptoms and not falling in job category 'other' $(\mathrm{J}=3)$, once again, seemed to play a key role in these protective IV states in both groups.

Although due to low cell frequency, the risk of physical impairment given the composite IV states of being employed $(\mathrm{J}=1)$ in combination with having normal weight $(\mathrm{Bmi}=1)$ and absence of somatic symptoms $(\mathrm{Som}=0)$ appeared to be similar to the marginal risk based on a 0.05 cutoff point for significance level, the 0.14 risk ratio for 
Blacks and 0.06 for Hispanics indicated that the respective composite IV states, indeed, reduced risk of physical impairment in both groups.

Table 20: Fit results for JPI:BmiPI:SomPI for Black and Hispanic groups

\begin{tabular}{|c|c|c|c|c|c|c|c|c|}
\hline \multirow[t]{2}{*}{ Race/ethnicity } & \multicolumn{3}{|c|}{ IV } & \multirow{2}{*}{$\begin{array}{l}\text { Data } \\
\text { freq }\end{array}$} & \multicolumn{2}{|c|}{$\begin{array}{c}\text { Model } \\
\text { calc. } \mathrm{q}(\mathrm{DV} \mid \mathrm{IV})\end{array}$} & & \\
\hline & $\mathrm{J}$ & Bmi & Som & & $\mathrm{PI}=0$ & $\mathrm{PI}=1$ & $\mathrm{p}($ margin $)$ & Ratio, $\mathrm{PI}=1$ \\
\hline \multirow{13}{*}{ Black } & 1 & 1 & 0 & 7 & 96.9 & 3.1 & 0.214 & 0.14 \\
\hline & 1 & 1 & 1 & 9 & 89.2 & 10.8 & 0.391 & 0.47 \\
\hline & ${ }^{\alpha} 1$ & 2 & 0 & 49 & 96.7 & 3.3 & 0.001 & 0.14 \\
\hline & 1 & 2 & 1 & 28 & 88.6 & 11.4 & 0.149 & 0.50 \\
\hline & 2 & 1 & 0 & 30 & 90.9 & 9.1 & 0.073 & 0.40 \\
\hline & 2 & 1 & 1 & 43 & 72.7 & 27.3 & 0.487 & 1.19 \\
\hline & $\alpha_{2}$ & 2 & 0 & 78 & 90.5 & 9.5 & 0.005 & 0.42 \\
\hline & 2 & 2 & 1 & 106 & 71.6 & 28.4 & 0.175 & 1.24 \\
\hline & 3 & 1 & 0 & 4 & 82.2 & 17.8 & 0.810 & 0.78 \\
\hline & $\$ 3$ & 1 & 1 & 17 & 55.1 & 44.9 & 0.030 & 1.96 \\
\hline & 3 & 2 & 0 & 23 & 81.4 & 18.6 & 0.629 & 0.81 \\
\hline & $\beta_{3}$ & 2 & 1 & 83 & 53.8 & 46.2 & 0.000 & 2.02 \\
\hline & \multicolumn{4}{|c|}{ Marginal probability } & 77.1 & 22.9 & & \\
\hline \multirow{13}{*}{ Hispanic } & 1 & 1 & 0 & 18 & 98.8 & 1.2 & 0.055 & 0.06 \\
\hline & 1 & 1 & 1 & 9 & 92.8 & 7.2 & 0.368 & 0.38 \\
\hline & ${ }^{\alpha} 1$ & 2 & 0 & 41 & 98.4 & 1.6 & 0.004 & 0.08 \\
\hline & 1 & 2 & 1 & 27 & 90.7 & 9.3 & 0.202 & 0.49 \\
\hline & $\lambda_{2}$ & 1 & 0 & 28 & 95.7 & 4.3 & 0.049 & 0.23 \\
\hline & 2 & 1 & 1 & 21 & 77.4 & 22.6 & 0.663 & 1.20 \\
\hline & $\alpha_{2}$ & 2 & 0 & 83 & 94.4 & 5.6 & 0.002 & 0.30 \\
\hline & $\$ 2$ & 2 & 1 & 80 & 72.1 & 27.9 & 0.039 & 1.48 \\
\hline & 3 & 1 & 0 & 17 & 91.4 & 8.6 & 0.280 & 0.46 \\
\hline & 3 & 1 & 1 & 15 & 62.0 & 38.0 & 0.059 & 2.01 \\
\hline & 3 & 2 & 0 & 34 & 88.9 & 11.1 & 0.248 & 0.59 \\
\hline & $\beta_{3}$ & 2 & 1 & 87 & 55.1 & 44.9 & 0.000 & 2.37 \\
\hline & \multicolumn{4}{|c|}{ Marginal probability } & 81.1 & 18.9 & & \\
\hline
\end{tabular}

Note: ${ }^{\alpha}=$ protective $\&{ }^{\beta}=$ risky states in both groups; ${ }^{\lambda}=$ unique protective states; ${ }^{\ddagger}=$ unique risky states

Another protective composite IV states in Hispanics but not in Blacks was being retired $(\mathrm{J}=2)$, having normal weight $(\mathrm{Bmi}=1)$ and absence of somatic symptoms $(\mathrm{Som}=0)$ (Table 20, row in green or ${ }^{\lambda}$ rows), which was lower than the marginal risk to the total sample in that group. The corresponding composite IV state for Blacks was only slightly better than the marginal risk to the group, further underlining the importance of being 
employed in Blacks. However, risk ratios of 0.40 and 0.23 for Blacks and Hispanics, respectively, were both small suggesting the lack of statistical significance to likely be due to low cell frequency in the Black group.

Risky composite IV states

Common composite IV states in Hispanics and Blacks (rows in red or ${ }^{\beta}$ rows) that increased risk of physical impairment were falling in job category 'other' $(\mathrm{J}=3)$, being overweight $(\mathrm{Bmi}=2)$ and presence of somatic symptoms $(\mathrm{Som}=1)$. The risk to individuals falling in these predictor states was more than two times the marginal risk to the respective groups. Note also that, overall, marginal risk for Blacks was higher than marginal risk for Hispanics (22.9 compared to 18.9).

Each group had one unique composite IV states that increased risk ( ${ }^{\ddagger}$ rows). In Blacks, falling in job category 'other' $(\mathrm{J}=3)$, having normal weight $(\mathrm{Bmi}=1)$ and presence of somatic symptoms (Som=1) increased risk relative to the marginal risk for the group. The corresponding composite IV states in Hispanics also increased risk as indicated by the higher risk ratio (2.01) although, due to low cell count, the significance level of the composite IV states compared to the margin was slightly over the 0.05 cutoff point. Unique composite IV states that posed greater risk in Hispanics compared to the marginal risk to the sample was being retired $(\mathrm{J}=2)$, being overweight $(\mathrm{Bmi}=2)$ and presence of somatic symptoms $(\mathrm{Som}=1)$. For Hispanics with this particular composite IV states, the risk of physical impairment increased by $48 \%$ relative to the margin. 


\section{Summary}

Similar to results in previous analyses comparing Whites with Blacks, BMI did not seem to play a critical role in predicting physical impairment in the above analyses. Being in job category 'other' and presence of somatic symptoms were related to higher risk of physical impairment. Risk of physical impairment for employed individuals $(\mathrm{J}=1)$, with normal weight $(\mathrm{Bmi}=1)$ and without somatic symptoms $(\mathrm{Som}=0)$ was low but due to low cell counts the significance level was over the traditional 0.05 cutoff point.

\subsection{Cross-Sectional Path Analysis of Physical Impairment Status}

This section (based on Figure 4, Chapter 3) provides path models for the physical impairment outcome variable using cross-sectional data. It compares uncertainty reductions (path values) in the intermediate variable due to the related SES variable(s) and the outcome variable due to the selected intermediate variable, by race/ethnic groups and examines possible indirect relationships between SES/demographic variable(s) and physical impairment status.

The research questions in this section were:

RQ2a: Do relationships between SES/demographic variables and intermediate variables differ by race/ethnicity?

RQ2b: Do relationships between intermediate variables and physical impairment status differ by race/ethnicity?

The analysis involved two main steps. First, a neutral search to determine D variables related to $\mathrm{S}$ (also to $\mathrm{P} \& \mathrm{~B}$ ) variables was conducted. Recall that D represents 
six SES/demographic variables, $\mathrm{S}$ represents five social variables, $\mathrm{P}$ represents three psychological variables, and B represents four behavioral variables. The $S_{i}$ variable that was associated with any $\mathrm{D}_{i}$ variables (where ${ }_{i}$ simply indexes selected variables from $\mathrm{D}$ and S) was used in the indirect path from D to PI (physical impairment). Further, only the $\mathrm{S}_{i}$ variable and $\mathrm{D}_{i}$ variable(s) associated with it were used in modeling (i.e., $\mathrm{D}$ variables not related to $S_{i}$ were excluded in directed path modeling).

The second step involved directed modeling to quantify and compare, by race/ethnic group, the two individual paths $\alpha$ and $\beta^{\prime}$ shown in Figure 23. Where SES/demographic variables and social variables (also psychological and behavioral variables) were different for race/ethnic groups, results were simply provided for each group. The dashed paths (dashed arrows to PI) denote that the effect of the intermediate variable $\mathrm{S}$ (also $\mathrm{P}$ and $\mathrm{B}$ ) was controlled for the effect of the SES/demographic variable(s) and, in turn, the effect of the specific SES/demographic variable(s) was controlled for the effect of the intermediate variable. The SES/demographic to intermediate path effects were estimated without adjusting for other variables ( $\alpha$ path). All uncertainty reductions are given in percent. Note that, for these path values, in RA (as in log-linear) modeling one cannot multiply a $\mathrm{D} \rightarrow \mathrm{S}$ path value $(\alpha)$ and $\mathrm{S} \rightarrow$ PI path value $\left(\beta^{\prime}\right)$ to get a net indirect $\mathrm{D} \rightarrow \mathrm{PI}$ path value. These procedures apply to all path models in this part of the dissertation. 


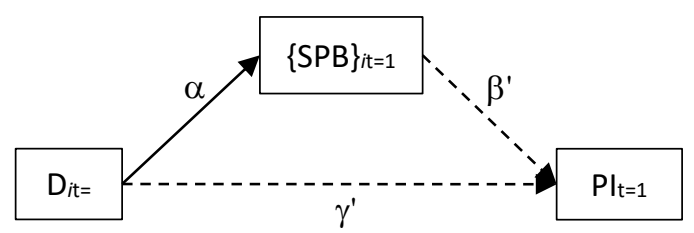

Figure 23: Cross-sectional path modeling of physical impairment status

Note: $\mathrm{i}=$ selected variable(s) \& $\mathrm{t}=$ time

Following the illustrations in Figure 23, the corresponding values for $\alpha, \beta^{\prime}$ and $\gamma^{\prime}$, where ' indicates that the $\beta$ path controls for the $\gamma$ and vice versa, are provided in Table 21. Using neutral search, that is, a search for associations between SES and each of the intermediate variables (P, B and S) identified $\mathrm{P}, \mathrm{B}, \mathrm{S}$ variables strongly associated with SES variables. The selected $\mathrm{P}, \mathrm{B}$, and $\mathrm{S}$ variables were used in the indirect path from SES to physical health status (these are given in $\beta^{\prime}$ column). The intermediate to health status path was adjusted for the direct effect of SES on the health variable. Similarly, the direct effect of SES on health status was adjusted for the effect of the intermediate variable. Table 21 provides the three path values that correspond to the paths in Figure 23.

As a rule of thumb, $8 \%$ uncertainty reduction is considered substantial. In this study, a percent uncertainty reduction less than $3.5 \%$ was disregarded, $3.5-4.9 \%$ was considered weak, $4.9-6.9 \%$ was considered moderate and greater than or equal to $6.9 \%$ was considered strong. The emphases of these analyses were on the paths from $\mathrm{D}$ to $\{\mathbf{S P B}\}$ and from $\{\mathbf{S P B}\}$ to PI comprising the indirect paths. There were 8 instances where the selected intermediate variable achieved at least 3.5\% uncertainty reductions (see $\boldsymbol{\beta}^{\prime}$ column for path values, Table 21) in the outcome variable (physical impairment). Notice that none of these paths involved intermediate behavioral variables. 
Table 21: Uncertainty reductions for cross-sectional path models of physical impairment status

\begin{tabular}{|c|c|c|c|c|}
\hline Race/ethnicity & $\{$ SPB $\}$ factors & $\alpha=\mathrm{T}(\mathrm{D}:\{\mathrm{SPB}\})$ & $\boldsymbol{\beta}^{\prime}=\mathrm{T}_{\mathrm{D}}(\{\mathrm{SPB}\}: \mathrm{PI})$ & $\gamma^{\prime}=\mathrm{T}_{\{\mathrm{SPB}\}}(\mathrm{D}: \mathrm{PI})$ \\
\hline \multirow{10}{*}{$\begin{array}{l}\text { White } \\
\text { vs } \\
\text { Hispanic }\end{array}$} & \multirow{3}{*}{ Social } & GI $\rightarrow$ Mar & Mar $\rightarrow$ PI & GI $\rightarrow$ PI \\
\hline & & 15.3 & 2.1 & 5.0 \\
\hline & & 12.4 & 3.5 & 5.4 \\
\hline & \multirow{3}{*}{ Psychological } & $\mathrm{W} \rightarrow \mathrm{Neg}$ & $\mathrm{Neg} \rightarrow \mathrm{PI}$ & $\overline{~ W \rightarrow P I}$ \\
\hline & & 5.1 & 6.1 & 5.0 \\
\hline & & 4.6 & 5.3 & 1.5 \\
\hline & \multirow{4}{*}{ Behavioral } & $\mathrm{J} \rightarrow \mathrm{Alc}$ & Alc $\rightarrow$ PI & $\mathrm{J} \rightarrow \mathrm{PI}$ \\
\hline & & 4.3 & 1.1 & 9.2 \\
\hline & & $\mathrm{G} \rightarrow \mathrm{Alc}$ & $\mathrm{Alc} \rightarrow \mathrm{PI}$ & $\mathrm{G} \rightarrow \mathrm{PI}$ \\
\hline & & 7.0 & 0.4 & 0.7 \\
\hline \multirow{10}{*}{$\begin{array}{l}\text { White } \\
\text { vs } \\
\text { Black }\end{array}$} & \multirow{3}{*}{ Social } & $\mathrm{GI} \rightarrow \mathrm{Mar}$ & Mar $\rightarrow$ PI & $\mathrm{GI} \rightarrow \mathrm{PI}$ \\
\hline & & 13.8 & 2.8 & 5.4 \\
\hline & & 11.8 & 1.2 & 5.9 \\
\hline & \multirow{4}{*}{ Psychological } & $\mathrm{W} \rightarrow$ Som & Som $\rightarrow$ PI & W $\rightarrow$ PI \\
\hline & & 4.5 & 7.8 & 1.3 \\
\hline & & EdJ $\rightarrow$ Som & Som $\rightarrow$ PI & EdJ $\rightarrow$ PI \\
\hline & & 8.8 & 6.2 & 8.3 \\
\hline & \multirow{3}{*}{ Behavioral } & G $\rightarrow$ Alc & Alc $\rightarrow$ PI & 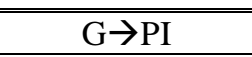 \\
\hline & & 3.1 & 1.6 & 0.7 \\
\hline & & 6.2 & 0.5 & 0.2 \\
\hline \multirow{11}{*}{$\begin{array}{c}\text { Black } \\
\text { vs } \\
\text { Hispanic }\end{array}$} & \multirow{4}{*}{ Social } & $\mathrm{GI} \rightarrow \mathrm{Mar}$ & Mar $\rightarrow$ PI & $\mathrm{GI} \rightarrow \mathrm{PI}$ \\
\hline & & 12.4 & 3.5 & 5.4 \\
\hline & & $\mathrm{I} \rightarrow \mathrm{Mar}$ & Mar $\rightarrow$ PI & $\mathrm{I} \rightarrow \mathrm{PI}$ \\
\hline & & 8.9 & 1.5 & 3.0 \\
\hline & \multirow{4}{*}{ Psychological } & $\mathrm{J} \rightarrow$ Som & Som $\rightarrow$ PI & $\mathrm{J} \rightarrow \mathrm{PI}$ \\
\hline & & 6.2 & 4.9 & 5.7 \\
\hline & & $\mathrm{W} \rightarrow \mathrm{Neg}$ & $\mathrm{Neg} \rightarrow \mathrm{PI}$ & $\mathrm{W} \rightarrow \mathrm{PI}$ \\
\hline & & 4.6 & 5.4 & 1.5 \\
\hline & \multirow{3}{*}{ Behavioral } & G $\rightarrow$ Alc & Alc $\rightarrow$ PI & G $\rightarrow$ PI \\
\hline & & 7.0 & 0.7 & 0.3 \\
\hline & & 7.0 & 0.5 & 0.7 \\
\hline
\end{tabular}

Note: D, $\{$ SPB $\}$, and PI were from Time 1

\section{(a) Whites versus Hispanics}

Social factors as intermediate variables

In both Whites and Hispanics, the social integration variable associated with SES/demographic variables was marital status (Table 21), Mar (i.e., D:GIMar:S), where the D variables gender $(\mathrm{G})$ and income (I) were jointly associated with marital status 
(Mar). Note that in this model and other similar models, there was no IV/DV distinction between $\mathrm{D}$ (demographic/SES) and $\mathrm{S}$ (social integration) variables because the objective of this step was to discover D variables that were associated with $\mathrm{S}$ (or $\mathrm{P}, \mathrm{B}$ ) variables. This step was followed by directed (specifying selected D variables as IVs and S variable as DV) modeling to compute uncertainty reduction in $\mathrm{S}$ variable and then compute uncertainty reduction in PI (physical impairment) as presented in Table $21\left(\beta^{\prime} \& \gamma^{\prime}\right.$ columns). Comparing path values for both groups, it was clear that gender and income were strongly related to marital status in Whites and Hispanics. The adjusted marital status to physical impairment path was low in both groups, however. Marital status appeared to be slightly more informative of physical impairment status in Hispanics than it was in Whites.

\section{Psychological factors as intermediate variables}

Wealth was moderately related to negative affect in Whites and Hispanics (Table 21). Overall, path values for the White group were slightly larger. The only path value prominently different across groups was the adjusted path from wealth to physical impairment, where wealth was more informative of physical impairment status in Whites controlling for negative affect (Neg). In Hispanics, after controlling for negative affect, the information gain about physical impairment due to wealth levels was very small $(1.5 \%)$. This indicates that wealth was strongly associated with physical impairment status in Whites but this relationship was weak in the Hispanic group. These moderately strong relationships between wealth and negative affect and, in turn, between negative affect and physical impairment status suggest possible indirect relationships between 
wealth and physical impairment in both Whites and Hispanics but mechanisms generating race/ethnic disparities are not prominent in these relationships given these variables.

Behavioral factors as intermediate variables

Common intermediate behavioral variable suggested by the neutral search was drinking (i.e., average level of alcoholic beverage consumption per week). Specific models for each group differed slightly, where the model for Whites was D:JAlc:B (Alc=drinking, $\mathrm{J}=$ job status) and for Hispanics it was D:GSmkAlc:B (Smk = smoking status; $\mathrm{G}=$ gender) but intermediate to physical impairment status path values were weak. Uncertainty reductions (i.e., values of $\alpha, \beta^{\prime} \& \gamma^{\prime}$ paths in Table 21) show that drinking (Alc) to physical impairment status path values were weak in both groups failing to suggest indirect relationships between SES and physical impairment status.

\section{(b) Whites versus Blacks}

Social factors as intermediate variables

In both Whites and Blacks, gender and income were jointly related to marital status (D:GIMar:S). Recall that in the White versus Hispanic group comparison, marital status was also the intermediate social variable selected, indicating the close relationship between marital status and SES/demographic variables in all three race/ethnic groups. Note that this was the same model examined in White versus Hispanic group comparison earlier. Path values are provided in Table 21. 


\section{Psychological factors as intermediate variables}

The neutral search for models in Whites suggested that wealth (W) was related to somatic symptoms (Som) given by the model D:WSom:P, whereas for Blacks, education (Ed) and employment status (J) were jointly associated with somatic symptoms (D:EdJSom:P), suggesting somatic symptoms (Som) as the intermediate variable related to SES/demographic variables in both groups. Because the associated SES variables were not identical, analyses focused on the effect of wealth in Whites and the joint effect of education and job status in Blacks. In Whites, the somatic symptoms variable was strongly related to physical impairment status, controlling for wealth. Moderate association of wealth with somatic symptoms and strong association of somatic symptoms with physical impairment status in the White group suggest potential indirect relationship between wealth and physical impairment status. In Blacks, employment status and education level jointly predicted somatic symptoms with considerable $(\% \Delta \mathrm{U}(\mathrm{Som})=8.8)$ percent uncertainty reduction in the somatic symptoms variable. The adjusted effect of somatic symptoms on physical impairment status, controlling for the joint effect of education and employment status was moderate. Thus, these SES variables were likely related to physical impairment status through somatic symptoms.

\section{Behavioral factors as intermediate variables}

Gender was associated with alcoholic beverage consumption (i.e., D:GAlc:B) in both Whites and Blacks. Note that earlier, in the corresponding White versus Hispanic comparison, alcoholic beverage consumption was also suggested as the intermediate 
variable. However, the weak uncertainty reductions in physical impairment due to drinking indicate lack of evidence for indirect associations in both groups.

\section{(c) Blacks versus Hispanics}

Social factors as intermediate variables

The neutral search suggested that marital status was associated with SES/demographic variables in Blacks and Hispanics with slight difference in complexity of the models. The model for Blacks (D:GIMar:S) had gender and income jointly related to marital status while the Hispanic group model (D:IMar:S) had only income associated with marital status but path values were weak (see Table 21).

\section{Psychological factors as intermediate variables}

Different psychological variables were related to different SES variables. In Blacks, somatic symptoms was related to employment status (D:JSom:P), and in Hispanics, negative affect was related to wealth (D:WNeg:P). Percent uncertainty reductions with different intermediate variables were computed.

The direct effect of employment status on somatic symptoms in Blacks was large and the effect of somatic symptoms on physical impairment status controlling for the direct effect of employment status was moderate. These moderate uncertainty reductions indicate possible indirect association between job status and physical impairment status through somatic symptoms in the Black group. In Hispanics, the effect of wealth on negative affect and the effect of negative affect on physical impairment, controlling for 
the direct effect of wealth, were moderate, suggesting potential indirect association of wealth with physical impairment through negative affect in this group.

\section{Behavioral factors as intermediate variables}

Two behavioral variables (D:GSmkAlc:B) in the Hispanic group were jointly related to gender while one behavioral variable (D:GAlc:B) was related to gender in Blacks. Drinking (Alc) was used as intermediate variable in comparing Blacks and Hispanics. In all the analyses, indirect path values were weak (Table 21).

\section{Summary}

In this section, there was a clear pattern that was similar in all race/ethnic groups with few exceptions. First, results indicated that SES/demographic variable(s) strongly predicted the intermediate variable whether that intermediate variable was psychological, social or behavioral variable. The second observation that runs across groups was that the effect of psychological variables on physical impairment, controlling for the effect of the SES/demographic variable(s) was stronger than the effects of social integration or behavioral variables. Third, the behavioral factors to physical impairment links were almost zero in all race/ethnic groups, indicating lack of evidence for indirect relationships. This means that race/ethnic differences in health status, to a large extent, may not be attributed to variation in behavioral factors. Finally, while education level, job status and income were strongly related to physical impairment status, gender and wealth were weakly linked to physical impairment status, controlling for the intermediate variables. 


\subsection{Longitudinal Analysis of Physical Impairment Status}

The objective of this section was similar to the objective in section 6.1 but using data from the second time point for social $(\mathrm{S})$, psychological $(\mathrm{P})$, and behavioral factors (B) to predict physical impairment status (PI) at the third time point. Demographic/SES (D) data were all from the first time point.

The research questions in this section were:

RQ3a: How do SES/demographic variables at the first time point interact with social integration variables, psychological variables, and behavioral variables at the second time point, to predict physical impairment status at the third time point?

RQ3b: Do such predictions differ by race/ethnic groups?

Table 22 provides results of models with loops comparing race/ethnic groups.

Overall, models were less complex relative to the cross-sectional models (compare Table 14). (Note: in White vs Hispanic, upper model is for Whites and lower model for Hispanics).

Table 22: Summary of inter-race/ethnic comparison of longitudinal models

\begin{tabular}{|c|l|}
\hline Race/ethnicity & \multicolumn{1}{|c|}{ Model } \\
\hline $\begin{array}{c}\text { White } \\
\text { vs } \\
\text { Hispanic }\end{array}$ & BmiPI : ExercisePI : VolunteerPI \\
\cline { 2 - 2 } $\begin{array}{c}\text { White } \\
\text { vs } \\
\text { Black }\end{array}$ & SomaticPI : NegativePI \\
\cline { 2 - 2 } & SomaticPI : NegativePI : JobPI : VolunteerI \\
\hline \hline $\begin{array}{c}\text { Black } \\
\text { vs } \\
\text { Hispanic }\end{array}$ & SomaticPI : NegativePI : BmiPI : VolunteerPI \\
\cline { 2 - 2 } & SomaticPI : NegativePI \\
\hline
\end{tabular}

Note that in the analyses below, abbreviations of the variable names are used as follows: Job status=J, Somatic symptoms=Som, Exercise=Ex; Volunteering=Vo; Bmi=Bmi; Positive affect=Pos; Negative affect=Neg

Positive affect was reverse coded. Bmi=body mass index; PI=physical impairment 


\section{(a) Whites versus Hispanics}

Models with loops suggested different predictive variables in Whites and Hispanics (Table 23), that is, there were no overlapping predictors. Unlike models without loops (bolded variables appeared in models without loops), models with loops were slightly more complex and percent uncertainty reduction in physical impairment in models with loops for both groups was substantial. Note that there were no interaction effects in any of the models and all predictive variables were either behavioral (Bmi \& Ex), social (Vo) and/or psychological factors (Som \& Neg) which were measured two years prior to the outcome variable.

Table 23: Models with loops for White versus Hispanic groups

\begin{tabular}{|l|l|l|l|}
\hline Race/ethnicity & \multicolumn{1}{|c|}{ Model } & $\Delta \mathrm{df}$ & $\% \Delta \mathrm{U}(\mathrm{PI})$ \\
\hline White & ExPI:BmiPI:VoPI & 5 & 10.9 \\
\hline Hispanic & SomPI:NegPI & 2 & 9.7 \\
\hline
\end{tabular}

Comparative analysis of composite IV states was not possible due to non-overlap of predictive variables in the models. However, further analysis of each model on its own was conducted and results of model fit for the White group (Table 20) and for the Hispanic group (Table 21) are provided below. 
Table 24: Fit results for BmiPI:ExPI:VoPI for the White group

\begin{tabular}{|c|c|c|c|c|c|c|c|c|}
\hline \multirow[t]{2}{*}{ Race/ethnicity } & \multicolumn{3}{|c|}{ IV } & \multirow{2}{*}{$\begin{array}{l}\text { Data } \\
\text { freq }\end{array}$} & \multicolumn{4}{|c|}{$\begin{array}{l}\text { Model } \\
\text { calc. } \mathrm{q}(\mathrm{DV} \mid \mathrm{IV})\end{array}$} \\
\hline & Bmi & Ex & Vo & & $\mathrm{PI}=0$ & $\mathrm{PI}=1$ & $\mathrm{p}$ (margin) & Ratio, $\mathrm{PI}=1$ \\
\hline \multirow{13}{*}{ White } & 1 & 0 & 0 & 65 & 68.8 & 31.2 & 0.297 & 1.22 \\
\hline & 1 & 0 & 1 & 13 & 87.3 & 12.7 & 0.310 & 0.50 \\
\hline & 1 & 1 & 0 & 13 & 94.1 & 5.9 & 0.119 & 0.23 \\
\hline & 1 & 1 & 1 & 8 & 98.0 & 2.0 & 0.127 & 0.08 \\
\hline & 1 & 2 & 0 & 19 & 90.4 & 9.6 & 0.121 & 0.37 \\
\hline & 1 & 2 & 1 & 11 & 96.7 & 3.3 & 0.108 & 0.13 \\
\hline & $\beta_{2}$ & 0 & 0 & 205 & 61.3 & 38.7 & 0.000 & 1.52 \\
\hline & 2 & 0 & 1 & 52 & 83.1 & 16.9 & 0.159 & 0.66 \\
\hline & 2 & 1 & 0 & 23 & 92.0 & 8.0 & 0.060 & 0.31 \\
\hline & 2 & 1 & 1 & 9 & 97.3 & 2.7 & 0.140 & 0.11 \\
\hline & 2 & 2 & 0 & 39 & 87.2 & 12.8 & 0.074 & 0.50 \\
\hline & $\alpha_{2}$ & 2 & 1 & 18 & 95.5 & 4.5 & 0.047 & 0.18 \\
\hline & \multicolumn{4}{|c|}{ Marginal probability } & 74.5 & 25.5 & & \\
\hline
\end{tabular}

Note: ${ }^{\alpha}=$ protective $\&^{\beta}=$ risky states

In the White group (Table 24), confining analysis of states only to the risky or protective ones (rows in red or ${ }^{\beta}$ row $\&$ blue or ${ }^{\alpha}$ row respectively), relative to marginal risk, indicated that there were only two composite IV states with such qualities. Overweight/obese $(\mathrm{Bmi}=2)$ Whites who engaged in vigorous physical activity $(\mathrm{Ex}=2)$ and volunteered in organizations $\left(\mathrm{V}_{\mathrm{o}}=1\right)$ had substantially lower risk of physical impairment. Regarding the risky predictor states, risk of physical impairment in overweight/obese Whites, who did not engage in any physical activity $(\mathrm{Ex}=0)$, and did not volunteer in organizations $(\mathrm{Vo}=0)$, increased by over $50 \%$ relative to the marginal risk posed to the sample.

Predictive variables in the Hispanic model (Table 23) were all psychological variables involving two of the three sub-scales of the psychological factors used in this study. Fit results (Table 25) showed that concurrent absence of somatic symptoms and negative affect (i.e., Som $=0 \& \mathrm{Neg}=0$ ), were related to decreased risk of physical 
impairment. Conversely, simultaneous presence of somatic symptoms and negative affect (Som=1 \& Neg=1) were related to over $50 \%$ increase in risk of physical impairment relative to the marginal risk to the group.

Table 25: Fit results for SomPI:NegPI for the Hispanic group

\begin{tabular}{|c|c|c|c|c|c|c|c|}
\hline \multirow[t]{2}{*}{ Race/ethnicity } & \multicolumn{2}{|c|}{ IV } & \multirow{2}{*}{$\begin{array}{l}\text { Data } \\
\text { freq }\end{array}$} & \multicolumn{2}{|c|}{$\begin{array}{c}\text { Model } \\
\text { calc. } \mathrm{q}(\mathrm{DV} \mid \mathrm{IV})\end{array}$} & \multirow[b]{2}{*}{$\mathrm{p}($ margin $)$} & \multirow[b]{2}{*}{ Ratio, PI=1 } \\
\hline & Som & Neg & & $\mathrm{PI}=0$ & $\mathrm{PI}=1$ & & \\
\hline \multirow{5}{*}{ Hispanic } & ${ }^{\alpha} 0$ & 0 & 167 & 85.5 & 14.5 & 0.011 & 0.64 \\
\hline & 0 & 1 & 33 & 76.5 & 23.5 & 0.924 & 1.03 \\
\hline & 1 & 0 & 106 & 76.6 & 23.4 & 0.891 & 1.02 \\
\hline & $\beta_{1}$ & 1 & 102 & 64.5 & 35.5 & 0.002 & 1.56 \\
\hline & \multicolumn{3}{|c|}{ Marginal prob. } & 77.2 & 22.8 & & \\
\hline
\end{tabular}

The presence of one psychological variable with the absence of the second (i.e., $\mathrm{Som}=0 \& \mathrm{Neg}=1$, or $\mathrm{Som}=1 \& \mathrm{Neg}=0$ ), did not appear to increase risk compared to the marginal risk to the sample although previous cross-sectional results showed presence of somatic symptoms alone increased risk of physical impairment.

\section{Summary}

All predictors of physical impairment were unique in each race/ethnic group. Body mass index, consistent with earlier findings, seemed unimportant as seen in the protective and risky composite IV states for the White group. Rather, engaging in physical activity and volunteering in organizations seemed to predict physical impairment status. In the Hispanic group, results of risky and protective composite IV states involved concurrent presence of the two psychological variables. Presence of somatic symptoms alone tended to increase risk of physical impairment in the cross-sectional models but this was not the case in the longitudinal models above. 


\section{(b) Whites versus Blacks}

Percent uncertainty reduction in models with loops was large although complexity did not substantially increase (Table 26). Two variables, somatic symptoms and volunteering in organizations predicted physical impairment in both groups. But, physical activity (Ex) was unique to the model for Whites, whereas, job status (J) and negative affect (Neg) were unique to the model for Blacks (in models without loops only bolded variables appeared).

Table 26: Models for White and Black groups

\begin{tabular}{|l|l|l|l|}
\hline Race/ethnicity & \multicolumn{1}{|c|}{ Model } & $\Delta \mathrm{df}$ & $\% \Delta \mathrm{U}(\mathrm{PI})$ \\
\hline White & SomPI:VoPI:ExPI & 4 & 9 \\
\hline Black & SomPI:VoPI:NegPI:JPI & 5 & 15.5 \\
\hline
\end{tabular}

In order to explore variation in risk due to composite IV states, a model with only the overlaps (SomPI:VoPI) was fit in both race/ethnic groups (Table 27). A notable result involved absence of somatic symptoms $(\mathrm{Som}=0)$ and absence of volunteering in organizations $\left(\mathrm{Vo}_{\mathrm{o}}=0\right)$. In Whites, concurrent absence of somatic symptoms and volunteering posed similar risk of physical impairment to the marginal risk for the total sample, whereas, in Blacks, absence of somatic symptoms and absence of volunteering significantly decreased risk of physical impairment (row in green or ${ }^{\lambda}$ row). 
Table 27: Fit results for SomPI:VoPI for White and Black groups

\begin{tabular}{|c|c|c|c|c|c|c|c|}
\hline \multirow[t]{2}{*}{ Race/ethnicity } & \multicolumn{2}{|c|}{ IV } & \multirow{2}{*}{$\begin{array}{l}\text { Data } \\
\text { freq }\end{array}$} & \multicolumn{2}{|c|}{$\begin{array}{c}\text { Model } \\
\text { calc. } \mathrm{q}(\mathrm{DV} \mid \mathrm{IV})\end{array}$} & \multirow[b]{2}{*}{$\mathrm{p}($ margin $)$} & \multirow[b]{2}{*}{ Ratio, $\mathrm{PI}=1$} \\
\hline & Som & Vo & & $\mathrm{PI}=0$ & $\mathrm{PI}=1$ & & \\
\hline \multirow{5}{*}{ White } & 0 & 0 & 220 & 83.6 & 16.4 & 0.142 & 0.80 \\
\hline & ${ }^{\alpha} 0$ & 1 & 134 & 92.7 & 7.3 & 0.000 & 0.36 \\
\hline & $\beta_{1} 1$ & 0 & 256 & 68.0 & 32.0 & 0.000 & 1.56 \\
\hline & 1 & 1 & 70 & 84.1 & 15.9 & 0.353 & 0.78 \\
\hline & \multicolumn{3}{|c|}{ Marginal prob. } & 79.6 & 20.4 & & \\
\hline \multirow{5}{*}{ Black } & $\lambda 0$ & 0 & 154 & 88.6 & 11.4 & 0.010 & 0.58 \\
\hline & ${ }^{\alpha} 0$ & 1 & 134 & 95.2 & 4.8 & 0.000 & 0.24 \\
\hline & $\begin{array}{ll}\beta & 1 \\
\end{array}$ & 0 & 276 & 66.9 & 33.1 & 0.000 & 1.68 \\
\hline & 1 & 1 & 115 & 83.8 & 16.2 & 0.338 & 0.82 \\
\hline & \multicolumn{3}{|c|}{ Marginal prob. } & 80.3 & 19.7 & & \\
\hline
\end{tabular}

Note: ${ }^{\alpha}=$ protective $\&^{\beta}=$ risky states in both groups; ${ }^{\lambda}=$ unique protective states in Blacks

Common protective states for Whites and Blacks were absence of somatic symptoms $(\mathrm{Som}=0)$ and volunteering in organizations $(\mathrm{Vo}=1)$, where, the risk was significantly lower than the respective marginal risk to each group (rows in blue or ${ }^{\alpha}$ row). Conversely, risk of physical impairment for individuals with somatic symptoms who did not volunteer in organizations was similar in both groups and significantly higher than the marginal risk to the sample in each group (row $s$ in red or ${ }^{\beta}$ row).

\section{Summary}

Whites and Blacks who volunteered and had no somatic symptoms were better off compared to individuals with somatic symptoms and did not volunteer in charitable organizations. The risk to individuals who volunteered and had somatic symptoms was not different from the marginal risk to the group in both Whites and Blacks. Blacks who did not volunteer and had no somatic symptoms fared better than their White counterparts, however. This difference, conceivably, points to the utility of volunteering in organizations to Whites compared to its utility for Blacks. 


\section{(c) Blacks versus Hispanics}

The model for the Hispanic group (Table 28) was identical to that obtained in comparing Whites versus Hispanics (Table 23). In Blacks, at least one from each type of intermediate variables (mediators) independently predicted physical impairment with substantial percent uncertainty reduction. Both psychological variables that appeared in the model for Hispanics also appeared in the model for Blacks.

Table 28: Models for Black and Hispanic group

\begin{tabular}{|l|l|l|l|}
\hline Race/ethnicity & \multicolumn{1}{|c|}{ Model } & $\Delta \mathrm{df}$ & $\% \Delta \mathrm{U}(\mathrm{PI})$ \\
\hline Black & SomPI:NegPI:BmiPI:VoPI & 5 & 15.4 \\
\hline Hispanic & SomPI:NegPI & 2 & 9.7 \\
\hline
\end{tabular}

A model only with the overlapping variables (SomPI:NegPI) was fit to examine risks associated with various composite IV states in Blacks and Hispanics as provided below (Table 29) and differences by groups were examined.

This was one of the interesting results not because similar predictor states posed significantly more or less risk, but because the risk with the concurrent presence of somatic and negative affect $(\mathrm{Som}=1 \& \mathrm{Neg}=1)$ were nearly identical in both groups as was the protective effects in the concurrent absence of both symptoms (Som=0 \& $\mathrm{Neg}=0$ ). In Blacks and Hispanics with somatic symptoms and negative affect, risks of physical impairment increased by $79 \%$ and $73 \%$, respectively, compared to the respective marginal risk. The risk of having one and not the other psychological variable (i.e., Som $=1 \& \mathrm{Neg}=0$, or Som=0 \& Neg=1) was not significantly different from the marginal risk. These results point to absence of inter-group differences in physical limitations 
given these overlapping variables, thus failing to indicate race/ethnicity based differences in health outcomes but suggest intra-group differences.

Table 29: Fit results for SomPI:NegPI for Black and Hispanic group

\begin{tabular}{|c|c|c|c|c|c|c|c|}
\hline \multirow{3}{*}{ Race/ethnicity } & \multicolumn{2}{|c|}{ IV } & Data & \multicolumn{2}{c|}{$\begin{array}{c}\text { Model } \\
\text { calc. q(DV|IV) }\end{array}$} \\
\cline { 2 - 8 } & Som & Neg & freq & PI=0 & PI=1 & p(margin) & Ratio, PI=1 \\
\hline \multirow{5}{*}{ Black } & ${ }^{\alpha} 0$ & 0 & 122 & 92.6 & 7.4 & 0.000 & 0.33 \\
\cline { 2 - 8 } & 0 & 1 & 19 & 84.3 & 15.7 & 0.490 & 0.70 \\
\cline { 2 - 8 } & 1 & 0 & 124 & 77.4 & 22.6 & 0.990 & 1.00 \\
\cline { 2 - 8 } & $\beta 1$ & 1 & 111 & 59.4 & 40.6 & 0.000 & 1.79 \\
\cline { 2 - 8 } & \multicolumn{3}{|c|}{ Marginal probability } & 77.4 & 22.6 & & \\
\hline \multirow{5}{*}{ Hispanic } & ${ }^{\alpha} 0$ & 0 & 142 & 91.0 & 9.0 & 0.000 & 0.39 \\
\cline { 2 - 8 } & 0 & 1 & 34 & 81.9 & 18.1 & 0.471 & 0.77 \\
\cline { 2 - 8 } & 1 & 0 & 65 & 76.7 & 23.3 & 0.992 & 1.00 \\
\cline { 2 - 8 } & ${ }^{\beta} 1$ & 1 & 131 & 59.7 & 40.3 & 0.000 & 1.73 \\
\cline { 2 - 7 } & \multicolumn{2}{|c|}{ Marginal probability } & 76.6 & 23.4 & & \\
\hline
\end{tabular}

Note: ${ }^{\alpha}=$ protective $\&^{\beta}=$ risky states in both groups

At this point, it would be reasonable to speculate on the reason for similarity of these models. In preparing the datasets, the sample size for Whites was large, and in matching White cases with Hispanics, and Black cases with Hispanics, nearly all Hispanic cases were retained because there were sufficient White and Black cases resembling the Hispanic sample in the covariates (D/SES) at the first time point. Naturally, then, the models for Hispanics versus White comparison, and Hispanic versus Black comparison would either be similar or identical as observed.

\section{Summary}

Hispanics and Blacks with somatic symptoms and negative affect were worse off compared to their counterparts without these depressive symptoms. Further, level of risk in the concurrent presence of these specific states was similar in both race/ethnic groups. Presence of only one symptom and absence of the other (e.g., Som=1 or Neg=0) did not 
pose sufficiently different risk compared to the marginal risk in each group. Given these overlapping variables, composite IV state analysis showed intra-group differences but failed to support inter-group differences.

\subsection{Longitudinal Path Analysis of Physical Impairment Status}

This section provides results for relationships between SES/demographic variables and intermediate variables, as well as relationships between the intermediate variables and the outcome variable. The procedure repeats the steps for section 6.2 but using social, psychological and behavioral variables from the second time point to predict physical impairment status at the third time point.

The research questions in this section were:

RQ4a: Do relationships between SES/demographic variables at the first time point and intermediate variables at the second time point differ by race/ethnicity?

RQ4b: Do relationships between intermediate variables at the second time point and physical impairment at the third time point differ by race/ethnicity?

The difference between Figure 23 and Figure 24 is that the S, P, and B variables were from the second time point and physical impairment (PI) was from the third time point in Figure 24 as indicated by the subscripts. The subscript $i$ indexes D, S, P, and B variables that appear in the selected models and subscript $t$ is time indicator. Corresponding values for path values $\left(\alpha, \beta^{\prime} \& \gamma^{\prime}\right)$ and the selected variables are given in Table 30. 


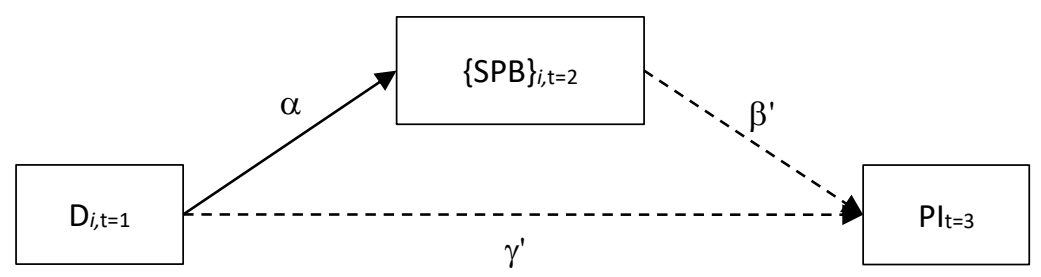

Figure 24: Longitudinal path modeling of physical impairment status Note: $\mathrm{i}=$ selected variable(s) \& $\mathrm{t}=$ time

Longitudinal path analysis results (Table 30) for the physical impairment outcome variable differ from the cross-sectional path analysis results provided earlier (see Table 21). In the cross-sectional analysis, there were eight intermediate variables $\{\mathbf{S P B}\}$ that were at least weakly (i.e., $\geq 3.5 \%$ uncertainty reduction) related to physical impairment at the third time point, while in the longitudinal analysis here, there were only five such path values (see $\beta^{\prime}$ column in Table 30), indicating stronger cross-sectional relationships in this dataset. 
Table 30: Uncertainty reductions for longitudinal path models of physical impairment status

\begin{tabular}{|c|c|c|c|c|}
\hline Race/ethnicity & $\{$ SPB $\}$ factors & $\boldsymbol{\alpha}=\mathrm{T}(\mathrm{D}:\{\mathrm{SPB}\})$ & $\boldsymbol{\beta}^{\prime}=\mathrm{T}_{\mathrm{D}}(\{\mathrm{SPB}\}: \mathrm{PI})$ & $\gamma^{\prime}=\mathrm{T}_{\{\mathrm{SPB}\}}(\mathrm{D}: \mathrm{PI})$ \\
\hline \multirow{12}{*}{$\begin{array}{l}\text { White } \\
\text { vs } \\
\text { Hispanic }\end{array}$} & \multirow{4}{*}{ Social } & $\mathrm{GI} \rightarrow \mathrm{Mar}$ & $\mathrm{Mar} \rightarrow \mathrm{PI}$ & $\mathrm{GI} \rightarrow \mathrm{PI}$ \\
\hline & & 15.2 & 4.0 & 4.7 \\
\hline & & $\mathrm{I} \rightarrow \mathrm{Mar}$ & Mar $\rightarrow$ PI & $\mathrm{I} \rightarrow \mathrm{PI}$ \\
\hline & & 8.5 & 1.8 & 2.3 \\
\hline & \multirow{4}{*}{ Psychological } & $\mathrm{J} \rightarrow \mathrm{Neg}$ & Som $\rightarrow$ PI & $\mathrm{J} \rightarrow \mathrm{PI}$ \\
\hline & & 4.3 & 1.6 & 1.8 \\
\hline & & $\mathrm{J} \rightarrow$ Pos & Pos $\rightarrow$ PI & $\mathrm{J} \rightarrow \mathrm{PI}$ \\
\hline & & 4.6 & 2.1 & 1.8 \\
\hline & \multirow{4}{*}{ Behavioral } & $\mathrm{J} \rightarrow$ Alc & Alc $\rightarrow$ PI & $\mathrm{J} \rightarrow \mathrm{PI}$ \\
\hline & & 6.4 & 2.1 & 3.5 \\
\hline & & $\mathrm{G} \rightarrow \mathrm{Alc}$ & Alc $\rightarrow$ PI & $\mathrm{G} \rightarrow \mathrm{PI}$ \\
\hline & & 8.6 & 0.6 & 0.4 \\
\hline \multirow{11}{*}{$\begin{array}{l}\text { White } \\
\text { vs } \\
\text { Black }\end{array}$} & \multirow{3}{*}{ Social } & $\mathrm{GI} \rightarrow \mathrm{Mar}$ & Mar $\rightarrow$ PI & $\mathrm{GI} \rightarrow \mathrm{PI}$ \\
\hline & & 14.6 & 4.3 & 4.7 \\
\hline & & 12.2 & 1.9 & 4.6 \\
\hline & \multirow{4}{*}{ Psychological } & GEd $\rightarrow \mathrm{Neg}$ & $\mathrm{Neg} \rightarrow \mathrm{PI}$ & Ged $\rightarrow$ PI \\
\hline & & 7.2 & 3.1 & 2.9 \\
\hline & & $\mathrm{Ed} \rightarrow$ Som & Som $\rightarrow$ PI & $\mathrm{Ed} \rightarrow \mathrm{PI}$ \\
\hline & & 5.9 & 4.1 & 0.5 \\
\hline & \multirow{4}{*}{ Behavioral } & $\mathrm{Ed} \rightarrow$ Alc & Alc $\rightarrow$ PI & $\mathrm{Ed} \rightarrow \mathrm{PI}$ \\
\hline & & 3.8 & 1.0 & 2.0 \\
\hline & & $\mathrm{G} \rightarrow$ Alc & $\mathrm{Alc} \rightarrow \mathrm{PI}$ & $\mathrm{G} \rightarrow \mathrm{PI}$ \\
\hline & & 4.7 & 0.5 & 0.2 \\
\hline \multirow{11}{*}{$\begin{array}{c}\text { Black } \\
\text { vs } \\
\text { Hispanic }\end{array}$} & \multirow{4}{*}{ Social } & GI $\rightarrow$ Mar & Mar $\rightarrow$ PI & $\mathrm{GI} \rightarrow \mathrm{PI}$ \\
\hline & & 13.0 & 4.4 & 7.0 \\
\hline & & $\mathrm{I} \rightarrow \mathrm{Mar}$ & Mar $\rightarrow$ PI & $\mathrm{I} \rightarrow \mathrm{PI}$ \\
\hline & & 8.5 & 1.8 & 2.3 \\
\hline & \multirow{4}{*}{ Psychological } & Ed $\rightarrow$ Som & Som $\rightarrow$ PI & $\mathrm{J} \rightarrow \mathrm{PI}$ \\
\hline & & 5.7 & 5.5 & 0.9 \\
\hline & & $\mathrm{J} \rightarrow$ Pos & Pos $\rightarrow$ PI & $\mathrm{W} \rightarrow \mathrm{PI}$ \\
\hline & & 4.6 & 2.1 & 2.0 \\
\hline & \multirow{3}{*}{ Behavioral } & $\mathrm{G} \rightarrow \mathrm{Alc}$ & $\mathrm{Alc} \rightarrow \mathrm{PI}$ & $\mathrm{G} \rightarrow \mathrm{PI}$ \\
\hline & & 6.0 & 0.7 & 0.2 \\
\hline & & 8.6 & 0.6 & 0.4 \\
\hline
\end{tabular}

Note: D variables were from Time 1, $\{\mathrm{SPB}\}$ variables from Time 2 and PI from Time 3

\section{(a) Whites versus Hispanics}

\section{Social factors as intermediate variables}

Gender and income were jointly associated with marital status at the second time point (D:GIMar:S) in the White group while only income was related to marital status (D:IMar:S) in the Hispanic group. The joint effect of gender and income on marital status 
at the second time point was substantial $(\% \Delta \mathrm{U}(\mathrm{Mar})=15.2 \%)$. Percent uncertainty reduction in physical impairment controlling for the joint effect of gender and income was moderate, indicating potential indirect relationship between these SES variables and physical impairment status. The paths were weak in models for the Hispanic group (see Table 30 for results).

Psychological factors as intermediate variables

The neutral search in the White group suggested that job status was associated with somatic symptoms at the second time point (D:JSom:P), whereas in the Hispanic group, job status was associated with positive affect (D:JPos:P) at the second time point. Because the selected intermediate variables were different, direct and indirect path values were provided for each race/ethnic group and indirect effects were not strong.

\section{Behavioral factors as intermediate variables}

In the White group, the neutral search suggested that drinking was associated with job status (D:JAlc:B), while, in the Hispanic group, drinking and smoking were jointly associated with gender (D:GAlcSmk:B). Separate directed system analysis was conducted for Whites and Hispanics with drinking (Alc) as the intermediate variable (Table 30).

\section{(b) Whites versus Blacks}

\section{Social factors as intermediate variables}

The neutral search to identify associations between SES/demographic and social variables in Whites and Blacks suggested that gender and income were jointly associated 
with marital status (D:GIMar:S) in both groups. Percent uncertainty reductions in marital status due to the joint effect of gender and income were large. The effect of marital status on physical impairment, controlling for the direct joint effect of gender and income in the White group was moderate (4.3\%), suggesting possible indirect relationship between SES/demographic variables and physical impairment status. The effect of marital status on physical impairment status, controlling for the direct joint effect of gender and income was weak failing to support the hypothesized indirect relationship in the Black group.

\section{Psychological factors as intermediate variables}

In the White versus Black comparison, the best model from neutral search for the White group was, D:GEdNeg:P, where gender $(\mathrm{G})$ and education $(\mathrm{Ed})$ were jointly associated with negative affect (Neg) at the second time point. For the Black group, the model from neutral search indicated that only education was associated with somatic symptoms at the second time point (D:EdSom:P).

The effect of negative affect on physical impairment status, controlling for the joint effect of gender and education was weak in Whites. In the Black group, uncertainty reduction in somatic symptoms due to education level was moderate and the effect of somatic symptoms on physical impairment, controlling for the direct effect of education was also moderate, suggesting possible indirect association between education and physical impairment through somatic symptoms. 


\section{Behavioral factors as intermediate variables}

The behavioral variable associated with SES/demographic variables in both Whites and Blacks was drinking. However, only gender (D:GAlc:B) in Blacks and education level (D:EdAlc:B) in Whites was associated with drinking. Behavioral to physical health status paths were weak in both groups as indicated in Table 30 .

\section{(c) Blacks versus Hispanics}

\section{Social factors as intermediate variables}

Gender and income were jointly related to marital status at the second time point in the Black group (D:GIMar:S), whereas, only income was related to marital status in the Hispanic group (D:IMar:S). In the Black group, percent uncertainty reduction in marital status due to the joint effect of gender and income was large (13\%). Further, the effect of marital status on physical impairment, controlling for the direct joint effect of income and gender on physical impairment was moderate (4.4\%). These path values suggest potential indirect association between the SES/demographic variables and physical impairment status in the Black group. However, the indirect path values were weak in the Hispanic group.

\section{Psychological factors as intermediate variables}

The model from neutral search for the Black group was D:EdSom:P, where education was associated with somatic symptoms at the second time point. In the Hispanic group the neutral model suggested that positive affect was related to employment status (D:JPos:P). Once again, the psychological variables that were 
associated with SES/demographic variables for the two races differed and separate analyses were conducted (Table 30).

In the Black group, the effect of education on somatic symptoms $(5.7 \%)$ and the effect of somatic symptoms on physical impairment status (5.5\%), controlling for the direct effect of education on physical impairment status was moderate. These moderate path values suggest potential indirect relationships between education level and physical impairment status in the Black group. Recall that employment status was also moderately related to somatic symptoms and, in turn, the somatic symptoms variable was moderately related to physical impairment status in the Black group for the cross-sectional path models comparing Blacks and Hispanics (Table 21). In the Hispanic group, path values were weak.

\section{Behavioral factors as intermediate variables}

In the Black group, drinking was associated with gender (D:GAlc:B), while in Hispanics, drinking and smoking were jointly associated with gender (D:GAlcSmk:B). Path values in the Black and Hispanic groups were weak, thus, indirect relationships, given these variables, were not plausible, in both groups.

\section{Summary}

Patterns of longitudinal relationships were similar to the cross-sectional relationships. Several relationships were either identical or similar to the relationships in cross-sectional models. However, the strength of longitudinal relationships was weaker compared to the cross-sectional relationships. Overall, bivariate relationships, that is, the 
direct relationships, were stronger when the intermediate variable was a psychological variable in the Black group and marital status in the White group. As in the crosssectional models, marital status was the only social variable selected in all race/ethnic groups, and drinking was the main behavioral variable associated with SES/demographic variables in all race/ethnic groups. Few indirect relationships in Whites and Blacks appeared to be moderately strong.

\section{Overnight Hospitalization}

This section provides cross-sectional results for the overnight hospitalization outcome variable $(0 / 1=$ not hospitalized/hospitalized). The steps in Section 6.1-6.4 were used with overnight hospitalization (HO) as the outcome variable instead of physical impairment status (PI).

\subsection{Cross-Sectional Analysis of Overnight Hospitalization}

This section explores association of SES, social, psychological, and behavioral variables with overnight hospitalization using cross-sectional data.

The research questions in this section were:

RQ5a: How do SES/demographic variables, social variables, psychological variables, and behavioral variables interact in predicting overnight hospitalization?

RQ5b: Do such predictions differ by race/ethnic groups?

Overall, summary of cross-sectional results indicated that, in the White group, hospitalization was not related to SES/demographic, social, psychological or behavioral 
variables. The models for Hispanics indicated that gender and negative affect independently predicted overnight hospitalization. Comparing Blacks with Hispanics, job status was related to overnight hospitalization in Blacks while the model for the Hispanic group was identical to the model suggested in White versus Hispanic comparison (Table $31)$.

Table 31: Racial and ethnic comparison of cross-sectional models

\begin{tabular}{|c|l|}
\hline Race/ethnicity & Model \\
\hline $\begin{array}{c}\text { White } \\
\text { vs } \\
\text { Hispanics }\end{array}$ & -- \\
\cline { 2 - 2 } $\begin{array}{c}\text { White } \\
\text { vs } \\
\text { Blacks }\end{array}$ & GHo:NegHo \\
\hline $\begin{array}{c}\text { Blacks } \\
\text { vs } \\
\text { Hispanics }\end{array}$ & -- \\
\hline \multirow{2}{*}{} & JHo \\
\cline { 2 - 2 } & GHo:NegHo \\
\hline
\end{tabular}

\section{(a) Whites versus Hispanics}

In Whites, none of the SES/demographic, social, psychological, and behavioral factors was related to overnight hospitalization status indicating that even the more refined approach, model search with loops allowed, failed to select any predictive variables. But, gender and negative affect were independently associated with overnight hospitalization status in the Hispanic group (Table 32).

Table 32: Models with loops for White and Hispanic groups

\begin{tabular}{|l|l|r|r|}
\hline Race/ethnicity & \multicolumn{1}{|c|}{ Model } & $\Delta \mathrm{df}$ & $\% \Delta \mathrm{U}(\mathrm{Ho})$ \\
\hline White & IV:Ho & 0 & 0 \\
\hline Hispanic & GHo:NegHo & 2 & 4.3 \\
\hline
\end{tabular}


To examine composite predictor states that increased or decreased risk of physical impairment, the model for Hispanics (GHo:NegHo) was fit. Being male $(\mathrm{G}=0)$ and reporting negative affect raised the risk for overnight hospitalization to almost double the marginal risk to the sample. However, overnight hospitalization for females reporting negative affect was not significantly different from the marginal risk to the group.

Females without negative affect fared better than males without negative affect, indicating differences by gender within the Hispanic group. It appears, then, that gender influences overnight hospitalization status and being female is protective in this group.

Table 33: Fit results for GHo:NegHo for the Hispanic group

\begin{tabular}{|c|c|c|c|c|c|c|}
\hline \multicolumn{2}{|c|}{ IV } & \multirow{2}{*}{$\begin{array}{l}\text { Data } \\
\text { freq }\end{array}$} & \multicolumn{4}{|c|}{$\begin{array}{l}\text { Model } \\
\text { calc. q(DV|IV) }\end{array}$} \\
\hline $\mathrm{G}$ & $\mathrm{Neg}$ & & $\mathrm{Ho}=0$ & $\mathrm{Ho}=1$ & $\mathrm{p}($ margin $)$ & Ratio, $\mathrm{Ho}=1$ \\
\hline 0 & 0 & 123 & 81.7 & 18.3 & 0.903 & 1.02 \\
\hline${ }^{\beta} 0$ & 1 & 60 & 65.9 & 34.1 & 0.001 & 1.91 \\
\hline${ }^{\alpha} 1$ & 0 & 142 & 90.5 & 9.5 & 0.009 & 0.53 \\
\hline 1 & 1 & 100 & 80.5 & 19.5 & 0.67 & 1.09 \\
\hline & & & 82.1 & 17.9 & & \\
\hline
\end{tabular}

Note: ${ }^{\alpha}=$ protective $\&^{\beta}=$ risky states

\section{(b) Whites versus Blacks}

Like the models without loops, the more refined approach of modeling with loops also failed to suggest predictive variables in Whites and Blacks. The best model suggested was the independence model (IV:Ho) in both Whites and Blacks.

\section{(c) Blacks versus Hispanics}

Again, the Hispanic model with loops was identical to an earlier model (Table 32) comparing Whites and Hispanics. The predictive variable in Blacks was job status. 
Percent uncertainty reductions in both the Black and Hispanic models were small (Table 34).

Table 34: Models with loops for Black and Hispanic groups

\begin{tabular}{|l|l|r|r|}
\hline Race/ethnicity & \multicolumn{1}{|c|}{ Model } & \multicolumn{1}{c|}{$\Delta$ df } & \multicolumn{1}{l|}{$\% \Delta \mathrm{U}(\mathrm{Ho})$} \\
\hline Black & JHo & 2 & 3.9 \\
\hline Hispanic & GHo:NegHo & 2 & 4.3 \\
\hline
\end{tabular}

Because there were no common predictor variables in the above models analysis of fit results without making any race/ethnic comparison were considered. Fit results of the model for the Hispanic group was provided earlier (Table 33) and fit results of the model for Blacks are provided below (Table 35).

Table 35: Fit results for JHo for the Black group

\begin{tabular}{|c|c|c|c|c|c|}
\hline IV & Data & \multicolumn{4}{|c|}{$\begin{array}{l}\text { Model } \\
\text { calc. } \mathrm{q}(\mathrm{DV} \mid \mathrm{IV})\end{array}$} \\
\hline $\mathrm{J}$ & freq & $\mathrm{Ho}=0$ & $\mathrm{Ho}=1$ & $\mathrm{p}$ (margin) & Ratio, $\mathrm{Ho}=1$ \\
\hline${ }^{\alpha} 1$ & 97 & 92.8 & 7.2 & 0.001 & 0.35 \\
\hline 2 & 241 & 78.0 & 22.0 & 0.54 & 1.08 \\
\hline${ }^{\beta} 3$ & 108 & 71.3 & 28.7 & 0.032 & 1.41 \\
\hline & & 79.6 & 20.4 & & \\
\hline
\end{tabular}

Note: ${ }^{\alpha}=$ protective $\&^{\beta}=$ risky states

In Blacks (Table 35), being employed $(\mathrm{J}=1)$ was related to lower rates of hospitalization while overnight hospitalization risk to those retired $(\mathrm{J}=2)$ was not significantly different from the marginal risk to the entire group. Those falling in job category 'other' $(\mathrm{J}=3)$ were at significantly higher risk of hospitalization compared to the marginal risk to the group. 


\section{Summary}

Comparing Whites with Hispanics, and Whites with Blacks, the suggested model for Whites was the independence model indicating that hospitalization was not related to any predictors in the White group. In Hispanics, gender and negative affect were independently related to overnight hospitalization. Being male and presence of negative affect increased risk of overnight hospitalization relative to the marginal risk, whereas, being female and presence of negative affect posed similar risk as the marginal risk to the group. For male Hispanics without negative affect, the risk was similar to the marginal group but for female Hispanics without negative affect, the risk was significantly lower than the marginal risk to the group. These suggest that presence of negative affect posed greater risk to males than females in the Hispanic group. In Blacks, job status was related to overnight hospitalization. Employed Blacks had lower risk of overnight hospitalization, and Blacks falling in job category 'other' had higher risk for overnight hospitalization, whereas, retired Blacks had similar risk to the marginal risk to the group. These results indicate that hospitalization varied by gender in Hispanics. In the Black group, hospitalization varied by employment status where being employed reduced risk and falling in job category 'other' $(\mathrm{J}=3)$ increased risk but being retired $(\mathrm{J}=2)$ posed similar risk as the marginal risk to the entire group.

\subsection{Cross-Sectional Path Analysis of Overnight Hospitalization}

Strengths of relationships between SES/demographic variables with the intermediate variables, and between the intermediate variables and overnight 
hospitalization were explored using cross-sectional data. This section provides results of path analysis for the overnight hospitalization outcome variable.

The research questions in this section were:

RQ6a: Do relationships between SES/demographic variables and intermediate variables differ by race/ethnicity?

RQ6b: Do relationships between intermediate variables and overnight hospitalization differ by race/ethnicity?

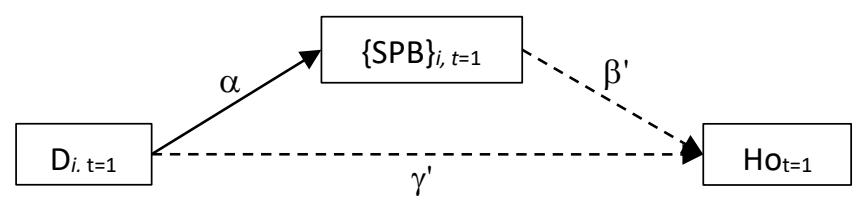

Figure 25: Cross-sectional path modeling of overnight hospitalization Note: $i=$ selected variable(s) \& $\mathrm{t}=$ time

Intermediate and SES/demographic variables associated with each other in this section were similar to the variables in the cross-sectional and longitudinal path models provided earlier (Section 6.2 and 6.4). SES/demographic to intermediate variable relationships were also strong in several cases (Table 36, $\alpha$ column). However, in all the path models, the effects of the selected intermediate variable on overnight hospitalization, controlling for the direct effect of the selected SES/demographic variable, were weak across groups (Table 36, $\beta^{\prime}$ column). These weak relationships also mirror the results in Section 6.5 where few variables were weakly related to overnight hospitalization. Given the weak indirect relationships, specifically, the $\beta^{\prime}$ path values, further elaboration of individual relationships by race/ethnicity is not provided in this study, but all variables and their path values are provided in Table 36 . 
Table 36: Uncertainty reductions for cross-sectional path models of overnight hospitalization

\begin{tabular}{|c|c|c|c|c|}
\hline Race/ethnicity & $\{\mathbf{S P B}\}$ factors & $\boldsymbol{\alpha}=\mathrm{T}(\mathrm{D}:\{\mathrm{SPB}\})$ & $\boldsymbol{\beta}^{\prime}=\mathrm{T}_{\mathrm{D}}(\{\mathrm{SPB}\}: \mathrm{Ho})$ & $\gamma^{\prime}=\mathrm{T}_{\{\mathrm{SPB}\}}(\mathrm{D}: \mathrm{Ho})$ \\
\hline \multirow{12}{*}{$\begin{array}{l}\text { White } \\
\text { vs } \\
\text { Hispanic }\end{array}$} & \multirow{3}{*}{ Social } & $\mathrm{I} \rightarrow \mathrm{Mar}$ & Mar $\rightarrow$ Ho & $\mathrm{I} \rightarrow \mathrm{Ho}$ \\
\hline & & 8.1 & 2.5 & 2.9 \\
\hline & & 8.6 & 1.5 & 1.8 \\
\hline & \multirow{4}{*}{ Psychological } & $\mathrm{J} \rightarrow$ Som & Som $\rightarrow$ Ho & $\mathrm{J} \rightarrow \mathrm{Ho}$ \\
\hline & & 5.6 & 0.3 & 0.2 \\
\hline & & $\mathrm{W} \rightarrow \mathrm{Neg}$ & $\mathrm{Neg} \rightarrow \mathrm{Ho}$ & $\mathrm{W} \rightarrow \mathrm{Ho}$ \\
\hline & & 4.2 & 2.6 & 2.6 \\
\hline & \multirow{5}{*}{ Behavioral } & $\overline{\mathrm{G} \rightarrow \mathrm{Alc}}$ & $\mathrm{Alc} \rightarrow \mathrm{Ho}$ & $\mathrm{G} \rightarrow \mathrm{Ho}$ \\
\hline & & 4.3 & 0.8 & 0.1 \\
\hline & & 6.7 & 1.2 & 2.2 \\
\hline & & $\mathrm{G} \rightarrow \mathrm{Smk}$ & $\mathrm{Smk} \rightarrow \mathrm{Ho}$ & $\mathrm{G} \rightarrow \mathrm{Ho}$ \\
\hline & & 5.9 & 0.4 & 1.5 \\
\hline \multirow{13}{*}{$\begin{array}{l}\text { White } \\
\text { vs } \\
\text { Black }\end{array}$} & \multirow{3}{*}{ Social } & $\mathrm{GI} \rightarrow \mathrm{Mar}$ & $\mathrm{Mar} \rightarrow \mathrm{Ho}$ & $\mathrm{GI} \rightarrow \mathrm{Ho}$ \\
\hline & & 13.2 & 2.1 & 2.0 \\
\hline & & 12.2 & 1.9 & 2.1 \\
\hline & \multirow{4}{*}{ Psychological } & $\mathrm{I} \rightarrow$ Som & $\mathrm{Neg} \rightarrow \mathrm{Ho}$ & $\mathrm{I} \rightarrow \mathrm{Ho}$ \\
\hline & & 4.1 & 0.4 & 0.2 \\
\hline & & EdJ $\rightarrow$ Som & Som $\rightarrow$ Ho & $\mathrm{EdJ} \rightarrow \mathrm{Ho}$ \\
\hline & & 8.1 & 1.8 & 2.3 \\
\hline & \multirow{6}{*}{ Behavioral } & $\overline{\mathrm{W} \rightarrow \mathrm{Ex}}$ & $\mathrm{Ex} \rightarrow \mathrm{Ho}$ & $\mathrm{W} \rightarrow \mathrm{Ho}$ \\
\hline & & 2.3 & 1.8 & 0.3 \\
\hline & & $\mathrm{G} \rightarrow \mathrm{Alc}$ & Alc $\rightarrow \mathrm{Ho}$ & $\mathrm{G} \rightarrow \mathrm{Ho}$ \\
\hline & & 6.1 & 0.3 & 0.2 \\
\hline & & $\mathrm{G} \rightarrow \mathrm{Bmi}$ & $\mathrm{Bmi} \rightarrow \mathrm{Ho}$ & $\mathrm{G} \rightarrow \mathrm{Ho}$ \\
\hline & & 2.8 & 0.03 & 0.1 \\
\hline \multirow{11}{*}{$\begin{array}{c}\text { Black } \\
\text { vs } \\
\text { Hispanic }\end{array}$} & \multirow{4}{*}{ Social } & $\mathrm{G} \rightarrow \mathrm{Mar}$ & Mar $\rightarrow$ Ho & $\mathrm{G} \rightarrow \mathrm{Ho}$ \\
\hline & & 5.3 & 0.6 & 0.6 \\
\hline & & $\mathrm{I} \rightarrow \mathrm{Mar}$ & $\mathrm{Mar} \rightarrow \mathrm{Ho}$ & $\mathrm{I} \rightarrow \mathrm{Ho}$ \\
\hline & & 8.6 & 1.5 & 1.8 \\
\hline & \multirow{4}{*}{ Psychological } & Ed $\rightarrow$ Som & Som $\rightarrow$ Ho & Ed $\rightarrow$ Ho \\
\hline & & 5.1 & 1.5 & 1.1 \\
\hline & & $\mathrm{W} \rightarrow \mathrm{Neg}$ & $\mathrm{Neg} \rightarrow \mathrm{Ho}$ & $\mathrm{W} \rightarrow \mathrm{Ho}$ \\
\hline & & 4.2 & 2.7 & 2.7 \\
\hline & \multirow{3}{*}{ Behavioral } & $\overline{\mathrm{G} \rightarrow \mathrm{Alc}}$ & $\mathrm{Alc} \rightarrow \mathrm{Ho}$ & $\overline{\mathrm{G} \rightarrow \mathrm{Ho}}$ \\
\hline & & 7.3 & 0.6 & 0.6 \\
\hline & & 6.7 & 1.2 & 2.3 \\
\hline
\end{tabular}

Note: D variables, $\{$ SPB $\}$ variables, and Ho variables were from Time 1.

\subsection{Longitudinal Analysis of Overnight Hospitalization}

Socioeconomic/demographic variables at the first time point together with social, psychological and behavioral factors at the second time point were used to predict 
overnight hospitalization (Ho) at the third time point. Each of the following sections provides results comparing pairs of race/ethnic groups.

The research questions in this section were:

RQ7a: How do SES/demographic variables at the first time point and social integration, variables, psychological variables, and behavioral variables at the second time point interact in predicting overnight hospitalization at the third time point?

RQ7b: Do such predictions differ by race/ethnic groups?

Table 37 provides results of models in this section. In White vs Hispanic, and Black vs Hispanic, the best models suggested were the independence models (models not shown). Further, the models comparing Whites and Blacks were simple.

Table 37: Racial and ethnic comparison of longitudinal models

\begin{tabular}{|c|l|}
\hline Race/ethnicity & Model \\
\hline White & -- \\
vs & - \\
Hispanics & - \\
\hline $\begin{array}{c}\text { White } \\
\text { vs } \\
\text { Blacks }\end{array}$ & SomHo \\
\cline { 2 - 2 } Blacks \\
vs \\
Hispanics & ExHo \\
\hline
\end{tabular}

\section{(a) Whites versus Hispanics}

In both White and Hispanic groups, the SES/demographic, social, psychological, and behavioral factors were unrelated to overnight hospitalization at the third time point (IV:Ho). 


\section{(b) Whites versus Blacks}

Somatic symptoms at the second time point predicted overnight hospitalization at the third time point in the White group. However, percent uncertainty reduction in overnight hospitalization was small. In the Black group, physical activity at the second time point was associated with overnight hospitalization at the third time point but physical activity was not greatly informative of overnight hospitalization as indicated by the small percent uncertainty reduction $(\% \Delta \mathrm{U}(\mathrm{Ho})=2.3 \%)$ as shown in Table 38 .

Table 38: Models out loops for White and Black groups

\begin{tabular}{|l|l|r|r|}
\hline Race/ethnicity & \multicolumn{1}{|c|}{ Model } & $\Delta \mathrm{df}$ & $\% \Delta \mathrm{U}(\mathrm{Ho})$ \\
\hline White & SomHo & 1 & 0.99 \\
\hline Black & ExHo & 2 & 2.33 \\
\hline
\end{tabular}

Fit results for the White group indicated that presence of somatic symptoms appeared to pose significantly higher risk compared to the marginal risk to the entire group although the effect sizes were close. Absence of somatic symptoms was marginally protective from overnight hospitalization, however.

Table 39: Fit results for SomHo for the White group

\begin{tabular}{|c|c|c|c|c|c|}
\hline \multirow{2}{*}{ IV } & Data & \multicolumn{3}{|c|}{$\begin{array}{c}\text { Model } \\
\text { calc. } \mathrm{q}(\mathrm{DV} \mid \mathrm{IV})\end{array}$} \\
\hline Som & freq & Ho=0 & Ho=1 & $\mathrm{p}$ (margin) & Ratio, Ho=1 \\
\hline 0 & 349 & 77.7 & 22.4 & 0.064 & 0.84 \\
\hline$\beta 1$ & 298 & 68.1 & 31.9 & 0.045 & 1.19 \\
\hline & 647 & 73.3 & 26.7 & & \multicolumn{1}{c}{} \\
\cline { 2 - 6 } & &
\end{tabular}

Note: ${ }^{\beta}=$ risky state

Blacks who did not engage in physical activity $(\mathrm{Ex}=0)$ had significantly higher risk of overnight hospitalization at the 0.05 level compared to the marginal risk to the group but the effect sizes were close. However, the risk to Blacks who engaged in 
moderate physical activity was similar to the marginal risk to the group. Frequent physical activity was associated with significantly lower risk (0.51) of overnight hospitalization in Blacks (Table 40).

Table 40: Fit results for ExHo for the Black group

\begin{tabular}{|c|c|c|c|c|c|}
\hline \multirow{2}{*}{ IV } & Data & \multicolumn{2}{|c|}{$\begin{array}{c}\text { Model } \\
\text { calc. q(DV|IV) }\end{array}$} \\
\hline Ex & freq & Ho=0 & Ho=1 & $\mathrm{p}$ (margin) & Ratio, Ho=1 \\
\hline$\beta_{0}$ & 422 & 69.7 & 30.3 & 0.028 & 1.18 \\
\hline 1 & 109 & 79.8 & 20.2 & 0.192 & 0.79 \\
\hline$\alpha_{2}$ & 108 & 87.0 & 13.0 & 0.002 & 0.51 \\
\cline { 2 - 6 } & 639 & 74.3 & 25.7 & \multicolumn{2}{c}{} \\
\cline { 2 - 6 } & & &
\end{tabular}

Note: ${ }^{\alpha}=$ protective $\&^{\beta}=$ risky states

\section{(c) Blacks versus Hispanics}

Search for models with loops in Black versus Hispanic groups also suggested that no SES/demographic, social, psychological, and behavioral factors were related to overnight hospitalization at the third time point. As above, presentation of further results was omitted.

\section{Summary}

Overall, SES/demographic, psychological, social, and behavioral factors were not related to overnight hospitalization across groups. Where few variables appeared to predict, the association was pretty weak, indicating near absence of longitudinal relationships between the predictors and overnight hospitalization in this data across race/ethnic groups. 


\subsection{Longitudinal Path Analysis of Overnight Hospitalization}

This section followed the steps for section 6.6 but used data for social integration, psychological and behavioral variables from the second time point and for overnight hospitalization from the third time point.

The research questions in this section were:

RQ8a: Do relationships between SES/demographic variables at the first time point and intermediate variables at the second time point differ by race/ethnicity?

RQ8b: Do relationships between intermediate variables at the second time point and overnight hospitalization at the third time point differ by race/ethnicity?

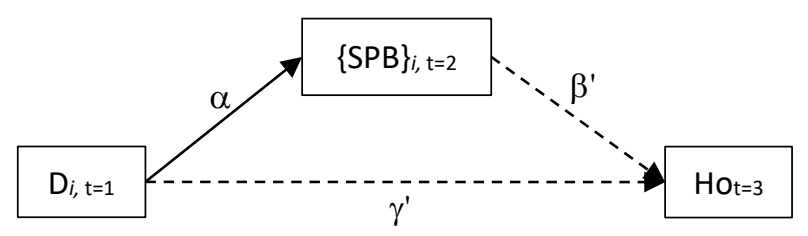

Figure 26: Longitudinal path modeling of overnight hospitalization Note: $i=$ selected variable(s) \& $\mathrm{t}=$ time

Similar to results in Section 6.6, several of the relationships between the selected SES/demographic variables at the first time point and the selected intermediate variables at the second time point were strong ( $\alpha$ column, Table 41). However, the intermediate variables to the overnight hospitalization variable ( $\beta^{\prime}$ column) path values were weak, indicating lack of evidence for hypothesized indirect association between SES/demographic and overnight hospitalization via the $\mathrm{S}, \mathrm{P}$, and B intermediate variables. Results are provided in Table 41 but further elaboration of these path values is 
not provided because of the weak indirect associations across groups, particularly weak \{SPB $\}$ to overnight hospitalization (Ho) (see $\beta^{\prime}$ column, Table 41).

Longitudinal relationships between SES/demographic and the intermediate variables were similar to the relationships described with physical impairment status as outcome variable. However, adjusted paths were weaker indicating that SES/demographic and intermediate variables were not related to overnight hospitalization. This was also consistent with the weak results found in the previous section (Section 6.6).

In summary, as in the path analysis values in previous sections, overall relationships between SES and intermediate variables were often at least moderately strong. However, adjusted path values were weak in most cases indicating lack of direct or indirect association between SES/demographic variables and overnight hospitalization. It, therefore, appears that associations with potential to result in race/ethnic based differences were not prominent. The only source of race/ethnic differences in overnight hospitalization may be due to the different variables selected and the means by which these variables have their impact within various race/ethnic groups. Where variables were identical, sizes of percent uncertainty reductions for race/ethnic groups compared were not substantially different. 
Table 41: Uncertainty reductions for longitudinal path models of overnight hospitalization

\begin{tabular}{|c|c|c|c|c|}
\hline Race/ethnicity & $\{$ SPB $\}$ factors & $\boldsymbol{\alpha}=\mathrm{T}(\mathrm{D}:\{\mathrm{SPB}\})$ & $\boldsymbol{\beta}^{\prime}=\mathrm{T}_{\mathrm{D}}(\{\mathrm{SPB}\}: \mathrm{Ho})$ & $\left.\gamma^{\prime}=\mathrm{T}_{\{\mathrm{SPB}\}}\right\}(\mathrm{D}: \mathrm{Ho})$ \\
\hline \multirow{13}{*}{$\begin{array}{l}\text { White } \\
\text { vs } \\
\text { Hispanic }\end{array}$} & \multirow{3}{*}{ Social } & $\mathrm{I} \rightarrow \mathrm{Mar}$ & Mar $\rightarrow$ Ho & $\mathrm{I} \rightarrow \mathrm{Ho}$ \\
\hline & & 7.4 & 1.9 & 2.8 \\
\hline & & 8.5 & 2.0 & 2.6 \\
\hline & \multirow{4}{*}{ Psychological } & $\mathrm{J} \rightarrow$ Som & Som $\rightarrow$ Ho & $\mathrm{J} \rightarrow \mathrm{Ho}$ \\
\hline & & 5.6 & 0.6 & 0.6 \\
\hline & & $\mathrm{J} \rightarrow \mathrm{Pos}$ & Pos $\rightarrow \mathrm{Ho}$ & $\mathrm{Pos} \rightarrow \mathrm{Ho}$ \\
\hline & & 3.6 & 0.2 & 0.9 \\
\hline & \multirow{6}{*}{ Behavioral } & $\mathrm{G} \rightarrow \mathrm{Ex}$ & $\mathrm{Ex} \rightarrow \mathrm{Ho}$ & $\mathrm{G} \rightarrow \mathrm{Ho}$ \\
\hline & & 3.7 & 1.5 & 0.2 \\
\hline & & $\mathrm{G} \rightarrow \mathrm{Alc}$ & Alc $\rightarrow$ Ho & $\mathrm{G} \rightarrow \mathrm{Ho}$ \\
\hline & & 8.4 & 2.7 & 2.8 \\
\hline & & $\mathrm{G} \rightarrow \mathrm{Smk}$ & $\mathrm{Smk} \rightarrow \mathrm{Ho}$ & $\mathrm{G} \rightarrow \mathrm{Ho}$ \\
\hline & & 5.6 & 0.7 & 0.9 \\
\hline \multirow{11}{*}{$\begin{array}{l}\text { White } \\
\text { vs } \\
\text { Black }\end{array}$} & \multirow{3}{*}{ Social } & $\mathrm{GI} \rightarrow \mathrm{Mar}$ & Mar $\rightarrow$ Ho & $\mathrm{GI} \rightarrow \mathrm{Ho}$ \\
\hline & & 13.1 & 1.5 & 1.5 \\
\hline & & 12.4 & 2.2 & 2.2 \\
\hline & \multirow{4}{*}{ Psychological } & $\mathrm{J} \rightarrow$ Som & $\mathrm{Neg} \rightarrow \mathrm{Ho}$ & $\mathrm{I} \rightarrow \mathrm{Ho}$ \\
\hline & & 3.0 & 1.5 & 0.6 \\
\hline & & EdJ $\rightarrow$ Som & Som $\rightarrow$ Ho & EdJ $\rightarrow$ Ho \\
\hline & & 6.1 & 0.1 & 0.2 \\
\hline & \multirow{4}{*}{ Behavioral } & $\mathrm{G} \rightarrow \mathrm{Smk}$ & Smk $\rightarrow$ Ho & $\mathrm{G} \rightarrow \mathrm{Ho}$ \\
\hline & & 1.8 & 1.0 & 0.5 \\
\hline & & $\mathrm{G} \rightarrow \mathrm{Alc}$ & $\mathrm{Alc} \rightarrow \mathrm{Ho}$ & $\mathrm{G} \rightarrow \mathrm{Ho}$ \\
\hline & & 4.7 & 0.2 & 0.2 \\
\hline \multirow{13}{*}{$\begin{array}{c}\text { Black } \\
\text { vs } \\
\text { Hispanic }\end{array}$} & \multirow{4}{*}{ Social } & $\mathrm{G} \rightarrow \mathrm{Mar}$ & Mar $\rightarrow$ Ho & $\mathrm{G} \rightarrow \mathrm{Ho}$ \\
\hline & & 6.2 & 1.6 & 0.8 \\
\hline & & $\mathrm{I} \rightarrow \mathrm{Mar}$ & Mar $\rightarrow$ Ho & $\mathrm{I} \rightarrow \mathrm{Ho}$ \\
\hline & & 8.5 & 2.0 & 2.6 \\
\hline & \multirow{4}{*}{ Psychological } & Ed $\rightarrow$ Som & Som $\rightarrow$ Ho & $\mathrm{Ed} \rightarrow \mathrm{Ho}$ \\
\hline & & 5.8 & 0.8 & 0.1 \\
\hline & & $\mathrm{J} \rightarrow \mathrm{Pos}$ & $\mathrm{Pos} \rightarrow \mathrm{Ho}$ & $\mathrm{J} \rightarrow \mathrm{Ho}$ \\
\hline & & 3.6 & 0.2 & 0.9 \\
\hline & \multirow{5}{*}{ Behavioral } & $\mathrm{G} \rightarrow$ Alc & $\mathrm{Alc} \rightarrow \mathrm{Ho}$ & $\overline{\mathrm{G} \rightarrow \mathrm{Ho}}$ \\
\hline & & 7.1 & 0.5 & 0.3 \\
\hline & & 8.4 & 2.7 & 2.8 \\
\hline & & $\mathrm{G} \rightarrow \mathrm{Smk}$ & $\mathrm{Smk} \rightarrow \mathrm{Ho}$ & $\mathrm{G} \rightarrow \mathrm{Ho}$ \\
\hline & & 5.6 & 0.9 & 0.7 \\
\hline
\end{tabular}

Note: D variables were from Time 1, $\{\mathrm{SPB}\}$ variables from Time 2, and Ho variable from Time 3.

\section{Missing Data in RA}

Missing data were excluded in the RA analysis. However, missing data can also be analyzed in the OCCAM implementation of RA by assigning missing as another state 
(value) of a variable. Thus, the relationship of missing data with other IV states and effects on the outcome variables can be analyzed when necessary. This is a potential future avenue for further analysis of data with missing values using RA. 


\section{CHAPTER 7.0: STRUCTURAL EQUATION MODELING: DISCUSSION AND CONCLUSION}

This section provides discussions and conclusions for the SEM results. Indirect relationships between four SES variables (income, wealth, employment status, and education level) and two outcome variables (physical impairment and overnight hospitalization) via social, psychological and behavioral factors were compared and differences highlighted among Whites, Blacks and Hispanics.

\section{Physical Impairment Status}

\subsection{Means and Correlations}

SES: Social organizational systems in the United States were created and are maintained by the majority group (non-Hispanic Whites) primarily for its own benefit. Competing for scarce resources in such an environment for minorities was and continues to be challenging. Given the historical facts, non-Hispanic Whites in the United States were, on average, expected to fall in higher SES position. Consistent with previous studies (Krieger et al., 1999; Williams et al., 2012), mean income, mean wealth, education levels and proportion of employed respondents in non-Hispanic Whites were higher than in Blacks and Hispanics in the current study. Inter-correlations among SES variables were stronger for the White group indicating, for instance, that the return of investment in education for Whites may be higher income and greater wealth accumulation compared to similar levels of education in Blacks and Hispanics. Age was positively correlated with both total household income and total wealth in the White group. This may suggest that Whites earn higher income and accumulate greater wealth 
levels than their Black and Hispanic counterparts. Income, in the present study, was computed from several sources including income from regular employment, annuities, Social Security benefits, and income from unemployment status or workers compensation. Income from regular employment and income from annuities may be higher for Whites indicating positive correlation between total household income and age. In Blacks and Hispanics, total household income and total wealth were not significantly correlated with age, however. Lack of significant positive correlation between age and income, and between age and wealth, in the Black and Hispanic groups may indicate that income and wealth disparities between Whites and minorities are lasting. One similar trend across groups was that education and employment were negatively correlated with age. Females had lower SES across groups except in Blacks where females had higher mean education level than their male counterparts.

Social integration: Mean differences in social integration scores among the three race/ethnic groups showed that Whites had higher scores than Blacks and Hispanics which was also consistent with the hypothesis in this study. Higher SES individuals are more likely married or cohabiting, more likely to have friends in the neighborhood or visit friends (Marmot et al., 1991) and more likely to engage in wider non-stressful social activities that form a higher social integration score when combined. Income and wealth were significantly correlated with social integration at the first time point and at the second time point across all groups, indicating these possibly contribute to levels of social stability over time. 
Social integration was negatively correlated with physical impairment across groups as hypothesized. It is likely that as social integration affects physical impairment, in turn, physical impairment may limit social integration, creating a complex web of reinforcing feedback mechanisms that were not part of this study. The benefits of social interactions or lack of interactions, thereof, operate at biological levels to impact physical well-being. In a review of dozens of studies, Uchino, Cacioppo, \& Kiecolt-Glaser, (1996) found that social support was related to physiological processes beneficial to health.

Psychological factors: Baseline mean depressive symptoms scores varied by race/ethnicity with Hispanics having higher depressive symptoms scores followed by Blacks and Whites. Whites' depressive symptoms scores were sizably lower than the scores for Hispanics and Blacks which may be a reflection of higher SES or the fact that this group forms the majority and does not experience stressful environments due to implicit or explicit discrimination based on race/ethnicity. Mean depressive symptoms in each group remained relatively steady over time, however. Minorities likely face widespread challenges that increase heightened vigilance which is known to be detrimental to health through physiological processes (McEwen \& Stellar, 1993; Sapolsky, 1998, 2004; Peter Sterling \& Eyer, 1988). The result that depressive symptoms were lower in respondents with higher SES supported the hypothesis in concordance with findings from previous studies (Pearlin et al., 1981; Pearlin \& Schooler, 1978) demonstrating that higher SES provides the necessary resources to cope with stressors or simply acts as a protective factor from psychological stressors. 
Behavioral factors - body mass index (BMI): Although some studies (Laaksonen et al., 2003; Wray et al., 2006) categorize BMI within behavioral factors, there is evidence that under sustained stress, metabolic mechanisms function differently, potentially forcing the body to store excess fat that adversely impacts trajectory of physical impairment status via chronic diseases. In the present study, the trends in mean differences in BMI were also consistent with previous findings (Flegal, Carroll, Kit, \& Ogden, 2012) where Blacks had higher BMI scores followed by Hispanics and Whites. In this study, BMI remained steady from the first time point to the second time point within each race/ethnic group. Respondents in this study were older, so, variability in BMI from the first time point to the second time point may be small while larger intergroup differences persist because considerable shifts in BMI may not occur in older age.

Behavioral factors - alcoholic beverage consumption (drinking): Mean alcohol consumption among Whites was nearly two times more than that among Blacks and Hispanics contrary to the hypothesis in this study. The drinking variable in the HRS dataset may not reflect problem drinking (i.e., alcohol abuse), rather, it perhaps reveals respondents' higher SES position. Overall, contrary to the hypothesis in the study, wealth, income, education and employment levels were positively correlated with alcoholic beverage consumption. These results were consistent with findings from other studies that showed higher SES individuals, on average, consumed more alcoholic beverages (Laaksonen et al., 2003; Marmot et al., 1991). In the present study, the positive correlations between SES variables and average alcoholic beverage consumption levels, 
thus, may be viewed to constitute beneficial social (casual) drinking rather than alcohol abuse.

Physical impairment: Dependence on others to perform activities of daily living gradually increases with age. But, individuals in higher SES position tend to have slower decline in performing activities of daily living over time as shown in this study. Just as differences in SES occur along racial/ethnic lines, physical impairment levels vary across racial/ethnic groups where lower SES scores are correlated with higher physical impairment scores at successive time points. Thus, mean physical impairment scores for Blacks and Hispanics were similar but almost two times higher than the mean physical impairment scores for Whites consistent with the hypothesis. Physical impairment scores from the first time point to the second and the third time point, in the current study, increased for all race/ethnic groups, but differences persisted with Blacks and Hispanics experiencing higher decline in physical health status. These suggest that race/ethnic differences in physical impairment continue in old age as has been found in other studies of older Americans (August \& Sorkin, 2010).

Overnight hospitalization: Baseline proportion of hospitalizations in Whites, Blacks and Hispanics were essentially similar but increased slightly over time with greater increase in the Black group. It should be noted that a large portion of the sample in this study would be expected to have some form of insurance coverage through Medicare. But although inter-group variation in medical care use may shrink due to Medicare, differences between groups may persist because of life long practice of 
differential responses to medical care need by race/ethnicity. To elaborate, studies have shown that African Americans delay seeking medical care for several reasons including lack of access due to structural barriers or due to insurance and lack of trust in the healthcare system.

\section{Differences in Indirect Path Coefficients by Race/Ethnicity}

Involuntary unemployment for individuals below the retirement age poses social and psychological challenges such that social interactions may be unpleasant and uncertainty induced anxiety may be elevated. Unemployment among men has been shown to predict depressive symptoms (Linn, Sandifer, \& Stein, 1985) and poverty presses individuals to seek escape routes from stressors by engaging in activities counter to healthy behaviors such as smoking and sedentary lifestyles. Although there was strong inter-correlation among the SES variables, adjusting for the effects of other SES variables in the regression equations may attenuate individual path coefficients.

In the current study, proportion of respondents employed across groups was small which may be due to respondents being over the age of 65 as indicated by the strong negative correlation between age and employment status in the White and Black groups. Results of the lagged path models indicated that employment status at the first time point did not significantly predict social integration at the second time point. However, employment status was significantly inversely related to depressive symptoms at the second time point and physical impairment at the third time point. Retired Americans, however, earn income from various sources such as social security or dividends from 
various investments (Kaplan et al., 1987) that potentially contribute to differential levels of social integration by maintaining social connections created throughout life, buffering effects of psychological stressors, and engaging in health promoting behaviors. These differences in social integration, psychological status and behavioral factors are likely associated with physical health status such that wealthier individuals form supportive social structures and are protected from scarcity-induced stressors, and high SES individuals may be able to gain access to resources for physical activity and reduce impact of stressors via such outlets.

Non-Hispanic Whites earn higher income and accumulate greater wealth, a disparity that persists in old age as indicated by strong positive correlations of age with income and wealth in the White group but not in the Black group or Hispanic group. Relative poverty, that is, falling in lower social hierarchy has adverse health consequences for the lower SES group as shown in the Whitehall studies of British civil servants (Marmot et al., 1991) and animal studies (Sapolsky, 1998, 2004). The disadvantages due to relatively inferior levels of income and wealth among race/ethnic minorities that last over the life course manifest as disproportionately higher levels of adverse health statuses among these groups.

In concordance with previous studies, results in the current study showed that wealth was inversely related to physical impairment. Consistent with the hypothesis in this study, higher education was related to lower physical impairment in Whites but not in Hispanics or Blacks. Just as Blacks earn lower income for similar education level 
compared to Whites, Blacks at comparable levels of education also tend to have lower self-rated health (Farmer \& Ferraro, 2005), an indication of consistency in the differential relationship between SES and health status across groups controlling for education.

\subsection{SES-Social Factors-Physical Impairment Link}

Constituents of the social integration index such as being married which tend to be higher among men in higher job grades (Marmot et al., 1991), volunteering in organizations and having good friends in the neighborhood are higher among Whites (Kaplan, 1995) which was also supported by the present study. Being near family or friends and falling in lower SES position relative to other groups likely complicates determination of effects of the relationships because of possible entanglement of negative interactions and supportive interactions. Previous studies, using the HRS data and a similar measure of the social integration index variable, also showed that better social integration was related to proper metabolic function (Yang et al., 2013), which may slow down physical impairment. In a related study, using similar social integration index from the HRS data, others (Ertel, Glymour, \& Berkman, 2009) also showed rapid memory decline among the least integrated respondents further providing evidence of the effects of social integration on physical as well as cognitive decline in a national scale study.

In the present study, higher income and wealth predicted higher social integration scores across groups but social integration was significantly negatively related to physical impairment only in the White group supporting the hypothesized relationships. These positive relationships between the two SES variables and social integration, and the 
negative relationship between social integration and physical impairment, appear to conform to the cumulative advantage/disadvantage theory over the life course. The direct effect of education on social integration was not significant, however, possibly attenuated by the presence of the other SES variables in the models.

Analysis of the SES-physical impairment link in the present study indicated that only in the White group did income and wealth indirectly predict physical impairment but the correlational relationships in the other groups were as hypothesized. These results support the notion that higher income and wealth afford better social support systems which are inversely related to physical impairment, thus potentially generating race/ethnic disparities in physical impairment status over the life course. Underlying these high level relationships are complex physiological mechanisms resulting from chronic stress due to unpredictability of events and lack of control over events among lower SES groups, that is, minorities in the United States (Lynch et al., 1997; Williams, 1999) with the exception of Asian Americans. None of the SES variables were indirectly related to physical impairment in the Black or Hispanic groups. The notion was that higher income and wealth enabled individuals to create and stabilize qualitatively better and quantitatively more social connections creating better living conditions consistent with results from previous studies (Berkman \& Syme, 1979; House et al., 1988; McLeod \& Kessler, 1990; Taylor \& Seeman, 1999; Whelan, 1993). Results in this study point to the protective effects of income and wealth against physical impairment via social integration. 
Follow up multiple group path equality tests using nested models indicated that fit results in the constrained and freely estimated models were not significantly different, suggesting that paths were not different across groups.

Growth curve model results—social integration: Path models with observed variables and latent growth curve models vary in important ways. Lagged path models provide information about the stability of rank order of the variables in the sample while latent growth curve models provide trajectory of inter-individual and intra-individual variation. Additionally, latent growth curve modeling approach corrects for measurement error in estimating parameters. The goal of using latent growth curve models for the social integration variable was to examine how SES variables were related to the trajectory of social integration scores, and in turn, how the trajectory of social integration scores were related to the trajectory of physical impairment scores over time. Importantly, the model was used to examine whether SES was indirectly related to physical impairment growth factor and whether these relationships differed by race/ethnicity.

Decrease in social integration scores with age is inevitable because participation in activities that increase social integration scores as conceptualized in this study decline due to loss of friends, death of a spouse, inability to frequently hold conversations and engage in volunteering because of declining physical ability. In this study, mean growth factor for the social integration variable in the White group was negative and significant, but only marginally significant in Blacks, suggesting that, social integration scores 
declined significantly every two years for the White group and the Black group. In the Hispanic group, the trajectory of social integration was negative, also indicating a declining trajectory of social integration in this group although this decline did not reach statistical significance. Variations in living arrangements and frequency of social interactions in old age may differ by race/ethnicity which may explain differences in the trajectories across groups. That is, cultural tendencies in the Hispanic community may tend to be collectivist rather than individualist, a cultural feature that may benefit individuals in older age.

As in the path models above, SES was positively related to the social integration slope factor and negatively related to the physical impairment slope factor across groups. The trajectory of social integration predicted the trajectory of physical impairment, where, increase in the social integration slope factor was related to decrease in the physical impairment slope factor as hypothesized in this study. Indirect associations between SES and physical impairment slope factor showed that only in the White group did education and employment status indirectly predict the trajectory of physical impairment. Higher education and being employed significantly indirectly predicted physical limitations in Whites, indicating slower decline for this group. Conceptually, education can be seen as a useful tool in forming stable relationships that increase the social integration scores because higher education is likely related to communication skills that reduce conflict. It is important to note here that in the path model, income and wealth indirectly predicted physical impairment while in the latent growth curve model education and job status indirectly predicted physical impairment in the White group. 
None of the indirect effects of SES on the trajectory of physical impairment via the trajectory of social integration in the Black and Hispanic groups were significant. Thus, in both the lagged path model and latent growth curve model, results supported the notion that SES may operate to slow down trajectory of physical decline in the White group more than it does in the Black and Hispanic groups directly and indirectly through social integration. However, follow up analysis using nested models indicated paths were not different across groups. Thus, the differences in the relationships described above may be due to sample size or variability in SES scores in the White group.

\subsection{SES-Psychological Factors-Physical Impairment}

Consistent with other national scale studies, income, wealth, education and employment were inversely related to depressive symptoms in Whites, and depressive symptoms, in turn, were positively related to physical impairment status, where, increases in depressive symptoms were related to increases in physical impairment. Whites who rank higher in SES were better protected from experiencing psychological difficulties. In Blacks, only higher education and being employed significantly negatively predicted depressive symptoms but depressive symptoms scores did not significantly predict physical impairment status, controlling for other SES and demographic variables. In Hispanics, only increase in wealth and education significantly predicted decrease in depressive symptoms, and depressive symptoms scores were also positively related to physical impairment levels. It has been shown in this and previous studies (LaVeist, 2005; Williams, 1999) that education, income and wealth are strongly correlated and that for similar education level and job type, minorities and women earn less. Thus, the 
unfavorable social structures for minorities and women are multidimensional but the final outcome appears to be diminished health status relative to Whites.

Consistent with the hypothesis in the current study, analysis of indirect paths showed that three SES variables (wealth, employment and education) indirectly predicted physical impairment in Whites via depressive symptoms where increase in each of these SES variables was indirectly related to decrease in physical impairment. Overall, in Blacks and Hispanics, the indirect effect of SES on physical impairment status was not significantly different from zero except for the indirect effect of wealth on physical impairment in the Hispanic group. It is not clear if raising SES scores alone would completely change these results in the Black and Hispanic groups, however, because it has been found that neither race/ethnicity nor SES alone or together accounted for observed race/ethnic differences in health status (Williams, 1999). Real and perceived discrimination, because of one's race/ethnic background and gender may account for persistent stressors in the environment that may account for the remaining differences in health status among the various groups.

Growth curve models-psychological factors: The slope factor for depressive symptoms in each group appeared to decline over time although decline in the Hispanic group was not significant. Further, decline in depressive symptoms within the White group varied suggesting that decline in depressive symptoms among White respondents was not uniform. 
Slope growth factor for physical impairment in each group was positive and significant, indicating that in all race/ethnic groups, physical impairment significantly increased every two years. Trajectory of physical impairment within the White group and within the Black group varied, indicating decline in physical impairment within each group was not uniform. Decline in physical impairment over time within the Hispanic group did not vary, however. In the second order growth curve models, SES variables did not appear to be indirectly related to the slope factor for physical impairment through the slope factor for depressive symptoms in the White and Hispanic groups. However, in the Black group, income was positively related to depressive symptoms contrary to the hypothesis. The indirect effect of income on physical impairment slope factor was significant but in the opposite direction than hypothesized. This may likely be due to sampling error given the weak effect size.

\subsection{SES-Behavioral Factors-Physical Impairment}

Body Mass Index: Protective health behaviors are learned and this learning may come from one's home, formal education, neighborhood or information that one chooses to consume. To some extent, this learning is a privilege that is relatively easily accessible to individuals and communities in higher SES position. Higher SES affords the means of obtaining information on better dietary practices, on the benefits of physical activity, the knowledge to recognize the detrimental effects of excessive alcoholic beverage consumption and smoking. The relationships of these and other health promoting or health diminishing factors are complex, however. Although controversial in the epidemiological and public health disciplines, one of the indicators of health behaviors in 
populations is body mass index (BMI), and following previous studies BMI was used as a proxy for dietary behavior in this study.

Consistent with the hypothesis in this study, SES was negatively related to BMI as in previous studies (Laaksonen et al., 2003) but only wealth significantly predicted BMI in Whites. Further, higher BMI was significantly positively related to physical impairment in the White and Hispanic groups. Wealth was the only SES variable indirectly related to physical impairment via BMI in the White group but the effect size was small. None of the other SES variables were indirectly related to physical impairment in Whites. In Blacks, BMI was not related to physical impairment. It is possible that the composition of mass in the BMI differs by race/ethnicity reducing health risks associated with BMI (Flegal et al., 2012) for Blacks. Overall, these findings failed to support the hypothesized indirect association of SES with physical impairment through BMI. This may be due to lack of significant differences in BMI over time among older population or an indication of the ineffectiveness of BMI as an ideal measure.

Alcoholic beverage consumption (drinking): The assumption was that SES would be inversely related to drinking, and drinking would be positively related to physical impairment. However, path models in this study indicated that SES was positively related to drinking. This was consistent with previous findings, where, average alcoholic beverage consumption was related to being employed, particularly falling in higher grade of job category (Marmot et al., 1991), and income was positively related to alcohol consumption (Laaksonen et al., 2003), particularly among women. The positive 
relationship between SES and alcoholic beverage consumption indicates that respondents in higher SES position across groups, on average, consumed more alcoholic beverages but most of these effect sizes did not reach statistical significance except in the Hispanic group where income was significantly positively related to drinking. All other path coefficients from SES to drinking were not significant in each of the race/ethnic groups. In this study, the data failed to provide evidence in favor of differences in health disparities emanating from drinking levels but showed that in a national scale study SES was positively related to alcoholic beverage consumption.

\section{Overnight Hospitalization}

Attempting to explain hospitalization using SES or social, psychological or general behavioral variables is challenging. Overnight hospitalization may either be due to affordability because of one's higher SES position or it may indicate serious conditions requiring hospitalization. Because the sample was mostly over the age of 65 , respondents were likely covered by Medicare and Medicaid, leveling accessibility to medical treatment for covered services for racial/ethnic groups. Previous studies found that despite coverage after the age of 65 , medical care utilization by race/ethnicity revealed mixed results because minorities and non-Hispanic White women used fewer medical services and minority men had less outpatient surgery (Dunlop, Manheim, Song, \& Chang, 2002). Others (Eggers \& Greenberg, 2000) reported that Blacks, Hispanics and Native Americans had higher rates of hospitalization, but, Whites and Asian Americans had higher rates of revascularization perhaps an indication of differential treatment approaches. In the present study, baseline proportion of overnight hospitalizations by 
race/ethnicity hardly differed. There was slight increase, over time, in the proportion of overnight hospitalizations in each group with increase slightly higher for the Black group. Overall, SES variables were not strongly directly related to overnight hospitalization. In the Hispanic group, only income was significantly related to overnight hospitalization while in Whites, employment status predicted overnight hospitalization. The adjusted effects of other SES variables on overnight hospitalization across groups were weak.

\subsection{SES-Social Factors-Overnight Hospitalization}

In the present study, results showed a weak indirect relationship of SES with overnight hospitalization. Although the adjusted effects of SES on overnight hospitalization were negative, few direct paths were significant and the effect sizes were small as indicated above. Using the social integration index as a mediator, this study found no indirect relationship between each SES variable and overnight hospitalization via social integration. Social integration, therefore, may not have strong association with overnight hospitalization but may impact illnesses that do not require hospitalizations but accelerate physical impairment.

\subsection{SES-Psychological Factors-Overnight Hospitalization}

Hospitalizations due to psychiatric conditions were dropped in this study. Socioeconomic status variables at the first time point were negatively related to depressive symptoms at the second time point as indicated earlier. However, similar to the above results, the indirect path coefficients between SES and overnight hospitalization were weak across race/ethnic groups in this study. 


\subsection{SES-Behavioral Factors-Overnight Hospitalization}

Body mass index increases as SES decreases according to several national scale studies, and obesity is related to various chronic diseases. In this study, higher BMI was related to higher rates of overnight hospitalization in Whites and Blacks but not in Hispanics. Only income and wealth were indirectly related to overnight hospitalization in the White group, whereas, none of the SES variables were significantly indirectly related to overnight hospitalization through BMI in Blacks and Hispanics. The effect sizes for the significant indirect path coefficients in the White group were weak, suggesting, once again, that the driving factors of overnight hospitalizations may lie elsewhere.

As described earlier, the relationship of SES to alcohol consumption was positive contrary to the hypothesis in this study. But alcoholic beverage use was positively related to overnight hospitalization in the White group. The findings in this study failed to support the hypothesized indirect relationships between SES and overnight hospitalization through alcoholic beverage consumption in the White group because the sign of the product of the indirect path coefficients (positive) was opposite of the sign of the direct path coefficient (negative) of SES on overnight hospitalization. In Blacks, although BMI was positively related to overnight hospitalization, the indirect path coefficient was not significant. It is essential to note that all indirect effect sizes were weak indicating lack of strong relationships between SES and overnight hospitalization in this racially and ethnically diverse population of older Americans. Indeed, multiple group analysis of nested models indicated that paths were not different across groups. 


\section{Summary}

The present study contributed to the ongoing research attempting to identify the possible pathways by which SES is related to physical health status and medical care use in the United States. Several hypotheses in this study were supported in that SES was indirectly related to physical health status and overnight hospitalization rates via social, psychological and behavioral factors. Higher income, wealth, education and being employed positively impact levels of social integration, reduce psychological problems and promote better health behaviors. These social, psychological and behavioral factors, in turn, reduce growth rates of physical impairment due to chronic diseases or other health factors that generate race/ethnic disparities in physical health status and overnight hospitalizations.

Direction of effects, that is, signs between the predictor and the intermediate outcome, the intermediate outcome and the final outcome, as well as the predictor and the final outcome, were consistent with the hypotheses in this study with few exceptions. The exceptions include the positive relationships of SES with alcoholic beverage consumption, indicating that in a national scale study, alcoholic beverage consumption signaled higher SES status rather than alcohol abuse. Socioeconomic status was not significantly indirectly related to physical impairment through BMI except for the indirect path from wealth to physical impairment status in the White group.

Growth curve models provided trajectories of physical impairment status, social integration, depressive symptoms and the indirect effects of SES variables on physical 
impairment by race/ethnic group via social integration and depressive symptoms (psychological variables). Differences in the trajectory of physical impairment and social integration were evident, where, both social integration and physical impairment declined over time except for non-significant decline of social integration scores in the Hispanic group that may reflect cultural practices and living arrangements that slow down decline of social integration in this ethnic group. Among higher SES Whites, decline in social integration and increase in physical impairment varied significantly, indicating wealthier Whites had slower decline in social integration and slower increase in physical impairment than non-wealthier Whites. Similarly, increase in physical impairment among wealthier Blacks and Hispanics was slower than increase in physical impairment among less wealthier Blacks and Hispanics.

Given the nature of the sample and the similar sizes of indirect effects in other studies (Molina et al., 2013), these small variations potentially increase group differences in health status and healthcare utilization over time.

\section{Conclusions}

The discussions above showed that, overall, results in this study supported the hypothesized correlational relationships of the conceptual model that motivated this study. It is important to note that nearly all paths from SES to the proposed mediators (social, psychological, and behavioral factors), and from the mediators to physical impairment status were consistent with the hypothesized relationships. Further, in the White group, income, wealth, education level, and employment status appeared to have 
higher protective effects on physical impairment via social integration. In the path model with psychological factors as the mediator, in Whites, increase in each SES variable appeared to be related to decrease in physical impairment via decrease in depressive symptoms (psychological factors). Higher SES was positively related to alcoholic beverage consumption contrary to the hypothesized negative relationship. Thus, increase in SES was related to increase in physical impairment through increase in alcoholic beverage consumption, indicating a suppression effect (MacKinnon, Krull, \& Lockwood, 2000). SES was negatively related to BMI, and BMI was, in turn, positively related to physical impairment, consistent with the hypothesized relationships. Effect sizes for the indirect relationships were small, however. The indirect relationships between SES and overnight hospitalization were also consistent with the hypothesized relationship except for the relationship of income and wealth with overnight hospitalization via alcoholic beverage consumption, as described above. The relationships were weaker than when physical impairment was used as the health outcome variable, perhaps suggesting that other variables, such as chronic diseases, may be more pertinent predictors of overnight hospitalization.

Indirect effect sizes were small, however, and several reasons may have contributed to these small effect sizes across groups. First, path coefficients were all less than one, and because the size of the indirect effect is a product of individual path coefficients, sizes of indirect effects will necessarily be small (MacKinnon, 2008). Second, using several variables, this study examined and compared predictive paths by 
race/ethnicity, rather than comparing mean differences, so race/ethnic differences in predictive paths may be more subtle than differences in means.

\section{Missing Data}

Physical impairment status and overnight hospitalization were the main outcome variables. While physical impairment was treated as a continuous variable, overnight hospitalization was a binary variable. For non-normal or slightly non-normal data with missing values, using the MLR estimator in Mplus provides robust parameter estimates. With categorical outcomes the default estimator in Mplus is the weighted least squares mean-variance adjusted estimator (WLSMV). In the presence of missing data, WLSMV uses pairwise present data and is believed to provide more efficient estimates than using listwise deletion when dependent variables are specified as categorical in the Mplus program, but this estimator may not provide estimates that are as accurate as a full maximum likelihood approach if the data are not MAR (Asparouhov \& Muthén, 2010).

The patterns of missing data were similar across groups. Blacks and Hispanics had slightly more percent data missing relative to Whites. In each group, lower SES respondents appeared to be more likely to have missing data but the correlations between missing data indicator and each of these SES variables were not significant in almost all cases. Age, however, was positively related to data being missing which may not be anomalous. Results of the missing data analysis indicated that missing data appeared to be due to attrition mainly but also indicated cases with arbitrary missing patterns. Therefore, mainly, a combination of factors including age and low SES appeared to be 
related to missing data in this study, but other sources also appear to have minor impact on missing data.

The sample size for each group was large and using covariates including income, wealth, education, employment status, age and gender likely addressed some of the issues in missing data assumptions (Enders, 2010; Schafer \& Graham, 2002). Together with these strengths, the study also used full information maximum likelihood that provides robust standard errors for data with missing values. 


\section{CHAPTER 8.0: RECONSTRUCTABILITY ANALYSIS (RA): DISCUSSION AND CONCLUSION}

The RA method uses a search heuristic to extract informative relationships between variables. In this study, the RA method was used in hypotheses constrained exploratory and confirmatory analyses. The terms hypotheses constrained are used in the sense that in the neutral system, analyses were restricted to associations between SES/demographic variables and social variables (also between psychological and behavioral variables), rather than, for instance, among SES/demographic variables. It is distinct from traditional regression based methods such that in a directed system (where IV/DV distinctions are made) with one dependent variable and several predictors, the method examines and compares each variable's contribution to uncertainty reduction in the dependent variable, alone or via interaction with other independent variables. The most important variables are selected and a quantity indicating percent uncertainty reduction due to these important variables is provided. For models specifying independent and dependent variables, fit analyses (similar to log-linear analysis of contingency tables) of the selected models and relative risk between groups for combinations of composite IV states were examined. Another feature that distinguishes the RA method from confirmatory statistical methods is that in the OCCAM implementation of RA, the method conducts exploratory analysis suggesting variables associated with each other where there is no IV/DV distinction made, for instance, between SES/demographic variables and psychological variables. This type of 'neutral system analysis' was used to identify SES/demographic variables that were associated 
with intermediate variables (i.e., $\{$ SPB $\}$ variables) before carrying out directed system analysis investigating the effects of selected SES/demographic variables on associated intermediate variables. The next sections provide discussion of several RA results.

\section{Physical Impairment Status}

\subsection{Cross-Sectional and Longitudinal Analysis of Physical Impairment Status}

This section discusses selected cross-sectional and longitudinal results for direct prediction of physical impairment. In cross-sectional models (see Chapter 6, Table 14), analyses showed differences in selected predictor variables across races and ethnic groups. Cross-sectional models were more complex than longitudinal models (compare Table 14 and Table 22, Chapter 6). Interaction effects, in the conventional sense, did not feature in cross-sectional or longitudinal models except in one cross-sectional model for the Black group. Although models showed single predictors, uncertainty reduction in physical impairment due to the selected single predictive variables is not additive as displayed by fit tables. This indicates that the separate predictors do interact but not in the familiar and conventional way that is normally labeled an 'interaction effect'.

Cross-sectional models: Only one SES variable (employment status), one social variable (volunteering in organizations), two behavioral variables (BMI and exercise) appeared in the cross-sectional models. All three psychological factors appeared in the cross-sectional models (Table 42) but several other SES/demographic, social and behavioral factors did not appear in the cross-sectional models. 
Table 42: SES/demographic, social, psychological and behavioral variables in/not in cross-sectional models predicting physical impairment

\begin{tabular}{|c|c|c|c|c|}
\hline $\begin{array}{l}\text { Variable in } \\
\text { non-path } \\
\text { Model? }\end{array}$ & SES/Demo. & Social & Psychological & Behavioral \\
\hline Yes & - Job status & - Volunteering & $\begin{array}{l}\text { - Somatic symptoms } \\
\text { - Negative affect } \\
\text { - Positive affect }\end{array}$ & $\begin{array}{l}\text { - Exercise } \\
\text { - } \mathrm{BMI}\end{array}$ \\
\hline No & $\begin{array}{l}\text { - Income } \\
\text { - Wealth } \\
\text { - Age } \\
\text { - education } \\
\text { - Gender } \\
\end{array}$ & $\begin{array}{l}\text { - Friends in neighborhood } \\
\text { - Relatives in neighborhood } \\
\text { - Frequency of social meeting } \\
\text { - Marital status }\end{array}$ & -- & $\begin{array}{l}\text { - } \text { Drinking } \\
\text { - Smoking }\end{array}$ \\
\hline
\end{tabular}

Predictive SES/demographic, social, psychological and behavioral variables across race/ethnic groups were not identical (see Table 13, Chapter 6) but had several overlapping predictors. Overall, the somatic symptoms variable was consistently related to physical impairment in all race/ethnic models both in cross-sectional and longitudinal models, indicating, perhaps, the similarity of this variable with the physical impairment variable across race/ethnic groups. Respondents reporting presence of somatic symptoms had higher risk of reporting at least some level of physical impairment. Body mass index did not appear to have substantial effect in the Black and Hispanic groups because being normal weight or overweight did not change risk level in the presence of other dominant variables such as somatic symptoms and job status. In the White group, physical activity (exercise) and volunteering in charitable organizations predicted physical impairment status while these variables did not appear in the Black and Hispanic groups. Negative affect predicted physical impairment in Blacks but did not appear in models for the White and Hispanic groups. Because the negative affect variable was predictive of physical impairment in the Black group, and presence of negative affect was related to increased 
risk of reporting physical impairment in this group, race/ethnic differences in physical impairment may arise due to differences in the effect of negative affect in the Black group. Except for employment status, SES/demographic variables did not appear in models across groups, suggesting that, in general, social, psychological and behavioral variables were more strongly related to physical impairment in the direct prediction approach used in RA. Interaction effects hardly featured in the models in all groups except in one case where negative affect and somatic symptoms jointly predicted physical impairment status in Blacks. The fact that the other models did not have interaction terms may be because of using the most conservative model selection criterion, namely BIC. Fit tables of models with overlapping variables were examined to compare similar and unique risk increasing and risk reducing effects of composite IV states. The next sections briefly discuss potential effects of non-overlapping variables and effects of composite IV states of overlapping variables by race/ethnic groups for selected results.

Whites versus Hispanics: Recall that in comparing the White and Hispanic groups, exercise and volunteering in charitable organizations predicted physical impairment in the White group only, while positive affect and BMI predicted physical impairment in the Hispanic group only. Employment status and somatic symptoms predicted physical impairment in both groups. It is known that regular physical activity is related to better health status/outcome and results in this analysis suggested physical activity was more strongly related to physical impairment in Whites than it was in Hispanics potentially indicating the positive effects of physical activity. Further, volunteering in charitable organizations acts as a source of social integration factor and 
promotes well-being (Okun, August, Rook, \& Newsom, 2010). Fit analysis indicated that frequent exercise and volunteering were related to lower risk of physical impairment. Differences in relative risk of physical impairment between Whites and Hispanics, therefore, could arise due to differences in levels of engagement in behavioral (exercise) and social (volunteering) factors in the White and Hispanic groups.

Table 43: Partial fit table for unique IV states that increase risk in cross-sectional direct prediction

\begin{tabular}{|c|c|c|c|c|c|c|c|}
\hline \multirow[t]{2}{*}{ Race/ethnicity } & \multicolumn{2}{|r|}{ IV } & \multirow{2}{*}{$\begin{array}{l}\text { Data } \\
\text { freq }\end{array}$} & \multicolumn{2}{|c|}{$\begin{array}{l}\text { Model } \\
\text { calc. q(DV|IV) }\end{array}$} & \multirow[b]{2}{*}{$\mathrm{p}($ margin $)$} & \multirow[b]{2}{*}{ Ratio, $\mathrm{PI}=1$} \\
\hline & $\mathbf{J}$ & Som & & $\mathrm{PI}=0$ & $\mathrm{PI}=1$ & & \\
\hline \multirow{2}{*}{ White } & 2 & 1 & 121 & 77.1 & 22.9 & 0.084 & 1.35 \\
\hline & \multicolumn{3}{|c|}{ Marginal probability } & 83.0 & 17.0 & & \\
\hline \multirow[t]{2}{*}{ Hispanic } & $\$ 2$ & 1 & 101 & 73.1 & 26.9 & 0.041 & 1.42 \\
\hline & \multicolumn{3}{|c|}{ Marginal probability } & 81.1 & 18.9 & & \\
\hline
\end{tabular}

Note: ${ }^{\ddagger}$ unique IV states that increase risk

$\mathrm{J}=$ job status, $2=$ retired \& Som=somatic symptoms (1=present)

Analysis of composite IV states of the overlapping variables (employment status and somatic symptoms) indicated that retired Hispanics with somatic symptoms had relatively higher risk of reporting physical impairment while the corresponding composite IV states in Whites did not differ from the marginal risk to the group, if one insists on the usual 0.05 significance level (see Table 43, below). Effect sizes for the composite IV states in both groups were similar, however. Similarity of risk increasing and risk reducing composite IV states in both groups indicate that, for the matched data set in the RA method, there were no substantial differences between the White and Hispanic groups. Key dissimilarities between the groups were in the variables predictive of physical impairment status, namely the effect of physical activity and volunteering in 
charitable organizations in Whites and the effect of absence or presence of positive affect and BMI in Hispanics which may be related to differences in physical impairment status.

Whites versus Blacks: Predictive variables in the White and Black groups were also not identical. In the White group, unique predictive variables were exercise, positive affect and volunteering in charitable organizations while negative affect predicted physical impairment in the Black group only. Common predictors of physical impairment in the White and Black groups were employment status, BMI and somatic symptoms. Race/ethnic differences in physical impairment status may result from differences in the predictive variables. Similar to results in models comparing Whites with Hispanics, two health promoting variables (exercise and volunteering in charitable organization) predicted physical impairment. Use of the term health promoting here is justified by the fact that participating in volunteer activities as well as engaging at least in moderate physical exercise are known to provide better health benefits.

Fit analysis of models with only the overlapping variables showed that being employed, having normal weight and absence of somatic symptoms in Whites, and being employed, being overweight and presence of somatic symptoms in Blacks, reduced risk relative to each group's marginal risk (Table 44, upper portion). The unique protective effects in the White group makes sense because being employed, having normal body weight and absence of somatic symptoms are desirable states. The corresponding effect size in Blacks was similar but not significant at the 0.05 level, perhaps due to low sample size. However, the unique protective IV states in the Black group, that is, being 
employed, being overweight, and presence of somatic symptoms, appears to be counterintuitive.

Table 44: Partial fit tables for unique IV states that increase/decrease risk in cross-sectional direct prediction

\begin{tabular}{|c|c|c|c|c|c|c|c|c|}
\hline \multicolumn{9}{|c|}{ White versus Black comparison (Model was JPI:BmiPI:SomPI) } \\
\hline \multirow{2}{*}{$\begin{array}{l}\text { Race/ } \\
\text { ethnicity }\end{array}$} & \multicolumn{3}{|c|}{ IV } & \multirow{2}{*}{$\begin{array}{c}\text { Data } \\
\text { Freq. }\end{array}$} & \multicolumn{2}{|c|}{$\begin{array}{c}\text { Model } \\
\text { calc. } \mathrm{q}(\mathrm{DV} \mid \mathrm{IV})\end{array}$} & \multirow[b]{2}{*}{$\mathrm{p}($ margin $)$} & \multirow[b]{2}{*}{ Ratio, $\mathrm{PI}=1$} \\
\hline & $\mathbf{J}$ & Bmi & Som. & & $\mathrm{PI}=0$ & $\mathrm{PI}=1$ & & \\
\hline \multirow{3}{*}{ White } & $\lambda 1$ & 1 & 0 & 41 & 96.7 & 3.3 & 0.017 & 0.19 \\
\hline & 1 & 2 & 1 & 45 & 83.0 & 17.0 & 0.902 & 0.96 \\
\hline & \multicolumn{4}{|c|}{ Marginal probability } & 82.3 & 17.7 & & \\
\hline \multirow{3}{*}{ Black } & 1 & 1 & 0 & 17 & 97.4 & 2.6 & 0.074 & 0.13 \\
\hline & $\lambda_{1}$ & 2 & 1 & 56 & 90.6 & 9.4 & 0.05 & 0.47 \\
\hline & \multicolumn{4}{|c|}{ Marginal probability } & 80.1 & 19.9 & & \\
\hline \multicolumn{9}{|c|}{ Black versus Hispanic comparison (Model was JPI:BmiPI:SomPI) } \\
\hline & $\mathrm{J}$ & $\mathrm{Bmi}$ & Som. & Freq. & $\mathrm{PI}=0$ & $\mathrm{PI}=1$ & $\mathrm{p}($ margin $)$ & Ratio, $\mathrm{PI}=1$ \\
\hline \multirow{4}{*}{ Black } & 2 & 1 & 0 & 30 & 90.9 & 9.1 & 0.073 & 0.40 \\
\hline & 2 & 2 & 1 & 106 & 71.6 & 28.4 & 0.175 & 1.24 \\
\hline & $\$ 3$ & 1 & 1 & 17 & 55.1 & 44.9 & $\mathbf{0 . 0 3 0}$ & 1.96 \\
\hline & \multicolumn{4}{|c|}{ Marginal probability } & 77.1 & 22.9 & & \\
\hline \multirow{4}{*}{ Hispanic } & $\lambda 2$ & 1 & 0 & 28 & 95.7 & 4.3 & 0.049 & 0.23 \\
\hline & $\$ 2$ & 2 & 1 & 80 & 72.1 & 27.9 & 0.039 & 1.48 \\
\hline & 3 & 1 & 1 & 15 & 62.0 & 38.0 & 0.059 & 2.01 \\
\hline & \multicolumn{4}{|c|}{ Marginal probability } & 81.1 & 18.9 & & \\
\hline
\end{tabular}

Note: ${ }^{\lambda}$ unique IV states that reduce risk; ${ }^{\star}$ Unique IV states that increase risk.

For $\mathbf{J}=$ Job status, (1=employed, $2=$ retired, 3=other), Bmi (1=normal, 2=overweight/obese) \& Som=somatic symptoms, $(1=$ present, $0=$ absent $)$

How is it that being overweight and having somatic symptoms not related to increased risk of physical impairment? Several factors may account for this outcome. First, being overweight or obese may be an inadequate measure of well-being in the Black group as indicated in other studies (Flegal et al., 2012) and, therefore, may not pose significant risk. Second, being employed may be more beneficial to Blacks that mitigates the effects of somatic symptoms. 
Blacks versus Hispanics: In Black versus Hispanic comparison, the extra predictor in the model for Blacks was negative affect while in the model for Hispanics it was positive affect. The common predictive variables were employment status, BMI, and somatic symptoms. Notice these common predictors were identical to the common predictors in White versus Black comparison above. Any differences in physical impairment status between Blacks and Hispanics may be due to these unique predictors in each group. However, fit analysis of models with only the overlapping predictors indicated a few unique composite IV states in Blacks and Hispanics that increased risk of physical impairment relative to the marginal risk in each group (Table 44, lower portion).

Among Blacks, falling in job category 'other' $(\mathrm{J}=3)$, having normal BMI and presence of somatic symptoms increased risk. The corresponding IV states in the Hispanic group posed similar risk (i.e., had similar effect sizes) although in the Hispanic group, risk of physical impairment was not significantly different from the marginal risk to the group at 0.05 significance level. The important factor in the model for Blacks appeared to be falling in job category 'other' because in White versus Black comparison above, being employed, even in the presence of somatic symptoms and being overweight $(\mathrm{Bmi}=2)$, was protective in Blacks. In other words, being normal weight $(\mathrm{Bmi}=1)$ in the presence of somatic symptoms ( $\mathrm{Som}=1)$ and being retired $(\mathrm{J}=2)$ or falling in 'other' job category $(\mathrm{J}=3)$ was not protective in the Black group further indicating race/ethnic differences in the effects of BMI on risk of physical impairment. In the Hispanic group, the risk for respondents with these IV states was similar to the marginal risk. Unique composite IV states in the Hispanic group that increased risk was being retired $(\mathrm{J}=2)$, 
being overweight $(\mathrm{Bmi}=2)$ and presence of somatic symptoms $(\mathrm{Som}=1)$. This does not appear to be counterintuitive. However, it suggests that being retired in Hispanics may have different effects on physical impairment than its effects on physical impairment in the Black and White groups. Risk of physical impairment for retired Blacks who were overweight and had somatic symptoms was not different from the marginal risk to the group. The effect sizes of the composite IV states were substantially different between these groups. This may be because being overweight in Blacks did not pose significant risk, and retirement together with high BMI, had different effect on physical impairment in the Hispanic group.

Summary: As the above discussion indicates, some differences between race/ethnic groups of composite IV states that increased or decreased risk given fit tables of overlapping variables were noted. For instance, being overweight or obese may have higher adverse effects in Whites than in Blacks, while being employed in the presence of somatic symptoms may mitigate risk of physical impairment in Blacks more than it does in Whites. Most of the race/ethnic differences, therefore, can be attributed to differences in the non-overlapping predictive variables, as discussed above.

Longitudinal models: Longitudinal models, as stated earlier, had fewer variables predictive of physical impairment (i.e., they were less complex. See Table 22, Chapter 6) and also had fewer overlapping variables. Employment status in the cross-sectional models appeared in all models while in the longitudinal models, it appeared only in one model. In the cross-sectional models, BMI appeared more frequently than in the 
longitudinal models and volunteering appeared only in the White group in the crosssectional models, whereas, it also appeared in the model for Blacks in the longitudinal models (see Table 13, Chapter 6 for side by side comparison).

In models using longitudinal data, exercise, at the second time point, predicted physical impairment at the third time point in Whites while volunteering in organizations predicted physical impairment at the third time point in both Whites and Blacks. White respondents who engaged in some exercise and volunteered in organizations had lower risk of reporting physical impairment irrespective of BMI status. Negative affect predicted physical impairment in Blacks and Hispanics but not in Whites. Blacks and Hispanics reporting negative affect and somatic symptoms had increased risk of physical impairment. These two psychological variables are closely related and form part of the Center for Epidemiologic Studies Depression (CES-D) scale used in estimating depressive tendencies in populations. Race/ethnic variations in risk of physical impairment likely arise due to differences in the predictive variables. Fit analysis of composite IV states that increase or reduce risk for models with overlapping variables are discussed below.

Whites versus Hispanics: In comparing White versus Hispanic models, all predictive variables were unique, that is, exercise, BMI and volunteering predicted physical impairment in Whites, whereas somatic symptoms and negative affect predicted physical impairment in Hispanics. Clearly, corresponding IV states cannot be compared and differences in risk of physical impairment for Whites and Hispanics may be 
attributable to these differences in the predictive variables. It is important to note that absence of somatic symptoms and negative affect in Hispanics, and at least some exercise together with having normal BMI and volunteering in organizations in Whites appear to decrease risk of physical impairment.

Whites versus Blacks: Unique predictive variable in Whites was physical activity (exercise), while in Blacks, negative affect and employment status were unique predictors of physical impairment. Volunteering in organizations and exercise that appeared in the longitudinal model for Whites also appeared in the cross-sectional model for the White group, indicating reliability of the relationships between these variables and physical impairment for this group. Part of the White-Black difference in risk of physical impairment, therefore, may be attributed to the different predictive variables.

The overlapping variables were somatic symptoms and volunteering in charitable organizations. In both groups, absence of somatic symptoms and engaging in volunteering activities reduced risk while presence of somatic symptoms and not engaging in volunteering increased risk relative to the marginal risk. In this regard, between-group differences of the effects of somatic symptoms and volunteering in charitable organizations on physical impairment status were minimal. Although volunteering is known to be related to better health outcomes because it provides opportunities that promote social integration as well as purposeful living which may be related to psychological well-being, it is possible that only healthier respondents engage in volunteering activities. These outcomes were not as might be expected. However, 
unique protective composite IV states in the Black group were absence of somatic symptoms and absence of volunteering in organization (Table 45). This raises the question as to why Whites without somatic symptoms who did not volunteer in charitable organizations had similar risk to the marginal risk to the group. It is likely that volunteering, on average, provides different social benefits to Whites and Blacks where the beneficial effects are greater for Whites. Similarly, absence of somatic symptoms may be a more important protective factor in the Black group than engaging in volunteering activities. This is not to say that volunteering is harmful to Blacks, rather absence of somatic symptoms in Blacks appears to be more protective than the protective effects of volunteering, effectively providing a sort of ranking of variables that are important by race/ethnic groups.

Table 45: Partial fit table for unique IV states that decrease risk in longitudinal non-path models

\begin{tabular}{|c|c|c|c|c|c|c|c|}
\hline Race/ethnicity & \multicolumn{2}{|c|}{ IV } & Data & \multicolumn{2}{|c|}{$\begin{array}{c}\text { Model } \\
\text { calc. } \mathrm{q}(\mathrm{DV} \mid \mathrm{IV})\end{array}$} & & \\
\hline & Som. & Vo & Freq. & $\mathrm{PI}=0$ & $\mathrm{PI}=1$ & $\mathrm{p}($ margin $)$ & Ratios, PI=1 \\
\hline \multirow{2}{*}{ White } & 0 & 0 & 220 & 83.6 & 16.4 & 0.142 & 0.80 \\
\hline & \multicolumn{3}{|c|}{ Marginal prob. } & 79.6 & 20.4 & & \\
\hline \multirow{2}{*}{ Black } & ${ }^{\lambda} 0$ & 0 & 154 & 88.6 & 11.4 & 0.010 & 0.58 \\
\hline & \multicolumn{3}{|c|}{ Marginal prob. } & 80.3 & 19.7 & & \\
\hline
\end{tabular}

Note: ${ }^{\lambda}$ unique protective IV states; Vo=volunteering $(0=$ no $)$

Blacks versus Hispanics: In the longitudinal model for the Hispanic group, only somatic symptoms and negative affect predicted physical impairment, whereas in Blacks, somatic symptoms, negative affect, BMI and volunteering in organizations predicted physical impairment. All predictors in the Hispanic group appeared in the model for Blacks but the model for Blacks had additional predictors. Thus, differences in physical impairment status between the Black group and the Hispanic group may be due to the 
extra predictors in the model for Blacks. Analyzing fit table for the common predictors, that is, for models containing only somatic symptoms and negative affect, revealed that there were no unique composite IV states that increased or decreased risk in either group. In both groups, similar composite IV states increased risk and decreased risk. This indicates that there were not substantial between-group differences given the overlapping variables and any differences between the two groups may be due to different predictive variables.

Summary: To summarize, the cross-sectional models were more complex than the longitudinal models. Complexity does not necessarily depend on number of predictors but in the present study, models with more variables were more complex because most variables had similar bins (categories). In most cases, composite IV states for the overlapping variables that increased or decreased risk were similar between any groups compared, suggesting that any differences in physical impairment by race/ethnicity may be attributed to the different predictive variables. Some composite IV states that reduced risk in Blacks but not in Whites, such as the absence of somatic symptoms and not volunteering in charitable organizations, were counterintuitive. Such results likely point to the utility of volunteering in Whites and the importance of absence of somatic symptoms in Blacks regardless of volunteering status. Being overweight appeared to be more consequential in the White group than in the Black group consistent with previous studies that suggested being overweight may not have similar adverse effects in Blacks (Flegal et al., 2012). Similarly, beneficial aspects of behavioral factors including exercise and social factors (volunteering in charitable organizations) were more frequently related 
to physical impairment status in Whites indicating the protective effects of these variables in this group.

\subsection{Cross-Sectional and Longitudinal Path Analysis of Physical Impairment Status}

This section discusses selected results of indirect association of SES/demographic variables with physical impairment through social, psychological, and behavioral factors. In cross-sectional and longitudinal path models, the effects of the selected SES/demographic factors on the selected intermediate variables (i.e., on $\mathrm{S}, \mathrm{P}$ and $\mathrm{B}$ ) were mostly either moderate or strong because only SES/demographic variables strongly associated with intermediate variables were first selected using neutral system search. In both cross-sectional and longitudinal path models, alcohol consumption levels (drinking) and smoking were the only behavioral variables that appeared across groups. However, while the effects of the selected variables on alcohol consumption and smoking were mostly moderate or strong, the adjusted effects of drinking or smoking on physical impairment were nearly zero.

Cross-sectional path models: Similar to direct prediction analyses described in the previous section, cross-sectional path models suggested stronger relationships compared to longitudinal path models. In cross-sectional models, psychological variables featured more prominently while only one social variable was related to SES/demographic variables. 
Table 46: SES/demographic, social, psychological and behavioral variables in cross-sectional path models for the physical impairment outcome variable

\begin{tabular}{|c|c|c|c|c|}
\hline \multirow{2}{*}{$\begin{array}{l}\text { Variable } \\
\text { in path } \\
\text { model? }\end{array}$} & \multirow{2}{*}{$\begin{array}{c}\text { SES } \\
\begin{array}{c}\text { Demographic } \\
\text { variables }\end{array}\end{array}$} & \multicolumn{3}{|c|}{ Intermediate $\{\mathrm{SPB}\}$ variables } \\
\hline & & Social & Psychological & Behavioral \\
\hline Yes & $\begin{array}{l}\text { - Wealth } \\
\text { - Income } \\
\text { - Gender } \\
\text { - Job } \\
\text { - Education }\end{array}$ & - Marital status & $\begin{array}{l}\text { - Negative affect } \\
\text { - Somatic symptoms }\end{array}$ & 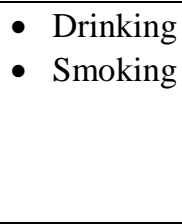 \\
\hline No & - Age & $\begin{array}{l}\text { - Friends in neighborhood } \\
\text { - Relatives in } \\
\text { neighborhood } \\
\text { - Volunteering }\end{array}$ & - Positive affect & $\begin{array}{l}\text { - Exercise } \\
\text { - } \text { BMI }\end{array}$ \\
\hline
\end{tabular}

Whites versus Hispanics: In White versus Hispanic comparison, gender and income jointly predicted marital status but the adjusted effect of marital status on physical impairment in the Hispanic group was slightly higher. The main point here was that in both groups, gender and income were associated with marital status indicating that the structural relationships of these variables for the matched White-Hispanic group were similar. Further, wealth predicted negative affect in both Whites and Hispanics, and the adjusted effects of negative affect on physical impairment in both groups were moderate. As in the previously discussed findings, these results suggest similarity of the structure and strength of relationships of these variables for the two race/ethnic groups where an indirect relationship may exist as hypothesized. Although indirect relationships, based on the path strength through the psychological variable, are possible, differences in path magnitude do not appear to be substantial between Whites and Hispanics. The SEM results indicated that SES was indirectly related to physical impairment through the latent psychological variable composed of somatic symptoms, positive affect and negative affect pointing to similar relationships of these variables in both RA and SEM methods. 
Whites versus Blacks: Comparing White and Black path models with psychological variables as an intermediate variable, wealth in Whites was related to somatic symptoms while education level and employment status were jointly related to somatic symptoms in Blacks. Note the different SES variables related to the same psychological variable in each group. These different relationships may indicate the protective effects of education and job status on somatic symptoms for Blacks and the protective effects of wealth in Whites. In other words, although higher education and being employed may be beneficial in Whites, wealth was a stronger predictor of somatic symptoms in Whites. Further, the adjusted effect of somatic symptoms on physical impairment in the White group was strong while the effect was moderate in the Black group.

Overall, there was possible indirect relationship between the respective predictive SES variables and physical impairment in each group. The implication is that wealth reduced somatic symptoms in Whites and absence of somatic symptoms was related to lower risk of physical impairment, and in Blacks, higher levels of education and being employed were related to absence of somatic symptoms and, absence of somatic symptoms, in turn, reduced risk of physical impairment based on the relationships examined in non-path models in previous section. A useful point in this discussion is that the somatic symptoms to physical impairment relationships in Whites and Blacks were substantial. Differences in physical impairment status may arise due to differences in the predictive value of the selected SES variables, but earlier analysis indicated similar composite IV states in Whites and Blacks likely pose similar risk in the presence of 
somatic symptoms, pointing to differences in physical impairment by race/ethnic groups to be due to differences in how the SES variables related to physical impairment.

Blacks versus Hispanics: Employment status predicted somatic symptoms in Blacks, while, wealth predicted negative affect in Hispanics. Specific SES variables were related to different psychological variables in the groups. The adjusted effects of somatic symptoms on physical impairment in the Black group as well as the adjusted effects of negative affect on physical impairment in the Hispanic group were moderate suggesting indirect relationships. Some differences that may be observed in physical impairment status, therefore, may be attributable to differences in how specific SES variables relate to physical impairment through different psychological factors.

Summary: In summary, path values from SES to a selected psychological variable and from the selected psychological variable to physical impairment status were strong across groups. Note that positive affect did not appear in the cross-sectional path models. Alcohol consumption (drinking) and smoking were related to SES/demographic variables but the adjusted path values from smoking and drinking to physical impairment were nearly zero in all groups. These results suggest that there does not appear to be appreciable between group differences in structural relationships of the intermediate variables and physical impairment status across groups.

Longitudinal path models: In the previous section, longitudinal models for the direct prediction of health outcome variables were weak with fewer predictive variables and smaller uncertainty reduction in the dependent variables. In longitudinal-path models 
SES/demographic factors appeared to be moderately or strongly related to physical impairment through psychological variables in the Black group but not in the White. Indirect longitudinal paths from SES/demographic factors to physical impairment through social, psychological or behavioral variables were not strong in the Hispanic group. Only a few models had intermediate to physical impairment paths with values greater than 3.5\% uncertainty reduction in physical impairment status. The structure of the relationships between variables and across groups in the longitudinal path models were similar to the cross-sectional path results but relationships were weaker in the longitudinal models

\section{Overnight Hospitalization}

Unlike models for the physical impairment outcome variable, according to BIC model selection criterion, SES/demographic, social, psychological and behavioral factors barely predicted overnight hospitalization variable directly or indirectly through the selected intermediate ( $\{$ SPB $\}$ ) variables. These results suggest that SES/demographic, social psychological and behavioral variables may not be the best variables that one needs to use to predict overnight hospitalization and compare by race/ethnicity given the data sets. Nonetheless, the sections below briefly discuss some of the weak relationships observed in models that suggested predictive variables.

\subsection{Cross-Sectional and Longitudinal Analysis of Overnight Hospitalization}

In the White versus Hispanic group comparison, gender and negative affect independently predicted overnight hospitalization in Hispanics while the BIC model for 
the White groups suggested no predictive variables. Percent uncertainty reduction $(4.3 \%)$ in overnight hospitalization variable in the Hispanic group was small, however. Thus, between-group variation may result from variations of the effects of gender and negative affect on hospitalization in the Hispanic group. Analysis of the fit table for the Hispanic model indicated that males without negative affect had hospitalization risk similar to the marginal risk to the group, whereas, female Hispanics without negative affect had significantly lower hospitalization risk relative to the marginal risk to the group. Similarly, overnight hospitalization for Hispanic males with negative affect increased while the risk for their female counterparts was significantly lower than the marginal risk to the group. These were interesting results which were not part of any hypotheses motivating this study but raise questions that may be studied further later.

Comparing the models for White with the models for Blacks, the best BIC model was the independence model, indicating lack of variables predictive of overnight hospitalization in both groups. In the Black versus Hispanic comparison, employment status was related to overnight hospitalization in Blacks, but in Hispanics, gender and negative affect predicted overnight hospitalization. For Hispanics, the same model appeared when comparing Whites with Blacks. Effects of composite IV states in the Hispanic group were as above. Effects of various states of employment status in Blacks were not counterintuitive. Employed Blacks had lower risk of hospitalization while Blacks falling in job category 'other' were at higher risk of hospitalization relative to the marginal risk. Retired Blacks had similar risk to the marginal risk to the group. There was nothing extraordinary about these results and one could safely conclude this trend would 
be the similar in other race/ethnic groups for different levels of employment status. The notable point is the process by which employment status in Blacks may reduce or increase risk compared to other races. Slight variations are likely to arise due to these variations in predictive variables in Blacks and Hispanics.

Results for longitudinal models, once again, indicated that SES/demographic, social, psychological, and behavioral factors were not related to overnight hospitalization. Only somatic symptoms at the second time point predicted overnight hospitalization at the third time point in the White group, and only physical activity at the second time point was related to overnight hospitalization at the third time point in the Black group. In the Hispanic group, the best model was the independence model indicating no relationships. Absence of somatic symptoms had similar risk to the marginal risk to the group while presence of somatic symptoms increased risk of overnight hospitalization. Thus, in Whites presence of somatic symptoms was related to higher risk of overnight hospitalization and physical impairment as indicated in previous sections. While exercise was related to physical impairment in the White group, here, exercise was related to overnight hospitalization in the Black group. Risk of overnight hospitalization gradually decreases with various levels of physical activity. Fit results indicated that Blacks who did not engage in any physical activity had significantly higher risk of overnight hospitalization relative to the margin while those who engaged in frequent physical activity had significantly lower risk of hospitalization. Blacks who engaged in some physical activity had similar risk of hospitalization as the marginal risk to the group. These findings may not be surprising but it is essential to note how levels of exercise 
relate to risk levels of hospitalization in Blacks, whereas, this variable does not show up in models for other groups.

These weak relationships of SES/demographic, social, psychological, and behavioral factors with overnight hospitalization mirror the SEM results, where the path coefficients for the relationship from SES to overnight hospitalization, as well as social, psychological, and behavioral factors to overnight hospitalization were weak.

\subsection{Cross-Sectional and Longitudinal Path Analysis of Overnight Hospitalization}

In RA path analyses of the overnight hospitalization outcome variable, the strength of associations between SES and the mediators and, in turn, between the mediator and overnight hospitalization were weaker than when the outcome variable was physical impairment. This weak relationships of SES with social, psychological and behavioral variables, and in turn, the weak relationship of social, psychological and behavioral variables with overnight hospitalization reflect the weak relationships found in non-path models as discussed in section 8.3.

Associations between SES variables and the intermediate variables (social, psychological and behavioral factors) were similar in terms of variables selected and uncertainty reductions in the intermediate variables but the intermediate ( $\{\mathbf{S P B}\})$ to overnight hospitalization path values were nearly zero. These were in contrast to the path models explored with physical impairment as the outcome variable. 
In summary, results for the overnight hospitalization variable indicated that SES/demographic, social, psychological, and behavioral variables were not related to overnight hospitalization. Older adults (age $>65$ ) are covered by Medicare and in some cases by both Medicaid and Medicare, thus, barriers to accessing medical care are theoretically reduced in this group. As far as the hypotheses motivating this study, it appears that SES/demographic and $\{\mathbf{S P B}\}$ variables were not related to overnight hospitalization across groups, an indication that these variables may not be optimal in predicting overnight hospitalization. 


\section{CHAPTER 9.0: CONTRIBUTIONS, LIMITATIONS AND FUTURE STUDY}

\subsection{Comparing SEM and RA Results}

It is important to recall that data were matched on SES/demographic variables for the RA method because model selection depended on $\mathrm{N}$; further, in the OCCAM implementation RA, complex sampling design adjustments for the HRS data were not used. The social integration variable was an index and the psychological variable was a latent variable in the SEM analysis but disaggregated social and psychological variables were used in RA. Therefore, only general comparisons between results from the two methods can be made. Overall, SEM and RA results indicated some similar trends. First, both methods indicated that SES, social, psychological and behavioral variables were weakly related to overnight hospitalization. Second, behavioral factors were weakly related to physical impairment status in the path model results in RA as well as in the SEM results.

\subsection{Policy Implications}

The sample in this study consisted of older Americans. Several common factors characterize individuals as they age irrespective of race or ethnicity. Overall, aging (post retirement) is, on average, related to growing poor due to loss of income from jobs or increased expenses on medical care. With age, onset of diseases (multiple chronic conditions in many cases) begins and decline in carrying out activities of daily living accelerates. Thus, with age, racially and ethnically diverse individuals likely become more similar in their health status. 
Income, wealth, education, social integration, psychological status, and behavioral factors have the potential to accelerate or slow down physical impairment, delay onset of diseases and reduce overnight hospitalizations thereby improving quality of life. Because SES is associated with race/ethnicity where Whites fall in higher SES category, it is believed that minorities experience worse health outcomes compared to Whites. But it is also known that differences in health status by race/ethnicity narrow with age. A common factor that potentially contributes to psychological stressors among race/ethnic groups transitioning to retirement is uncertainty about several aspects of living conditions including financial and social arrangements. Lower SES individuals are particularly susceptible to adverse effects of uncertainty at this stage. Additional stressors for minorities are potential discriminative practices entrenched in organizational and social cultures. Policies need to be cognizant of the role that these variables play across one's life course when attempting to address the well-being of individuals among racially/ethnically diverse populations.

Results in this study indicate effects of SES on social, psychological and behavioral factors, and, in turn, effects of these factors on health outcome variables, and that these relationships were similar across groups. Higher SES was related to lower physical impairment through lower depressive symptoms scores and higher social integration but effect sizes were small albeit significant in some paths (mainly in path models for the White group). Therefore, regarding older population, policies may have to look for levers that alleviate poverty, increase access to preventive care, and increase inclusive communal activities for the elderly. Policies and programs regarding healthy 
aging are beneficial, but more importantly, policies that take a life course view of an individual's existence and promote healthy habits from early on, improve living conditions through education, income, and social networks may be more useful in addressing observed disparities in health.

\subsection{Contributions of the Present Study to the Literature}

Prior research has established SES and race/ethnicity based disparities in health outcomes. Longitudinal studies of potential mediators including social, psychosocial and behavioral variables are limited, however. Some studies (Krause \& Borawski-Clark, 1995; Turner \& Marino, 1994; Vonneilich et al., 2012) investigated the relationships between socioeconomic status, social relationships and health status. These studies, however, used racially homogeneous sample and only sought to investigate SES based disparities without extensive examination of possible intermediate variables between SES and health variables. Although the role of social, psychological, and behavioral factors as potential mediators have been proposed, these pathways have not been extensively studied longitudinally. The present study has addressed this gap and has made several contributions to the disparities literature as noted below.

The first part of the current research examined whether SES was indirectly related to physical impairment and overnight hospitalization through social, psychological and behavioral factors, and whether these relationships varied by race/ethnicity. The study supported that there was a consistency in correlational relationships of most of the hypothesized pathways. Specifically, results in the present study supported that income, 
wealth, education level, and employment were positively related to social integration, and in turn, social integration (higher scores indicate better integration) was inversely related to physical impairment scores. This is an important empirical support of the conceptual model motivating this study. Wealth and income significantly predicted physical impairment status through the social integration variable in Whites. Although signs of the path coefficients were consistent with the hypothesis among Blacks and Hispanics, indirect effects were not significant. These support the role of social integration in linking SES and physical impairment across groups

The path model with the psychological variable as a mediator showed that all four SES variables were negatively related to depressive symptoms. In turn, the depressive symptoms variable was positively related to physical impairment consistent with the hypothesis. Once more, these results were in concordance with the hypothesized relationships between SES and psychological factors, and between psychological factors and physical impairment status. Although indirect effects were significant in the White group, effect sizes were similar across groups.

Regarding behavioral variables as intermediate variables, results of this study showed that the indirect effects of SES on physical impairment through BMI were consistent with the hypothesis. However, higher SES was related to higher consumption of alcoholic beverages, contrary to hypothesized relationships. Therefore, in a national scale study, alcoholic beverage consumption may not reflect problem drinking, rather, alcohol consumption may instead indicate higher SES. 
Where results failed to support hypothesized relationships was in the indirect association of SES with overnight hospitalization through the social, psychological, and behavioral variables. Two observations regarding this outcome need to be stated. First is that the lag between adjacent waves was two years for the overnight hospitalization outcome variable which may have not been optimal. Second, the proposed intermediate variables may not be ideal predictors of this outcome variable because social, psychological, and behavioral variables may only affect other variables that are more proximally related to overnight hospitalization. Follow up analysis using chi-square test of nested models for significant indirect relationships suggested paths were equal across race/ethnic groups.

The foregoing discussion illustrated the unique contributions of this study to the disparities literature by supporting the hypothesized relationships between SES, intermediate variables ( $\{$ SPB $\})$, and health outcome variables (physical impairment and overnight hospitalization). Although results indicated significant indirect effects in some groups, follow up analysis, indicated that paths were not different across groups.

The second part of this dissertation, using RA, a heuristic search method based on information and graph theories, explored SES/demographic, social, psychological, and behavioral variables that predicted physical health status and overnight hospitalization. This approach, simultaneously, selected the best predictors including interaction effects and provided uncertainty reductions in the dependent variable (DV) due to the selected predictive independent variables (IVs). Selected models were further examined using fit 
analysis to identify and highlight IV states that increased or decreased risk of physical impairment or overnight hospitalization between race and ethnic groups for overlapping predictors. Because of the exploratory nature of RA, another beneficial aspect of the approach was that it generated hypotheses that may be further investigated in the future. Using this approach, the present study showed that SES/demographic, social, psychological and behavioral variables independently predicted physical impairment. Physical activity (exercise) and volunteering in organizations appeared more frequently in the White group showing that Whites engaged in health promoting behavioral and social activities more than Blacks or Hispanics. Analysis of the matched data also revealed that social, psychological, and behavioral variables were more predictive of physical impairment than SES/demographic variables except employment status, which appeared to predict physical impairment in the cross-sectional models. Mostly, composite IV states that increased or reduced risk, in any two groups compared, were similar. That is, identical IV states increased risk in Whites the same way those IV states increased risk in Hispanics and Blacks for the overlapping variables. In some cases, however, IV states that increased or decreased risk differed by race/ethnicity. Most race/ethnicity based differences may be due to variability of predictive IVs in each race/ethnic group.

Socioeconomic/demographic variables, social, psychological and behavioral factors had minimal effect on overnight hospitalization. Thus, either the time lag was sub-optimal or the variables were not ideal in predicting overnight hospitalization. This result may inform the structure of future studies. 
Further, the RA approach explored associations between SES/demographic and the mediating variables ( $\{\mathbf{S P B}\})$, and in turn, between the mediating variables and health variables. The selected SES/demographic variables were strongly or moderately related to intermediate variables, but the intermediate variables were not strongly or moderately related to physical impairment except for the psychological variables (somatic symptoms, positive affect and negative affect). Recall that the data for RA method were matched on SES/demographic variables. Few paths were identical across race/ethnic groups and path values were similar across groups. There were differences in the SES/demographic variables that were associated with intermediate variables, and race/ethnic differences in physical impairment, therefore, could be due to differences in predictive variables. Again, none of the SES/demographic factors appeared to be indirectly related to overnight hospitalization.

Overall, the SEM and RA results supported hypothesized relationships across race/ethnic groups. In non-significant relationships, correlational relationships were consistent with the hypotheses. Results from both methods pointed to poor indirect relationship between SES/demographic factors and overnight hospitalization. One outcome contrary to the hypothesis was the positive relationship between SES and alcohol consumption levels.

\subsection{Advantages and Disadvantages of the Two Analytic Approaches}

This study used two distinct analytic approaches. As with any method, each has limitations and strengths. Some of the advantages of RA included detection of non-linear 
relationships, identification of interaction effects not specified a priori, and selection of variables (features) and computation of uncertainty reductions using only the selected predictive variables. RA, implemented in OCCAM, was used here to conduct a neutral search to detect association between equally ranking variables (i.e., without specifying IV/DV), and to use suggested relationships in a directed search either in exploratory mode where predictive variables are selected and uncertainty reductions are calculated, or in a directed confirmatory mode where uncertainty reduction in a DV due to specified IVs is computed. Selected models were analyzed by fitting the model to the data. This explicitly calculates risk of composite IV states relative to the margins and provides information on IV states that elevate or reduce risk of reporting physical impairment and overnight hospitalization.

The disadvantage in the RA path analysis was inability to assign a net indirect effect quantifying the effect of selected SES/demographic factors on physical impairment (or overnight hospitalization) through the selected intermediate ( $\{\mathbf{S P B}\})$ variables. The RA approach also required that quantitative variables be binned; a step that may lead to loss of information although this loss of information can be offset in that RA method detects non-linear relationships. Because model selection was dependent on the sample size, the OCCAM implementation of RA also, ideally, required equal samples which reduced the sample sizes for the White and Black groups when matching with the Hispanic group to obtain comparable sample sizes for group comparison. 
An advantage of SEM was the clear interpretation of path coefficients; using continuous variables thus retaining information, and computing path coefficients easily by incorporating several regression equations at once. The HRS uses a complex sampling design and this study adjusted for design specifications in the SEM framework by including a weight variable, as well as stratification and clustering variables. SEM also allows for latent variable modeling in path analysis or growth curve modeling. Thus, SEM is a flexible framework for studying cross-lagged models, growth curve models, inter-group and intra-group variations in trajectories and performing multiple group path equality tests. A disadvantage of SEM and regression in general is that one is forced to pre-specify relationships to fit and may miss non-linear interactions.

Feedback effects were not examined in this study and both analytic approaches do not provide easy implementation of examination of such relationships. Nevertheless, the above analytic approaches can be viewed as complementary in that the objective of both methods was to discover relationships, quantify parameters and compare predictive variables across groups.

\subsection{Limitations and Future Study}

\section{Limitations}

Although this study contributes to race/ethnic health disparities literature, as outlined above (section 9.3), it has several limitations. It used secondary data and relied on measures not specifically designed for the present hypotheses. Thus, several of the measures used here, at best, are crude approximations of the variables of interest in these 
hypotheses. It is plausible that, for an individual with average social integration scores, the benefits of social integration may canceled out by other social conflicts, such as spousal conflicts, conflicts with other relatives or friends that are not accounted for in this study. Asking respondents whether relatives/friends live in the neighborhood assume there will be positive interaction, but this may not necessarily be true. Follow up questions about activities and frequency of engaging in these activities with friends and relatives would have allowed a better approximation to social integration. Further, to better capture level of social integration, information about respondent's engagement with friends at the work place (for those gainfully employed) including group activities unrelated to work would have been desirable. The physical activity (exercise) questions were not consistent across waves apart from being too general and BMI is a narrow indicator of behavioral factors. A better measure of physical activity may be questions targeting purposeful exercise rather than questions that allow or encourage inclusion of other strenuous activity related to work.

The data used in this study were self-reported population data collected over time. Inherent in longitudinal self-reported surveys are issues of missing data due to attrition and skipping of questions. Missing data in longitudinal studies are likely to bias results downward. Variations between groups may be reduced because the data came from older population that is subject to selection problems through various mechanisms such as death or survival of groups with similar SES/demographics, social, psychological and behavioral characteristics. Whites, Blacks, and Hispanics who live to be older may have similar characteristics because inter-group differences are known to diminish with age. 
Further, the data remain subjective and issues with subjective reports, including inaccurate or exaggerated responses can affect measurement precision.

A large proportion of Hispanic respondents in the HRS were born outside the United States. Respondents born and raised outside the US may have different cultural orientation than their native born counterparts. Attitudes, behaviors and living arrangements of racial and ethnic groups vary. Although cultural practices tend to become similar over time, some variations between native born and foreign born respondents are inevitable, necessitating caution in making unqualified generalization of these findings to the rest of the population.

Some of the SES variables including income and wealth were absolute values that did not take into account household size. Thus, although one may have higher income, relative to household size the reported income may suggest conclusions that are unreliable. Conversely, for a single respondent household, some fraction of medium income may be sufficient. Appropriate weighting of the SES variables relative to individuals in the household supported may provide a better measure of the true differences in SES by race/ethnicity.

Finally, this study compared predictive paths across groups rather than estimate the effects of race or ethnicity on health status or health outcomes controlling for the effects of SES variables. Estimation of direct effects of race/ethnicity, however, is another alternative approach that is commonly used. 


\section{Future Study}

Future studies need to address the limitations highlighted above. First, it will be valuable to use similar approaches to examine pathways using data for populations beginning at younger age. This can be accomplished by studying several cohorts beginning at younger ages or by longer follow up of study respondents beginning before the age of 51, rather than at age 51 which was a cut-off point for eligibility in the HRS study. Nativity may play a role in SES status, psychosocial status and behavioral characteristics, thus, prior to group comparison, establishing that Hispanics of different origins have similar characteristics will be useful.

Taking into account family size, particularly dependent family members or number of relatives getting economic assistance from the respondent, when using income or wealth as SES measures, and by using biological data spanning longer durations, a more comprehensive analysis of pathways between SES and health status can add value to the disparities literature.

Other pathways through which SES may be related to health status and health outcomes should be examined. Proposed mediating variables may have interaction effects on health status or a complex feedback mechanism may arise in longitudinal relationships of variables. Future studies need to explore potential interaction effects on health status and health outcomes and examine feedback effects.

As an exploratory method, one of the strengths of RA is to generate hypotheses that can be studied further. The counterintuitive results in RA such as the differential 
effects of high BMI by race/ethnicity, the differential protective effects of being employed and volunteering in organizations are some of the hypotheses that future studies may address. 


\section{REFERENCES}

Adler, N. E., Boyce, T., Chesney, M. A., Cohen, S., Folkman, S., Kahn, R. L., \& Syme, S. L. (1994). Socioeconomic status and health. American Psychologist, 49(1), 1524.

Adler, N. E., \& Conner-Snibbe, A. (2003). The role of psychosocial processes in explaining the gradient between socioeconomic status and health. Current Directions in Psychological Science, 12(4), 119-123. https://doi.org/10.1111/1467-8721.01245

Adler, N. E., \& Newman, K. (2002). Socioeconomic Disparities In Health: Pathways And Policies. Health Affairs, 21(2), 60-76. https://doi.org/10.1377/hlthaff.21.2.60

Adler, N. E., \& Ostrove, J. M. (1999). Socioeconomic Status and Health: What We Know and What We Don't. Annals of the New York Academy of Sciences, 896(1), 3-15. https://doi.org/10.1111/j.1749-6632.1999.tb08101.x

Adler, N. E., \& Rehkopf, D. H. (2008). U.S. Disparities in Health: Descriptions, Causes, and Mechanisms. Annual Review of Public Health, 29(1), 235-252. https://doi.org/10.1146/annurev.publhealth.29.020907.090852

Agency for Healthcare Research and Quality. (2013, February). Minority Health: Recent Findings [Text]. Retrieved February 20, 2017, from /research/findings/factsheets/minority/minorfind/index.html

Ajrouch, K. J., Blandon, A. Y., \& Antonucci, T. C. (2005). Social networks among men and women: the effects of age and socioeconomic status. The Journals of Gerontology. Series B, Psychological Sciences and Social Sciences, 60(6), S311S317.

Angell, M. (1993). Privilege and Health - What Is the Connection? New England Journal of Medicine, 329(2), 126-127. https://doi.org/10.1056/NEJM199307083290210

Antonovsky, A. (1968). Social class and the major cardiovascular diseases. Journal of Chronic Diseases, 21(2), 65-106. https://doi.org/10.1016/0021-9681(68)90098-2

Ashby, W. R. (1964). Constraint Analysis of Many-Dimensional Relations. 
Asparouhov, T., \& Muthén, B. (2010). Weighted Least Squares Estimation with Missing Data. Retrieved from https://www.statmodel.com/download/GstrucMissingRevision.pdf

August, K. J., \& Sorkin, D. H. (2010). Racial and Ethnic Disparities in Indicators of Physical Health Status: Do They Still Exist Throughout Late Life? Journal of the American Geriatrics Society, 58(10), 2009-2015. https://doi.org/10.1111/j.15325415.2010.03033.x

Baron, R. M., \& Kenny, D. A. (1986). The moderator-mediator variable distinction in social psychological research: conceptual, strategic, and statistical considerations. Journal of Personality and Social Psychology, 51(6), 1173-1182.

Baum, A., Garofalo, J. P., \& Yali, A. (1999). Socioeconomic status and chronic stress: Does stress account for SES effects on health? Annals of the New York Academy of Sciences, 896(1), 131-144.

Ben-Shlomo, Y., \& Kuh, D. (2002). A life course approach to chronic disease epidemiology: conceptual models, empirical challenges and interdisciplinary perspectives. International Journal of Epidemiology, 31(2), 285-293. https://doi.org/10.1093/ije/31.2.285

Berkman, L. F., Glass, T., Brissette, I., \& Seeman, T. E. (2000). From social integration to health: Durkheim in the new millennium. Social Science \& Medicine, 51(6), 843-857.

Berkman, L. F., \& Syme, S. L. (1979). Social Networks, Host Resistance, and Mortality: A Nine-Year Follow-up Study of Alameda County Residents. American Journal of Epidemiology, 109(2), 186-204.

Bowen, M. E. (2010). Coronary Heart Disease from a Life-Course Approach: Findings from the Health and Retirement Study, 1998-2004. Journal of Aging and Health, 22(2), 219-241. https://doi.org/10.1177/0898264309355981

Bradley, R. H., \& Corwyn, R. F. (2002). Socioeconomic status and child development. Annual Review of Psychology, 53, 371-399. https://doi.org/10.1146/annurev.psych.53.100901.135233 
Braveman, P. (2006). Health Disparities and Health Equity: Concepts and Measurement. Annual Review of Public Health, 27(1), 167-194. https://doi.org/10.1146/annurev.publhealth.27.021405.102103

Bromberger, J. T., Harlow, S., Avis, N., Kravitz, H. M., \& Cordal, A. (2004). Racial/Ethnic Differences in the Prevalence of Depressive Symptoms Among Middle-Aged Women: The Study of Women's Health Across the Nation (SWAN). American Journal of Public Health, 94(8), 1378-1385.

Bromberger, J. T., \& Matthews, K. A. (1994). Employment status and depressive symptoms in middle-aged women: a longitudinal investigation. American Journal of Public Health, 84(2), 202-206.

Bunker, J. P., Gomby, D. S., \& Kehrer, B. H. (1989). Pathways to Health: The Role of Social Factors. Henry J. Kaiser Family Foundation.

Cain, L. D. (1964). Life Course and Social Structures. In R. E. L. Faris (Ed.), Chicago Handbook of Modern Sociology (pp. 272-309). Chicago, IL: Rand McNally \& Company.

Caspersen, C. J., Christenson, G. M., \& Pollard, R. A. (1986). Status of the 1990 physical fitness and exercise objectives-evidence from NHIS 1985. Public Health Reports, 101(6), 587.

Cassel, J. (1976). The contribution of the social environment to host resistance: the Fourth Wade Hampton Frost Lecture. American Journal of Epidemiology, 104(2), 107-123.

Cheong, J., MacKinnon, D. P., \& Khoo, S. T. (2003). Investigation of Mediational Processes Using Parallel Process Latent Growth Curve Modeling. Structural Equation Modeling : A Multidisciplinary Journal, 10(2), 238. https://doi.org/10.1207/S15328007SEM1002_5

Choudhury, S. (2002). Racial and Ethnic Differences in Wealth and Asset Choices (Bulletin). U. S. Social Security Administration. Retrieved from http://www.socialsecurity.gov/policy/docs/ssb/v64n4/v64n4p1.html 
Clausen, J. A. (1972). The life course of individuals. In M. Riley, W., M. Jonson, \& A. Foner (Eds.), Aging and Society (Vol. 3, pp. 457-514). New York: Russell Sage Found.

Cobb, S. (1976). Presidential Address-1976. Social support as a moderator of life stress. Psychosomatic Medicine, 38(5), 300-314.

Coburn, D., \& Pope, C. R. (1974). Socioeconomic Status and Preventive Health Behaviour. Journal of Health and Social Behavior, 15(2), 67-78. https://doi.org/10.2307/2137186

Cohen, S. (1988). Psychosocial models of the role of social support in the etiology of physical disease. Health Psychology: Official Journal of the Division of Health Psychology, American Psychological Association, 7(3), 269-297.

Cohen, S. (2004). Social relationships and health. American Psychologist, 59(8), 676.

Cohen, S., Doyle, W. J., Skoner, D. P., Rabin, B. S., \& Gwaltney, J. M. J. (1997). Social Ties and Susceptibility to the Common Cold. JAMA June 25, 1997, 277(24), 1940-1944.

Cohen, S., Underwood, L. G., \& Gottlieb, B. H. (2000). Social Support Measurement and Intervention: A Guide for Health and Social Scientists (1 edition). Oxford University Press, USA.

Cohen, S., \& Wills, T. A. (1985). Stress, social support, and the buffering hypothesis. Psychological Bulletin, 98(2), 310-357.

Cole, D. A., \& Maxwell, S. E. (2003). Testing Mediational Models with Longitudinal Data: Questions and Tips in the Use of Structural Equation Modeling. Journal of Abnormal Psychology, 112(4), 558-577. https://doi.org/10.1037/0021843X.112.4.558

Conger, R. D., \& Donnellan, M. B. (2007). An interactionist perspective on the socioeconomic context of human development. Annual Review of Psychology, 58, 175-199. https://doi.org/10.1146/annurev.psych.58.110405.085551

Cook, B. L., \& Manning, W. G. (2009). Measuring racial/ethnic disparities across the distribution of health care expenditures. Health Services Research, 44(5 Pt 1), 1603-1621. https://doi.org/10.1111/j.1475-6773.2009.01004.x 
Cwikel, J. M. G., Dielman, T. E., Kirscht, J. P., \& Israel, B. A. (1988). Mechanisms of Psychosocial Effects on Health: The Role of Social Integration, Coping Style and Health Behavior. Health Education \& Behavior, 15(2), 151-173. https://doi.org/10.1177/109019818801500202

Dannefer, D. (2003). Cumulative Advantage/Disadvantage and the Life Course: CrossFertilizing Age and Social Science Theory. The Journals of Gerontology: Series B, 58(6), S327-S337. https://doi.org/10.1093/geronb/58.6.S327

Diamond, A., \& Sekhon, J. S. (2013). Genetic Matching for Estimating Causal Effects: A General Multivariate Matching Method for Achieving Balance in Observational Studies, 95(3), 932-945.

Diez Roux, A. V., \& Mair, C. (2010). Neighborhoods and health. Annals of the New York Academy of Sciences, 1186(1), 125-145. https://doi.org/10.1111/j.17496632.2009.05333.x

Dohrenwend, B. P. (2000). The role of adversity and stress in psychopathology: some evidence and its implications for theory and research. Journal of Health and Social Behavior, 41(1), 1-19.

Duncan, T. E., Duncan, S., C., Strycker, L., A., Li, F., \& Alpert, A. (1999). An Introduction to Latent Variable Growth Curve Modeling: Concepts, Issues, and Applications. L. Erlbaum Associates.

Dunlop, D. D., Manheim, L. M., Song, J., \& Chang, R. W. (2002). Gender and ethnic/racial disparities in health care utilization among older adults. The Journals of Gerontology. Series B, Psychological Sciences and Social Sciences, 57(4), S221-233.

Eggers, P. W., \& Greenberg, L. G. (2000). Racial and ethnic differences in hospitalization rates among aged Medicare beneficiaries, 1998. Health Care Financing Review, 21(4), 91-105.

Elder, G. H. (1974). Children of the Great Depression: Social change in life experience. Chicago, IL: University of Chicago Press.

Elder, G. H., Jr. (1998). The Life Course as Developmental Theory. Child Development, 69(1), 1-12. https://doi.org/10.2307/1132065 
Enders, C. (2010). Applied Missing Data Analysis. New York: Guilford Press.

Ertel, K. A., Glymour, M. M., \& Berkman, L. F. (2008). Effects of social integration on preserving memory function in a nationally representative US elderly population. American Journal of Public Health, 98(7), 1215-1220.

Ertel, K. A., Glymour, M. M., \& Berkman, L. F. (2009). Social networks and health: A life course perspective integrating observational and experimental evidence. Journal of Social and Personal Relationships, 26(1), 73-92. https://doi.org/10.1177/0265407509105523

Evans, G. W. (2004). The environment of childhood poverty. The American Psychologist, 59(2), 77-92. https://doi.org/10.1037/0003-066X.59.2.77

Farmer, M. M., \& Ferraro, K. F. (2005). Are racial disparities in health conditional on socioeconomic status? Social Science \& Medicine, 60(1), 191-204. https://doi.org/10.1016/j.socscimed.2004.04.026

Federal Interagency Forum on Aging-Related Statistics. (2012). Older Americans 2012: Key Indicators of Well-Being. Washington, D.C.: Federal Interagency Forum on Aging-Related Statistics. Retrieved from http://www.agingstats.gov/Main_Site/Data/Data_2012.aspx

Ferraro, K. F., \& Shippee, T. P. (2009). Aging and Cumulative Inequality: How Does Inequality Get Under the Skin? The Gerontologist, 49(3), 333-343. https://doi.org/10.1093/geront/gnp034

Finney, S., J., \& DiStefano, C. (2006). Structural Equation Modeling: A Second Course. IAP.

Flegal, K. M., Carroll, M. D., Kit, B. K., \& Ogden, C. L. (2012). Prevalence of Obesity and Trends in the Distribution of Body Mass Index Among US Adults, 19992010. JAMA, 307(5), 491. https://doi.org/10.1001/jama.2012.39

Fonda, S., \& Herzog, R., A. (2004). Documentation of Physical Functioning Measured in the Health and Retirement Study and the Asset and Health Dynamics among the Oldest Old Study (HRS/AHEAD Documentation Report) (pp. 1-64). MI: Survey Research Center, University of Michigan. 
Gallo, W. T., Bradley, E. H., Dubin, J. A., Jones, R. N., Falba, T. A., Teng, H.-M., \& Kasl, S. V. (2006). The Persistence of Depressive Symptoms in Older Workers Who Experience Involuntary Job Loss: Results From the Health and Retirement Survey. The Journals of Gerontology Series B: Psychological Sciences and Social Sciences, 61(4), S221-S228.

Gaskin, D. J., \& Hoffman, C. (2000). Racial and ethnic differences in preventable hospitalizations across 10 states. Medical Care Research and Review: MCRR, 57 Suppl 1, 85-107.

Gelman, A., \& Hill, J. (2007). Data Analysis Using Regression and Multilevel/Hierarchical Models. Cambridge University Press.

Giddings, F. H. (1904). A theory of social causation. Publications of the American Economic Association, 5(2), 139-174.

Glass, T. A., Leon, C. F. M. D., Bassuk, S. S., \& Berkman, L. F. (2006). Social Engagement and Depressive Symptoms in Late Life Longitudinal Findings. Journal of Aging and Health, 18(4), 604-628. https://doi.org/10.1177/0898264306291017

Gottlieb, N. H., \& Green, L. W. (1984). Life Events, Social Network, Life-Style, and Health: An Analysis of the 1979 National Survey of Personal Health Practices and Consequences. Health Education \& Behavior, 11(1), 91-105. https://doi.org/10.1177/109019818401100105

Granovetter, M. (1973). The strength of weak ties. American Journal of Sociology, 78(6), 1360-1380.

Green, A. R., Carney, D. R., Pallin, D. J., Ngo, L. H., Raymond, K. L., Iezzoni, L. I., \& Banaji, M. R. (2007). Implicit bias among physicians and its prediction of thrombolysis decisions for black and white patients. Journal of General Internal Medicine, 22(9), 1231-1238. https://doi.org/10.1007/s11606-007-0258-5

Hansen, B. B., \& Klopfer, S. O. (2006). Optimal full matching and related designs via network flows, 15(3), 609-627. 
Heeringa, S. G., \& Connor, J. H. (1995). Technical Description of the Health and Retirement Survey Sample Design. University of Michigan. Retrieved from hrsonline.isr.umich.edu/sitedocs/userg/HRSSAMP.pdf

Ho, D., Imai, K., King, G., \& Stuart, E. (2007). Matching as nonparametric processing for reducing model dependence in parametric causal inference, (15), 199-236.

House, J. S., Kessler, R. C., \& Herzog, A. R. (1990). Age, socioeconomic status, and health. The Milbank Quarterly, 383-411.

House, J. S., Umberson, D., \& Landis, K. R. (1988). Structures and Processes of Social Support. Annual Review of Sociology, 14, 293-318.

House, J. S., \& Williams, D. R. (2000). Understanding and reducing socioeconomic and racial/ethnic disparities in health. In Promoting Health: Intervention Strategies from Social and Behavioral Research (pp. 81-124). Washington, D.C.: National Academies Press. Retrieved from http://www.nap.edu/openbook.php?record_id=9939\&page=81

Hu, L., \& Bentler, P. M. (1999). Cutoff criteria for fit indexes in covariance structure analysis: Conventional criteria versus new alternatives. Structural Equation Modeling: A Multidisciplinary Journal, 6(1), 1-55. https://doi.org/10.1080/10705519909540118

IBM Corp. (2012). IBM SPSS Statistics for Windows, Version 21.0. Armonk, NY: IBM Corp.

Institute for Social Research, University of Michigan. (2016). HRS Survey Design and Methodology | Health and Retirement Study. Retrieved May 21, 2017, from https://hrs.isr.umich.edu/documentation/survey-design

Institute of Medicine. (2006). Examining the Health Disparities Research Plan of the National Institutes of Health: Unfinished Business. (G. E. Thomson, F. Mitchell, \& M. B. Williams, Eds.). Washington (DC): National Academies Press (US). Retrieved from http://www.ncbi.nlm.nih.gov/books/NBK57043/ Institute of Medicine (US) Committee on Quality of Health Care in America. (2001). Crossing the Quality Chasm: A New Health System for the 21st Century. 
Washington (DC): National Academies Press (US). Retrieved from http://www.ncbi.nlm.nih.gov/books/NBK222274/

Jackson, J. S., Knight, K. M., \& Rafferty, J. A. (2010). Race and Unhealthy Behaviors: Chronic Stress, the HPA Axis, and Physical and Mental Health Disparities over the Life Course. American Journal of Public Health, 100(5), 933-939. https://doi.org/10.2105/AJPH.2008.143446

Jenkins, K., R., Ofstedal, M., B., \& Weir, D. (2008). Documentation of Health Behaviors and Risk Factors Measured in the Health and Retirement Study (HRS/AHEADA) (pp. 1-75). Ann Arbor, MI: Survey Research Center, University of Michigan.

Johnson, J. G., Cohen, P., Dohrenwend, B. P., Link, B. G., \& Brook, J. S. (1999). A longitudinal investigation of social causation and social selection processes involved in the association between socioeconomic status and psychiatric disorders. Journal of Abnormal Psychology, 108(3), 490-499.

Jones-Webb, R. J., Hsiao, C. Y., \& Hannan, P. (1995). Relationships between socioeconomic status and drinking problems among black and white men. Alcoholism, Clinical and Experimental Research, 19(3), 623-627.

Judd, C. M., \& Kenny, D. A. (1981). Estimating the Effects of Social Interventions. CUP Archive.

Kaniasty, K., \& Norris, F. H. (2008). Longitudinal linkages between perceived social support and posttraumatic stress symptoms: Sequential roles of social causation and social selection. Journal of Traumatic Stress, 21(3), 274-281. https://doi.org/10.1002/jts.20334

Kaplan, G. A. (1995). Where do shared pathways lead? Some reflections on a research agenda. Psychosomatic Medicine, 57(3), 208-212.

Kaplan, G. A., Haan, M., N., Syme, L. S., Minkler, M., \& Winkleby, M. (1987). Socioeconomic Status and Health, 3, 125-129.

Kaplan, G. A., Shema, S. J., \& Leite, M. C. A. (2008). Socioeconomic Determinants of Psychological Well-Being: The Role of Income, Income Change, and Income Sources Over 29 Years. Annals of Epidemiology, 18(7), 531-537. https://doi.org/10.1016/j.annepidem.2008.03.006 
Katz, S., Ford, A. B., Moskowitz, R. W., Jackson, B. A., \& Jaffe, M. W. (1963). Studies of Illness in the Aged: The Index of ADL: A Standardized Measure of Biological and Psychosocial Function. JAMA, 185(12), 914-919. https://doi.org/10.1001/jama.1963.03060120024016

Kaufman, J. S., \& Cooper, R. S. (2001). Commentary: Considerations for Use of Racial/Ethnic Classification in Etiologic Research. American Journal of Epidemiology, 154(4), 291-298. https://doi.org/10.1093/aje/154.4.291

Kawachi, I., Kennedy, B. P., Lochner, K., \& Prothrow-Stith, D. (1997). Social capital, income inequality, and mortality. American Journal of Public Health, 87(9), 1491-1498.

Klir, G. (1986). Reconstructability Analysis: An Offspring of Ashby's Constraint Theory, 3(4), 267-271.

Kramer, P., Westaway, S. K., Zwick, M., \& Shervais, S. (2012a). Reconstructability analysis of genetic loci associated with Alzheimer disease. In 2012 Joint 6th International Conference on Soft Computing and Intelligent Systems (SCIS) and 13th International Symposium on Advanced Intelligent Systems (ISIS) (pp. 21042110). https://doi.org/10.1109/SCIS-ISIS.2012.6505196

Kramer, P., Westaway, S. K., Zwick, M., \& Shervais, S. (2012b). Reconstructability analysis of genetic loci associated with Alzheimer disease. In The 6th International Conference on Soft Computing and Intelligent Systems, and The 13th International Symposium on Advanced Intelligence Systems (pp. 21042110). https://doi.org/10.1109/SCIS-ISIS.2012.6505196

Krause, N., \& Borawski-Clark, E. (1995). Social class differences in social support among older adults. The Gerontologist, 35(4), 498-508.

Krebs-Smith, S. M., Cook, A., Subar, A. F., Cleveland, L., \& Friday, J. (1995). US adults' fruit and vegetable intakes, 1989 to 1991: a revised baseline for the Healthy People 2000 objective. American Journal of Public Health, 85(12), $1623-1629$.

Krieger, N., Jr., C. Q., Peng, T., Horn-Ross, P., Stewart, S., Brown, S., .. Ward, F. (1999). Social Class, Race/Ethnicity, and Incidence of Breast, Cervix, Colon, 
Lung, and Prostate Cancer among Asian, Black, Hispanic, and White Residents of the San Francisco Bay Area, 1988-92 (United States). Cancer Causes \& Control, $10(6), 525-537$.

Krippendorff, K. (1986). Information Theory: Structural Models for Qualitative Data.

SAGE.

Kuh, D., \& Shlomo, Y. B. (2004). A Life Course Approach to Chronic Disease Epidemiology. Oxford University Press.

Laaksonen, M., Prättälä, R., Helasoja, V., Uutela, A., \& Lahelma, E. (2003). Income and health behaviours. Evidence from monitoring surveys among Finnish adults. Journal of Epidemiology and Community Health, 57(9), 711-717.

Langlie, J. K. (1977). Social Networks, Health Beliefs, and Preventive Health Behavior. Journal of Health and Social Behavior, 18(3), 244-260. https://doi.org/10.2307/2136352

LaVeist, T. A. (2005). Disentangling race and socioeconomic status: a key to understanding health inequalities. Journal of Urban Health: Bulletin of the New York Academy of Medicine, 82(2 Suppl 3), iii26-34. https://doi.org/10.1093/jurban/jti061

Lewis, T. T., Guo, H., Lunos, S., Mendes de Leon, C. F., Skarupski, K. A., Evans, D. A., \& Everson-Rose, S. A. (2011). Depressive symptoms and cardiovascular mortality in older black and white adults: evidence for a differential association by race. Circulation. Cardiovascular Quality and Outcomes, 4(3), 293-299. https://doi.org/10.1161/CIRCOUTCOMES.110.957548

Li, F., Harmer, P., Cardinal, B. J., Bosworth, M., \& Johnson-Shelton, D. (2009). Obesity and the Built Environment: Does the Density of Neighborhood Fast-Food Outlets Matter? American Journal of Health Promotion : AJHP, 23(3), 203-209.

Link, B. G., \& Phelan, J. (1995). Social conditions as fundamental causes of disease. Journal of Health and Social Behavior, Spec No, 80-94.

Linn, M. W., Sandifer, R., \& Stein, S. (1985). Effects of unemployment on mental and physical health. American Journal of Public Health, 75(5), 502-506. 
Lorant, V., Croux, C., Weich, S., Deliège, D., Mackenbach, J., \& Ansseau, M. (2007). Depression and socio-economic risk factors: 7-year longitudinal population study. The British Journal of Psychiatry, 190(4), 293-298. https://doi.org/10.1192/bjp.bp.105.020040

Lorant, V., Deliège, D., Eaton, W., Robert, A., Philippot, P., \& Ansseau, M. (2003). Socioeconomic Inequalities in Depression: A Meta-Analysis. American Journal of Epidemiology, 157(2), 98-112. https://doi.org/10.1093/aje/kwf182

Lynch, J. W., Kaplan, G. A., \& Salonen, J. T. (1997). Why do poor people behave poorly? Variation in adult health behaviours and psychosocial characteristics by stages of the socioeconomic lifecourse. Social Science \& Medicine, 44(6), 809819.

Lynch, J. W., Kaplan, G. A., \& Shema, S. J. (1997). Cumulative impact of sustained economic hardship on physical, cognitive, psychological, and social functioning. New England Journal of Medicine, 337(26), 1889-1895.

MacKinnon, D. (2008). Introduction to Statistical Mediation Analysis. Taylor \& Francis. MacKinnon, D. P. (2008). Introduction to Statistical Mediation Analysis. New York: Taylor \& Francis.

MacKinnon, D. P., Krull, J. L., \& Lockwood, C. M. (2000). Equivalence of the mediation, confounding and suppression effect. Prevention Science: The Official Journal of the Society for Prevention Research, 1(4), 173-181.

MacKinnon, D. P., Lockwood, C. M., Brown, C. H., Wang, W., \& Hoffman, J. M. (2007). The intermediate endpoint effect in logistic and probit regression. Clinical Trials (London, England), 4(5), 499-513. https://doi.org/10.1177/1740774507083434

MacKinnon, D. P., Lockwood, C. M., \& Williams, J. (2004). Confidence Limits for the Indirect Effect: Distribution of the Product and Resampling Methods. Multivariate Behavioral Research, 39(1), 99. https://doi.org/10.1207/s15327906mbr3901_4 
Maddox, G. L., \& Clark, D. O. (1992). Trajectories of Functional Impairment in Later Life. Journal of Health and Social Behavior, 33(2), 114-125. https://doi.org/10.2307/2137250

Marmot, M. G., Stansfeld, S., Patel, C., North, F., Head, J., White, I., ... Smith, G. D. (1991). Health inequalities among British civil servants: the Whitehall II study. The Lancet, 337(8754), 1387-1393. https://doi.org/10.1016/0140-6736 (91)93068-K

Mayr, F. B., Yende, S., Linde-Zwirble, W. T., Peck-Palmer, O. M., Barnato, A. E., Weissfeld, L. A., \& Angus, D. C. (2010). Infection rate and acute organ dysfunction risk as explanations for racial differences in severe sepsis. JAMA: The Journal of the American Medical Association, 303(24), 2495-2503. https://doi.org/10.1001/jama.2010.851

McEwen, B. S. (1998). Protective and damaging effects of stress mediators. New England Journal of Medicine, 338(3), 171-179.

McEwen, B. S., \& Stellar, E. (1993). Stress and the individual. Mechanisms leading to disease. Archives of Internal Medicine, 153(18), 2093-2101.

McGinnis, J. M., \& Foege, W. H. (1993). Actual causes of death in the United States. JAMA: The Journal of the American Medical Association, 270(18), 2207-2212.

McLeod, J. D., \& Kessler, R. C. (1990). Socioeconomic Status Differences in Vulnerability to Undesirable Life Events. Journal of Health and Social Behavior, 31(2), 162-172. https://doi.org/10.2307/2137170

Merton, R. K. (1968). The Matthew Effect in Science. Science, 159(3810), 56-63.

Merton, R. K. (1988). The Matthew effect in science, II: Cumulative advantage and the symbolism of intellectual property. Isis, 79(4), 606-623.

Miech, R. A., \& Shanahan, M. J. (2000). Socioeconomic Status and Depression over the Life Course. Journal of Health and Social Behavior, 41(2), 162-176. https://doi.org/10.2307/2676303

Mirowsky, J., \& Ross, C. E. (2003). Education, Social Status, and Health. Transaction Publishers. 
Mokdad, A. H., Marks, J. S., Stroup, D. F., \& Gerberding, J. L. (2004). Actual causes of death in the United States, 2000. JAMA: The Journal of the American Medical Association, 291(10), 1238-1245. https://doi.org/10.1001/jama.291.10.1238

Molina, K. M., Alegría, M., \& Mahalingam, R. (2013). A Multiple-Group Path Analysis of the Role of Everyday Discrimination on Self-Rated Physical Health among Latina/os in the USA. Annals of Behavioral Medicine, 45(1), 33-44. https://doi.org/10.1007/s12160-012-9421-2

Mulatu, M. S., \& Schooler, C. (2002). Causal connections between socio-economic status and health: reciprocal effects and mediating mechanisms. Journal of Health and Social Behavior, 43(1), 22-41.

Muthen, B. (1984). A General Structural Equation Model with Dichotomous, Ordered Categorical, and Continuous Latent Variable Indicators. Psychometrika, 49(1), 115-32.

Muthen, B., O., \& Curran, P., J. (1997). General Longitudinal Modeling of Individual Differences in Experimental Designs: A Latent Variable Framework for Analysis and Power Estimation, 2(4), 371-402.

Muthén, L. K., \& Muthén, B. O. (1998). Mplus User's Guide. Seventh Edition. Los Angeles, CA: Muthén \& Muthén.

Myers, H. F. (2008). Ethnicity- and socio-economic status-related stresses in context: an integrative review and conceptual model. Journal of Behavioral Medicine, 32(1), 9-19. https://doi.org/10.1007/s10865-008-9181-4

Myers, H. F., \& Huwang, W.-C. (2004). Cumulative psychosocial risks and resilience: A conceptual perspective on ethnic health disparities in late life. In Critical perspectives on racial and ethnic differences in health in late life. Washington, D.C.: National Academies Press.

National Research Council. (2003). Unequal Treatment: Confronting Racial and Ethnic Disparities in Health Care (full printed version). Washington, D.C.: The National Academies Press. Retrieved from http://www.nap.edu/catalog.php?record_id=10260 
National Research Council. (2004). Critical Perspectives on Racial and Ethnic Differences in Health in Late Life. Washington, D.C.: The National Academies Press. Retrieved from http://www.nap.edu/catalog.php?record_id=11086

Newsom, J. T. (2015). Longitudinal Structural Equation Modeling: A Comprehensive Introduction. New York: Routledge.

Novotny, T. E., Warner, K. E., Kendrick, J. S., \& Remington, P. L. (1988). Smoking by blacks and whites: socioeconomic and demographic differences. American Journal of Public Health, 78(9), 1187-1189.

Okun, M. A., August, K. J., Rook, K. S., \& Newsom, J. T. (2010). Does volunteering moderate the relation between functional limitations and mortality? Social Science \& Medicine, 71(9), 1662-1668. https://doi.org/10.1016/j.socscimed.2010.07.034

Oliver, M. L., \& Shapiro, T. M. (2006). Black Wealth, White Wealth: A New Perspective on Racial Inequality. Taylor \& Francis.

Ormel, J., Rijsdijk, F. V., Sullivan, M., van Sonderen, E., \& Kempen, G. I. J. M. (2002). Temporal and reciprocal relationship between IADL/ADL disability and depressive symptoms in late life. The Journals of Gerontology. Series B, Psychological Sciences and Social Sciences, 57(4), P338-347.

Ostrove, J. M., Feldman, P., \& Adler, N. E. (1999). Relations among Socioeconomic Status Indicators and Health for African-Americans and Whites. Journal of Health Psychology, 4(4), 451-463. https://doi.org/10.1177/135910539900400401

Ostrove, J. M., Feldman, P., \& Adler, N. E. (1999). Relations among Socioeconomic Status Indicators and Health for African-Americans and Whites. Journal of Health Psychology, 4(4), 451-463. https://doi.org/10.1177/135910539900400401

Pampel, F. C., Krueger, P. M., \& Denney, J. T. (2010). Socioeconomic Disparities in Health Behaviors. Annual Review of Sociology, 36(1), 349-370. https://doi.org/10.1146/annurev.soc.012809.102529

Pappas, G., Queen, S., Hadden, W., \& Fisher, G. (1993). The Increasing Disparity in Mortality between Socioeconomic Groups in the United States, 1960 and 1986. 
New England Journal of Medicine, 329(2), 103-109.

https://doi.org/10.1056/NEJM199307083290207

Pearlin, L. I., Menaghan, E. G., Lieberman, M. A., \& Mullan, J. T. (1981). The Stress

Process. Journal of Health and Social Behavior, 22(4), 337-356.

https://doi.org/10.2307/2136676

Pearlin, L. I., \& Schooler, C. (1978). The Structure of Coping. Journal of Health and Social Behavior, 19(1), 2-21. https://doi.org/10.2307/2136319

Phelan, J. C., \& Link, B. G. (2005). Controlling disease and creating disparities: a fundamental cause perspective. The Journals of Gerontology Series B: Psychological Sciences and Social Sciences, 60(Special Issue 2), S27-S33.

Pierce, J. P., Fiore, M. C., Novotny, T. E., Hatziandreu, E. J., \& Davis, R. M. (1989). Trends in cigarette smoking in the United States. Educational differences are increasing. JAMA: The Journal of the American Medical Association, 261(1), 5660 .

Pinquart, M., \& Sörensen, S. (2000). Influences of socioeconomic status, social network, and competence on subjective well-being in later life: a meta-analysis. Psychology and Aging, 15(2), 187-224.

Price, D. J. (1965). Networks of Scientific Papers. Science (New York, N.Y.), 149(3683), $510-515$.

Radloff, L. S. (1977). The CES-D Scale: A Self-Report Depression Scale for Research in the General Population. Applied Psychological Measurement, 1(3), 385-401. https://doi.org/10.1177/014662167700100306

Ross, C. E., \& Mirowsky, J. (1995). Does Employment Affect Health? Journal of Health and Social Behavior, 36(3), 230-243. https://doi.org/10.2307/2137340

Rubin, D. B. (1976a). Inference and missing data. Biometrika, 63(3), 581-592. https://doi.org/10.1093/biomet/63.3.581

Rubin, D. B. (1976b). Multivariate Matching Methods That are Equal Percent Bias Reducing, I: Some Examples. Biometrics, 32(1), 109-120. https://doi.org/10.2307/2529342 
Rubin, D. B. (1997). Estimating causal effects from large data sets using propensity scores. Annals of Internal Medicine, 127(8 Pt 2), 757-763.

Sapolsky, R. M. (1998). The Trouble With Testosterone: And Other Essays On The Biology Of The Human Predicament. Scribner.

Sapolsky, R. M. (2004). Social Status and Health in Humans and Other Animals. Annual Review of Anthropology, 33(1), 393-418. https://doi.org/10.1146/annurev.anthro.33.070203.144000

Schaefer, C., Coyne, J. C., \& Lazarus, R. S. (1981). The health-related functions of social support. Journal of Behavioral Medicine, 4(4), 381-406.

Schafer, J. L., \& Graham, J. W. (2002). Missing data: Our view of the state of the art. Psychological Methods, 7(2), 147-177. https://doi.org/10.1037/1082989X.7.2.147

Schneider EC, Zaslavsky AM, \& Epstein AM. (2002). Racial disparities in the quality of care for enrollees in Medicare managed care. JAMA, 287(10), 1288-1294. https://doi.org/10.1001/jama.287.10.1288

Seeman, T. E. (1996). Social ties and health: The benefits of social integration. Annals of Epidemiology, 6(5), 442-451. https://doi.org/10.1016/S1047-2797 (96)00095-6

Seeman, T. E., Bruce, M. L., \& McAvay, G. J. (1996). Social network characteristics and onset of ADL disability: MacArthur studies of successful aging. The Journals of Gerontology Series B: Psychological Sciences and Social Sciences, 51(4), S191S200.

Sekhon, J. S. (2011). Multivariate and Propensity Score Matching Software with Automated Balance Optimization: The Matching Package for R., 42(7), 1-52.

Selig, J. P., \& Preacher, K. J. (2009). Mediation models for longitudinal data in developmental research. Research in Human Development, 6(2-3), 144-164. https://doi.org/10.1080/15427600902911247

Shafer, A. B. (2006). Meta-analysis of the factor structures of four depression questionnaires: Beck, CES-D, Hamilton, and Zung. Journal of Clinical Psychology, 62(1), 123-146. https://doi.org/10.1002/jclp.20213 
Shervais, S., \& Zwick, M. (2003). Using reconstructability analysis to select input variables for artificial neural networks. In Proceedings of the International Joint Conference on Neural Networks, 2003 (Vol. 4, pp. 3022-3026 vol.4). https://doi.org/10.1109/IJCNN.2003.1224053

Singer, J. D., \& Willett, J. B. (2003). Applied Longitudinal Data Analysis: Modeling Change and Event Occurrence. Oxford University Press, USA.

Skarupski, K. A., Leon, C. F. M. de, Bienias, J. L., Barnes, L. L., Everson-Rose, S. A., Wilson, R. S., \& Evans, D. A. (2005). Black-White Differences in Depressive Symptoms among Older Adults Over Time. The Journals of Gerontology Series B: Psychological Sciences and Social Sciences, 60(3), P136-P142. https://doi.org/10.1093/geronb/60.3.P136

Sobel, M. E. (1982). Asymptotic Confidence Intervals for Indirect Effects in Structural Equation Models. Sociological Methodology, 13, 290-312. https://doi.org/10.2307/270723

Sobel, M. E. (1987). Direct and Indirect Effects in Linear Structural Equation Models. Sociological Methods \& Research, 16(1), 155-176. https://doi.org/10.1177/0049124187016001006

Stansfeld, S. A., Head, J., \& Marmot, M. G. (1997). Explaining social class differences in depression and well-being. Social Psychiatry and Psychiatric Epidemiology, 33(1), 1-9.

Stansfeld, S. A., Smith, G. D., \& Marmot, M. (1993). Association between physical and psychological morbidity in the Whitehall II Study. Journal of Psychosomatic Research, 37(3), 227-238.

StataCorp. (2013). Stata Statistical Software: Release 13. College Station, TX: StataCorp LP.

Sterling, P., \& Eyer, J. (1988). Allostasis: A New Paradigm to Explain Arousal Pathology. In S. Fisher \& J. Reason (Eds.), Handbook of life stress, cognition and health (Vol. xxxiii). Oxford, England: John Wiley \& Sons.

Sterling, P., \& Eyer, J. (1988). Allostasis: A new paradigm to explain arousal pathology. In Handbook of life stress, congition and health. John Wiley \& Sons, Ltd. 
Stringhini, S., Berkman, L., Dugravot, A., Ferrie, J. E., Marmot, M., Kivimaki, M., \& Singh-Manoux, A. (2012). Socioeconomic Status, Structural and Functional Measures of Social Support, and Mortality: The British Whitehall II Cohort Study, 1985-2009. American Journal of Epidemiology, 175(12), 1275-1283. https://doi.org/10.1093/aje/kwr461

Taylor, P., Kochhar, R., Fry, R., Velasco, G., \& Motel, S. (2011). Wealth Gaps Rise to Record Highs between Whites, Blacks, Hispanics. Pew Research Center. Retrieved from http://www.pewsocialtrends.org/2011/07/26/wealth-gaps-rise-torecord-highs-between-whites-blacks-hispanics/

Taylor, S. E., \& Seeman, T. E. (1999). Psychosocial resources and the SES-health relationship. Annals of the New York Academy of Sciences, 896(1), 210-225.

Thoits, P. A. (2011). Mechanisms Linking Social Ties and Support to Physical and Mental Health. Journal of Health and Social Behavior, 52(2), 145-161. https://doi.org/10.1177/0022146510395592

Turner, R. J., \& Marino, F. (1994). Social support and social structure: a descriptive epidemiology. Journal of Health and Social Behavior, 35(3), 193-212.

Uchino, B. N., Cacioppo, J. T., \& Kiecolt-Glaser, J. K. (1996). The relationship between social support and physiological processes: A review with emphasis on underlying mechanisms and implications for health. Psychological Bulletin, 119(3), 488-531. https://doi.org/10.1037/0033-2909.119.3.488

Urban Institute and Kaiser Commission on Medicaid and the Uninsured. (2012). Poverty Rate by Race/Ethnicity. Kaiser Family Foundation. Retrieved from http://kff.org/other/state-indicator/poverty-rate-by-raceethnicity/

U.S. Census Bureau. (2013). Decennial census of population, 1940 to 2010. Retrieved from https://www.census.gov/newsroom/cspan/1940census/CSPAN_1940slides.pdf

U.S. Department of Health and Human Services. (2000). Healthy people 2010: Understanding and Improving Health. Government Printing Office. van Kippersluis, H., \& Galama, T. (2014). Wealth and health behavior: Testing the concept of a health cost. European Economic Review, 72(C), 197-220. 
Van Kippersluis, H., \& Galama, T. J. (2013). Why the rich drink more but smoke less: the impact of wealth on health behaviors. Retrieved from https://papers.ssrn.com/sol3/papers.cfm?abstract_id=2252071

Vonneilich, N., Jöckel, K.-H., Erbel, R., Klein, J., Dragano, N., Siegrist, J., \& von dem Knesebeck, O. (2012). The mediating effect of social relationships on the association between socioeconomic status and subjective health - results from the Heinz Nixdorf Recall cohort study. BMC Public Health, 12(1), 285. https://doi.org/10.1186/1471-2458-12-285

Wallace, R. B., \& Herzog, A. R. (1995). Overview of the Health Measures in the Health and Retirement Study. The Journal of Human Resources, 30, S84-S107. https://doi.org/10.2307/146279

Whelan, C. T. (1993). The role of social support in mediating the psychological consequences of economic stress. Sociology of Health \& Illness, 15(1), 86-101. https://doi.org/10.1111/1467-9566.ep11343797

Whitehead, M. (1992). The concepts and principles of equity and health. International Journal of Health Services: Planning, Administration, Evaluation, 22(3), 429445.

Wilkinson, R. G. (1999). Health, hierarchy, and social anxiety. Annals of the New York Academy of Sciences, 896(1), 48-63.

Williams, D., \& Jackson, P. B. (2005). Social Sources of Racial Disparities in Health. Health Affairs, 24(2), 325-334. https://doi.org/10.1377/hlthaff.24.2.325

Williams, D. R. (1999). Race, socioeconomic status, and health. The added effects of racism and discrimination. Annals of the New York Academy of Sciences, 896, $173-188$.

Williams, D. R., \& Collins, C. (1995). US Socioeconomic and Racial Differences in Health: Patterns and Explanations. Annual Review of Sociology, 21(1), 349-386. https://doi.org/10.1146/annurev.so.21.080195.002025

Williams, D. R., Kontos, E. Z., Viswanath, K., Haas, J. S., Lathan, C. S., MacConaill, L. E., ... Ayanian, J. Z. (2012). Integrating Multiple Social Statuses in Health 
Disparities Research: The Case of Lung Cancer. Health Services Research, 47(3pt2), 1255-1277. https://doi.org/10.1111/j.1475-6773.2012.01404.X

Williams, D. R., Yu, Y., Jackson, J. S., \& Anderson, N. B. (1997). Racial Differences in Physical and Mental Health Socio-economic Status, Stress and Discrimination. Journal of Health Psychology, 2(3), 335-351. https://doi.org/10.1177/135910539700200305

Winkleby, M. A., Cubbin, C., Ahn, D. K., \& Kraemer, H. C. (1999). Pathways by which SES and ethnicity influence cardiovascular disease risk factors. Annals of the New York Academy of Sciences, 896(1), 191-209.

Wray, L. A., Alwin, D. F., McCammon, R. J., Manning, T., \& Best, L. E. (2006). Social status, risky health behaviors, and diabetes in middle-aged and older adults. The Journals of Gerontology. Series B, Psychological Sciences and Social Sciences, 61(6), S290-298.

Yang, Y. C., Li, T., \& Ji, Y. (2013). Impact of social integration on metabolic functions: evidence from a nationally representative longitudinal study of US older adults. BMC Public Health, 13, 1210. https://doi.org/10.1186/1471-2458-13-1210

Zimmer, Z., \& House, J. S. (2003). Education, income, and functional limitation transitions among American adults: contrasting onset and progression. International Journal of Epidemiology, 32(6), 1089-1097. https://doi.org/10.1093/ije/dyg254

Zwick, M. (2001). Wholes and Parts in General Systems Methodology. In G. P. Wagner (Ed.), The Character Concept in Evolutionary Biology (pp. 237-56). Academic Press.

Zwick, M. (2004). An overview of reconstructability analysis. Kybernetes, 33(5/6), 877905. https://doi.org/10.1108/03684920410533958

Zwick, M. (2011). Reconstructability Analysis of Epistasis. Annals of Human Genetics, 75(1), 157-171. https://doi.org/10.1111/j.1469-1809.2010.00628.x

Zwick, M., \& Shu, H. (1996). Set-Theoretic Reconstructability of Elementary Cellular Automata. In Advances in System Science and Application, Special Issue I (pp. 31-36). 


\section{APPENDICES}

\section{Appendix A: Survey Instrument}

Appendix A provides research questions and response options (in parentheses), where applicable, for the demographic variables, SES variables, social, psychological, behavioral as well as physical impairment and overnight hospitalization variables. All questions are from $2002-2010$ unless indicated otherwise. Further details on variable construction are provided in the methods section (Chapter 4).

\section{Independent Variables}

\section{Demographic factors}

Three demographic factors including race/ethnicity, gender and age were used in this study. From questions a and b below, a variable indicating Hispanic/Latino, White (Caucasian) and Black/African American race/ethnic groups were identified.

(a) Do you consider yourself Hispanic or Latino?

(b) Do you consider yourself primarily White or Caucasian, Black or African American, American Indian, or Asian?

(c) What is your gender?

(d) In what month, day, and year were you born?

2. Socioeconomic status (see pp. 34-35)

Note: Below, (b) was used in RA method and (c) was used in the SEM analyses.

a. What is the highest grade of school or year of college you completed? [IMPUTED] [1994 wave]

b. Now I'm going to ask you some questions about your current employment situation. Are you working now, temporarily laid off, unemployed and looking for work, disabled and unable to work, retired, a homemaker, or what?

c. Are you doing any work for pay at the present time?

d. Total household income [computed from several questions]

e. Total wealth less debts [computed from several questions] 


\section{Mediating Variables}

1. Social Integration (see pp. 34-35 for more information).

a. (/Do you have any relatives in or near the facility where you are living? /(Besides the people living here with you,) Do you have any relatives in your neighborhood?) (Yes/No)

b. (/Do you have any good friends in or near the facility?/Do you have any good friends living in your neighborhood?) (Yes/No)

c. How often do you get together with (/people in or near the facility/any of your neighbors) just to chat or for a social visit?

d. (How often do you get together with (/people in or near the facility/any of your neighbors) just to chat or for a social visit?)

e. Marital status had four categories: married, separated/divorced, widowed, and never married.

Note: The four category marital status variable used in RA was constructed from four separate questions in the HRS, and the two category variable married/not married=1/0 was used for the index in regression analysis.

f. Have you spent any time in the past 12 months doing volunteer work for religious, educational, health-related or other charitable organizations?

\section{Behavioral factors (see pp. 37-38 for more information)}

A BMI score was computed from question a, b and c. Alcohol consumption variable was computed using question $d$ and follow up questions i-ii. Smoking variable was computed from questions e and $\mathrm{f}$. Physical activity (exercise) was computed from question $\mathrm{g}$. Details are provided in the methods section.

a. About how tall are you? (in feet) [1992 wave]

b. About how tall are you? (in inches) [1992 wave]

c. About how much do you weigh?

d. Do you ever drink any alcoholic beverages such as beer, wine, or liquor? (Yes/NO)

If Yes: 
i. In the last three months, on average, how many days per week have you had any alcohol to drink? (For example, beer, wine, or any drink containing liquor.)

ii. In the last three months, on the days you drink, about how many drinks do you have?

e. Have you ever smoked cigarettes? (Yes/No) [1992 wave]

f. Do you smoke cigarettes now? (Yes/No)

g. We would like to know the type and amount of physical activity involved in your daily life. How often do you take part in sports or activities that are vigorous, such as running or jogging, swimming, cycling, aerobics or gym workout, tennis, or digging with a spade or shovel: more than once a week, once a week, one to three times a month, or hardly ever or never? [2004 to 2010 waves]

3. Psychological factors (see pp. 35-36 for more information)

In the question below, a, e and g make the negative affect, b, $\mathrm{c}$ and $\mathrm{h}$ make the somatic symptoms while $\mathrm{d}$ and $\mathrm{f}$ make the positive affect (reverse-coded) scales for the latent psychological variable and the three psychological variables for RA. See methods section for further information.

Question: Now think about the past week and the feelings you have experienced. Please tell me if each of the following was true for you much of the time during the past week. Much of the time during the past week:

a. You felt depressed. (Would you say yes or no?)

b. You felt that everything you did was an effort. (Would you say yes or no?)

c. Your sleep was restless. (Would you say yes or no?)

d. You were happy. (Would you say yes or no?)

e. You felt lonely. (Would you say yes or no?)

f. You enjoyed life. (Would you say yes or no?)

g. You felt sad. (Would you say yes or no?)

h. You could not get going. (Would you say yes or no?) 


\section{III.Health Outcome Variables}

Two outcome variables were used in in this study: overnight hospitalization and physical impairment. Dependence/independence in performing activities of daily living (ADL) was used as indicator of physical impairment in this study.

\section{Overnight hospitalization}

a. The next questions are about health care you have received. Since [PREV WAVE IW MONTH], [PREV WAVE IW YEAR]/Since [PREV WAVE IW YEAR]/In the last two years), have you been a patient in a hospital overnight? (Yes/No)

\section{Physical impairment status}

For operationalization of physical impairment outcome variable and calculation of the index, see p. 38-39 for more information.

a. Because of a health or memory problem do you have any difficulty with dressing, including putting on shoes and socks? (Yes/NO)

b. Because of a health or memory problem do you have any difficulty with walking across a room? (Yes/No)

c. (Because of a health or memory problem do you have any difficulty with) bathing or showering? (Yes/No)

d. (Because of a health or memory problem do you have any difficulty with) eating, such as cutting up your food? (Yes/No)

e. (Because of a health or memory problem do you have any difficulty with) getting in or out of bed? (Yes/No)

f. (Because of a health or memory problem do you have) any difficulty with using the toilet, including getting up and down? (Yes/No) 


\section{Appendix B: Additional SEM Results}

Table 47: Direct and indirect path coefficients for the social integration mediator-lagged path model Unstandardized coefficients (standard errors) and standardized coefficients.

\begin{tabular}{|c|c|c|c|c|}
\hline & Total & White & Black & Hispanic \\
\hline \multicolumn{5}{|c|}{ Direct effects of SES on physical impairment } \\
\hline Income $\rightarrow \mathrm{PI}_{\mathrm{t}=3}$ & $\begin{array}{l}-0.009(0.016) \\
-0.010\end{array}$ & $\begin{array}{l}0.001 \\
0.001\end{array}$ & $\begin{array}{l}-0.042(0.027) \\
-0.041\end{array}$ & $\begin{array}{l}-0.003(0.029) \\
-0.004\end{array}$ \\
\hline Wealth $\rightarrow \mathrm{PI}_{\mathrm{t}=3}$ & $\begin{array}{l}-0.015 * * *(0.004) \\
-0.054\end{array}$ & $\begin{array}{l}-0.014(0.007) \\
-0.046\end{array}$ & $\begin{array}{l}-0.018 *(0.008) \\
-0.07\end{array}$ & $\begin{array}{l}-0.012 *(0.006) \\
-0.048\end{array}$ \\
\hline $\mathrm{Job} \rightarrow \mathrm{PI}_{\mathrm{t}=3}$ & $\begin{array}{l}-0.129 * * *(0.032) \\
-0.052\end{array}$ & $\begin{array}{l}-0.107 *(0.034) \\
-0.046\end{array}$ & $\begin{array}{l}-0.212 *(0.08) \\
-0.066\end{array}$ & $\begin{array}{l}-0.440 * * *(0.109) \\
-0.14\end{array}$ \\
\hline Education $\rightarrow \mathrm{PI}_{\mathrm{t}=3}$ & $\begin{array}{l}-0.018 *(0.007) \\
-0.045\end{array}$ & $\begin{array}{l}-0.022 *(0.008) \\
-0.052\end{array}$ & $\begin{array}{l}0.011(0.014) \\
0.023\end{array}$ & $\begin{array}{l}-0.02(0.017) \\
-0.062\end{array}$ \\
\hline \multicolumn{5}{|c|}{ Direct effects of SES on social integration } \\
\hline Income $\rightarrow \mathrm{SI}_{\mathrm{t}=2}$ & $\begin{array}{l}0.058 * * *(0.014) \\
0.060\end{array}$ & 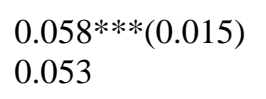 & $\begin{array}{l}0.043 *(0.022) \\
0.052\end{array}$ & $\begin{array}{l}0.034 *(0.016) \\
0.05\end{array}$ \\
\hline Wealth $\rightarrow \mathrm{SI}_{\mathrm{t}=2}$ & $\begin{array}{l}0.016 * * *(0.003) \\
0.055\end{array}$ & $\begin{array}{l}0.013 *(0.005) \\
0.041\end{array}$ & $\begin{array}{l}0.015 *(0.005) \\
0.074\end{array}$ & $\begin{array}{l}0.02 *(0.009) \\
0.092\end{array}$ \\
\hline $\mathrm{Job} \rightarrow \mathrm{SI}_{\mathrm{t}=2}$ & $\begin{array}{l}-0.050(0.032) \\
-0.020\end{array}$ & $\begin{array}{l}-0.055(0.037) \\
-0.022\end{array}$ & $\begin{array}{l}-0.054(0.067) \\
-0.021\end{array}$ & $\begin{array}{l}0.07(0.121) \\
0.027\end{array}$ \\
\hline Education $\rightarrow \mathrm{SI}_{\mathrm{t}=2}$ & $\begin{array}{l}0.010 *(0.004) \\
0.024\end{array}$ & $\begin{array}{l}0.003(0.005) \\
0.007\end{array}$ & $\begin{array}{l}0.01(0.015) \\
0.025\end{array}$ & $\begin{array}{l}0.015(0.012) \\
0.055\end{array}$ \\
\hline \multicolumn{5}{|c|}{ Effects of social integration on physical impairment } \\
\hline $\mathrm{SI}_{\mathrm{t}=2} \rightarrow \mathrm{PI}_{\mathrm{t}=3}$ & $\begin{array}{l}-0.048 * * *(0.012) \\
-0.050\end{array}$ & $\begin{array}{l}-0.057 * * *(0.014) \\
-0.062\end{array}$ & $\begin{array}{l}-0.029(0.032) \\
-0.024\end{array}$ & $\begin{array}{l}0.048(0.05) \\
0.04\end{array}$ \\
\hline \multicolumn{5}{|c|}{ Indirect effects of SES on physical impairment via social integration } \\
\hline Income $\rightarrow \mathrm{SI}_{\mathrm{t}=2} \rightarrow \mathrm{PI}_{\mathrm{t}=3}$ & $\begin{array}{l}-0.003 *(0.001) \\
-0.003\end{array}$ & $\begin{array}{l}-0.003 *(0.001) \\
-0.003\end{array}$ & $\begin{array}{l}-0.001(0.001) \\
-0.001\end{array}$ & $\begin{array}{l}0.002(0.002) \\
0.002\end{array}$ \\
\hline Wealth $\rightarrow \mathrm{SI}_{\mathrm{t}=2} \rightarrow \mathrm{PI}_{\mathrm{t}=3}$ & $\begin{array}{l}-0.001 *(0.000) \\
-0.003\end{array}$ & $\begin{array}{l}-0.001 *(0.000) \\
-0.003\end{array}$ & $\begin{array}{l}0.000(0.000) \\
-0.002\end{array}$ & $\begin{array}{l}0.001(0.001) \\
0.004\end{array}$ \\
\hline $\mathrm{Job} \rightarrow \mathrm{SI}_{\mathrm{t}=2} \rightarrow \mathrm{PI}_{\mathrm{t}=3}$ & $\begin{array}{l}0.002(0.002) \\
0.001\end{array}$ & $\begin{array}{l}0.003(0.002) \\
0.001\end{array}$ & $\begin{array}{l}0.002(0.003) \\
0.001\end{array}$ & $\begin{array}{l}0.003(0.007) \\
0.001\end{array}$ \\
\hline Education $\rightarrow \mathrm{SI}_{\mathrm{t}=2} \rightarrow \mathrm{PI}_{\mathrm{t}=3}$ & $\begin{array}{l}-0.00 *(0.000) \\
-0.001\end{array}$ & $\begin{array}{l}0.000(0.000) \\
0.000\end{array}$ & $\begin{array}{l}0.00(0.001) \\
-0.001\end{array}$ & $\begin{array}{l}0.001(0.001) \\
0.002\end{array}$ \\
\hline
\end{tabular}

Note: $*<.05, * * *<.001$. SI=social integration; $\mathrm{PI}=$ physical impairment $\&_{\mathrm{t}}=$ time indicator. 
Table 48: Direct and indirect path coefficients for the social integration mediator-latent growth curve model

Unstandardized coefficients (standard errors) and standardized coefficients.

\begin{tabular}{|c|c|c|c|c|}
\hline & Total & White & Black & Hispanic \\
\hline \multicolumn{5}{|c|}{ Direct effects of SES on the slope factor for physical impairment } \\
\hline Income $\rightarrow \mathrm{PI}_{\text {slope }}$ & $\begin{array}{l}-0.006(0.004) \\
-0.042\end{array}$ & $\begin{array}{l}-0.003(0.005) \\
-0.017\end{array}$ & $\begin{array}{l}-0.006(0.006) \\
-0.038\end{array}$ & $\begin{array}{l}-0.013(0.01) \\
-0.105\end{array}$ \\
\hline Wealth $\rightarrow \mathrm{PI}_{\text {slope }}$ & $\begin{array}{l}-0.003 *(0.001) \\
-0.074\end{array}$ & $\begin{array}{l}-0.005 *(0.002) \\
-0.109\end{array}$ & $\begin{array}{l}0.003(0.003) \\
-0.067\end{array}$ & $\begin{array}{l}0.005(0.003) \\
0.121\end{array}$ \\
\hline Job $\rightarrow \mathrm{PI}_{\text {slope }}$ & $\begin{array}{l}-0.012(0.007) \\
-0.031\end{array}$ & $\begin{array}{l}-0.010(0.008) \\
-0.026\end{array}$ & $\begin{array}{l}-0.049(0.027) \\
-0.092\end{array}$ & $\begin{array}{l}-0.050 *(0.023) \\
-0.105\end{array}$ \\
\hline Education $\rightarrow \mathrm{PI}_{\text {slope }}$ & $\begin{array}{l}-0.003 *(0.001) \\
-0.056\end{array}$ & $\begin{array}{l}-0.003(0.002) \\
-0.043\end{array}$ & $\begin{array}{l}0.002(0.006) \\
0.019\end{array}$ & $\begin{array}{l}-0.010 *(0.004) \\
-0.203\end{array}$ \\
\hline
\end{tabular}

Effects of slope factor for social integration on the slope factor of physical impairment

\begin{tabular}{|c|c|c|c|c|}
\hline$S I_{\text {slope }} \rightarrow \mathrm{PI}_{\text {slope }}$ & $\begin{array}{l}-0.168 * * *(0.035) \\
-0.173\end{array}$ & $\begin{array}{l}0.163 * * *(0.033) \\
-0.182\end{array}$ & $\begin{array}{l}-0.302(0.16) \\
-0.203\end{array}$ & $\begin{array}{l}-0.003(0.182) \\
-0.003\end{array}$ \\
\hline
\end{tabular}

Direct effects of SES on the slope factor for social integration

$\begin{array}{lllll}\text { Income } \rightarrow S I_{\text {slope }} & 0.00(0.004) & 0.00(0.005) & 0.003(0.006) & -0.013(0.009) \\ & 0.024 & -0.002 & 0.024 & -0.125 \\ & & & & \\ \text { Wealth } \rightarrow S I_{\text {slope }} & 0.001(0.001) & 0.001(0.001) & -0.001(0.003) & 0.001(0.002) \\ & 0.000 & 0.027 & -0.037 & 0.018 \\ & & & & \\ \mathrm{Job} \rightarrow S I_{\text {slope }} & 0.042^{*}(0.009) & 0.046^{* * *}(0.01) & -0.008(0.022) & 0.096^{* * *}(0.03) \\ & 0.106 & 0.114 & -0.023 & 0.24 \\ \text { Education } \rightarrow S I_{\text {slope }} & 0.005^{*}(0.001) & 0.004^{*}(0.002) & 0.004(0.002) & 0.002(0.005) \\ & 0.071 & 0.056 & 0.075 & 0.052\end{array}$

Indirect effects of SES on the slope factor for physical impairment via slope of social integration

$\begin{array}{cllll}\text { Income } \rightarrow S I_{\text {slope }} \rightarrow \mathrm{PI}_{\text {slope }} & 0(0.001) & 0.00(0.001) & -0.001(0.002) & 0.00(0.002) \\ & 0.000 & 0.000 & -0.005 & 0.000 \\ \text { Wealth } \rightarrow S I_{\text {slope }} \rightarrow \mathrm{PI}_{\text {slope }} & -0.00(0.000) & 0.00(0.000) & 0.00(0.001) & 0.00(0.000) \\ & -0.004 & -0.005 & 0.007 & 0.000 \\ \mathrm{Job} \rightarrow S I_{\text {slope }} \rightarrow P I_{\text {slope }} & -0.018^{* * *}(0.002) & -0.007 *(0.002) & 0.002(0.007) & 0.00(0.017) \\ & -0.018 & -0.21 & 0.005 & -0.001 \\ & -0.012 *(0.000) & -0.001 *(0.000) & -0.001(0.001) & 0.00(0.000) \\ \text { Education } \rightarrow S I_{\text {slope }} \rightarrow \mathrm{PI}_{\text {slope }} & -0.012 & -0.01 & -0.015 & 0.000\end{array}$

Note: $\mathrm{p}<0.05^{*}, 0.001^{* * *}, \mathrm{SI}=$ social integration and $\mathrm{PI}=$ physical impairment slope factors. 
Table 49: Direct and indirect path coefficients for the depressive symptoms mediator-lagged path model Unstandardized coefficients (standard errors) and standardized coefficients.

\begin{tabular}{|c|c|c|c|c|}
\hline & Total & White & Black & Hispanic \\
\hline \multicolumn{5}{|c|}{ Direct effects of SES on physical impairment } \\
\hline Income $\rightarrow \mathrm{PI}_{\mathrm{t}=3}$ & $\begin{array}{l}-0.005(0.016) \\
-0.006\end{array}$ & $\begin{array}{l}0.015(0.019) \\
0.015\end{array}$ & $\begin{array}{l}-0.034(0.027) \\
-0.033\end{array}$ & $\begin{array}{l}-0.043(0.03) \\
-0.053\end{array}$ \\
\hline Wealth $\rightarrow \mathrm{PI}_{\mathrm{t}=3}$ & $\begin{array}{l}-0.012 * * *(0.017) \\
-0.045\end{array}$ & $\begin{array}{l}-0.011(0.007) \\
-0.038\end{array}$ & $\begin{array}{l}-0.016(0.009) \\
-0.065\end{array}$ & $\begin{array}{l}-0.003(0.007) \\
-0.012\end{array}$ \\
\hline $\mathrm{Job} \rightarrow \mathrm{PI}_{\mathrm{t}=3}$ & $\begin{array}{l}-0.099 * * *(0.012) \\
-0.04\end{array}$ & $\begin{array}{l}-0.079 *(0.033) \\
-0.034\end{array}$ & $\begin{array}{l}-0.182 *(0.08) \\
-0.056\end{array}$ & $\begin{array}{l}-0.412 * * *(0.101) \\
-0.131\end{array}$ \\
\hline Education $\rightarrow \mathrm{PI}_{\mathrm{t}=3}$ & $\begin{array}{l}-0.012 *(0.019) \\
-0.031\end{array}$ & $\begin{array}{l}-0.016^{*}(0.008) \\
-0.038\end{array}$ & $\begin{array}{l}0.023(0.014) \\
0.046\end{array}$ & $\begin{array}{l}-0.017(0.016) \\
-0.051\end{array}$ \\
\hline \multicolumn{5}{|c|}{ Direct effects of SES on depressive symptoms } \\
\hline Income $\rightarrow \mathrm{DS}_{\mathrm{t}=2}$ & $\begin{array}{l}-0.005(0.02) \\
-0.032\end{array}$ & $\begin{array}{l}-0.007 *(0.003) \\
-0.041\end{array}$ & $\begin{array}{l}0.006(0.003) \\
0.04\end{array}$ & $\begin{array}{l}-0.006(0.008) \\
-0.043\end{array}$ \\
\hline Wealth $\rightarrow \mathrm{DS}_{\mathrm{t}=2}$ & $\begin{array}{l}-0.003 * * *(0.017) \\
-0.069\end{array}$ & $\begin{array}{l}-0.003 * * *(0.001) \\
-0.068\end{array}$ & $\begin{array}{l}-0.002(0.002) \\
-0.051\end{array}$ & $\begin{array}{l}-0.004 *(0.002) \\
-0.1\end{array}$ \\
\hline $\mathrm{Job} \rightarrow \mathrm{DS}_{\mathrm{t}=2}$ & $\begin{array}{l}-0.014 *(0.011) \\
-0.034\end{array}$ & $\begin{array}{l}-0.013 *(0.005) \\
-0.033\end{array}$ & $\begin{array}{l}-0.028 *(0.012) \\
-0.063\end{array}$ & $\begin{array}{l}-0.006(0.028) \\
-0.011\end{array}$ \\
\hline Education $\rightarrow \mathrm{DS}_{\mathrm{t}=2}$ & $\begin{array}{l}-0.005^{* * *}(0.015) \\
-0.07\end{array}$ & $\begin{array}{l}-0.004 *(0.001) \\
-0.05\end{array}$ & $\begin{array}{l}-0.006 *(0.002) \\
-0.093\end{array}$ & $\begin{array}{l}-0.006 *(0.003) \\
-0.101\end{array}$ \\
\hline \multicolumn{5}{|c|}{ Effects of depressive symptoms on physical impairment } \\
\hline $\mathrm{DS}_{\mathrm{t}=2} \rightarrow \mathrm{PI}_{\mathrm{t}=3}$ & $\begin{array}{l}0.675^{* * *}(0.121) \\
0.114\end{array}$ & $\begin{array}{l}0.596 * * *(0.131) \\
0.103\end{array}$ & $\begin{array}{l}0.908(0.55) \\
0.125\end{array}$ & $\begin{array}{l}1.23 * * *(0.295) \\
0.214\end{array}$ \\
\hline \multicolumn{5}{|c|}{ Indirect effects of SES on physical impairment via depressive symptoms } \\
\hline Income $\rightarrow \mathrm{DS}_{\mathrm{t}=2} \rightarrow \mathrm{PI}_{\mathrm{t}=3}$ & $\begin{array}{l}-0.003(0.002) \\
-0.004\end{array}$ & $\begin{array}{l}-0.004 *(0.002) \\
-0.004\end{array}$ & $\begin{array}{l}0.005(0.007) \\
0.005\end{array}$ & $\begin{array}{l}-0.007(0.011) \\
-0.009\end{array}$ \\
\hline Wealth $\rightarrow \mathrm{DS}_{\mathrm{t}=2} \rightarrow \mathrm{PI}_{\mathrm{t}=3}$ & $\begin{array}{l}-0.002 * *(0.001) \\
-0.008\end{array}$ & $\begin{array}{l}-0.002 *(0.001) \\
-0.007\end{array}$ & $\begin{array}{l}-0.002(0.002) \\
-0.006\end{array}$ & $\begin{array}{l}-0.005 *(0.002) \\
-0.021\end{array}$ \\
\hline $\mathrm{Job} \rightarrow \mathrm{DS}_{\mathrm{t}=2} \rightarrow \mathrm{PI}_{\mathrm{t}=3}$ & $\begin{array}{l}-0.01 *(0.003) \\
-0.008\end{array}$ & $\begin{array}{l}-0.008 *(0.001) \\
-0.003\end{array}$ & $\begin{array}{l}-0.025(0.004) \\
-0.008\end{array}$ & $\begin{array}{l}-0.007(0.003) \\
-0.002\end{array}$ \\
\hline Education $\rightarrow \mathrm{DS}_{\mathrm{t}=2} \rightarrow \mathrm{PI}_{\mathrm{t}=3}$ & $\begin{array}{l}-0.003 * * *(0.001) \\
-0.004\end{array}$ & $\begin{array}{l}-0.002 *(0.003) \\
-0.005\end{array}$ & $\begin{array}{l}-0.006(0.017) \\
-0.012\end{array}$ & $\begin{array}{l}-0.007 *(0.034) \\
-0.022\end{array}$ \\
\hline
\end{tabular}

Note: $\mathrm{p} *<0.05, * * *<0.001 . \mathrm{DS}=$ depressive symptoms; $\mathrm{PI}=$ physical impairment $\&_{\mathrm{t}}=$ time. 
Table 50: Direct and indirect path coefficients for the depressive symptoms mediator-latent growth curve model

Unstandardized coefficients (standard errors) and standardized coefficients

\begin{tabular}{|c|c|c|c|c|}
\hline & Total & White & Black & Hispanic \\
\hline \multicolumn{5}{|c|}{ Direct effects of SES on the slope factor for physical impairment } \\
\hline Income $\rightarrow \mathrm{PI}_{\text {slope }}$ & $\begin{array}{l}-0.011 *(0.005) \\
-0.071\end{array}$ & $\begin{array}{l}-0.004(0.006) \\
-0.028\end{array}$ & $\begin{array}{l}-0.037 *(0.015) \\
-0.224\end{array}$ & $\begin{array}{l}-0.017(0.021) \\
-0.135\end{array}$ \\
\hline Wealth $\rightarrow \mathrm{PI}_{\text {slope }}$ & $\begin{array}{l}-0.005 *(0.002) \\
-0.111\end{array}$ & $\begin{array}{l}-0.007 *(0.002) \\
-0.138\end{array}$ & $\begin{array}{l}-0.002(0.004) \\
-0.058\end{array}$ & $\begin{array}{l}-0.001(0.006) \\
-0.033\end{array}$ \\
\hline $\mathrm{Job} \rightarrow \mathrm{PI}_{\text {slope }}$ & $\begin{array}{l}-0.028 * *(0.009) \\
-0.071\end{array}$ & $\begin{array}{l}-0.031 * * *(0.009) \\
-0.064\end{array}$ & $\begin{array}{l}-0.029(0.036) \\
-0.057\end{array}$ & $\begin{array}{l}-0.123(0.067) \\
-0.281\end{array}$ \\
\hline Education $\rightarrow \mathrm{PI}_{\text {slope }}$ & $\begin{array}{l}-0.004 *(0.002) \\
-0.063\end{array}$ & $\begin{array}{l}-0.002(0.002) \\
-0.063\end{array}$ & $\begin{array}{l}0.006(0.005) \\
0.079\end{array}$ & $\begin{array}{l}-0.006(0.007) \\
-0.119\end{array}$ \\
\hline \multicolumn{5}{|c|}{ Effects of slope factor for depressive symptoms on the slope factor for physical impairment } \\
\hline$D S_{\text {slope }} \rightarrow \mathrm{PI}_{\text {slope }}$ & $\begin{array}{l}3.49 * * *(0.923) \\
0.415\end{array}$ & $\begin{array}{l}2.36 *(0.969) \\
0.300\end{array}$ & $\begin{array}{l}8.46(4.44) \\
0.838\end{array}$ & $\begin{array}{l}5.63(4.99) \\
0.763\end{array}$ \\
\hline \multicolumn{5}{|c|}{ Direct effects of SES on the slope factor for depressive symptoms } \\
\hline Income $\rightarrow D S_{\text {slope }}$ & $\begin{array}{l}0.001(0.001) \\
0.073\end{array}$ & $\begin{array}{l}0.001(0.001) \\
0.042\end{array}$ & $\begin{array}{l}0.004 *(0.002) \\
0.218\end{array}$ & $\begin{array}{l}0.001(0.003) \\
0.044\end{array}$ \\
\hline Wealth $\rightarrow D S_{\text {slope }}$ & $\begin{array}{l}0.000(0.00) \\
0.085\end{array}$ & $\begin{array}{l}0.001(0.001) \\
0.090\end{array}$ & $\begin{array}{l}-0.00(0.00) \\
-0.002\end{array}$ & $\begin{array}{l}0.001(0.001) \\
0.196\end{array}$ \\
\hline $\mathrm{Job} \rightarrow D S_{\text {slope }}$ & $\begin{array}{l}0.002(0.002) \\
0.053\end{array}$ & $\begin{array}{l}0.003(0.002) \\
0.056\end{array}$ & $\begin{array}{l}-0.002(0.003) \\
-0.036\end{array}$ & $\begin{array}{l}0.012(0.008) \\
0.22\end{array}$ \\
\hline Education $\rightarrow D S_{\text {slope }}$ & $\begin{array}{l}0.000(0.00) \\
-0.017\end{array}$ & $\begin{array}{l}0.000(0.00) \\
0.030\end{array}$ & $\begin{array}{l}-0.001(0.001) \\
-0.088\end{array}$ & $\begin{array}{l}-0.001(0.001) \\
-0.109\end{array}$ \\
\hline \multicolumn{5}{|c|}{ Indirect effects of SES on the slope factor for physical impairment via slope of depressive symptoms } \\
\hline Income $\rightarrow D S_{\text {slope }} \rightarrow \mathrm{PI}_{\text {slope }}$ & $\begin{array}{l}0.005(0.004) \\
0.03\end{array}$ & $\begin{array}{l}0.002(0.003) \\
0.013\end{array}$ & $\begin{array}{l}0.030(0.016) \\
0.177\end{array}$ & $\begin{array}{l}0.005(0.017) \\
0.039\end{array}$ \\
\hline Wealth $\rightarrow D S_{\text {slope }} \rightarrow \mathrm{PI}_{\text {slope }}$ & $\begin{array}{l}0.002(0.001) \\
0.035\end{array}$ & $\begin{array}{l}0.001(0.001) \\
0.028\end{array}$ & $\begin{array}{l}-0.00(0.004) \\
-0.007\end{array}$ & $\begin{array}{l}0.005(0.005) \\
0.132\end{array}$ \\
\hline $\mathrm{Job} \rightarrow D S_{\text {slope }} \rightarrow \mathrm{PI}_{\text {slope }}$ & $\begin{array}{l}0.009(0.006) \\
0.022\end{array}$ & $\begin{array}{l}0.006(0.005) \\
0.017\end{array}$ & $\begin{array}{l}-0.018(0.028) \\
-0.034\end{array}$ & $\begin{array}{l}0.069(0.081) \\
0.144\end{array}$ \\
\hline Education $\rightarrow D S_{\text {slope }} \rightarrow \mathrm{PI}_{\text {slope }}$ & $\begin{array}{l}0.00(0.001) \\
-0.007\end{array}$ & $\begin{array}{l}0.001(0.001) \\
0.009\end{array}$ & $\begin{array}{l}-0.006(0.005) \\
-0.071\end{array}$ & $\begin{array}{l}-0.004(0.006) \\
-0.073\end{array}$ \\
\hline
\end{tabular}

Note: $\mathrm{p}<0.05^{*}, 0.001^{* * *}$. DS=depressive symptoms and $\mathrm{PI}=$ physical impairment slope factors. 
Table 51: Direct and indirect path coefficients for BMI and alcoholic beverage use mediators-lagged path model

Unstandardized coefficients (standard errors) and standardized coefficients.

\begin{tabular}{|c|c|c|c|c|}
\hline & Total & White & Black & Hispanic \\
\hline \multicolumn{5}{|c|}{ Direct effects of SES on physical impairment } \\
\hline Income $\rightarrow \mathrm{PI}_{\mathrm{t}=3}$ & $\begin{array}{l}-0.016(0.016) \\
-0.018\end{array}$ & $\begin{array}{l}-0.009(0.02) \\
-0.009\end{array}$ & $\begin{array}{l}-0.022(0.028) \\
-0.021\end{array}$ & $\begin{array}{l}-0.025(0.027) \\
-0.031\end{array}$ \\
\hline Wealth $\rightarrow \mathrm{PI}_{\mathrm{t}=3}$ & $\begin{array}{l}-0.016 * * *(0.004) \\
-0.057\end{array}$ & $\begin{array}{l}-0.014(0.007) \\
-0.049\end{array}$ & $\begin{array}{l}-0.018 *(0.008) \\
-0.072\end{array}$ & $\begin{array}{l}-0.011(0.006) \\
-0.043\end{array}$ \\
\hline $\mathrm{Job} \rightarrow \mathrm{PI}_{\mathrm{t}=3}$ & $\begin{array}{l}-0.121 * * *(0.031) \\
-0.049\end{array}$ & $\begin{array}{l}-0.095 *(0.032) \\
-0.041\end{array}$ & $\begin{array}{l}-0.212 *(0.083) \\
-0.066\end{array}$ & $\begin{array}{l}-0.427 * * *(0.105) \\
-0.136\end{array}$ \\
\hline Education $\rightarrow \mathrm{PI}_{\mathrm{t}=3}$ & $\begin{array}{l}-0.018 *(0.007) \\
-0.045\end{array}$ & $\begin{array}{l}-0.022 *(0.008) \\
-0.051\end{array}$ & $\begin{array}{l}0.015(0.013) \\
0.03\end{array}$ & $\begin{array}{l}-0.022(0.017) \\
-0.066\end{array}$ \\
\hline \multicolumn{5}{|c|}{ Direct effects of SES on behavioral variables (BMI \& ALC) } \\
\hline Income $\rightarrow \mathrm{BMI}_{\mathrm{t}=2}$ & $\begin{array}{l}-0.033(0.049) \\
-0.008\end{array}$ & $\begin{array}{l}-0.014(0.062) \\
-0.003\end{array}$ & $\begin{array}{l}-0.135(0.111) \\
-0.032\end{array}$ & $\begin{array}{l}0.006(0.062) \\
0.002\end{array}$ \\
\hline Wealth $\rightarrow$ BMI $_{\mathrm{t}=2}$ & $\begin{array}{l}-0.028 *(0.011) \\
-0.022\end{array}$ & $\begin{array}{l}-0.041 *(0.015) \\
-0.029\end{array}$ & $\begin{array}{l}0.012(0.015) \\
0.011\end{array}$ & $\begin{array}{l}-0.038(0.025) \\
-0.042\end{array}$ \\
\hline $\mathrm{Job} \rightarrow \mathrm{BMI}_{\mathrm{t}=2}$ & $\begin{array}{l}0.057(0.071) \\
0.005\end{array}$ & $\begin{array}{l}0.045(0.082) \\
0.004\end{array}$ & $\begin{array}{l}0.254(0.178) \\
0.019\end{array}$ & $\begin{array}{l}-0.195(0.301) \\
-0.018\end{array}$ \\
\hline Education $\rightarrow \mathrm{BMI}_{\mathrm{t}=2}$ & $\begin{array}{l}0.014(0.016) \\
0.008\end{array}$ & $\begin{array}{l}-0.002(0.017) \\
-0.001\end{array}$ & $\begin{array}{l}0.072(0.05) \\
0.036\end{array}$ & $\begin{array}{l}0.034(0.048) \\
0.03\end{array}$ \\
\hline Income $\rightarrow \mathrm{ALC}_{\mathrm{t}=2}$ & $\begin{array}{l}0.014(0.008) \\
0.02\end{array}$ & $\begin{array}{l}0.008(0.008) \\
0.01\end{array}$ & $\begin{array}{l}0.005(0.007) \\
0.012\end{array}$ & $\begin{array}{l}0.02 *(0.007) \\
0.064\end{array}$ \\
\hline Wealth $\rightarrow$ ALC $_{t=2}$ & $\begin{array}{l}0.003 * ?(0.001) \\
0.013\end{array}$ & $\begin{array}{l}0.004(0.002) \\
0.015\end{array}$ & $\begin{array}{l}0.001(0.002) \\
0.005\end{array}$ & $\begin{array}{l}-0.004(0.003) \\
-0.046\end{array}$ \\
\hline $\mathrm{Job} \rightarrow \mathrm{ALC}_{\mathrm{t}=2}$ & $\begin{array}{l}-0.039 *(0.019) \\
-0.021\end{array}$ & $\begin{array}{l}-0.037(0.02) \\
-0.019\end{array}$ & $\begin{array}{l}-0.049(0.049) \\
-0.034\end{array}$ & $\begin{array}{l}0.032(0.044) \\
0.027\end{array}$ \\
\hline Education $\rightarrow \mathrm{ALC}_{\mathrm{t}=2}$ & $\begin{array}{l}0.01 *(0.003) \\
0.033\end{array}$ & $\begin{array}{l}0.008(0.004) \\
0.022\end{array}$ & $\begin{array}{l}0.009(0.006) \\
0.041\end{array}$ & $\begin{array}{l}0.004(0.004) \\
0.029\end{array}$ \\
\hline \multicolumn{5}{|c|}{ Effects of behavioral variables (BMI \& ALC) on physical impairment } \\
\hline $\mathrm{BMI}_{\mathrm{t}=2} \rightarrow \mathrm{PI}_{\mathrm{t}=3}$ & $\begin{array}{l}0.011 *(0.004) \\
0.051\end{array}$ & $\begin{array}{l}0.011 *(0.004) \\
0.052\end{array}$ & $\begin{array}{l}0.001(0.006) \\
0.003\end{array}$ & $\begin{array}{l}0.026 *(0.011) \\
0.091\end{array}$ \\
\hline $\mathrm{ALC}_{\mathrm{t}=2} \rightarrow \mathrm{PI}_{\mathrm{t}=3}$ & $\begin{array}{l}0.03(0.025) \\
0.023\end{array}$ & $\begin{array}{l}0.032(0.027) \\
0.027\end{array}$ & $\begin{array}{l}0.061(0.113) \\
0.027\end{array}$ & $\begin{array}{l}0.004(0.088) \\
0.001\end{array}$ \\
\hline
\end{tabular}

Note: $\mathrm{p}<.05^{*},<.001 * * *$. ALC=alcohol consumption; BMI=body mass index; PI=physical impairment 
Table 51: (Continued)

\begin{tabular}{|c|c|c|c|c|}
\hline & Total & White & Black & Hispanic \\
\hline \multicolumn{5}{|c|}{ Indirect effects of SES on physical impairment via BMI \& ALC } \\
\hline Income $\rightarrow \mathrm{BMI}_{\mathrm{t}=2} \rightarrow \mathrm{PI}_{\mathrm{t}=3}$ & $\begin{array}{l}0.00(0.00) \\
0.00\end{array}$ & $\begin{array}{l}0.00(0.001) \\
0.000\end{array}$ & $\begin{array}{l}0.00(0.001) \\
0.00\end{array}$ & $\begin{array}{l}0.00(0.00) \\
0.00\end{array}$ \\
\hline Income $\rightarrow \mathrm{ALC}_{\mathrm{t}=2} \rightarrow \mathrm{PI}_{\mathrm{t}=3}$ & $\begin{array}{l}0.00(0.00) \\
0.00\end{array}$ & $\begin{array}{l}0.00(0.001) \\
0.000\end{array}$ & $\begin{array}{l}0.00(0.001) \\
0.00\end{array}$ & $\begin{array}{l}0.00(0.00) \\
0.00\end{array}$ \\
\hline Wealth $\rightarrow \mathrm{BMI}_{\mathrm{t}=2} \rightarrow \mathrm{PI}_{\mathrm{t}=3}$ & $\begin{array}{l}0.00(0.00) \\
-0.001\end{array}$ & $\begin{array}{l}-0.0001 *(0.00) \\
-0.001\end{array}$ & $\begin{array}{l}0.00(0.00) \\
0.00\end{array}$ & $\begin{array}{l}-0.001(0.001) \\
-0.004\end{array}$ \\
\hline Wealth $\rightarrow \mathrm{ALC}_{\mathrm{t}=2} \rightarrow \mathrm{PI}_{\mathrm{t}=3}$ & $\begin{array}{l}0.00(0.00) \\
0.00\end{array}$ & $\begin{array}{l}0.00(0.00) \\
0.000\end{array}$ & $\begin{array}{l}0.00(0.00) \\
0.00\end{array}$ & $\begin{array}{l}0.00(0.00) \\
0.00\end{array}$ \\
\hline $\mathrm{Job} \rightarrow \mathrm{BMI}_{\mathrm{t}=2} \rightarrow \mathrm{PI}_{\mathrm{t}=3}$ & $\begin{array}{l}0.001(0.002) \\
0.00\end{array}$ & $\begin{array}{l}0.00(0.00) \\
0.00\end{array}$ & $\begin{array}{l}0.00(0.001) \\
0.00\end{array}$ & $\begin{array}{l}-0.005(0.007) \\
-0.002\end{array}$ \\
\hline $\mathrm{Job} \rightarrow \mathrm{ALC}_{\mathrm{t}=2} \rightarrow \mathrm{PI}_{\mathrm{t}=3}$ & $\begin{array}{l}-0.001(0.001) \\
0.00\end{array}$ & $\begin{array}{l}-0.001(0.001) \\
-0.001\end{array}$ & $\begin{array}{l}-0.003(0.006) \\
-0.001\end{array}$ & $\begin{array}{l}0.00(0.00) \\
0.00\end{array}$ \\
\hline Education $\rightarrow \mathrm{BMI}_{\mathrm{t}=2} \rightarrow \mathrm{PI}_{\mathrm{t}=3}$ & $\begin{array}{l}0.00(0.00) \\
0.00\end{array}$ & $\begin{array}{l}0.00(0.00) \\
0.00\end{array}$ & $\begin{array}{l}0.00(0.00) \\
0.00\end{array}$ & $\begin{array}{l}0.001(0.001) \\
0.003\end{array}$ \\
\hline Education $\rightarrow \mathrm{ALC}_{\mathrm{t}=2} \rightarrow \mathrm{PI}_{\mathrm{t}=3}$ & $\begin{array}{l}0.00(0.00) \\
0.001\end{array}$ & $\begin{array}{l}0.00(0.00) \\
0.001\end{array}$ & $\begin{array}{l}0.001(0.001) \\
0.001\end{array}$ & $\begin{array}{l}0.00(0.00) \\
0.00\end{array}$ \\
\hline
\end{tabular}

Note: $\mathrm{p}<.05^{*},<.001^{* * *}$. ALC=alcohol consumption; BMI=body mass index; PI=physical impairment 
Table 52: Direct and indirect path coefficients for the social integration mediator - lagged path model for overnight hospitalization

Unstandardized coefficients (standard errors) and standardized coefficients.

\begin{tabular}{|c|c|c|c|c|}
\hline & Total & White & Black & Hispanic \\
\hline \multicolumn{5}{|c|}{ Direct effects of SES on overnight hospitalization } \\
\hline Income $\rightarrow \mathrm{OH}_{\mathrm{t}=3}$ & $\begin{array}{l}-0.034(0.026) \\
-0.037\end{array}$ & $\begin{array}{l}-0.03(0.029) \\
-0.03\end{array}$ & $\begin{array}{l}-0.026(0.044) \\
-0.033\end{array}$ & $\begin{array}{l}-0.141 *(0.06) \\
-0.205\end{array}$ \\
\hline Wealth $\rightarrow \mathrm{OH}_{\mathrm{t}=3}$ & $\begin{array}{l}-0.001(0.005) \\
-0.003\end{array}$ & $\begin{array}{l}0.02(0.007) \\
0.007\end{array}$ & $\begin{array}{l}-0.008(0.012) \\
-0.04\end{array}$ & $\begin{array}{l}-0.002(0.016) \\
-0.01\end{array}$ \\
\hline $\mathrm{Job} \rightarrow \mathrm{OH}_{\mathrm{t}=3}$ & $\begin{array}{l}-0.113(0.058)^{*} \\
-0.048\end{array}$ & $\begin{array}{l}-0.128(0.063) \\
-0.054\end{array}$ & $\begin{array}{l}-0.141(0.122) \\
-0.056\end{array}$ & $\begin{array}{l}0.267(0.19) \\
0.101\end{array}$ \\
\hline Education $\rightarrow \mathrm{OH}_{\mathrm{t}=3}$ & $\begin{array}{l}-0.003(0.008) \\
-0.008\end{array}$ & $\begin{array}{l}-0.007(0.011) \\
-0.016\end{array}$ & $\begin{array}{l}0.017(0.018) \\
0.044\end{array}$ & $\begin{array}{l}-0.031(0.018) \\
-0.111\end{array}$ \\
\hline \multicolumn{5}{|c|}{ Direct effects of SES on social integration } \\
\hline Income $\rightarrow \mathrm{SI}_{\mathrm{t}=2}$ & $\begin{array}{l}0.045 * * *(0.009) \\
0.047\end{array}$ & $\begin{array}{l}0.052 * * *(0.013) \\
0.048\end{array}$ & $\begin{array}{l}0.048 * ?(0.027) \\
0.057\end{array}$ & $\begin{array}{l}0.005(0.017) \\
0.007\end{array}$ \\
\hline Wealth $\rightarrow \mathrm{SI}_{\mathrm{t}=2}$ & $\begin{array}{l}0.015 * * *(0.003) \\
0.053\end{array}$ & $\begin{array}{l}0.011 *(0.005) \\
0.035\end{array}$ & $\begin{array}{l}0.018 *(0.005) \\
0.084\end{array}$ & $\begin{array}{l}0.017 *(0.008) \\
0.08\end{array}$ \\
\hline $\mathrm{Job} \rightarrow \mathrm{SI}_{\mathrm{t}=2}$ & $\begin{array}{l}-0.059 * ?(0.031) \\
-0.023\end{array}$ & $\begin{array}{l}-0.059(0.034) \\
-0.024\end{array}$ & $\begin{array}{l}-0.037(0.088) \\
-0.014\end{array}$ & $\begin{array}{l}-0.085(0.097) \\
-0.032\end{array}$ \\
\hline Education $\rightarrow \mathrm{SI}_{\mathrm{t}=2}$ & $\begin{array}{l}0.014 *(0.005) \\
0.034\end{array}$ & $\begin{array}{l}0.012(0.006) \\
0.026\end{array}$ & $\begin{array}{l}-0.003(0.018) \\
-0.007\end{array}$ & $\begin{array}{l}0.01(0.012) \\
0.038\end{array}$ \\
\hline \multicolumn{5}{|c|}{ Effects of social integration on overnight hospitalization } \\
\hline $\mathrm{SI}_{\mathrm{t}=2} \rightarrow \mathrm{OH}_{\mathrm{t}=3}$ & $\begin{array}{l}0.016(0.019) \\
0.021\end{array}$ & $\begin{array}{l}0.025(0.023) \\
0.026\end{array}$ & $\begin{array}{l}-0.052(0.058) \\
-0.055\end{array}$ & $\begin{array}{l}0.11(0.07) \\
0.109\end{array}$ \\
\hline \multicolumn{5}{|c|}{ Indirect effects of SES on overnight hospitalization via social integration } \\
\hline Income $\rightarrow \mathrm{SI}_{\mathrm{t}=2} \rightarrow \mathrm{OH}_{\mathrm{t}=3}$ & $\begin{array}{l}0.001(0.001) \\
0.001\end{array}$ & $\begin{array}{l}0.001(0.001) \\
0.001\end{array}$ & $\begin{array}{l}-0.003(0.003) \\
-0.003\end{array}$ & $\begin{array}{l}0.00(0.002) \\
0.001\end{array}$ \\
\hline Wealth $\rightarrow \mathrm{SI}_{\mathrm{t}=2} \rightarrow \mathrm{OH}_{\mathrm{t}=3}$ & $\begin{array}{l}0.000(0.00) \\
0.001\end{array}$ & $\begin{array}{l}0.000(0.000) \\
0.001\end{array}$ & $\begin{array}{l}-0.001(0.001) \\
-0.005\end{array}$ & $\begin{array}{l}0.002(0.001) \\
0.009\end{array}$ \\
\hline $\mathrm{Job} \rightarrow \mathrm{SI}_{\mathrm{t}=2} \rightarrow \mathrm{OH}_{\mathrm{t}=3}$ & $\begin{array}{l}-0.001(0.001) \\
0.000\end{array}$ & $\begin{array}{l}-0.001(0.001) \\
-0.001\end{array}$ & $\begin{array}{l}0.002(0.005) \\
0.001\end{array}$ & $\begin{array}{l}-0.009(0.011) \\
-0.004\end{array}$ \\
\hline Education $\rightarrow \mathrm{SI}_{\mathrm{t}=2} \rightarrow \mathrm{OH}_{\mathrm{t}=3}$ & $\begin{array}{l}0.000(0.00) \\
0.001\end{array}$ & $\begin{array}{l}0.000(0.00) \\
0.001\end{array}$ & $\begin{array}{l}0.00(0.001) \\
0.000\end{array}$ & $\begin{array}{l}0.001(0.002) \\
0.004\end{array}$ \\
\hline
\end{tabular}

Note: $\mathrm{p}<.05 *,<.001 * * * ; \mathrm{SI}=$ social integration; $\mathrm{OH}=$ overnight hospitalization 
Table 53: Direct and indirect path coefficients for the depressive symptoms mediator-lagged path model for overnight hospitalization

Unstandardized coefficients (standard errors) and standardized coefficients.

\begin{tabular}{|c|c|c|c|c|}
\hline & Total & White & Black & Hispanic \\
\hline \multicolumn{5}{|c|}{ Direct effects of SES on overnight hospitalization } \\
\hline Income $\rightarrow \mathrm{OH}_{\mathrm{t}=3}$ & $\begin{array}{l}-0.032(0.026) \\
-0.035\end{array}$ & $\begin{array}{l}-0.03(0.029) \\
-0.029\end{array}$ & $\begin{array}{l}-0.029(0.044) \\
-0.037\end{array}$ & $\begin{array}{l}-0.486(0.332) \\
-0.627\end{array}$ \\
\hline Wealth $\rightarrow \mathrm{OH}_{\mathrm{t}=3}$ & $\begin{array}{l}0.002(0.005) \\
-0.001\end{array}$ & $\begin{array}{l}0.002(0.007) \\
0.006\end{array}$ & $\begin{array}{l}-0.009(0.012) \\
-0.044\end{array}$ & $\begin{array}{l}-0.013(0.03) \\
-0.53\end{array}$ \\
\hline $\mathrm{Job} \rightarrow \mathrm{OH}_{\mathrm{t}=3}$ & $\begin{array}{l}-0.115 *(0.058) \\
-0.048\end{array}$ & $\begin{array}{l}-0.133 *(0.063) \\
-0.056\end{array}$ & $\begin{array}{l}-0.123(0.116) \\
-0.049\end{array}$ & $\begin{array}{l}5.792(4.943) \\
1.027\end{array}$ \\
\hline Education $\rightarrow \mathrm{OH}_{\mathrm{t}=3}$ & $\begin{array}{l}-0.003(0.007) \\
-0.008\end{array}$ & $\begin{array}{l}-0.007(0.011) \\
-0.017\end{array}$ & $\begin{array}{l}0.019(0.018) \\
0.05\end{array}$ & $\begin{array}{l}-0.075(0.064) \\
-0.241\end{array}$ \\
\hline \multicolumn{5}{|c|}{ Direct effects of SES on depressive symptoms } \\
\hline Income $\rightarrow \mathrm{DS}_{\mathrm{t}=2}$ & $\begin{array}{l}-0.004 *(0.001) \\
-0.035\end{array}$ & $\begin{array}{l}-0.004 *(0.001) \\
-0.03\end{array}$ & $\begin{array}{l}-0.001(0.004) \\
-0.009\end{array}$ & $\begin{array}{l}0.004(0.03) \\
0.036\end{array}$ \\
\hline Wealth $\rightarrow \mathrm{DS}_{\mathrm{t}=2}$ & $\begin{array}{l}-0.003 * * *(0.000) \\
-0.088\end{array}$ & $\begin{array}{l}-0.004 * * *(0.000) \\
-0.099\end{array}$ & $\begin{array}{l}-0.001(0.001) \\
-0.041\end{array}$ & $\begin{array}{l}-0.004 *(0.002) \\
-0.115\end{array}$ \\
\hline Job $\rightarrow \mathrm{DS}_{\mathrm{t}=2}$ & $\begin{array}{l}-0.007(0.005) \\
-0.023\end{array}$ & $\begin{array}{l}-0.006(0.006) \\
-0.022\end{array}$ & $\begin{array}{l}-0.015(0.01) \\
-0.05\end{array}$ & $\begin{array}{l}-0.124(0.531) \\
-0.164\end{array}$ \\
\hline Education $\rightarrow \mathrm{DS}_{\mathrm{t}=2}$ & $\begin{array}{l}-0.001(0.001) \\
-0.025\end{array}$ & $\begin{array}{l}-0.001(0.001) \\
-0.017\end{array}$ & $\begin{array}{l}-0.002(0.002) \\
-0.048\end{array}$ & $\begin{array}{l}0.000(0.004) \\
0.003\end{array}$ \\
\hline \multicolumn{5}{|c|}{ Effects of depressive symptoms on overnight hospitalization } \\
\hline $\mathrm{DS}_{\mathrm{t}=2} \rightarrow \mathrm{OH}_{\mathrm{t}=3}$ & $\begin{array}{l}-0.098(0.186) \\
-0.012\end{array}$ & $\begin{array}{l}-0.194(0.26) \\
-0.023\end{array}$ & $\begin{array}{l}0.371(0.495) \\
0.044\end{array}$ & $\begin{array}{l}1.301(1.758) \\
0.174\end{array}$ \\
\hline \multicolumn{5}{|c|}{ Indirect effects of SES on overnight hospitalization via depressive symptoms } \\
\hline Income $\rightarrow \mathrm{DS}_{\mathrm{t}=2} \rightarrow \mathrm{OH}_{\mathrm{t}=3}$ & $\begin{array}{l}0.00(0.001) \\
0.00\end{array}$ & $\begin{array}{l}0.001(0.001) \\
0.001\end{array}$ & $\begin{array}{l}0.000(0.001) \\
0.000\end{array}$ & $\begin{array}{l}0.005 \\
0.006\end{array}$ \\
\hline Wealth $\rightarrow \mathrm{DS}_{\mathrm{t}=2} \rightarrow \mathrm{OH}_{\mathrm{t}=3}$ & $\begin{array}{l}0.00(0.001) \\
0.00\end{array}$ & $\begin{array}{l}0.001(0.001) \\
0.002\end{array}$ & $\begin{array}{l}-0.000(0.001) \\
-0.002\end{array}$ & $\begin{array}{l}-0.005(0.006) \\
-0.02\end{array}$ \\
\hline $\mathrm{Job} \rightarrow \mathrm{DS}_{\mathrm{t}=2} \rightarrow \mathrm{OH}_{\mathrm{t}=3}$ & $\begin{array}{l}0.00(0.001) \\
0.001\end{array}$ & $\begin{array}{l}0.001(0.002) \\
0.000\end{array}$ & $\begin{array}{l}-0.006(0.007) \\
-0.002\end{array}$ & $\begin{array}{l}-0.161(0.889) \\
-0.029\end{array}$ \\
\hline Education $\rightarrow \mathrm{DS}_{\mathrm{t}=2} \rightarrow \mathrm{OH}_{\mathrm{t}=3}$ & $\begin{array}{l}0.001(0.001) \\
0.00\end{array}$ & $\begin{array}{l}0.000(0.000) \\
0.000\end{array}$ & $\begin{array}{l}-0.001(0.001) \\
-0.002\end{array}$ & $\begin{array}{l}0.000(0.006) \\
0.001\end{array}$ \\
\hline
\end{tabular}

Note: $\mathrm{p} *<0.05, * * *<0.001 . \mathrm{DS}=$ depressive symptoms; $\mathrm{OH}=$ overnight hospitalization 
Table 54: Direct and indirect path coefficients for the behavioral factors mediators-lagged path model for overnight hospitalization

Unstandardized coefficients (standard errors) and standardized coefficients.

\begin{tabular}{|c|c|c|c|c|}
\hline & Total & White & Black & Hispanic \\
\hline \multicolumn{5}{|c|}{ Direct effects of SES on overnight hospitalization } \\
\hline Income $\rightarrow \mathrm{OH}_{\mathrm{t}=3}$ & $\begin{array}{l}-0.032(0.026) \\
-0.035\end{array}$ & $\begin{array}{l}-0.027(0.029) \\
-0.027\end{array}$ & $\begin{array}{l}-0.036(0.045) \\
-0.044\end{array}$ & $\begin{array}{l}-0.142 *(0.06) \\
-0.207\end{array}$ \\
\hline Wealth $\rightarrow \mathrm{OH}_{\mathrm{t}=3}$ & $\begin{array}{l}0.001(0.006) \\
0.002\end{array}$ & $\begin{array}{l}0.003(0.007) \\
0.011\end{array}$ & $\begin{array}{l}-0.01(0.012) \\
-0.05\end{array}$ & $\begin{array}{l}0.001(0.017) \\
0.004\end{array}$ \\
\hline $\mathrm{Job} \rightarrow \mathrm{OH}_{\mathrm{t}=3}$ & $\begin{array}{l}-0.118 *(0.058) \\
-0.049\end{array}$ & $\begin{array}{l}-0.134 *(0.063) \\
-0.056\end{array}$ & $\begin{array}{l}-0.138(0.122) \\
-0.054\end{array}$ & $\begin{array}{l}0.276(0.188) \\
0.104\end{array}$ \\
\hline Education $\rightarrow \mathrm{OH}_{\mathrm{t}=3}$ & $\begin{array}{l}-0.002(0.008) \\
-0.004\end{array}$ & $\begin{array}{l}-0.006(0.011) \\
-0.014\end{array}$ & $\begin{array}{l}0.02(0.018) \\
0.05\end{array}$ & $\begin{array}{l}-0.029(0.017) \\
-0.104\end{array}$ \\
\hline \multicolumn{5}{|c|}{ Direct effects of SES on behavioral variables (BMI \& ALC) } \\
\hline Income $\rightarrow \mathrm{BMI}_{\mathrm{t}=2}$ & $\begin{array}{l}-0.024(0.042) \\
-0.006\end{array}$ & $\begin{array}{l}-0.051(0.051) \\
-0.011\end{array}$ & $\begin{array}{l}-0.024(0.079) \\
-0.006\end{array}$ & $\begin{array}{l}0.129(0.079) \\
0.047\end{array}$ \\
\hline Wealth $\rightarrow \mathrm{BMI}_{\mathrm{t}=2}$ & $\begin{array}{l}-0.012(0.007) \\
-0.006\end{array}$ & $\begin{array}{l}-0.027 *(0.01) \\
-0.019\end{array}$ & $\begin{array}{l}0.021(0.016) \\
0.021\end{array}$ & $\begin{array}{l}0.023(0.028) \\
0.025\end{array}$ \\
\hline $\mathrm{Job} \rightarrow \mathrm{BMI}_{\mathrm{t}=2}$ & $\begin{array}{l}0.013(0.080) \\
0.001\end{array}$ & $\begin{array}{l}0.025(0.089) \\
0.002\end{array}$ & $\begin{array}{l}0.175(0.259) \\
0.014\end{array}$ & $\begin{array}{l}-0.209(0.24) \\
-0.02\end{array}$ \\
\hline Education $\rightarrow \mathrm{BMI}_{\mathrm{t}=2}$ & $\begin{array}{l}-0.014(0.013) \\
-0.008\end{array}$ & $\begin{array}{l}-0.032 *(0.016) \\
-0.016\end{array}$ & $\begin{array}{l}0.03(0.048) \\
0.015\end{array}$ & $\begin{array}{l}0.001(0.02) \\
0.001\end{array}$ \\
\hline Income $\rightarrow \mathrm{ALC}_{\mathrm{t}=2}$ & $\begin{array}{l}0.011 *(0.005) \\
0.012\end{array}$ & $\begin{array}{l}0.026 * * *(0.006) \\
0.025\end{array}$ & $\begin{array}{l}0.009(0.011) \\
0.02\end{array}$ & $\begin{array}{l}-0.002(0.012) \\
-0.006\end{array}$ \\
\hline Wealth $\rightarrow \mathrm{ALC}_{\mathrm{t}=2}$ & $\begin{array}{l}0.002(0.001) \\
0.007\end{array}$ & $\begin{array}{l}0.004 *(0.002) \\
0.012\end{array}$ & $\begin{array}{l}0.000(0.004) \\
-0.001\end{array}$ & $\begin{array}{l}-0.007 *(0.003) \\
-0.059\end{array}$ \\
\hline $\mathrm{Job} \rightarrow \mathrm{ALC}_{\mathrm{t}=2}$ & $\begin{array}{l}0.018(0.17) \\
0.008\end{array}$ & $\begin{array}{l}0.05(0.033) \\
0.021\end{array}$ & $\begin{array}{l}0.008(0.04) \\
0.006\end{array}$ & $\begin{array}{l}0.08(0.042) \\
0.054\end{array}$ \\
\hline Education $\rightarrow \mathrm{ALC}_{\mathrm{t}=2}$ & $\begin{array}{l}0.001(0.002) \\
0.003\end{array}$ & $\begin{array}{l}-0.004(0.003) \\
-0.009\end{array}$ & $\begin{array}{l}0.008 *(0.004) \\
0.038\end{array}$ & $\begin{array}{l}0.000(0.004) \\
-0.002\end{array}$ \\
\hline
\end{tabular}

Note: $*<.05, * * *<.001 ; \mathrm{BMI}=$ body mass index; ALC=alcoholic beverage consumption; $\mathrm{OH}=$ overnight hospitalization 
Table 54: (Continued)

\begin{tabular}{|c|c|c|c|c|}
\hline & Total & White & Black & Hispanic \\
\hline \multicolumn{5}{|c|}{ Effects of behavioral variables (BMI \& ALC) on overnight hospitalization } \\
\hline $\mathrm{BMI}_{\mathrm{t}=2} \rightarrow \mathrm{OH}_{\mathrm{t}=3}$ & $\begin{array}{l}0.015 *(0.005) \\
0.070\end{array}$ & $\begin{array}{l}0.014 *(0.006) \\
0.064\end{array}$ & $\begin{array}{l}0.028 *(0.009) \\
0.142\end{array}$ & $\begin{array}{l}0.01(0.018) \\
0.041\end{array}$ \\
\hline $\mathrm{ALC}_{\mathrm{t}=2} \rightarrow \mathrm{OH}_{\mathrm{t}=3}$ & $\begin{array}{l}0.068 * * *(0.016) \\
0.065\end{array}$ & $\begin{array}{l}0.076 * * *(0.018) \\
0.078\end{array}$ & $\begin{array}{l}-0.215(0.134) \\
-0.115\end{array}$ & $\begin{array}{l}0.013(0.232) \\
0.007\end{array}$ \\
\hline \multicolumn{5}{|c|}{ Indirect effects of SES on overnight hospitalization via behavioral variables (BMI \& ALC) } \\
\hline Income $\rightarrow \mathrm{BMI}_{\mathrm{t}=2} \rightarrow \mathrm{OH}_{\mathrm{t}=3}$ & $\begin{array}{l}0.000(0.001) \\
0.000\end{array}$ & $\begin{array}{l}-0.001(0.001) \\
-0.001\end{array}$ & $\begin{array}{l}-0.001(0.002) \\
-0.001\end{array}$ & $\begin{array}{l}0.001 \\
0.002\end{array}$ \\
\hline Income $\rightarrow \mathrm{ALC}_{\mathrm{t}=2} \rightarrow \mathrm{OH}_{\mathrm{t}=3}$ & $\begin{array}{l}0.001(0.000) \\
0.001\end{array}$ & $\begin{array}{l}0.002 *(0.001) \\
0.002\end{array}$ & $\begin{array}{l}-0.002(0.002) \\
-0.002\end{array}$ & $\begin{array}{l}0.000(0.001) \\
0.000\end{array}$ \\
\hline Wealth $\rightarrow \mathrm{BMI}_{\mathrm{t}=2} \rightarrow \mathrm{OH}_{\mathrm{t}=3}$ & $\begin{array}{l}0.000(0.000) \\
-0.001\end{array}$ & $\begin{array}{l}0.000 *(0.000) \\
-0.001\end{array}$ & $\begin{array}{l}0.001(0.000) \\
0.003\end{array}$ & $\begin{array}{l}0.000(0.00) \\
0.001\end{array}$ \\
\hline Wealth $\rightarrow \mathrm{ALC}_{\mathrm{t}=2} \rightarrow \mathrm{OH}_{\mathrm{t}=3}$ & $\begin{array}{l}0.000(0.000) \\
0.000\end{array}$ & $\begin{array}{l}0.000(0.000) \\
0.001\end{array}$ & $\begin{array}{l}0.000(0.001) \\
0.000\end{array}$ & $\begin{array}{l}0.000(0.002) \\
0.000\end{array}$ \\
\hline $\mathrm{Job} \rightarrow \mathrm{BMI}_{\mathrm{t}=2} \rightarrow \mathrm{OH}_{\mathrm{t}=3}$ & $\begin{array}{l}0.000(0.001) \\
0.000\end{array}$ & $\begin{array}{l}0.000(0.001) \\
0.000\end{array}$ & $\begin{array}{l}0.005(0.008) \\
0.002\end{array}$ & $\begin{array}{l}-0.002(0.005) \\
-0.001\end{array}$ \\
\hline $\mathrm{Job} \rightarrow \mathrm{ALC}_{\mathrm{t}=2} \rightarrow \mathrm{OH}_{\mathrm{t}=3}$ & $\begin{array}{l}0.001(0.001) \\
0.001\end{array}$ & $\begin{array}{l}0.004(0.003) \\
0.002\end{array}$ & $\begin{array}{l}-0.002(0.009) \\
-0.001\end{array}$ & $\begin{array}{l}0.001 \\
0.000\end{array}$ \\
\hline Education $\rightarrow \mathrm{BMI}_{\mathrm{t}=2} \rightarrow \mathrm{OH}_{\mathrm{t}=3}$ & $\begin{array}{l}0.000(0.000) \\
-0.001\end{array}$ & $\begin{array}{l}-0.000(0.000) \\
-0.001\end{array}$ & $\begin{array}{l}0.001(0.001) \\
0.002\end{array}$ & $\begin{array}{l}0.000(0.00) \\
0.000\end{array}$ \\
\hline Education $\rightarrow \mathrm{ALC}_{\mathrm{t}=2} \rightarrow \mathrm{OH}_{\mathrm{t}=3}$ & $\begin{array}{l}0.000(0.000) \\
0.000\end{array}$ & $\begin{array}{l}-0.000(0.000) \\
-0.001\end{array}$ & $\begin{array}{l}-0.002(0.001) \\
-0.004\end{array}$ & $\begin{array}{l}0.000(0.00) \\
0.000\end{array}$ \\
\hline
\end{tabular}

Note: $*<.05, * * *<.001 ; \mathrm{BMI}=$ body mass index; ALC=alcoholic beverage use; $\mathrm{OH}=$ overnight hospitalization 
Table 55: Percent missing data for social, psychological, and behavioral variables over time

\begin{tabular}{|l|l|l|l|l|l|l|}
\hline & \multicolumn{2}{|l}{ White (N=5717) } & \multicolumn{2}{l}{ Black (N=1238) } & \multicolumn{2}{l|}{ Hispanic (N=719) } \\
\hline Variables/Time(T) & Missing & $\%$ & Missing & $\%$ & Missing & $\%$ \\
\hline Social Integration, T=1 & 647 & 11.3 & 156 & 12.6 & 93 & 12.9 \\
\hline Social Integration, T=2 & 932 & 16.3 & 226 & 18.3 & 127 & 17.7 \\
\hline Social Integration, T=3 & 1286 & 22.5 & 364 & 29.4 & 214 & 29.8 \\
\hline Social Integration, T=4 & 1367 & 23.9 & 353 & 28.5 & 197 & 27.4 \\
\hline Social Integration, T=5 & 2006 & 35.1 & 487 & 39.3 & 284 & 39.5 \\
\hline Alcohol consumption, T=1 & 634 & 11.1 & 149 & 12.0 & 93 & 12.9 \\
\hline Alcohol consumption, T=2 & 888 & 15.5 & 213 & 17.2 & 123 & 17.1 \\
\hline Alcohol consumption, T=3 & 1097 & 19.2 & 313 & 25.3 & 181 & 25.2 \\
\hline Body mass index, T=1 & 852 & 14.9 & 227 & 18.3 & 171 & 23.8 \\
\hline Body mass index, T=2 & 1081 & 18.9 & 281 & 22.7 & 195 & 27.1 \\
\hline Body mass index, T=3 & 1285 & 22.5 & 368 & 29.7 & 245 & 34.1 \\
\hline
\end{tabular}


Table 56: Patterns of missing data for social integration over time (+ indicate presence)

\begin{tabular}{|c|llll|}
\hline Race/ethnicity & Pattern & $\begin{array}{l}\text { Missing } \\
\text { values }\end{array}$ & Freq. & $\%$ \\
\hline \multirow{6}{*}{ White } & +++++ & 0 & 3255 & 56.9 \\
&.++++ & 1 & 606 & 10.6 \\
&..+++ & 2 & 274 & 4.8 \\
& $++\ldots$ & 3 & 240 & 4.2 \\
& $+\ldots$. & 4 & 252 & 4.4 \\
&.++++ & 1 & 174 & 3.0 \\
& $\ldots$. & 5 & 449 & 7.9 \\
\hline \multirow{6}{*}{ Black } & +++++ & 0 & 605 & 48.9 \\
&.++++ & 1 & 123 & 9.9 \\
&..+++ & 2 & 73 & 5.9 \\
& $++\ldots$ & 3 & 75 & 6.1 \\
& $+\ldots$. & 4 & 75 & 6.1 \\
&.++++ & 1 & 53 & 4.3 \\
& $\ldots .$. & 5 & 77 & 6.2 \\
\hline \multirow{6}{*}{ Hispanic } & +++++ & 0 & 370 & 51.5 \\
&.++++ & 1 & 78 & 10.8 \\
&..+++ & 2 & 29 & 4.0 \\
&..++ & 3 & 52 & 7.2 \\
& $+\ldots$. & 4 & 26 & 3.6 \\
&.++++ & 1 & 29 & 4.0 \\
& $+\ldots$. & 5 & 66 & 9.2 \\
\hline
\end{tabular}


Table 57: Patterns of missing data for the depressive symptoms factors (psychological factors) (+ indicate presence)

\begin{tabular}{|c|c|c|c|c|c|c|c|c|c|c|c|c|}
\hline \multirow{2}{*}{$\begin{array}{c}\text { Race/ } \\
\text { ethnicity }\end{array}$} & \multicolumn{4}{|c|}{ Negative affect } & \multicolumn{4}{|c|}{ Positive affect } & \multicolumn{4}{|c|}{ Somatic symptoms } \\
\hline & Pattern & $\begin{array}{l}\text { miss } \\
\text { value }\end{array}$ & Freq & $\%$ & Pattern & $\begin{array}{l}\text { miss } \\
\text { value }\end{array}$ & Freq & $\%$ & Pattern & $\begin{array}{l}\text { miss } \\
\text { value }\end{array}$ & Freq & $\%$ \\
\hline \multirow{7}{*}{ White } & +++++ & 0 & 3329 & 58.2 & +++++ & 0 & 3319 & 58.1 & +++++ & 0 & 3228 & 56.5 \\
\hline &.++++ & 1 & 412 & 7.2 & ++++ & 1 & 413 & 7.2 & ++++ . & 1 & 411 & 7.2 \\
\hline &.+++ & 2 & 275 & 4.8 &..+++ & 2 & 272 & 4.8 &.+++ & 2 & 272 & 4.8 \\
\hline & $++\ldots$ & 3 & 213 & 3.7 & $++\ldots$ & 3 & 213 & 3.7 & $++\ldots$ & 3 & 211 & 3.7 \\
\hline & $+\ldots$ & 4 & 229 & 4.0 & $+\ldots$ & 4 & 229 & 4.0 & $+\ldots$ & 4 & 229 & 4.0 \\
\hline &.++++ & 1 & 86 & 1.5 &.++++ & 1 & 81 & 1.4 &.++++ & 1 & 85 & 1.5 \\
\hline & $\ldots \ldots$ & 5 & 661 & 11.6 & $\ldots \ldots$ & 5 & 665 & 11.6 & $\ldots \ldots$ & 5 & 665 & 11.6 \\
\hline \multirow{7}{*}{ Black } & +++++ & 0 & 616 & 49.8 & +++++ & 0 & 613 & 49.5 & +++++ & 0 & 614 & 49.6 \\
\hline &.++++ & 1 & 89 & 7.2 & ++++ & 1 & 93 & 7.5 & ++++ . & 1 & 88 & 7.1 \\
\hline &..+++ & 2 & 75 & 6.1 &..+++ & 2 & 74 & 6.0 &..+++ & 2 & 74 & 6.0 \\
\hline & $++\ldots$ & 3 & 61 & 4.9 & $++\ldots$ & 3 & 61 & 4.9 & $++\ldots$ & 3 & 61 & 4.9 \\
\hline & $+\ldots$ & 4 & 71 & 5.7 & $+\ldots$ & 4 & 74 & 6.0 & $+\ldots$ & 4 & 71 & 5.7 \\
\hline &.++++ & 1 & 24 & 1.9 &.++++ & 1 & 23 & 1.9 &.++++ & 1 & 26 & 2.1 \\
\hline & $\ldots \ldots$ & 5 & 135 & 10.9 & $\ldots \ldots$ & 5 & 134 & 10.8 & $\ldots \ldots$ & 5 & 134 & 10.8 \\
\hline \multirow{7}{*}{ Hispanic } & +++++ & 0 & 349 & 48.5 & +++++ & 0 & 343 & 47.7 & +++++ & 0 & 340 & 47.3 \\
\hline & ++++. & 1 & 53 & 7.4 & ++++ & 1 & 55 & 7.6 & ++++ . & 1 & 58 & 8.1 \\
\hline &..+++ & 2 & 25 & 3.5 &..+++ & 2 & 26 & 3.6 &..+++ & 2 & 25 & 3.5 \\
\hline & $++\ldots$ & 3 & 42 & 5.8 & $++\ldots$ & 3 & 42 & 5.8 & $++\ldots$ & 3 & 42 & 5.8 \\
\hline & $+\ldots$ & 4 & 23 & 3.2 & $+\ldots$ & 4 & 23 & 3.2 & $+\ldots$ & 4 & 23 & 3.2 \\
\hline &.++++ & 1 & 21 & 2.9 &.++++ & 1 & 21 & 2.9 &.++++ & 1 & 20 & 2.8 \\
\hline & $\ldots \ldots$ & 5 & 20 & 2.8 & $\ldots \ldots$ & 5 & 104 & 14.5 & $\ldots \ldots$ & 5 & 104 & 14.5 \\
\hline
\end{tabular}


Table 58: Pattern of missing data for BMI (behavioral factors) over time (+ indicate presence)

\begin{tabular}{|c|c|c|c|c|}
\hline Race/ethnicity & Pattern & Missing values & Freq. & $\%$ \\
\hline \multirow{7}{*}{ White } & +++++ & 0 & 3606 & 63.1 \\
\hline & ++++ & 1 & 389 & 6.8 \\
\hline &..+++ & 2 & 272 & 4.8 \\
\hline & $++\ldots$ & 3 & 230 & 4.0 \\
\hline & $+\ldots$ & 4 & 242 & 4.2 \\
\hline &.++++ & 1 & 63 & 1.1 \\
\hline & $\ldots \ldots$ & 5 & 459 & 8.0 \\
\hline \multirow{7}{*}{ Black } & +++++ & 0 & 686 & 12.0 \\
\hline & ++++. & 1 & 89 & 1.6 \\
\hline &..+++ & 2 & 71 & 1.2 \\
\hline & $++\ldots$ & 3 & 70 & 1.2 \\
\hline & $+\ldots$ & 4 & 65 & 1.1 \\
\hline &.++++ & 1 & 30 & 0.5 \\
\hline & $\ldots \ldots$ & 5 & 82 & 1.4 \\
\hline \multirow{7}{*}{ Hispanic } & +++++ & 0 & 401 & 7.0 \\
\hline & ++++ & 1 & 63 & 1.1 \\
\hline &..+++ & 2 & 31 & 0.5 \\
\hline & $++\ldots$ & 3 & 49 & 0.9 \\
\hline & $+\ldots$ & 4 & 25 & 0.4 \\
\hline &.++++ & 1 & 13 & 0.2 \\
\hline & $\ldots$. & 5 & 67 & 1.2 \\
\hline
\end{tabular}


Table 59: Pattern of missing data for alcohol consumption (behavioral factors) over time (+ indicate presence)

\begin{tabular}{|c|c|c|c|c|}
\hline Race/ethnicity & Pattern & Missing values & Freq. & $\%$ \\
\hline \multirow{7}{*}{ White } & +++++ & 0 & 3692 & 64.6 \\
\hline & ++++ & 1 & 393 & 6.9 \\
\hline &..+++ & 2 & 272 & 4.8 \\
\hline & $++\ldots$ & 3 & 235 & 4.1 \\
\hline & $+\ldots$ & 4 & 242 & 4.2 \\
\hline &.++++ & 1 & 53 & 0.9 \\
\hline & $\ldots \ldots$ & 5 & 443 & 7.7 \\
\hline \multirow{7}{*}{ Black } & +++++ & 0 & 693 & 56.0 \\
\hline & ++++ & 1 & 91 & 7.4 \\
\hline &.+++ & 2 & 72 & 5.8 \\
\hline & $++\ldots$ & 3 & 71 & 5.7 \\
\hline & $+\ldots$ & 4 & 69 & 5.6 \\
\hline &.++++ & 1 & 26 & 2.1 \\
\hline & $\ldots$ & 5 & 75 & 6.1 \\
\hline \multirow{7}{*}{ Hispanic } & +++++ & 0 & 421 & 58.6 \\
\hline & ++++ & 1 & 55 & 7.6 \\
\hline &..+++ & 2 & 32 & 4.5 \\
\hline & $++\ldots$ & 3 & 48 & 6.7 \\
\hline & $+\ldots$ & 4 & 24 & 3.3 \\
\hline &.++++ & 1 & 13 & 1.8 \\
\hline & $\ldots \ldots$ & 5 & 65 & 9.0 \\
\hline
\end{tabular}




\section{Appendix C: Additional RA Results}

\section{Cross-sectional RA models: Fit results}

Table 60: Model IV:JPI:ExPI:SomPI:VoPI for Whites in White vs Hispanic (cross-sectional)

\begin{tabular}{|c|c|c|c|c|c|c|c|c|c|}
\hline & & IV & & Dot & & & Model & & \\
\hline & & IV & & Data & obs. p(DV) & IV) & calc. $\mathrm{q}(\mathrm{DV}$ & IV) & \\
\hline $\mathrm{J}$ & Ex & Som. & $\mathrm{Vo}$ & Freq. & $\mathrm{PI}=0$ & $\mathrm{PI}=1$ & $\mathrm{PI}=0$ & $\mathrm{PI}=1$ & $\mathrm{p}$ (margin) \\
\hline 1 & 0 & 0 & 0 & 33 & 93.939 & 6.061 & 97.46 & 2.54 & 0.027 \\
\hline 1 & 0 & 0 & 1 & 8 & 87.5 & 12.5 & 99.438 & 0.562 & 0.216 \\
\hline 1 & 0 & 1 & 0 & 17 & 100 & 0 & 88.374 & 11.626 & 0.553 \\
\hline 1 & 0 & 1 & 1 & 3 & 100 & 0 & 97.228 & 2.772 & 0.511 \\
\hline 1 & 1 & 0 & 0 & 5 & 100 & 0 & 99.717 & 0.283 & 0.319 \\
\hline 1 & 1 & 0 & 1 & 5 & 100 & 0 & 99.938 & 0.062 & 0.313 \\
\hline 1 & 1 & 1 & 0 & 4 & 100 & 0 & 98.585 & 1.415 & 0.406 \\
\hline 1 & 1 & 1 & 1 & 1 & 100 & 0 & 99.69 & 0.31 & 0.656 \\
\hline 1 & 2 & 0 & 0 & 12 & 100 & 0 & 99.331 & 0.669 & 0.132 \\
\hline 1 & 2 & 0 & 1 & 8 & 100 & 0 & 99.854 & 0.146 & 0.204 \\
\hline 1 & 2 & 1 & 0 & 5 & 80 & 20 & 96.71 & 3.29 & 0.414 \\
\hline 1 & 2 & 1 & 1 & 2 & 100 & 0 & 99.268 & 0.732 & 0.54 \\
\hline 2 & 0 & 0 & 0 & 55 & 87.273 & 12.727 & 90.044 & 9.956 & 0.163 \\
\hline 2 & 0 & 0 & 1 & 15 & 100 & 0 & 97.66 & 2.34 & 0.131 \\
\hline 2 & 0 & 1 & 0 & 79 & 70.886 & 29.114 & 64.177 & 35.823 & 0 \\
\hline 2 & 0 & 1 & 1 & 14 & 92.857 & 7.143 & 89.208 & 10.792 & 0.535 \\
\hline 2 & 1 & 0 & 0 & 14 & 100 & 0 & 98.808 & 1.192 & 0.115 \\
\hline 2 & 1 & 0 & 1 & 5 & 100 & 0 & 99.739 & 0.261 & 0.319 \\
\hline 2 & 1 & 1 & 0 & 12 & 83.333 & 16.667 & 94.261 & 5.739 & 0.298 \\
\hline 2 & 1 & 1 & 1 & 1 & 100 & 0 & 98.698 & 1.302 & 0.675 \\
\hline 2 & 2 & 0 & 0 & 22 & 100 & 0 & 97.22 & 2.78 & 0.076 \\
\hline 2 & 2 & 0 & 1 & 14 & 92.857 & 7.143 & 99.384 & 0.616 & 0.103 \\
\hline 2 & 2 & 1 & 0 & 13 & 84.615 & 15.385 & 87.385 & 12.615 & 0.672 \\
\hline 2 & 2 & 1 & 1 & 2 & 100 & 0 & 96.966 & 3.034 & 0.598 \\
\hline 3 & 0 & 0 & 0 & 26 & 76.923 & 23.077 & 79.139 & 20.861 & 0.603 \\
\hline 3 & 0 & 0 & 1 & 12 & 100 & 0 & 94.596 & 5.404 & 0.284 \\
\hline 3 & 0 & 1 & 0 & 74 & 51.351 & 48.649 & 42.905 & 57.095 & 0 \\
\hline 3 & 0 & 1 & 1 & 10 & 80 & 20 & 77.614 & 22.386 & 0.652 \\
\hline 3 & 1 & 0 & 0 & 6 & 100 & 0 & 97.205 & 2.795 & 0.354 \\
\hline 3 & 1 & 0 & 1 & 6 & 100 & 0 & 99.381 & 0.619 & 0.285 \\
\hline 3 & 1 & 1 & 0 & 4 & 100 & 0 & 87.325 & 12.675 & 0.817 \\
\hline 3 & 1 & 1 & 1 & 2 & 100 & 0 & 96.95 & 3.05 & 0.599 \\
\hline 3 & 2 & 0 & 0 & 9 & 100 & 0 & 93.618 & 6.382 & 0.395 \\
\hline 3 & 2 & 0 & 1 & 4 & 75 & 25 & 98.544 & 1.456 & 0.407 \\
\hline 3 & 2 & 1 & 0 & 7 & 71.429 & 28.571 & 74.396 & 25.604 & 0.546 \\
\hline 3 & 2 & 1 & 1 & 2 & 100 & 0 & 93.059 & 6.941 & 0.704 \\
\hline & & & & 511 & 82.975 & 17.025 & 82.975 & 17.025 & \\
\hline
\end{tabular}




\section{Differences due to non-overlapping predictor variables}

The full model for Whites had only one composite IV state (i.e., J=1, Som $=0, \mathrm{Vo}=0$ and $E x=0$ ) that decreased risk of physical impairment (Appendix $C$, Table 60). This may simply be due to being employed ( $\mathrm{J}=1)$ and absence of somatic symptoms ( $\mathrm{Som}=0$ ) regardless of volunteering and physical activity status. Two sets of composite IV states increased risk and both involved presence of somatic symptoms, absence of physical activity and absence of volunteering in organizations, but these individuals also happened to be retired or in job category 'other'.

Considering the model for Hispanics in totality (Appendix C, Table 61) four composite IV states decreased risk of physical impairment while four other composite IV states increased risk of physical impairment. A key feature was that all four protective composite IV states entailed absence of somatic symptoms and presence of positive affect as well as not falling in job category 'other' $(\mathrm{J}=3)$. Therefore, absence of somatic symptoms and presence of positive affect, regardless of whether one was employed or retired, or had normal weight or was overweight, the risk of physical impairment was low. Three composite IV states that increased risk of physical impairment in Hispanics featured presence of somatic symptoms and absence of positive affect. Overall, being in job category 'other' $(\mathrm{J}=3)$ together with presence of somatic $(\mathrm{Som}=1)$ and absence of positive affect $(\mathrm{Pos}=1)$ increased risk of physical impairment in Hispanics. 
Table 61: Model IV:JPI:BmiPI:SomPI:PosPI for Hispanics in White vs Hispanic

\begin{tabular}{|c|c|c|c|c|c|c|c|c|c|}
\hline \multirow{2}{*}{\multicolumn{4}{|c|}{ IV }} & \multirow{3}{*}{$\begin{array}{l}\text { Data } \\
\text { Freq. }\end{array}$} & \multicolumn{5}{|c|}{ Model } \\
\hline & & & & & \multicolumn{2}{|c|}{ obs. p(DV|IV) } & \multicolumn{2}{|c|}{ calc. $\mathrm{q}(\mathrm{DV} \mid \mathrm{IV})$} & \multirow[b]{2}{*}{$\mathrm{p}($ margin $)$} \\
\hline $\mathrm{J}$ & Bmi. & Pos. & Som. & & $\mathrm{PI}=0$ & $\mathrm{PI}=1$ & $\mathrm{PI}=0$ & $\mathrm{PI}=1$ & \\
\hline 1 & 1 & 0 & 0 & 16 & 100 & 0 & 99.529 & 0.471 & 0.06 \\
\hline 1 & 1 & 0 & 1 & 8 & 75 & 25 & 96.371 & 3.629 & 0.27 \\
\hline 1 & 1 & 1 & 0 & 2 & 100 & 0 & 98.323 & 1.677 & 0.533 \\
\hline 1 & 1 & 1 & 1 & 1 & 100 & 0 & 88.04 & 11.96 & 0.859 \\
\hline 1 & 2 & 0 & 0 & 40 & 100 & 0 & 99.176 & 0.824 & 0.003 \\
\hline 1 & 2 & 0 & 1 & 23 & 95.652 & 4.348 & 93.795 & 6.205 & 0.12 \\
\hline 1 & 2 & 1 & 0 & 1 & 100 & 0 & 97.091 & 2.909 & 0.683 \\
\hline 1 & 2 & 1 & 1 & 4 & 75 & 25 & 80.733 & 19.267 & 0.986 \\
\hline 2 & 1 & 0 & 0 & 24 & 100 & 0 & 98.056 & 1.944 & 0.034 \\
\hline 2 & 1 & 0 & 1 & 10 & 80 & 20 & 86.36 & 13.64 & 0.67 \\
\hline 2 & 1 & 1 & 0 & 4 & 75 & 25 & 93.324 & 6.676 & 0.532 \\
\hline 2 & 1 & 1 & 1 & 11 & 54.545 & 45.455 & 63.704 & 36.296 & 0.141 \\
\hline 2 & 2 & 0 & 0 & 74 & 93.243 & 6.757 & 96.634 & 3.366 & 0.001 \\
\hline 2 & 2 & 0 & 1 & 60 & 75 & 25 & 78.279 & 21.721 & 0.578 \\
\hline 2 & 2 & 1 & 0 & 9 & 88.889 & 11.111 & 88.836 & 11.164 & 0.552 \\
\hline 2 & 2 & 1 & 1 & 20 & 80 & 20 & 49.977 & 50.023 & 0 \\
\hline 3 & 1 & 0 & 0 & 13 & 100 & 0 & 95.038 & 4.962 & 0.199 \\
\hline 3 & 1 & 0 & 1 & 7 & 100 & 0 & 70.627 & 29.373 & 0.513 \\
\hline 3 & 1 & 1 & 0 & 4 & 50 & 50 & 84.15 & 15.85 & 0.876 \\
\hline 3 & 1 & 1 & 1 & 8 & 75 & 25 & 39.996 & 60.004 & 0.003 \\
\hline 3 & 2 & 0 & 0 & 32 & 90.625 & 9.375 & 91.598 & 8.402 & 0.129 \\
\hline 3 & 2 & 0 & 1 & 49 & 57.143 & 42.857 & 57.783 & 42.217 & 0 \\
\hline 3 & 2 & 1 & 0 & 2 & 100 & 0 & 75.137 & 24.863 & 0.83 \\
\hline 3 & 2 & 1 & 1 & 38 & 42.105 & 57.895 & 27.506 & 72.494 & 0 \\
\hline & & & & 460 & 81.087 & 18.913 & 81.087 & 18.913 & \\
\hline
\end{tabular}


Table 62: Model IV:JPI:BmiPI:ExPI:PosPI:SomPI:VoPI for Whites in White vs Black (cross-sectional)

\begin{tabular}{|c|c|c|c|c|c|c|c|c|c|c|c|}
\hline \multirow{2}{*}{\multicolumn{6}{|c|}{ IV }} & \multirow{3}{*}{$\begin{array}{c}\text { Data } \\
\text { Freq. }\end{array}$} & \multicolumn{5}{|c|}{ Model } \\
\hline & & & & & & & \multicolumn{2}{|c|}{ obs. p(DV|IV) } & \multicolumn{3}{|c|}{ calc. $\mathrm{q}(\mathrm{DV} \mid \mathrm{IV})$} \\
\hline $\mathrm{J}$ & Bmi. & Ex & Pos. & Som. & Vo & & $\mathrm{PI}=0$ & $\mathrm{PI}=1$ & $\mathrm{PI}=0$ & $\mathrm{PI}=1$ & $\mathrm{p}($ margin $)$ \\
\hline 1 & 1 & 0 & 0 & 0 & 0 & 15 & 100 & 0 & 96.565 & 3.435 & 0.163 \\
\hline 1 & 1 & 0 & 0 & 0 & 1 & 3 & 100 & 0 & 99.028 & 0.972 & 0.448 \\
\hline 1 & 1 & 0 & 0 & 1 & 0 & 11 & 91.0 & 9.091 & 85.002 & 14.998 & 0.823 \\
\hline 1 & 1 & 0 & 0 & 1 & 1 & 1 & 100 & 0 & 95.358 & 4.642 & 0.732 \\
\hline 1 & 1 & 0 & 1 & 1 & 0 & 5 & 100 & 0 & 61.821 & 38.179 & 0.23 \\
\hline 1 & 1 & 0 & 1 & 1 & 1 & 1 & 100 & 0 & 85.442 & 14.558 & 0.935 \\
\hline 1 & 1 & 1 & 0 & 0 & 0 & 6 & 83.3 & 16.667 & 99.364 & 0.636 & 0.274 \\
\hline 1 & 1 & 1 & 0 & 0 & 1 & 2 & 100 & 0 & 99.824 & 0.176 & 0.516 \\
\hline 1 & 1 & 1 & 0 & 1 & 0 & 1 & 100 & 0 & 96.923 & 3.077 & 0.702 \\
\hline 1 & 1 & 1 & 0 & 1 & 1 & 1 & 100 & 0 & 99.132 & 0.868 & 0.659 \\
\hline 1 & 1 & 2 & 0 & 0 & 0 & 8 & 100 & 0 & 99.511 & 0.489 & 0.203 \\
\hline 1 & 1 & 2 & 0 & 0 & 1 & 6 & 100 & 0 & 99.865 & 0.135 & 0.26 \\
\hline 1 & 1 & 2 & 0 & 1 & 0 & 3 & 100 & 0 & 97.62 & 2.38 & 0.487 \\
\hline 1 & 1 & 2 & 0 & 1 & 1 & 1 & 100 & 0 & 99.332 & 0.668 & 0.655 \\
\hline 1 & 1 & 2 & 1 & 0 & 1 & 1 & 100 & 0 & 99.527 & 0.473 & 0.652 \\
\hline 1 & 1 & 2 & 1 & 1 & 0 & 1 & 100 & 0 & 92.136 & 7.864 & 0.797 \\
\hline 1 & 2 & 0 & 0 & 0 & 0 & 31 & 87.1 & 12.903 & 95.067 & 4.933 & 0.067 \\
\hline 1 & 2 & 0 & 0 & 0 & 1 & 18 & 94.4 & 5.556 & 98.589 & 1.411 & 0.079 \\
\hline 1 & 2 & 0 & 0 & 1 & 0 & 17 & 82.4 & 17.647 & 79.533 & 20.467 & 0.771 \\
\hline 1 & 2 & 0 & 0 & 1 & 1 & 8 & 87.5 & 12.5 & 93.371 & 6.629 & 0.412 \\
\hline 1 & 2 & 0 & 1 & 0 & 0 & 3 & 66.7 & 33.333 & 84.631 & 15.369 & 0.916 \\
\hline 1 & 2 & 0 & 1 & 0 & 1 & 1 & 100 & 0 & 95.229 & 4.771 & 0.735 \\
\hline 1 & 2 & 0 & 1 & 1 & 0 & 7 & 57.1 & 42.857 & 52.612 & 47.388 & 0.057 \\
\hline 1 & 2 & 0 & 1 & 1 & 1 & 2 & 100 & 0 & 80.096 & 19.904 & 0.934 \\
\hline 1 & 2 & 1 & 0 & 0 & 0 & 5 & 100 & 0 & 99.075 & 0.925 & 0.326 \\
\hline 1 & 2 & 1 & 0 & 0 & 1 & 6 & 100 & 0 & 99.743 & 0.257 & 0.263 \\
\hline 1 & 2 & 1 & 0 & 1 & 0 & 2 & 100 & 0 & 95.575 & 4.425 & 0.623 \\
\hline 1 & 2 & 1 & 0 & 1 & 1 & 2 & 100 & 0 & 98.739 & 1.261 & 0.542 \\
\hline 1 & 2 & 1 & 1 & 0 & 0 & 1 & 100 & 0 & 96.836 & 3.164 & 0.703 \\
\hline 1 & 2 & 1 & 1 & 1 & 0 & 1 & 100 & 0 & 86.054 & 13.946 & 0.922 \\
\hline 1 & 2 & 2 & 0 & 0 & 0 & 10 & 100 & 0 & 99.288 & 0.712 & 0.16 \\
\hline 1 & 2 & 2 & 0 & 0 & 1 & 6 & 100 & 0 & 99.803 & 0.197 & 0.262 \\
\hline 1 & 2 & 2 & 0 & 1 & 0 & 4 & 100 & 0 & 96.566 & 3.434 & 0.455 \\
\hline 1 & 2 & 2 & 0 & 1 & 1 & 1 & 100 & 0 & 99.028 & 0.972 & 0.661 \\
\hline 1 & 2 & 2 & 1 & 0 & 0 & 1 & 100 & 0 & 97.552 & 2.448 & 0.689 \\
\hline 1 & 2 & 2 & 1 & 1 & 0 & 1 & 0 & 100 & 88.93 & 11.07 & 0.862 \\
\hline 2 & 1 & 0 & 0 & 0 & 0 & 25 & 92 & 8 & 94.048 & 5.952 & 0.132 \\
\hline 2 & 1 & 0 & 0 & 0 & 1 & 9 & 100 & 0 & 98.284 & 1.716 & 0.209 \\
\hline 2 & 1 & 0 & 0 & 1 & 0 & 33 & 78.788 & 21.212 & 76.11 & 23.89 & 0.358 \\
\hline 2 & 1 & 0 & 0 & 1 & 1 & 6 & 83.333 & 16.667 & 92.03 & 7.97 & 0.532 \\
\hline 2 & 1 & 0 & 1 & 0 & 0 & 4 & 50 & 50 & 81.867 & 18.133 & 0.981 \\
\hline 2 & 1 & 0 & 1 & 0 & 1 & 1 & 100 & 0 & 94.241 & 5.759 & 0.754 \\
\hline 2 & 1 & 0 & 1 & 1 & 0 & 15 & 66.667 & 33.333 & 47.651 & 52.349 & 0.001 \\
\hline 2 & 1 & 0 & 1 & 1 & 1 & 1 & 100 & 0 & 76.74 & 23.26 & 0.884 \\
\hline
\end{tabular}


Table 62: (continued)

\begin{tabular}{|c|c|c|c|c|c|c|c|c|c|c|c|}
\hline \multirow{2}{*}{\multicolumn{6}{|c|}{ IV }} & \multirow{3}{*}{$\begin{array}{l}\text { Data } \\
\text { Freq. }\end{array}$} & \multicolumn{5}{|c|}{ Model } \\
\hline & & & & & & & \multicolumn{2}{|c|}{ obs. p(DV|IV) } & \multicolumn{2}{|c|}{ calc. q(DV|IV) } & \multirow[b]{2}{*}{$\mathrm{p}$ (margin) } \\
\hline $\mathrm{J}$ & Bmi. & Ex. & Pos. & Som. & Vo & & $\mathrm{PI}=0$ & $\mathrm{PI}=1$ & $\mathrm{PI}=0$ & $\mathrm{PI}=1$ & \\
\hline 2 & 1 & 1 & 0 & 0 & 0 & 11 & 100 & 0 & 98.874 & 1.126 & 0.17 \\
\hline 2 & 1 & 1 & 0 & 0 & 1 & 3 & 100 & 0 & 99.687 & 0.313 & 0.43 \\
\hline 2 & 1 & 1 & 0 & 1 & 0 & 3 & 100 & 0 & 94.654 & 5.346 & 0.575 \\
\hline 2 & 1 & 1 & 0 & 1 & 1 & 2 & 100 & 0 & 98.466 & 1.534 & 0.549 \\
\hline 2 & 1 & 1 & 1 & 1 & 0 & 1 & 100 & 0 & 83.496 & 16.504 & 0.975 \\
\hline 2 & 1 & 2 & 0 & 0 & 0 & 12 & 100 & 0 & 99.133 & 0.867 & 0.144 \\
\hline 2 & 1 & 2 & 0 & 0 & 1 & 10 & 100 & 0 & 99.759 & 0.241 & 0.148 \\
\hline 2 & 1 & 2 & 0 & 1 & 0 & 3 & 100 & 0 & 95.842 & 4.158 & 0.539 \\
\hline 2 & 1 & 2 & 0 & 1 & 1 & 3 & 100 & 0 & 98.817 & 1.183 & 0.454 \\
\hline 2 & 1 & 2 & 1 & 1 & 1 & 1 & 100 & 0 & 95.98 & 4.02 & 0.72 \\
\hline 2 & 2 & 0 & 0 & 0 & 0 & 45 & 86.667 & 13.333 & 91.55 & 8.45 & 0.109 \\
\hline 2 & 2 & 0 & 0 & 0 & 1 & 33 & 100 & 0 & 97.517 & 2.483 & 0.024 \\
\hline 2 & 2 & 0 & 0 & 1 & 0 & 69 & 73.913 & 26.087 & 68.597 & 31.403 & 0.003 \\
\hline 2 & 2 & 0 & 0 & 1 & 1 & 20 & 85 & 15 & 88.786 & 11.214 & 0.459 \\
\hline 2 & 2 & 0 & 1 & 0 & 0 & 2 & 50 & 50 & 75.584 & 24.416 & 0.803 \\
\hline 2 & 2 & 0 & 1 & 1 & 0 & 31 & 64.516 & 35.484 & 38.428 & 61.572 & 0 \\
\hline 2 & 2 & 0 & 1 & 1 & 1 & 2 & 100 & 0 & 69.345 & 30.655 & 0.631 \\
\hline 2 & 2 & 1 & 0 & 0 & 0 & 15 & 100 & 0 & 98.367 & 1.633 & 0.116 \\
\hline 2 & 2 & 1 & 0 & 0 & 1 & 19 & 94.737 & 5.263 & 99.544 & 0.456 & 0.055 \\
\hline 2 & 2 & 1 & 0 & 1 & 0 & 4 & 100 & 0 & 92.39 & 7.61 & 0.597 \\
\hline 2 & 2 & 1 & 0 & 1 & 1 & 2 & 100 & 0 & 97.778 & 2.222 & 0.566 \\
\hline 2 & 2 & 1 & 1 & 0 & 1 & 2 & 50 & 50 & 98.422 & 1.578 & 0.55 \\
\hline 2 & 2 & 1 & 1 & 1 & 0 & 2 & 50 & 50 & 77.623 & 22.377 & 0.862 \\
\hline 2 & 2 & 2 & 0 & 0 & 0 & 30 & 96.667 & 3.333 & 98.741 & 1.259 & 0.02 \\
\hline 2 & 2 & 2 & 0 & 0 & 1 & 17 & 94.118 & 5.882 & 99.649 & 0.351 & 0.069 \\
\hline 2 & 2 & 2 & 0 & 1 & 0 & 13 & 100 & 0 & 94.05 & 5.95 & 0.287 \\
\hline 2 & 2 & 2 & 0 & 1 & 1 & 6 & 83.333 & 16.667 & 98.284 & 1.716 & 0.305 \\
\hline 2 & 2 & 2 & 1 & 0 & 0 & 1 & 0 & 100 & 95.726 & 4.274 & 0.725 \\
\hline 2 & 2 & 2 & 1 & 0 & 1 & 2 & 100 & 0 & 98.783 & 1.217 & 0.541 \\
\hline 2 & 2 & 2 & 1 & 1 & 0 & 4 & 75 & 25 & 81.871 & 18.129 & 0.982 \\
\hline 3 & 1 & 0 & 0 & 0 & 0 & 8 & 75 & 25 & 82.665 & 17.335 & 0.979 \\
\hline 3 & 1 & 0 & 0 & 0 & 1 & 4 & 100 & 0 & 94.531 & 5.469 & 0.522 \\
\hline 3 & 1 & 0 & 0 & 1 & 0 & 23 & 60.87 & 39.13 & 49.017 & 50.983 & 0 \\
\hline 3 & 1 & 0 & 0 & 1 & 1 & 3 & 66.667 & 33.333 & 77.702 & 22.298 & 0.834 \\
\hline 3 & 1 & 0 & 1 & 0 & 0 & 4 & 100 & 0 & 57.672 & 42.328 & 0.197 \\
\hline 3 & 1 & 0 & 1 & 1 & 0 & 11 & 36.364 & 63.636 & 21.55 & 78.45 & 0 \\
\hline 3 & 1 & 1 & 0 & 0 & 0 & 3 & 100 & 0 & 96.364 & 3.636 & 0.523 \\
\hline 3 & 1 & 1 & 0 & 0 & 1 & 1 & 100 & 0 & 98.97 & 1.03 & 0.662 \\
\hline 3 & 1 & 1 & 0 & 1 & 0 & 4 & 75 & 25 & 84.236 & 15.764 & 0.92 \\
\hline 3 & 1 & 1 & 0 & 1 & 1 & 1 & 100 & 0 & 95.09 & 4.91 & 0.737 \\
\hline 3 & 1 & 1 & 1 & 1 & 0 & 1 & 100 & 0 & 60.424 & 39.576 & 0.566 \\
\hline
\end{tabular}


Table 62: (continued)

\begin{tabular}{|c|c|c|c|c|c|c|c|c|c|c|c|}
\hline \multirow{2}{*}{\multicolumn{6}{|c|}{ IV }} & \multirow{3}{*}{$\begin{array}{l}\text { Data } \\
\text { Freq. }\end{array}$} & \multicolumn{5}{|c|}{ Model } \\
\hline & & & & & & & \multicolumn{2}{|c|}{ obs. p(DV|IV) } & \multicolumn{2}{|c|}{ calc. q(DV|IV) } & \multirow[b]{2}{*}{$\mathrm{p}($ margin $)$} \\
\hline $\mathrm{J}$ & Bmi. & Ex. & Pos. & Som. & Vo & & $\mathrm{PI}=0$ & $\mathrm{PI}=1$ & $\mathrm{PI}=0$ & $\mathrm{PI}=1$ & \\
\hline 3 & 1 & 2 & 0 & 0 & 0 & 2 & 100 & 0 & 97.184 & 2.816 & 0.581 \\
\hline 3 & 1 & 2 & 0 & 0 & 1 & 1 & 100 & 0 & 99.207 & 0.793 & 0.658 \\
\hline 3 & 1 & 2 & 0 & 1 & 1 & 1 & 100 & 0 & 96.185 & 3.815 & 0.716 \\
\hline 3 & 2 & 0 & 0 & 0 & 0 & 17 & 70.588 & 29.412 & 76.579 & 23.421 & 0.548 \\
\hline 3 & 2 & 0 & 0 & 0 & 1 & 5 & 80 & 20 & 92.218 & 7.782 & 0.561 \\
\hline 3 & 2 & 0 & 0 & 1 & 0 & 48 & 52.083 & 47.917 & 39.731 & 60.269 & 0 \\
\hline 3 & 2 & 0 & 0 & 1 & 1 & 11 & 63.636 & 36.364 & 70.496 & 29.504 & 0.304 \\
\hline 3 & 2 & 0 & 1 & 0 & 0 & 3 & 100 & 0 & 48.299 & 51.701 & 0.123 \\
\hline 3 & 2 & 0 & 1 & 1 & 0 & 26 & 34.615 & 65.385 & 15.85 & 84.15 & 0 \\
\hline 3 & 2 & 0 & 1 & 1 & 1 & 3 & 66.667 & 33.333 & 40.57 & 59.43 & 0.058 \\
\hline 3 & 2 & 1 & 0 & 0 & 0 & 4 & 100 & 0 & 94.784 & 5.216 & 0.513 \\
\hline 3 & 2 & 1 & 0 & 0 & 1 & 2 & 100 & 0 & 98.505 & 1.495 & 0.548 \\
\hline 3 & 2 & 1 & 0 & 1 & 0 & 2 & 50 & 50 & 78.559 & 21.441 & 0.889 \\
\hline 3 & 2 & 1 & 1 & 1 & 0 & 1 & 100 & 0 & 51.144 & 48.856 & 0.414 \\
\hline 3 & 2 & 1 & 1 & 1 & 1 & 2 & 100 & 0 & 79.142 & 20.858 & 0.906 \\
\hline 3 & 2 & 2 & 0 & 0 & 0 & 5 & 100 & 0 & 95.945 & 4.055 & 0.424 \\
\hline 3 & 2 & 2 & 0 & 0 & 1 & 5 & 100 & 0 & 98.847 & 1.153 & 0.333 \\
\hline 3 & 2 & 2 & 0 & 1 & 0 & 4 & 100 & 0 & 82.669 & 17.331 & 0.985 \\
\hline 3 & 2 & 2 & 1 & 1 & 0 & 4 & 75 & 25 & 57.679 & 42.321 & 0.197 \\
\hline 3 & 2 & 2 & 1 & 1 & 1 & 1 & 100 & 0 & 83.164 & 16.836 & 0.982 \\
\hline & & & & & & 865 & 82.312 & 17.688 & 82.312 & 17.688 & \\
\hline
\end{tabular}

\section{Differences due to non-overlapping predictors in full models}

The model for Whites was complex (see Table 13 and 14), with six predicting variables.

Fit results for the full model for Whites thus had sparse distributions (Appendix C, Table 62), where several cells had counts below five. Thus these results should be considered with care. With that caveat in mind, there were seven composite IV states with risks greater than the marginal risk to the sample. Presence of somatic symptoms was a common feature in all seven risky composite IV states. Four out of the seven risky composite IV states involved simultaneous presence of somatic symptoms and absence of positive affect in addition to falling in the retired $(J=2)$ or 'other' $(J=3)$ job categories. Another key feature of the seven sets of risky states was lack of physical activity. Lack of physical activity $(\mathrm{Ex}=0)$, presence of somatic symptoms $(\mathrm{Som}=1)$ and falling in job category 'other' raised the risk in Whites. 
There were only two protective composite IV states in Whites. Both protective IV states entailed being retired $(\mathrm{J}=2)$, and absence of somatic symptoms (Som=0) and presence of positive affect $(\mathrm{Pos}=0)$. The only distinguishing feature of the protective states was that in one protective set, individuals volunteered $\left(\mathrm{Vo}_{\mathrm{O}}=1\right)$ but did not engage in physical activity $(E x=0)$ while in the second protective set, individuals engaged in physical activity but did not volunteer in organizations. It appears, then, that physical activity and volunteering in organizations function in a similar fashion where engaging in these activities was related to absence of physical limitations.

Three composite IV states appeared to reduce risk in the model for Blacks and all involved either being retired $(\mathrm{J}=2)$ or being employed $(\mathrm{J}=1)$ in conjunction with absence of negative affect $(\mathrm{Neg}=0)$ (Appendix $\mathrm{C}$, Table 63). Body mass index did not seem to matter because all these protective states involved falling in the overweight/obese $(\mathrm{Bmi}=2)$ category. An important result that resembles previous findings involved one protective composite IV state that included presence of somatic symptoms (Som=1), where, individuals who were employed and who were overweight but did not have negative affect $(\mathrm{Neg}=0)$ had lower risk than the marginal risk to the sample. There were five sets of risky states, all of which involved being retired or being in job category 'other' in combination with presence of somatic symptoms. Again, body mass index did not seem to matter because risky states involved both normal and overweight categories and negative affect was present in all but one set of risky predictor states. In Blacks, therefore, being retired or falling in job category 'other', together with presence of somatic symptoms and presence of negative affect increased risk, irrespective of BMI status. 
Table 63: Model IV:JPI:BmiPI:SomNegPI for Blacks in White vs Black (cross-sectional)

\begin{tabular}{|c|c|c|c|c|c|c|c|c|c|}
\hline \multirow{2}{*}{\multicolumn{4}{|c|}{ IV }} & \multirow{3}{*}{$\begin{array}{l}\text { Data } \\
\text { Freq. }\end{array}$} & \multicolumn{5}{|c|}{ Model } \\
\hline & & & & & \multicolumn{2}{|c|}{ obs. p(DV|IV) } & \multicolumn{2}{|c|}{ calc. $\mathrm{q}(\mathrm{DV} \mid \mathrm{IV})$} & \multirow[b]{2}{*}{$\mathrm{p}($ margin $)$} \\
\hline $\mathrm{J}$ & Bmi. & Som. & Neg. & & $\mathrm{PI}=0$ & $\mathrm{PI}=1$ & $\mathrm{PI}=0$ & $\mathrm{PI}=1$ & \\
\hline 1 & 1 & 0 & 0 & 15 & 93.333 & 6.667 & 97.972 & 2.028 & 0.094 \\
\hline 1 & 1 & 0 & 1 & 2 & 100 & 0 & 98.75 & 1.25 & 0.509 \\
\hline 1 & 1 & 1 & 0 & 11 & 100 & 0 & 94.273 & 5.727 & 0.262 \\
\hline 1 & 1 & 1 & 1 & 5 & 60 & 40 & 87.7 & 12.3 & 0.67 \\
\hline 1 & 2 & 0 & 0 & 88 & 95.455 & 4.545 & 98.005 & 1.995 & 0 \\
\hline 1 & 2 & 0 & 1 & 17 & 94.118 & 5.882 & 98.77 & 1.23 & 0.054 \\
\hline 1 & 2 & 1 & 0 & 32 & 93.75 & 6.25 & 94.361 & 5.639 & 0.043 \\
\hline 1 & 2 & 1 & 1 & 24 & 100 & 0 & 87.875 & 12.125 & 0.34 \\
\hline 2 & 1 & 0 & 0 & 39 & 87.179 & 12.821 & 92.188 & 7.812 & 0.059 \\
\hline 2 & 1 & 0 & 1 & 10 & 100 & 0 & 95.072 & 4.928 & 0.261 \\
\hline 2 & 1 & 1 & 0 & 31 & 83.871 & 16.129 & 80.083 & 19.917 & 0.999 \\
\hline 2 & 1 & 1 & 1 & 28 & 71.429 & 28.571 & 63.523 & 36.477 & 0.028 \\
\hline 2 & 2 & 0 & 0 & 135 & 93.333 & 6.667 & 92.305 & 7.695 & 0 \\
\hline 2 & 2 & 0 & 1 & 35 & 94.286 & 5.714 & 95.149 & 4.851 & 0.026 \\
\hline 2 & 2 & 1 & 0 & 99 & 76.768 & 23.232 & 80.343 & 19.657 & 0.95 \\
\hline 2 & 2 & 1 & 1 & 90 & 63.333 & 36.667 & 63.902 & 36.098 & 0 \\
\hline 3 & 1 & 0 & 0 & 6 & 83.333 & 16.667 & 80.512 & 19.488 & 0.981 \\
\hline 3 & 1 & 0 & 1 & 1 & 100 & 0 & 87.104 & 12.896 & 0.86 \\
\hline 3 & 1 & 1 & 0 & 14 & 78.571 & 21.429 & 58.466 & 41.534 & 0.051 \\
\hline 3 & 1 & 1 & 1 & 12 & 16.667 & 83.333 & 37.875 & 62.125 & 0 \\
\hline 3 & 2 & 0 & 0 & 33 & 78.788 & 21.212 & 80.768 & 19.232 & 0.923 \\
\hline 3 & 2 & 0 & 1 & 9 & 88.889 & 11.111 & 87.287 & 12.713 & 0.61 \\
\hline 3 & 2 & 1 & 0 & 50 & 64 & 36 & 58.863 & 41.137 & 0 \\
\hline \multirow[t]{2}{*}{3} & 2 & 1 & 1 & 68 & 48.529 & 51.471 & 38.261 & 61.739 & 0 \\
\hline & & & & 854 & 80.094 & 19.906 & 80.094 & 19.906 & \\
\hline
\end{tabular}


Table 64: Model for IV:JPI:BmiPI:SomPI:NegPI for Blacks in Black vs Hispanic (cross-sectional)

\begin{tabular}{|c|c|c|c|c|c|c|c|c|c|}
\hline \multirow{2}{*}{\multicolumn{4}{|c|}{ IV }} & \multirow{3}{*}{$\begin{array}{l}\text { Data } \\
\text { Freq. }\end{array}$} & \multicolumn{5}{|c|}{ Model } \\
\hline & & & & & \multicolumn{2}{|c|}{ obs. p(DV|IV) } & \multicolumn{2}{|c|}{ calc. q(DV|IV) } & \multirow[b]{2}{*}{$p$ (margin) } \\
\hline $\mathrm{J}$ & Bmi. & Som. & Neg. & & $\mathrm{PI}=0$ & $\mathrm{PI}=1$ & $\mathrm{PI}=0$ & $\mathrm{PI}=1$ & \\
\hline 1 & 1 & 0 & 0 & 6 & 83.333 & 16.667 & 98.717 & 1.283 & 0.209 \\
\hline 1 & 1 & 0 & 1 & 1 & 100 & 0 & 96.317 & 3.683 & 0.648 \\
\hline 1 & 1 & 1 & 0 & 5 & 100 & 0 & 94.066 & 5.934 & 0.368 \\
\hline 1 & 1 & 1 & 1 & 4 & 75 & 25 & 84.345 & 15.655 & 0.732 \\
\hline 1 & 2 & 0 & 0 & 47 & 93.617 & 6.383 & 98.616 & 1.384 & 0 \\
\hline 1 & 2 & 0 & 1 & 2 & 100 & 0 & 96.034 & 3.966 & 0.524 \\
\hline 1 & 2 & 1 & 0 & 13 & 92.308 & 7.692 & 93.622 & 6.378 & 0.157 \\
\hline 1 & 2 & 1 & 1 & 15 & 100 & 0 & 83.3 & 16.7 & 0.57 \\
\hline 2 & 1 & 0 & 0 & 23 & 91.304 & 8.696 & 95.438 & 4.562 & 0.037 \\
\hline 2 & 1 & 0 & 1 & 7 & 100 & 0 & 87.669 & 12.331 & 0.507 \\
\hline 2 & 1 & 1 & 0 & 22 & 81.818 & 18.182 & 81.168 & 18.832 & 0.653 \\
\hline 2 & 1 & 1 & 1 & 21 & 66.667 & 33.333 & 59.429 & 40.571 & 0.053 \\
\hline 2 & 2 & 0 & 0 & 57 & 94.737 & 5.263 & 95.091 & 4.909 & 0.001 \\
\hline 2 & 2 & 0 & 1 & 21 & 90.476 & 9.524 & 86.812 & 13.188 & 0.292 \\
\hline 2 & 2 & 1 & 0 & 52 & 76.923 & 23.077 & 79.963 & 20.037 & 0.629 \\
\hline 2 & 2 & 1 & 1 & 54 & 59.259 & 40.741 & 57.559 & 42.441 & 0 \\
\hline 3 & 1 & 0 & 0 & 3 & 100 & 0 & 88.774 & 11.226 & 0.631 \\
\hline 3 & 1 & 0 & 1 & 1 & 100 & 0 & 72.882 & 27.118 & 0.919 \\
\hline 3 & 1 & 1 & 0 & 10 & 70 & 30 & 61.967 & 38.033 & 0.253 \\
\hline 3 & 1 & 1 & 1 & 7 & 14.286 & 85.714 & 35.637 & 64.363 & 0.009 \\
\hline 3 & 2 & 0 & 0 & 18 & 72.222 & 27.778 & 87.984 & 12.016 & 0.274 \\
\hline 3 & 2 & 0 & 1 & 5 & 80 & 20 & 71.333 & 28.667 & 0.757 \\
\hline 3 & 2 & 1 & 0 & 33 & 69.697 & 30.303 & 60.135 & 39.865 & 0.02 \\
\hline 3 & 2 & 1 & 1 & 50 & 48 & 52 & 33.891 & 66.109 & 0 \\
\hline & & & & 477 & 77.149 & 22.851 & 77.149 & 22.851 & \\
\hline
\end{tabular}

\section{Differences due to non-overlapping predictors}

The difference between the Hispanic and Black models was that the Hispanic model had positive affect while the model for Blacks had negative affect as unique predictors. In Blacks, all protective composite IV states involved absence of somatic symptoms, absence of negative affect and not falling in job category 'other' (Appendix C, Table 64). This indicated that employed or retired Blacks, regardless of BMI status, were protected as long as the two psychological variables were absent (i.e., Som $=0 \&$ Neg=0). Four composite IV states increased risk relative to the marginal risk in Blacks. Most of these composite IV states involved falling in job category 'other' and concurrently having somatic symptoms $(\mathrm{Som}=1)$ and negative affect $(\mathrm{Neg}=1)$ irrespective of BMI status. 
Employed $(\mathrm{J}=1)$ or retired $(\mathrm{J}=2)$ Hispanics with positive affect $(\mathrm{Pos}=0)$ and without somatic symptoms (Som=0) were absent, the risk for the individual relative to the sample risk was significantly lower (Appendix C, Table 65). Nearly all (3 out of 4) risky composite IV states in the fit results for Hispanics involved falling in job category 'other', absence of positive affect $(\mathrm{Pos}=0)$ and presence of somatic symptoms $(\mathrm{Som}=1)$ irrespective of BMI status.

Table 65: Model for IV:JPI:BmiPI:SomPI:PosPI for Hispanics in Black vs Hispanic (cross-sectional)

\begin{tabular}{|c|c|c|c|c|c|c|c|c|c|}
\hline \multirow{2}{*}{\multicolumn{4}{|c|}{ IV }} & \multirow{3}{*}{$\begin{array}{c}\text { Data } \\
\text { Freq. }\end{array}$} & \multicolumn{5}{|c|}{ Model } \\
\hline & & & & & \multicolumn{2}{|c|}{ obs. p(DV|IV) } & \multicolumn{2}{|c|}{ calc. q(DV|IV) } & \multirow[b]{2}{*}{$\mathrm{p}($ margin $)$} \\
\hline $\mathrm{J}$ & Bmi. & Pos. & Som. & & $\mathrm{PI}=0$ & $\mathrm{PI}=1$ & $\mathrm{PI}=0$ & $\mathrm{PI}=1$ & \\
\hline 1 & 1 & 0 & 0 & 16 & 100 & 0 & 99.529 & 0.471 & 0.06 \\
\hline 1 & 1 & 0 & 1 & 8 & 75 & 25 & 96.371 & 3.629 & 0.27 \\
\hline 1 & 1 & 1 & 0 & 2 & 100 & 0 & 98.323 & 1.677 & 0.533 \\
\hline 1 & 1 & 1 & 1 & 1 & 100 & 0 & 88.04 & 11.96 & 0.859 \\
\hline 1 & 2 & 0 & 0 & 40 & 100 & 0 & 99.176 & 0.824 & 0.003 \\
\hline 1 & 2 & 0 & 1 & 23 & 95.652 & 4.348 & 93.795 & 6.205 & 0.12 \\
\hline 1 & 2 & 1 & 0 & 1 & 100 & 0 & 97.091 & 2.909 & 0.683 \\
\hline 1 & 2 & 1 & 1 & 4 & 75 & 25 & 80.733 & 19.267 & 0.986 \\
\hline 2 & 1 & 0 & 0 & 24 & 100 & 0 & 98.056 & 1.944 & 0.034 \\
\hline 2 & 1 & 0 & 1 & 10 & 80 & 20 & 86.36 & 13.64 & 0.67 \\
\hline 2 & 1 & 1 & 0 & 4 & 75 & 25 & 93.324 & 6.676 & 0.532 \\
\hline 2 & 1 & 1 & 1 & 11 & 54.545 & 45.455 & 63.704 & 36.296 & 0.141 \\
\hline 2 & 2 & 0 & 0 & 74 & 93.243 & 6.757 & 96.634 & 3.366 & 0.001 \\
\hline 2 & 2 & 0 & 1 & 60 & 75 & 25 & 78.279 & 21.721 & 0.578 \\
\hline 2 & 2 & 1 & 0 & 9 & 88.889 & 11.111 & 88.836 & 11.164 & 0.552 \\
\hline 2 & 2 & 1 & 1 & 20 & 80 & 20 & 49.977 & 50.023 & 0 \\
\hline 3 & 1 & 0 & 0 & 13 & 100 & 0 & 95.038 & 4.962 & 0.199 \\
\hline 3 & 1 & 0 & 1 & 7 & 100 & 0 & 70.627 & 29.373 & 0.513 \\
\hline 3 & 1 & 1 & 0 & 4 & 50 & 50 & 84.15 & 15.85 & 0.876 \\
\hline 3 & 1 & 1 & 1 & 8 & 75 & 25 & 39.996 & 60.004 & 0.003 \\
\hline 3 & 2 & 0 & 0 & 32 & 90.625 & 9.375 & 91.598 & 8.402 & 0.129 \\
\hline 3 & 2 & 0 & 1 & 49 & 57.143 & 42.857 & 57.783 & 42.217 & 0 \\
\hline 3 & 2 & 1 & 0 & 2 & 100 & 0 & 75.137 & 24.863 & 0.83 \\
\hline 3 & 2 & 1 & 1 & 38 & 42.105 & 57.895 & 27.506 & 72.494 & 0 \\
\hline & & & & 460 & 81.087 & 18.913 & 81.087 & 18.913 & \\
\hline
\end{tabular}




\section{Longitudinal RA models: Fit results}

Table 66: Model for IV:ExPI:SomPI:VoPI for Whites in White vs Black (longitudinal)

\begin{tabular}{|c|c|c|c|c|c|c|c|c|}
\hline \multirow{2}{*}{\multicolumn{3}{|c|}{ IV }} & \multirow{3}{*}{$\begin{array}{l}\text { Data } \\
\text { Freq. }\end{array}$} & \multicolumn{5}{|c|}{ Model } \\
\hline & & & & \multicolumn{2}{|c|}{ obs. p(DV|IV) } & \multicolumn{2}{|c|}{ calc. q(DV|IV) } & \multirow[b]{2}{*}{$\mathrm{p}($ margin $)$} \\
\hline Ex. & Som. & Vo & & $\mathrm{PI}=0$ & $\mathrm{PI}=1$ & $\mathrm{PI}=0$ & $\mathrm{PI}=1$ & \\
\hline 0 & 0 & 0 & 151 & 78.808 & 21.192 & 79.852 & 20.148 & 0.968 \\
\hline 0 & 0 & 1 & 62 & 93.548 & 6.452 & 90.159 & 9.841 & 0.043 \\
\hline 0 & 1 & 0 & 175 & 62.857 & 37.143 & 62.303 & 37.697 & 0 \\
\hline 0 & 1 & 1 & 41 & 75.61 & 24.39 & 79.255 & 20.745 & 0.941 \\
\hline 1 & 0 & 0 & 20 & 95 & 5 & 93.922 & 6.078 & 0.114 \\
\hline 1 & 0 & 1 & 24 & 91.667 & 8.333 & 97.277 & 2.723 & 0.032 \\
\hline 1 & 1 & 0 & 27 & 88.889 & 11.111 & 86.566 & 13.434 & 0.376 \\
\hline 1 & 1 & 1 & 8 & 100 & 0 & 93.709 & 6.291 & 0.325 \\
\hline 2 & 0 & 0 & 43 & 93.023 & 6.977 & 91.047 & 8.953 & 0.065 \\
\hline 2 & 0 & 1 & 43 & 95.349 & 4.651 & 95.92 & 4.08 & 0.008 \\
\hline 2 & 1 & 0 & 31 & 77.419 & 22.581 & 80.918 & 19.082 & 0.868 \\
\hline 2 & 1 & 1 & 16 & 93.75 & 6.25 & 90.743 & 9.257 & 0.273 \\
\hline & & & 641 & 79.719 & 20.281 & 79.719 & 20.281 & \\
\hline
\end{tabular}

\section{Differences due to non-overlapping predictors}

The difference between the models was that the unique predictor for Whites was physical activity whereas the model for Blacks had job status and negative affect as unique predictors. In Whites, absence of physical activity $(\mathrm{Ex}=0)$ together with presence of somatic symptoms $(\mathrm{Som}=1)$ and absence of volunteering $\left(\mathrm{Vo}_{\mathrm{o}}=0\right)$ was the only risky set of predictor states. Lack of physical activity and volunteering could be due to respondents' physical impairment status which appears to be closely related to somatic symptoms. Risk of physical impairment in Whites who did not engage in physical activity but volunteered in organizations and were not somatic decreased compared to the marginal risk suggesting that volunteering may work as a substitute for physical activity. The other two protective sets of composite states involved some sort of physical activity and/or volunteering, and absence of somatic symptoms. It is worth emphasizing that all protective states involved volunteering in organizations and absence of somatic symptoms regardless of physical activity status in the full models.

In Blacks, four composite IV states posed significantly lower risk than the marginal risk (Appendix C, Table 67). All protective IV states involved concurrent 
absence of somatic symptoms $(\mathrm{Som}=0)$ and negative affect $(\mathrm{Neg}=0)$, and being employed or retired regardless of the status of volunteering in organizations. But, all risky states involved absence of volunteering in organizations and presence of somatic symptoms in addition to being retired or falling in job category 'other'. Negative affect seemed to matter less because those without and with negative affect were prone to higher risk given the other risky predictor states.

Table 67: Model for IV:JPI:SomPI:NegPI:VoPI for Blacks in White vs Black (longitudinal)

\begin{tabular}{|c|c|c|c|c|c|c|c|c|c|}
\hline \multirow{2}{*}{\multicolumn{4}{|c|}{ IV }} & \multirow{3}{*}{$\begin{array}{l}\text { Data } \\
\text { Freq. }\end{array}$} & \multicolumn{5}{|c|}{ Model } \\
\hline & & & & & \multicolumn{2}{|c|}{ obs. p(DV|IV) } & \multicolumn{2}{|c|}{ calc. q(DV|IV) } & \multirow[b]{2}{*}{$\mathrm{p}($ margin $)$} \\
\hline $\mathrm{J}$ & Som. & Neg. & Vo & & $\mathrm{PI}=0$ & $\mathrm{PI}=1$ & $\mathrm{PI}=0$ & $\mathrm{PI}=1$ & \\
\hline 1 & 0 & 0 & 0 & 32 & 93.75 & 6.25 & 95.392 & 4.608 & 0.033 \\
\hline 1 & 0 & 0 & 1 & 39 & 100 & 0 & 97.629 & 2.371 & 0.007 \\
\hline 1 & 0 & 1 & 0 & 8 & 100 & 0 & 89.2 & 10.8 & 0.534 \\
\hline 1 & 0 & 1 & 1 & 2 & 100 & 0 & 94.263 & 5.737 & 0.623 \\
\hline 1 & 1 & 0 & 0 & 27 & 92.593 & 7.407 & 88.856 & 11.144 & 0.273 \\
\hline 1 & 1 & 0 & 1 & 16 & 93.75 & 6.25 & 94.07 & 5.93 & 0.171 \\
\hline 1 & 1 & 1 & 0 & 18 & 66.667 & 33.333 & 76.085 & 23.915 & 0.636 \\
\hline 1 & 1 & 1 & 1 & 10 & 80 & 20 & 86.356 & 13.644 & 0.64 \\
\hline 2 & 0 & 0 & 0 & 68 & 95.588 & 4.412 & 90.793 & 9.207 & 0.033 \\
\hline 2 & 0 & 0 & 1 & 65 & 95.385 & 4.615 & 95.15 & 4.85 & 0.003 \\
\hline 2 & 0 & 1 & 0 & 11 & 72.727 & 27.273 & 79.735 & 20.265 & 0.949 \\
\hline 2 & 0 & 1 & 1 & 13 & 76.923 & 23.077 & 88.672 & 11.328 & 0.457 \\
\hline 2 & 1 & 0 & 0 & 71 & 76.056 & 23.944 & 79.161 & 20.839 & 0.777 \\
\hline 2 & 1 & 0 & 1 & 43 & 90.698 & 9.302 & 88.314 & 11.686 & 0.196 \\
\hline 2 & 1 & 1 & 0 & 65 & 58.462 & 41.538 & 60.249 & 39.751 & 0 \\
\hline 2 & 1 & 1 & 1 & 17 & 82.353 & 17.647 & 75.096 & 24.904 & 0.574 \\
\hline 3 & 0 & 0 & 0 & 23 & 73.913 & 26.087 & 82.586 & 17.414 & 0.8 \\
\hline 3 & 0 & 0 & 1 & 11 & 100 & 0 & 90.417 & 9.583 & 0.406 \\
\hline 3 & 0 & 1 & 0 & 3 & 33.333 & 66.667 & 65.424 & 34.576 & 0.51 \\
\hline 3 & 0 & 1 & 1 & 2 & 50 & 50 & 79.011 & 20.989 & 0.958 \\
\hline 3 & 1 & 0 & 0 & 32 & 59.375 & 40.625 & 64.624 & 35.376 & 0.023 \\
\hline 3 & 1 & 0 & 1 & 14 & 71.429 & 28.571 & 78.422 & 21.578 & 0.844 \\
\hline 3 & 1 & 1 & 0 & 43 & 55.814 & 44.186 & 42.16 & 57.84 & 0 \\
\hline 3 & 1 & 1 & 1 & 8 & 50 & 50 & 59.186 & 40.814 & 0.128 \\
\hline & & & & 641 & 80.499 & 19.501 & 80.499 & 19.501 & \\
\hline
\end{tabular}


Table 68: Model IV:BmiPI:SomPI:NegPI:VoPI for Blacks in Black vs Hispanic (longitudinal)

\begin{tabular}{|c|c|c|c|c|c|c|c|c|c|}
\hline \multirow{2}{*}{\multicolumn{4}{|c|}{ IV }} & \multirow{3}{*}{$\begin{array}{c}\text { Data } \\
\text { Freq. }\end{array}$} & \multicolumn{5}{|c|}{ Model } \\
\hline & & & & & \multicolumn{2}{|c|}{ obs. p(DV|IV) } & \multicolumn{2}{|c|}{ calc. q(DV|IV) } & \multirow[b]{2}{*}{$\mathrm{p}($ margin $)$} \\
\hline Bmi. & Som. & Neg. & $\mathrm{Vo}$ & & $\mathrm{PI}=0$ & $\mathrm{PI}=1$ & $\mathrm{PI}=0$ & $\mathrm{PI}=1$ & \\
\hline 1 & 0 & 0 & 0 & 21 & 85.714 & 14.286 & 88.993 & 11.007 & 0.222 \\
\hline 1 & 0 & 0 & 1 & 10 & 90 & 10 & 95.402 & 4.598 & 0.201 \\
\hline 1 & 0 & 1 & 0 & 3 & 33.333 & 66.667 & 78.213 & 21.787 & 0.98 \\
\hline 1 & 1 & 0 & 0 & 19 & 84.211 & 15.789 & 70.965 & 29.035 & 0.498 \\
\hline 1 & 1 & 0 & 1 & 6 & 83.333 & 16.667 & 86.249 & 13.751 & 0.612 \\
\hline 1 & 1 & 1 & 0 & 14 & 50 & 50 & 52.043 & 47.957 & 0.022 \\
\hline 1 & 1 & 1 & 1 & 2 & 100 & 0 & 73.578 & 26.422 & 0.891 \\
\hline 2 & 0 & 0 & 0 & 18 & 94.444 & 5.556 & 94.029 & 5.971 & 0.105 \\
\hline 2 & 0 & 0 & 1 & 19 & 100 & 0 & 97.585 & 2.415 & 0.042 \\
\hline 2 & 0 & 1 & 0 & 4 & 75 & 25 & 87.488 & 12.512 & 0.635 \\
\hline 2 & 0 & 1 & 1 & 2 & 50 & 50 & 94.721 & 5.279 & 0.561 \\
\hline 2 & 1 & 0 & 0 & 22 & 77.273 & 22.727 & 82.64 & 17.36 & 0.58 \\
\hline 2 & 1 & 0 & 1 & 11 & 100 & 0 & 92.433 & 7.567 & 0.261 \\
\hline 2 & 1 & 1 & 0 & 32 & 68.75 & 31.25 & 67.883 & 32.117 & 0.194 \\
\hline 2 & 1 & 1 & 1 & 6 & 100 & 0 & 84.433 & 15.567 & 0.688 \\
\hline 3 & 0 & 0 & 0 & 23 & 95.652 & 4.348 & 86.439 & 13.561 & 0.321 \\
\hline 3 & 0 & 0 & 1 & 22 & 100 & 0 & 94.239 & 5.761 & 0.068 \\
\hline 3 & 0 & 1 & 0 & 6 & 83.333 & 16.667 & 73.891 & 26.109 & 0.827 \\
\hline 3 & 0 & 1 & 1 & 4 & 75 & 25 & 87.897 & 12.103 & 0.622 \\
\hline 3 & 1 & 0 & 0 & 33 & 57.576 & 42.424 & 65.833 & 34.167 & 0.11 \\
\hline 3 & 1 & 0 & 1 & 24 & 75 & 25 & 83.177 & 16.823 & 0.522 \\
\hline 3 & 1 & 1 & 0 & 30 & 50 & 50 & 46.106 & 53.894 & 0 \\
\hline \multirow[t]{2}{*}{3} & 1 & 1 & 1 & 13 & 69.231 & 30.769 & 68.704 & 31.296 & 0.441 \\
\hline & & & & 344 & 77.616 & 22.384 & 77.616 & 22.384 & \\
\hline
\end{tabular}

\section{Differences due to non-overlapping variables}

The full model for Blacks was complex and cell-wise distribution of cases was sparse.

Retired Blacks ( $\mathrm{J}=2)$ with concurrent absence of somatic (Som=0) and negative affect $(\mathrm{Neg}=0)$, but who volunteered in organizations $\left(\mathrm{Vo}_{\mathrm{O}}=1\right)$ had significantly lower risk than the marginal risk to the sample. For overweight Blacks, who simultaneously had somatic symptoms and negative affect, and who did not volunteer in organizations, the risk for physical impairment was more than two times the marginal risk to the sample. Thus, volunteering seemed beneficial for Blacks but was not one of the predictive variables in the Hispanic group. All the variables in the model for the Hispanic group overlapped with the variables in the model for Blacks and have been examined. 